\title{
Illustrated guide to Pliocene foraminifera from Miyakojima, Ryukyu Island Arc, with comments on biostratigraphy
}

\author{
Satoshi Hanagata and Takami Nobuhara
}

\begin{abstract}
This is the first comprehensive, fully illustrated systematic report of both planktonic and benthic foraminifera from the Pliocene Shimajiri Group in Miyakojima Island, southern Ryukyu Island Arc. A total of 47 planktonic species in 18 genera and 267 benthic taxa in 151 genera have been identified in 31 matrix samples covering almost all horizons in the island sections. All species, together with morphological varieties, are illustrated using focus-stacking software by assembling digital camera images taken in optical microscopy. These multi-focused illustrations may show natural color and texture (reflection and transmission), and inner structures, including shape of septum, which are unavailable in scanning electron microscopy. Systematic notes comprise comments on synonyms of ubiquitous/well-established species described from other areas. The present comprehensive report with novel illustrations provides an effective guide for future research of foraminifera in surrounding regions.

Planktonic foraminiferal biostratigraphy in Miyakojima Island has also been reexamined by considering sedimentary environment to explain the absence of some index species. The Early to Late Pliocene age of the Shimajiri Group in Miyakojima Island, correlative with the PI1 Zone (Oura Formation), PI1 to PI2 zones (Onogoshi Formation), PI2 to PI4 zones (Yonahama Formation) and PI5 zone (Minebari Formation), respectively, is confirmed.
\end{abstract}

Satoshi Hanagata. Akita-shi Asahikawa-minamimachi 15-21, Akita 010-0834, Japan.

hanagata@ab.auone-net.jp

Takami Nobuhara. Department of Science Education (Geology), Faculty of Education, Shizuoka University, Oya 836, Suruga-ku, Shizuoka 422-8529, Japan etnobuh@ipc.shizuoka.ac.jp

Keywords: taxonomy; foraminifera; Ryukyu; Pliocene; optical micrographs

\section{INTRODUCTION}

\section{Background of Research}

Foraminifera comprise protist species many of which possess hard shells (tests) that have a high fossilization potential. They are distributed across almost all oceans and marginal seas, from shallow coastal waters to abyssal trenches, with each fauna adapting to specific oceanographic conditions. Ecological data extracted from extant fora-

PE Article Number: 18.1.3A

Copyright: Palaeontological Association January 2015

Submission: 28 November 2013. Acceptance: 7 December 2014

Hanagata, Satoshi and Nobuhara, Takami. 2015. Illustrated guide to Pliocene foraminifera from Miyakojima, Ryukyu Island Arc, with comments on biostratigraphy. Palaeontologia Electronica 18.1.3A: 1-140.

palaeo-electronica.org/content/2015/1016-foraminifera-of-miyakojima 

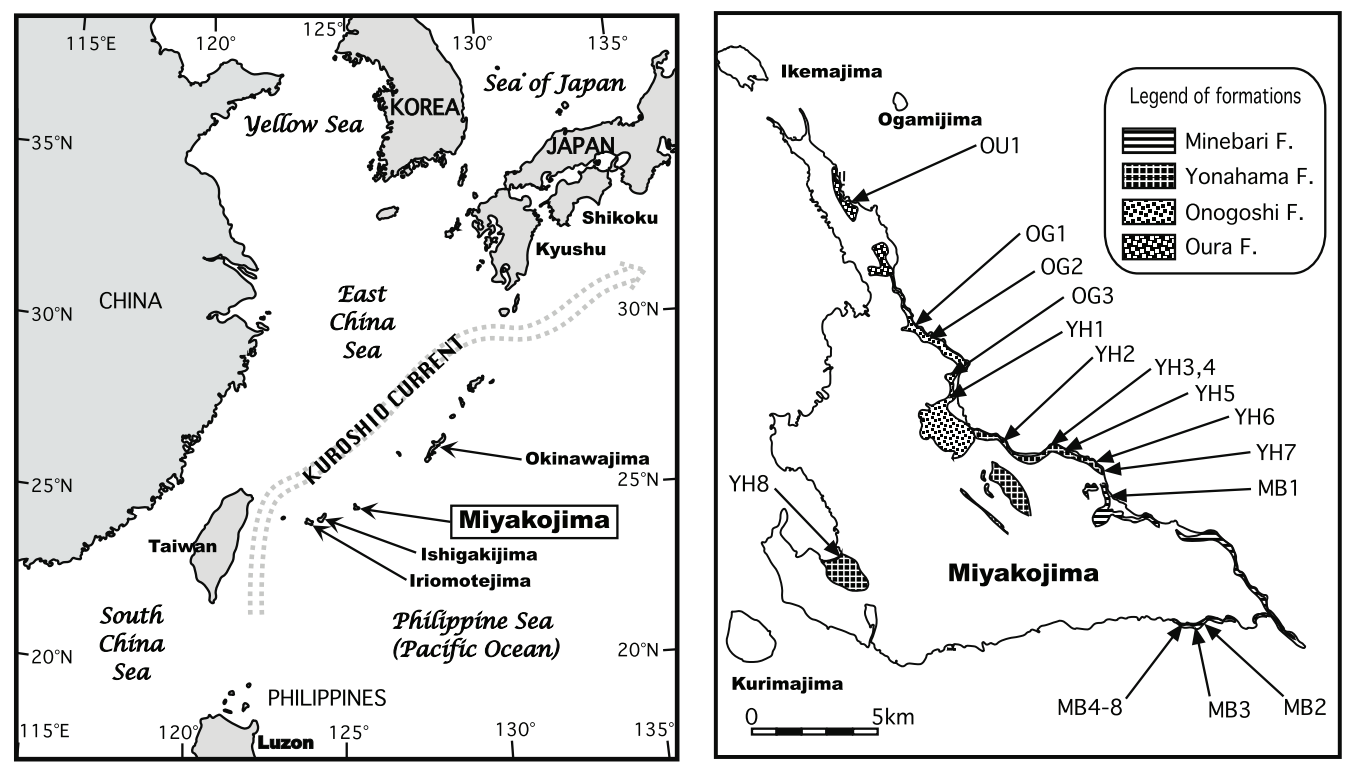

FIGURE 1. Index map of Miyakojima (left) and location of studied fossiliferous outcrops plotted on geologic sketch map compiled after Nakamori (1982) (right).

minifera provide clues for the reconstruction of ancient oceanographic conditions. However, this potential as a paleoenvironmental indicator has not been fully assessed in the Neogene of the subtropical northwestern Pacific region, due to the lack of any descriptive taxonomic studies that are vital to correct identification. Ujiié (1990) and Xu and Ujiié (1994) stressed such problems in foraminiferal taxonomy.

Meanwhile, Scott et al. (2000) pointed out that problems revolve apparently "endemic" species described mainly by Japanese workers from Japanese inland sections and surrounding waters and well-established species described by non-Japanese scientists from other seas, and demonstrated that a number of species could potentially turn out to synonymous or definitely were identical. The Ryukyu Islands are situated between the temperate eastern North Pacific realm and tropical southern seas; thus they are well suited for comparative taxonomic studies.

The Shimajiri Group in Okinawa-jima and Miyakojima islands (Figure 1) records the Neogene history of the Ryukyu Island Arc, and is well-known to yield rich foraminiferal assemblages. In the present report, we focus on foraminiferal fossils of Miyakojima, an island situated in the southwestern Ryukyu Island Arc, at a midpoint between Okinawa-jima and Taiwan.

\section{Review of Previous Reports}

A number of studies have been conducted of both extant and fossil, smaller and larger foraminifera from the Ryukyu Island Arc and surrounding areas in the Philippine Sea (Pacific Ocean) and the East China Sea in terms of oceanography, biostratigraphy and paleontology (e.g., Polski, 1959; LeRoy, 1964; Kameyama, 1975; Ujiié, 1985; Hatta and Ujiié, 1992a, b; Xu and Ujiié, 1994; Ujiié and Hatta, 1995; Hohenegger et al., 1999; Kawagata, 2001). Studies of fossil benthic foraminifera, however, still are few in number in comparison to those of planktonic species in the Ryukyu Islands region, despite the fact that the thick Neogene Shimajiri Group is widely distributed.

From Miyakojima Island, Huang (1968) described fossil foraminifera from three samples of the Shimajiri Group. Later, Ujiié and Ōki (1974) studied planktonic foraminiferal biostratigraphy of the Shimajiri Group in the island, on the basis of 69 matrix samples which covered almost the entire group and correlated it the N17 to N22 zones of Blow (1969). Nakamori (1982) revised this biozonation and equated it with the N18 to N21 zones.

\section{Aim of Present Report}

Based on studies noted above, the present report aims to describe taxonomically both benthic and planktonic foraminifera from conspicuous outcrops in the Pliocene Shimajiri Group in Miyakojima Island, reconfirming the planktonic 
biostratigraphy of previous studies. In particular, we provide focus-stacking illustrations from optical micrographic images of all foraminiferal species, which may be an effective data base for future studies. Not only can such illustrations document outer morphology, but also internal structure in transparent shells, natural color and texture, which are not available in photomicrographs of scanning electron microscope (SEM).

\section{MATERIAL AND METHOD}

\section{Geological Framework}

Miyakojima Island is very flat. The Pleistocene Ryukyu Limestone forms broad terraces across the island; the boundary with the underlying Shimajiri Group is an angular unconformity. The Shimajiri Group is exposed along the east and southeast coast of the island and also around Sugama, the back land of Yonahama Bay, in the southwest of the island (Figure 2; Hanzawa, 1935; Yamazato, 1960; Ujiié and Oki, 1974; Yazaki and Oyama, 1979, 1980; Nakamori, 1982; Furukawa, 1985). In general, the upper sequence appears southeastwardly along exposures of the northeast coast, although exposures are discrete and many minor faults exist between them. The base of the Shimajiri Group is below sea level and inaccessible. Estimates of total thickness of the Shimajiri Group inland vary considerably amongst reports; for instance, Ujiié and Ōki (1974) indicated in excess of $600 \mathrm{~m}$, Yazaki and Oyama (1980) more than $1,000 \mathrm{~m}$, while Nakamori (1982) assumed a thickness of more than $1,400 \mathrm{~m}$.

Doan et al. (1960) and Ujiié and Ōki (1974) described the stratigraphy of the Shimajiri Group in Miyakojima. The former authors subdivided the Shimajiri Group into the Ogami Sandstone, Ikema Sandstone, Gusukube Shale, and Nobaru Formation, in ascending order. However, Ujiié and Ōki (1974) considered the report of Doan et al. (1960) to be a non-public manuscript and alternatively proposed the Nanseien Formation, Yonahama Mudstone, and Minehara Alternation, in ascending order, within the Shimajiri Group. Later, Nakagawa et al. (1976), Yazaki and Okayama (1979, 1980) and successive studies proposed different stratigraphic frames, respectively, without showing sufficient correlation with those proposed in previous studies. Such confusion of stratigraphic nomenclature should be resolved on the basis of the principle of priority; however, this is outside the scope of the present report.
Here we employ Nakamori's (1982) stratigraphic division for convenience in order to compare this with previous faunal studies including those by Ogasawara and Masuda (1983), Sato et al. (2002), and Karasawa and Nobuhara (2008). In ascending order, these units are the Oura, Onogoshi, Yonahama and Minebari formations. The Oura Formation generally corresponds to the Nanseien Formation of Ujiié and Ōki (1974), the Onogoshi and Yonahama formations to the Yonahama Mudstone and the Minebari Formation to the Minehara Alternation, respectively.

The lithology of these formations suggests a single sedimentary cycle: shallow-marine conditions on shelves (sandstone, siltstone, alternating beds of sandstone and siltstone, and conglomerate of the Oura Formation); transgression reaching to the deep sea (massive or partly bedded siltstone of the Onogoshi Formation); further transgression deposited continental slope (massive tuffaceous siltstone of the Yonahama Formation); and basin accumulation (alternating beds of fine sandstone and siltstone of the Minebari Formation). Ogasawara and Masuda (1983) inferred the paleobathymety on the basis of molluscan fossils, to be as follows. The lower part of the Oura Formation was laid down in littoral to inner sublittoral settings. It changed to sandy and muddy sea bottoms of the open sea, shallower than about $50 \mathrm{~m}$ through deposition of the middle and upper parts of the Oura Formation up to the middle of the Onogoshi Formation. The transgression continued and depositional setting turned to pelagic muddy sea bottoms of the lower sublittoral to upper bathyal during deposition of the upper part of the Onogoshi Formation and the Yonahama Formation. Finally, depth reached upper bathyal conditions of around $400 \mathrm{~m}$ in the Minehara (= Minebari) Formation. This bathymetric change was attributed to the rifting stage generated the East China Sea and successive opening of the Okinawa Trough, the active back arc basin of the Ryukyu Island Arc.

The stratigraphical age of each formation has been estimated on the basis of planktonic foraminiferal biostratigraphy in previous studies; this is reviewed below.

\section{Locality and Sampling Horizons}

We have used 30 samples, collected from 19 outcrops along the east and southeast coasts, and a single sample from an outcrop near Sugama, in the southwest of the island. Sampling localities are indicated in Figure 2. Among the 31 samples, 10 indicated by the suffix "-2" were supplementary to 

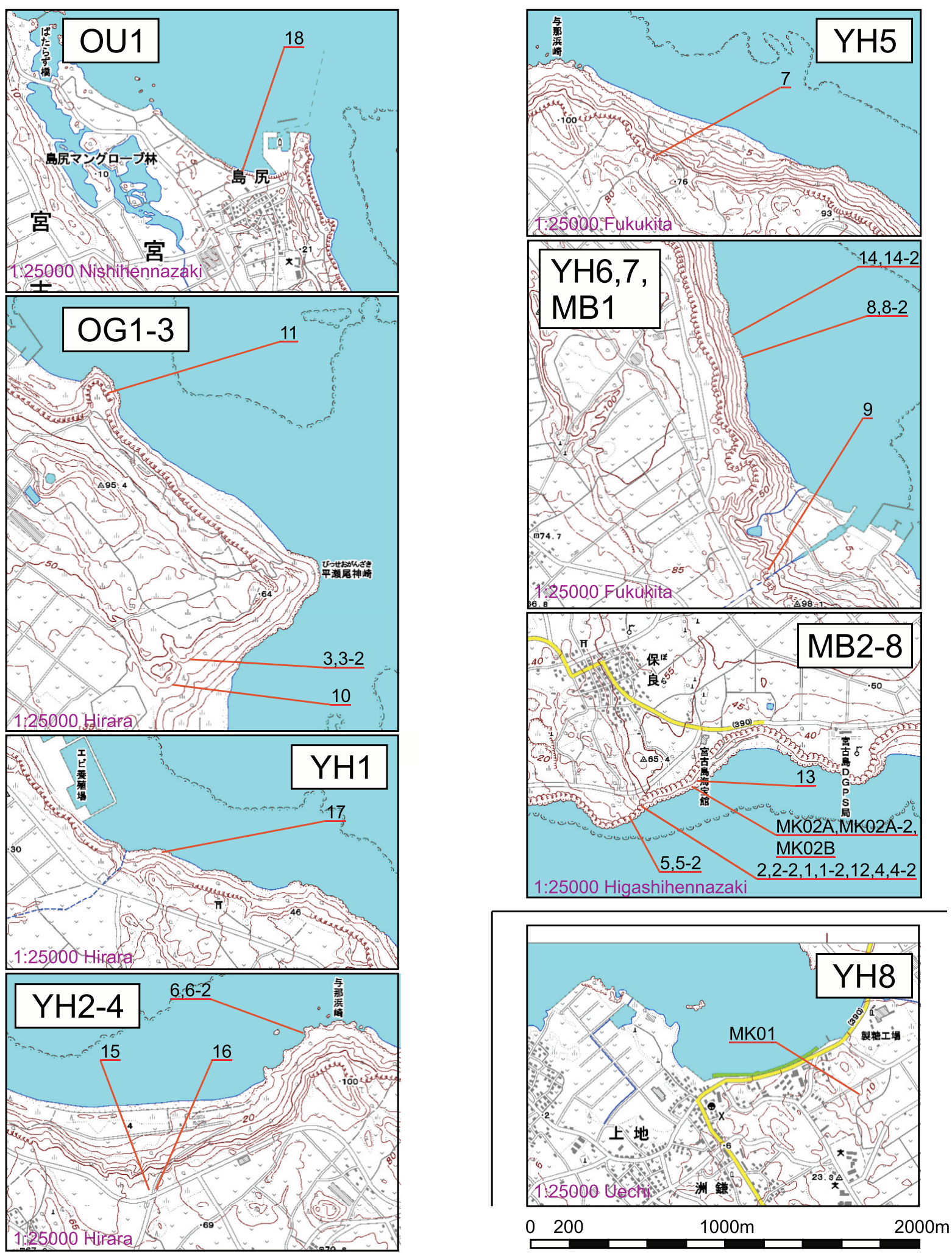

FIGURE 2. Map of sampling sites. Each map is made from 1:25,000 digital map of Geospatial Information Authority of Japan. 
those without this suffix, from adjacent or almost the same horizons so as to confirm the stability of taxonomic composition in close-set positions and/ or to estimate the effect of lithological differences. Stratigraphical relationships between outcrops are difficult to assess in view of concealed faults between outcrops as mentioned by previous workers (e.g., Ujiié and Ōki, 1974; Nakamori, 1982). Therefore, we treat the position of each horizon sampled not by physical but by relative stratigraphical relationship. Details of outcrops are listed in Table 1.

\section{Sample Preparation and Identification}

Rock samples were collected from indurated rocks after excavation of more than $20 \mathrm{~cm}$ from weathered faces in order to obtain fresh material.

All samples, approximately $10 \mathrm{~g}$ each, were dried in an oven for one day. Subsequently, they were soaked in diluted water and heated adding a hydrogen peroxide and sodium pyrophosphate solution, until all matrices had disaggregated. Microscope observation revealed that this procedure did not cause damage to fossil specimens, inclusive of agglutinated species, cemented particles of which retain their original condition. Disintegrated samples were then wet sieved on a 200mesh sieve (75 $\mu$ m-opening). Previous papers on material from Miyakojima Island (see above) used a 120-mesh sieve $(125 \mu \mathrm{m})$ for micropaleontological studies. However, we attach importance to the detection of potentially significant small specimens, so as to obtain the full composition of assemblages. For instance, Alabaminella exigua (Brady) is an important index species of seasonal organic flux in the world ocean (see summary in Murray, 2006), which is usually underestimated in studies using 120-mesh sieve due to its small test size. Meanwhile, we should treat more juvenile specimens that are difficult to identify when using smallsize specimens (e.g., Eguchi et al., 2003; Ujiié, 2003).

Washed residues were split into aliquots, and specimens were picked out until the number of both benthic and planktonic foraminifera exceeded 200. The first author identified all specimens to species, with the exclusion of broken or poorly preserved specimens, so as to illustrate the full composition of the fauna. When no named species was found to assign a particular specimen to, this was listed in open nomenclature as sp. A, sp. B etc., or compared with named species adding either "cf." or "aff."

\section{Illustration}

Here we present pictures of at least two specimens of each species in order to show morphological variation which characterizes most foraminiferal species, whereas almost all previous studies illustrated only typical forms. In addition, we took pictures of each specimen from two or more directions in order to document the threedimensional morphology as an aid in identification.

Specimens are illustrated using an optical microscope with a digital charge-coupled device (ccd) camera and commercial PC software "Helicon Focus" (@Helicon Soft Ltd.). This software creates a single picture from digital images taken at several focus depth slices (Figure 3). The number of pictures used to merge into one picture depends on the size of each specimen, and normally is fewer than 20 , even for the largest specimen. This digital imaging process considerably improves the illustration of smaller-sized foraminifera, which has been advanced thorough the preceding studies including Scott et al. (2000) and Holbourn and Henderson (2002). At present, it is easily operated, and tools are low cost in comparison to SEM images. We depended on SEM to obtain pictures of minute tests under high magnification, but such images do not document the natural appearance that is seen in optical microscopy, inclusive of color, reflection, and transmission. Inner structures of specimens, such as the septum, important for identification, are invisible in SEM as well, but in optical microscopy these are clearly recognized in translucent specimens. This illustration method is also applicable to other taxonomic groups such as radiolarians, ostracodes, and diatoms.

\section{Adoption of Planktonic Foraminiferal Biostratigraphy}

Ujiié and Ōki (1974) reported on the planktonic foraminiferal biostratigraphy of Miyakojima Island. Later, Ujiié (1994) integrated data of planktonic foraminiferal biozonations in the Ryukyu Islands, and proposed a framework. Ujiié's (1994) zones are a combination of Berggren's PI zones (Berggren, 1973, 1977) and Blow's N zones (Blow, 1969). In definition, all of these zones differ, in part, from the recently proposed $\mathrm{PI}$ zones of Berggren et al. (1995). In the present report, we apply the definition of Berggren et al. (1995) in order to avoid confusion among reports. In addition, we mention the revised geological age assignments by Gradstein et al. $(2004,2012)$, and also refer to the biostratigraphical study of the South China Sea by Li et al. (2009). 
TABLE 1. Explanations of outcrop and sample. For detailed sampling locality, see Figure 2.

\begin{tabular}{|c|c|c|c|}
\hline Outcrop & Explanation & Sample & Lithology and sampling position \\
\hline OU1 & $\begin{array}{l}\text { Located in north of Shimajiri settlement northernmost of } \\
\text { Miyakojima; lower three meters, alternations of decimeter } \\
\text { order gray sandy siltstone and fine sandstone beds; upper } \\
\text { three meter, massive siltstone. }\end{array}$ & 18 & $\begin{array}{l}\text { Silty sandstone; taken from uppermost of lower alternation } \\
\text { member. }\end{array}$ \\
\hline OG1 & $\begin{array}{l}\text { Located in cliff at about } 1.2 \mathrm{~km} \text { northwest of Pisseoganzaki } \\
\text { cape; about } 10 \mathrm{~m} \text { high; composed of well-sorted very fine } \\
\text { sandstone or sandy siltstone, contains bivalve shell fossils. }\end{array}$ & 11 & Siltstone; taken from lower part of the outcrop. \\
\hline \multirow[t]{3}{*}{ OG2 } & \multirow{3}{*}{$\begin{array}{l}\text { Located in about } 800 \mathrm{~m} \text { southwest of Pisseoganzaki cape; a } \\
\text { small outcrop of about } 1 \mathrm{~m} \text { high on ground floor of residental } \\
\text { development land; gray clayey siltstone overlain by poorly } \\
\text { sorted sandstone bed containing rip-up clasts; sand-pipes, } \\
1 \mathrm{~cm} \text { in diameter, penetrating into the lower siltstone bed from } \\
\text { the base of upper sandstone bed. }\end{array}$} & 3 & $\begin{array}{l}\text { Mixture of sandstone and siltstone; taken from lower } \\
\text { siltstone bed of exposure but contains sand-pipes } \\
\text { extending from upper sandstone bed. }\end{array}$ \\
\hline & & 3-2-low-silt & Siltstone; taken from the lower bed. \\
\hline & & 3-2-up-sand & Fine sandstone; taken from upper sandstone bed. \\
\hline OG3 & $\begin{array}{l}\text { Located about } 200 \mathrm{~m} \text { southwest of Locality OG2; composed } \\
\text { mainly of massive siltstone and occasionally contains very } \\
\text { fine to medium sandstone. }\end{array}$ & 10 & Siltstone; taken from a massive siltstone part. \\
\hline YH1 & $\begin{array}{l}\text { Located in about } 200 \mathrm{~m} \text { east of shrimp farm south of Takano } \\
\text { fishing port; approximately } 5 \mathrm{~m} \text { high and } 5 \mathrm{~m} \text { wide; composed } \\
\text { of massive and gray siltstone. }\end{array}$ & 17 & Siltstone; taken from middle of the outcrop. \\
\hline \multirow[t]{2}{*}{$\mathrm{YH} 4$} & \multirow{2}{*}{$\begin{array}{l}\text { Located in the western part of Yonahamazaki (cape); } \\
\text { approximately } 5 \mathrm{~m} \text { high; massive siltstone-clayey siltstone } \\
\text { facies. }\end{array}$} & 6 & \multirow{2}{*}{$\begin{array}{l}\text { Both samples } 6 \text { and } 6-2 \text {, siltstone; taken from lowermost of } \\
\text { the exposure, almost the same horizon. }\end{array}$} \\
\hline & & $6-2$ & \\
\hline YH3 & $\begin{array}{l}\text { Located about } 1400 \mathrm{~m} \text { west of Yonahamazaki; about } 2 \mathrm{~m} \text { high; } \\
\text { massive siltstone facies. }\end{array}$ & 16 & Siltstone; taken from upper part of the exposure. \\
\hline $\mathrm{YH} 2$ & $\begin{array}{l}\text { Located close to the } \mathrm{YH} 3 ; 4 \mathrm{~m} \text { high; consists of massive } \\
\text { siltstone with intercalations of thin tuff beds, } 5-10 \mathrm{~cm} \text { rhick. }\end{array}$ & 15 & $\begin{array}{l}\text { Siltstone; taken from approximately } 1 \mathrm{~m} \text { high from the base } \\
\text { of exposure. }\end{array}$ \\
\hline YH5 & $\begin{array}{l}\text { Located about } 700 \mathrm{~m} \text { southeast of Yonahamazaki; about } 15 \mathrm{~m} \\
\text { high; massive siltstone facies, molluscan fossils rare. }\end{array}$ & 7 & Siltstone; taken from basal part of the exposure. \\
\hline \multirow[t]{2}{*}{ YH6 } & \multirow{2}{*}{$\begin{array}{l}\text { Coastal cliff, located } 2300 \mathrm{~m} \text { southeast of Yonahamazaki; } 3 \mathrm{~m} \\
\text { high and } 3 \mathrm{~m} \text { wide ; massive siltstone facies. }\end{array}$} & 14 & \multirow{2}{*}{$\begin{array}{l}\text { Both samples } 14 \text { and } 14-2 \text {, siltstone; taken from the basal } \\
\text { part of the exposure. }\end{array}$} \\
\hline & & $14-2$ & \\
\hline \multirow[t]{2}{*}{$\mathrm{YH} 7$} & \multirow{2}{*}{$\begin{array}{l}\text { Coastal cliff, } 200 \mathrm{~m} \text { south of the YH6; } 5-6 \mathrm{~m} \text { high and } 10 \mathrm{~m} \\
\text { wide; consists mostly of massive siltstone and intercalates } \\
\text { several thin }(2-3 \mathrm{~cm}) \text { fine acidic tuff layers and sandy siltstone } \\
\text { beds. }\end{array}$} & 8 & $\begin{array}{l}\text { Sandy siltstone; taken from } 3 \mathrm{~m} \text { high from base of the } \\
\text { exposure. }\end{array}$ \\
\hline & & $8-2$ & $\begin{array}{l}\text { Siltstone; taken from about } 20 \mathrm{~cm} \text { higher horizon of sample } \\
8 \text {. }\end{array}$ \\
\hline YH8 & $\begin{array}{l}\text { Located near Sugama village, southwestern part of } \\
\text { Miyakojima; about } 1 \mathrm{~m} \text { high; composed of massive siltstone; } \\
\text { probably correlatable to the lower part of the Yonahama } \\
\text { formation in northeast coast section. }\end{array}$ & MK01 & Siltstone; taken from the base of exposure. \\
\hline MB1 & $\begin{array}{l}\text { Located about } 1 \mathrm{~km} \text { south of the } \mathrm{YH} 7,500 \mathrm{~m} \text { inland from the } \\
\text { Urasoko beach: about } 20 \mathrm{~m} \text { high; rhysmic alternations of fine } \\
\text { sandstone and siltstone, sanstone beds } 2-5 \mathrm{~cm} \text { thick and } \\
\text { siltstone beds } 10-20 \mathrm{~cm} \text {; only one outcrop of Minebari } \\
\text { Formation in east coast of Miyakojima in this study. }\end{array}$ & 9 & Siltstone; no molluscan fossil. \\
\hline MB4 & $\begin{array}{l}\text { Located in south coast of the island, south of Bora settlement } \\
\text { (also MB2 8); about } 1 \mathrm{~m} \text { high; massive clayey siltstone rich in } \\
\text { trace fossils; molluscan fossil not found. }\end{array}$ & $5,5-2$ & $\begin{array}{l}\text { Both samples } 5 \text { and } 5-2 \text {, siltstone; taken from almost the } \\
\text { same horizon, middle of the exposure. }\end{array}$ \\
\hline \multirow[t]{2}{*}{ MB5 } & \multirow{2}{*}{$\begin{array}{l}\text { Located about } 10 \mathrm{~m} \text { above the MB } 4 ; 4 \mathrm{~m} \text { high and } 18 \mathrm{~m} \text { width; } \\
\text { bedded alternation of sandy siltstone intercalating thin fine } \\
\text { sandstone. }\end{array}$} & 2 & $\begin{array}{l}\text { Taken from sandy siltstone bed of alternation; diverse } \\
\text { molluscan fossils included. }\end{array}$ \\
\hline & & $2-2$ & $\begin{array}{l}\text { Taken about } 1 \mathrm{~m} \text { below the sample } 2 \text {, close to the bottom of } \\
\text { exposure; lithology is the same as sample } 2 \text {. }\end{array}$ \\
\hline MB6 & $\begin{array}{l}\text { Located about } 7 \mathrm{~m} \text { above the MB5; almost massive facies } \\
\text { intercalating thin sandstone beds; This outcrop and following } \\
\text { MB7 and MB8 expose almost continuously. }\end{array}$ & $1,1-2$ & $\begin{array}{l}\text { Both samples } 1 \text { and } 1-2 \text {, sandy siltstone; taken from } \\
\text { almost the same horizon of massive sandy siltstone facies. }\end{array}$ \\
\hline MB7 & $\begin{array}{l}\text { Located about } 50 \mathrm{~cm} \text { above the MB6; siltstones intercalating } \\
\text { thin sandstone beds. }\end{array}$ & 12 & $\begin{array}{l}\text { siltstone just above thin sandstone bed rich in fossil } \\
\text { foraminifers }\end{array}$ \\
\hline \multirow[t]{2}{*}{ MB8 } & \multirow[t]{2}{*}{ Located above MB7. } & 4 & $\begin{array}{l}\text { Pumiceous massive sandy siltstone; taken about } 0.5 \mathrm{~m} \\
\text { above the horizon of Sample } 12 \text {. }\end{array}$ \\
\hline & & $4-2$ & $\begin{array}{l}\text { Pumiceous massive sandy siltstone; about } 1 \mathrm{~m} \text { above the } \\
\text { Sample } 4 \text {. }\end{array}$ \\
\hline
\end{tabular}


TABLE 1 (continued).

\begin{tabular}{|c|c|c|c|}
\hline Outcrop & Explanation & Sample & Lithology and sampling position \\
\hline \multirow[t]{3}{*}{ MB3 } & Basal part of coastal cliff located in west of Bora beach; $1-2 m$ & MK02A & Sandy siltstone including abundant molluscan fossils. \\
\hline & $\begin{array}{l}\text { thick below a large floatstone of the Ryukyu Limestone; } \\
\text { sandy siltstone and sandstone; a number of sand-pipes in } \\
\text { the silty part. }\end{array}$ & MK02A-2 & $\begin{array}{l}\text { Sandy siltstone; almost the same horizon of Sample } \\
\text { MK02A. }\end{array}$ \\
\hline & & MK02B & $\begin{array}{l}\text { Siltstone; taken from massive siltstone bed above the } \\
\text { mollusca-rich sandstone bed of Sample MK02A. }\end{array}$ \\
\hline MB2 & $\begin{array}{l}\text { Small outcrop of fine silty sandstone contaning abundat rip- } \\
\text { up clasts of siltstone in chatic texture, about } 2 \mathrm{~m} \text { high and } 2 \mathrm{~m} \\
\text { wide below limestone block; significant bioturbation, } \\
\text { including bivalve and fragmental simple coral fossils. }\end{array}$ & 13 & Taken from a sandy siltstone clast of the exposure. \\
\hline
\end{tabular}

\section{RESULTS AND DISCUSSION}

More than 200 specimens of both planktonic and benthic foraminifera were collected from each sample, with the exception of Sample 9 of the Minebari Formation.

Forty-seven planktonic foraminiferal species, belonging to 18 genera, and 267 benthic foraminiferal species, in 151 genera, are distinguished, inclusive of forms recorded in open nomenclature (i.e., sp. A, sp. B, etc.; Table 2 which is available online only). In addition, the first author examined dry-sieved specimens larger than $125 \mu \mathrm{m}$, yielding more than 200 planktonic foraminiferal individuals from the Minebari Formation to confirm the extinction level of Dentoglobigerina altispira (Cushman and Jarvis) and the occurrence of Globorotalia tosaensis (Takayanagi and Saito) and Globorotalia
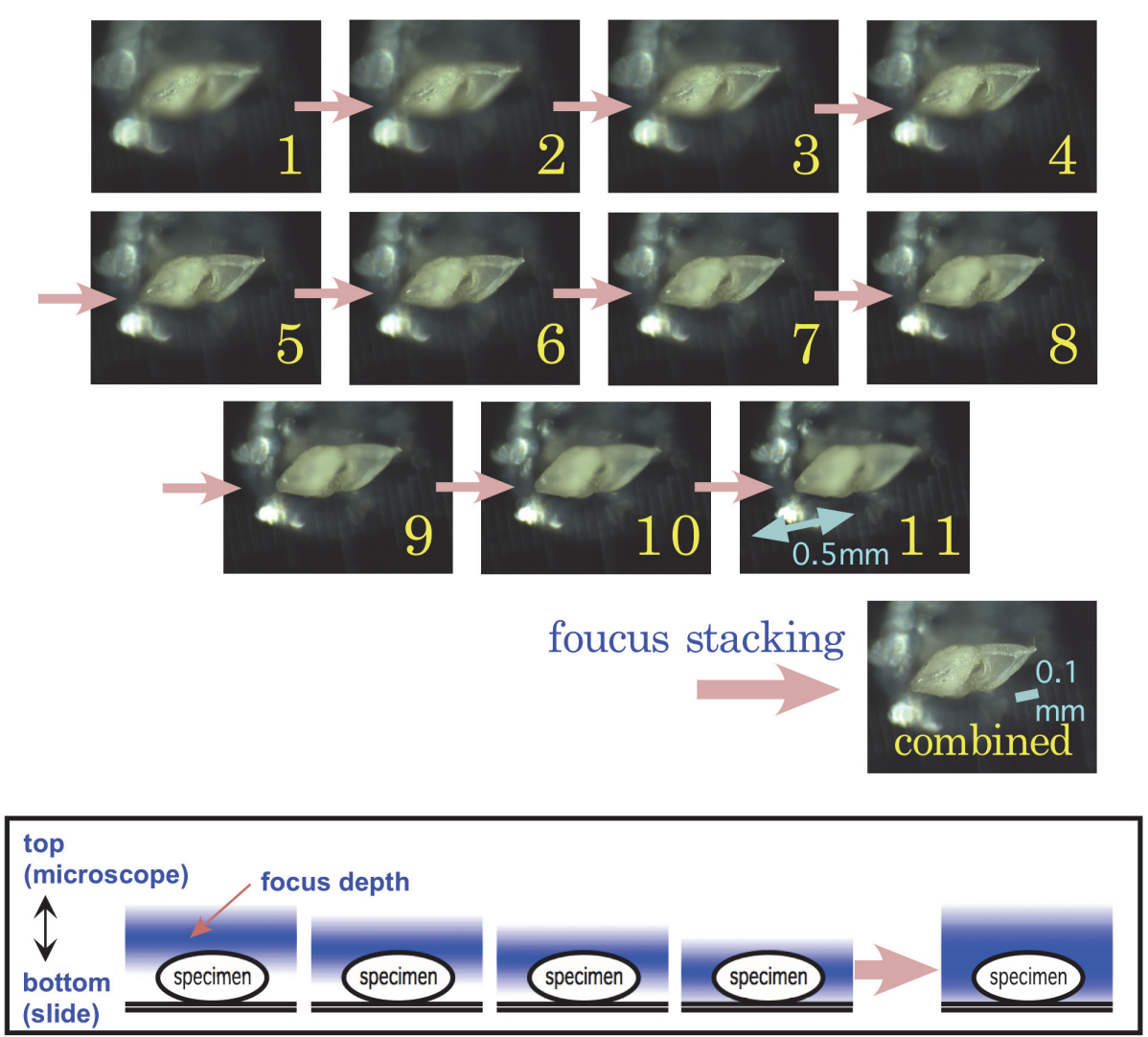

FIGURE 3. Image examples of focus stacking process. Combined image is processed gathering clearly focused part from pictures taken at several sequential focus depths. Above example is edge view of Globorotalia cultrata from sample MK01. The figure below in the frame is a principle image of focus stacking of optical micrograph. Specimen is mounted on a microscale slide to see its size (background line is used to make scale bar). 
truncatulinoides (d'Orbigny). In short, $D$. altispira is absent from the Minebari Formation and neither $G$. tosaensis nor $G$. truncatulinoides have been recognized in the study material.

In general, the preservation of specimens is good, except for those from samples 11, 3, 3-2low-silt, 3-2-up-sand, 10, 2-2, MK02A-2, and MK02B (weak dissolution, probably caused by weathering), and samples 7, MK02A, and 13 (carbonate recrystallization). These specimens of poor preservation are difficult to identify. The effect of dissolution on calcareous specimens called "fragmentation", caused under the lysocline or CCD (e.g., Le and Shackleton, 1992; Xu et al., 2005), was not recognized in microscope observation.

Indeterminate juvenile specimens (in 200mesh sieve) are small in number; however, several diminutive specimens caused identification problems; allied species occasionally show common morphological features at the juvenile stage.

\section{General Features of Foraminiferal Assemblages}

The benthic foraminiferal assemblages show a quite high diversity (evenness) and almost all of the abundant species account for less than 30\% (Figure 4). Exceptions are Euuvigerina vadescens in the Onogoshi Formation (36\% in Sample 11), Bolivina robusta Brady in the Onogoshi Formation (41\% in Sample 10), and Uvigerina peregrina in the Yonahama Formation (46\% in Sample 6-2; $35 \%$ in Sample 6). Planktonic foraminiferal assemblages also show a high diversity.

Oura Formation. The diversity of benthic foraminifera is lower than in overlying formations. Heterolepa dutemplei (d'Orbigny) is the predominant benthic species, accounting for $28 \%$ of the assemblage. Asterorotalia pulchella (d'Orbigny), Ammonia maruhasii (Kuwano), and Hanzawaia nipponica Asano also characterize this benthic fauna. The percentage of agglutinated foraminifera vs total benthic foraminifera is highest in the study material (approximately 18\%). The diversity of planktonic foraminifera is also low in comparison to overlying units. Globigerina foliata Bolli is the commonest planktonic species, accounting for $28 \%$ of assemblages. Globigerinoides ruber (d'Orbigny) and Globigerinoides sacculifers (Brady) are also abundant.

Onogoshi Formation. Sample 11 from the lower part of this unit is characterized by the abundant occurrence of Euuvigerina vadescens in association with Ammonia maruhasii and B. robusta. Samples from the upper part of the formation yield abundant $A$. maruhasii, B. robusta, Bulimina margi- nata d'Orbigny, Cassidulina carinata Silvestri, Cibicidoides pachyderma (Rzehak), Fontbotia wuellerstorfi (Schwager), and Gyroidinoides altiformis (Stewart and Stewart). Globigerina foliata and species of Globigerinoides are abundant in the planktonic foraminiferal assemblages.

Yonahama Formation. The composition of benthic foraminiferal assemblages varies among samples from this unit. Bolivina robusta is abundant in almost all samples. Cibicidoides ornatus (Cushman) is common in the lower horizon (Samples 17, 6, 6-2). Uvigerina peregrina Cushman markedly predominates in Samples 6 and 6-2, accompanied by common Heterolepa praecincta (Karrer). Globocassidulina subglobosa (Brady), Bulimina truncana Gümbel, and Myllostomella hyugaensis (Ishizaki) in the middle part of the formation (Samples 16, 15, 7). Ammonia parkinsoniana (d'Orbigny), and Bulimina gutta Chapman and Parr are relatively abundant in Samples 14 and 14-2. Samples of the uppermost horizon (Samples 8, 8-2) characteristically contain Bulimina aculeata d'Orbigny. Globigerina foliata, Globigerinita glutinata (Egger), and species of Globigerinoides are abundant in planktonic assemblages. Neogloboquadrina blowi (Rögl and Hansen) predominates (24\%) in Sample 6-2, the benthic assemblage of which is characterized by a marked predominance of $U$. peregrina as noted above.

One sample from the Yonahama Formation in Sugama, in the southwest of the island (Sample MK01), yielded relatively abundant Cassidulina carinata, Hyalinea balthica (Schröter), B. robusta, and Bolivina cochei Cushman and Jarvis in benthic assemblages. Globigerina foliata, G. glutinata and Turborotalita quinqueloba (Natland) are common in the planktonic assemblage.

Minebari Formation. Bolivina robusta and Cassidulina carinata constantly and abundantly occur in benthic foraminiferal assemblages of all samples. Although there is not a single species that predominates, several taxa are abundant in certain samples, as follows: Bolivinita quadrilatera (Schwager) in Samples 1, 1-2, and MK02B; Burseolina pacifica (Cushman) in Samples 2 and 2-2; Evolvocassidulina brevis (Aoki) in Sample 5; Heterolepa dutemplei in Samples 5-2 and 4-2; Melonis pacificus (Cushman) in Sample 9; Nonionellina labradorica (Dawson) in Sample MK02B; Uvigerina peregrina in Samples 2 and 4-2. Planktonic foraminiferal assemblages are similar to those of the Yonahama Formation. Species of Globigerinoides, Globoturborotalita rubescens (Hofker), and G. quinqueloba 


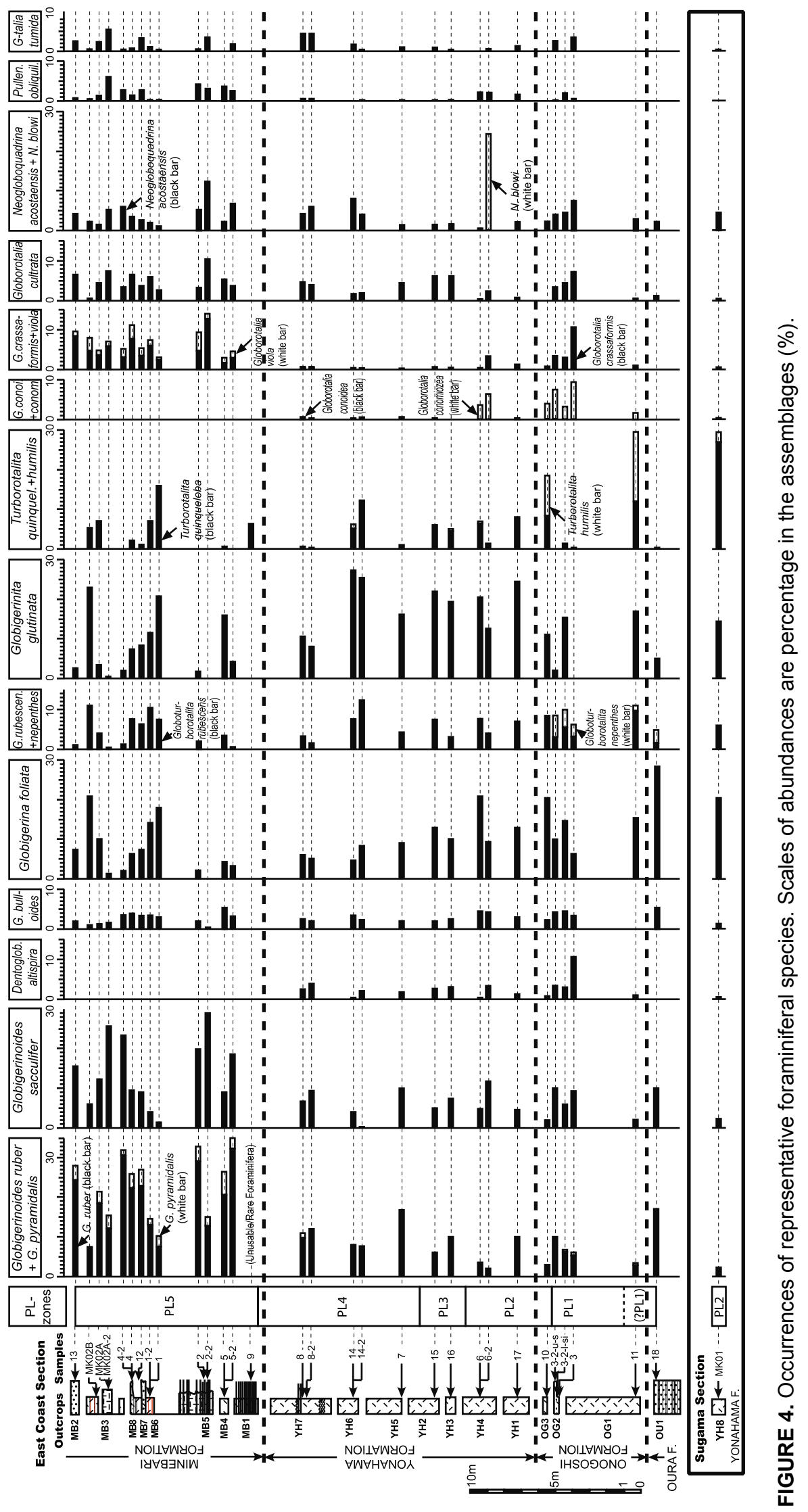




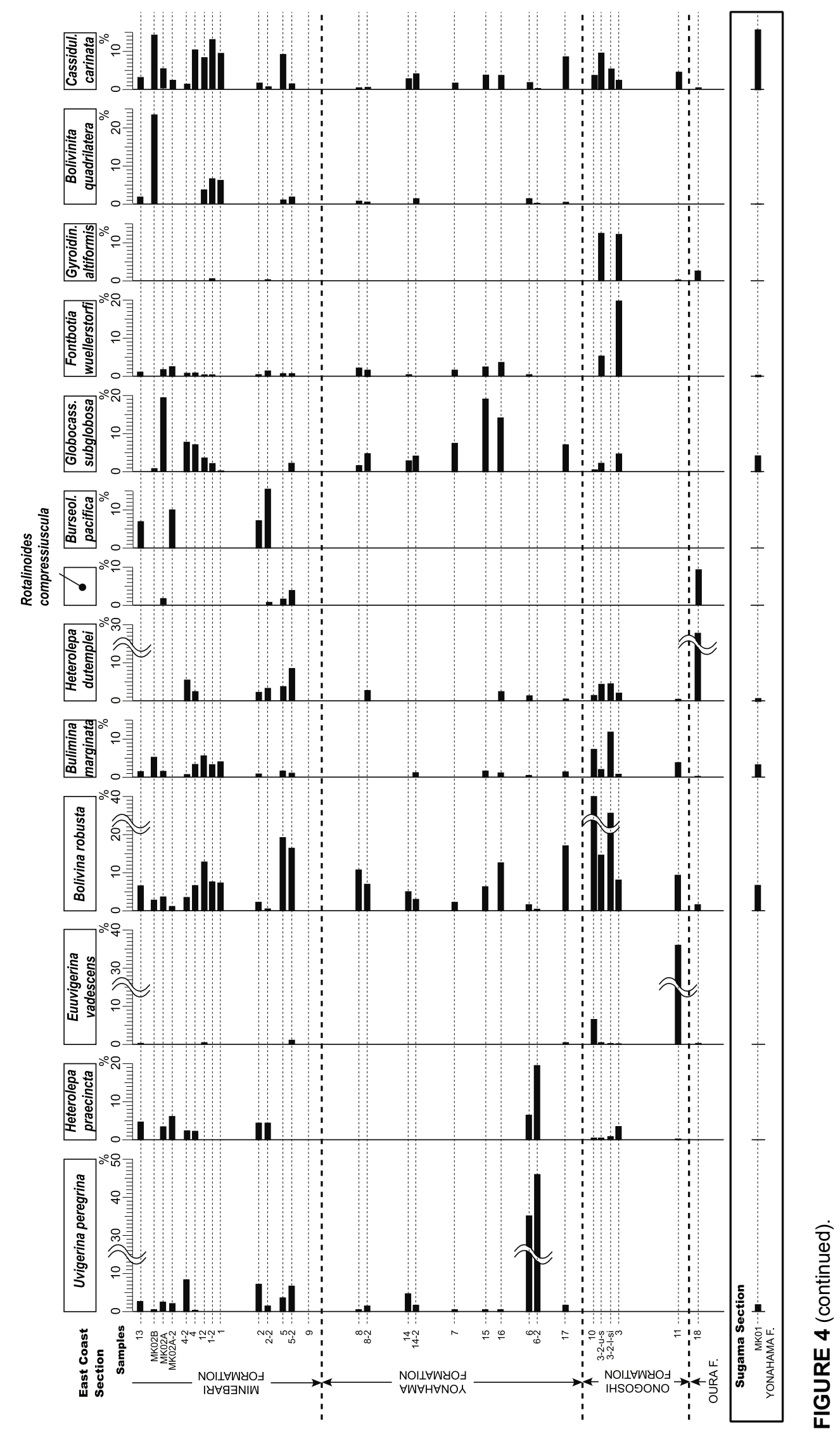


show a higher abundance in comparison with assemblages of the Yonahama Formation.

\section{Planktonic Foraminiferal Biostratigraphy}

The following biohorizons of planktonic foraminifera are recognized in the Shimajiri Group of Miyakojima. Based on these, the group was equated with the planktonic foraminiferal zones PI1 to PI5 (Figure 5). This result is consistent with data in previous studies (Ujiié and Oki, 1974; Nakamori, 1982).

First occurrence of Globorotalia tumida (Brady). This marks the lower limit of PI1 in Sample 3 of the Onogoshi Formation.

Last occurrence of Globoturborotalita nepenthes (Todd). This marks the upper limit of PI1 in Sample 3-2-up-sand of the Onogoshi Formation.

Last occurrence of Globorotalia margaritae Bolli and Bermudez. This marks the upper limit of $\mathrm{PI} 2$ in Sample 6 of the Yonahama Formation.

Last occurrence of Sphaeroidinellopsis spp. This marks the upper limit of $\mathrm{PI} 3$ in Sample 15 of the Yonahama Formation.

Last occurrence of Dentoglobigerina altispira. This marks the upper limit of PI4 in Sample 8 of the Yonahama Formation.

Other biohorizons. The following occurrences of planktonic foraminifera are potentially useful for stratigraphy: the first occurrence of Globorotalia crassaformis in Sample 16 of the Yonahama Formation; the occurrence of Globorotalia conomiozea Kennett between Sample 18 of the Oura Formation and Sample 6 of the Yonahama Formation; the occurrence of Globorotalia conoidea Walters between Samples 18 and 8, both Yonahama Formation; the first occurrence of Sphaeroidinella dehiscens (Parker and Jones) in Sample 7 of the Yonahama Formation, not accompanying Sphaeroidinellopsis seminulina (Schwager); change in coiling direction of Pulleniatina obliquiloculata (Parker and Jones) from sinistral to dextral, twice between Samples 6 and 16, and Samples 7 and 14-2, both in the Yonahama Formation; the last occurrence of Globigerinoides obliquus Bolli in Sample 15 of the Yonahama Formation; the last occurrence of Globigerinoides extremus Bolli and Bermúdez in Sample 9 of the Minebari Formation; the last occurrence of Globoturborotalita decoraperta (Takayanagi) in Sample 4-2 of the Minebari Formation.

Other well-known Plio-Pleistocene datum events such as the last occurrence of Globorotalia pseudomiocenica Bolli and Bermudez, the last occurrence of Globigerinoidesella fistulosa
(Schubert), the first occurrence of $G$. truncatulinoides, and the last occurrence of $G$. tosaensis, have not been recognized.

\section{Geological Age}

The age of the lowermost horizon of the Shimajiri Group in Miyakojima has been controversial. Ujiié and Oki (1974) pointed out the existence of Miocene strata in the lower part of the Shimajiri Group, on the basis of the first occurrence of Globorotalia tumida in the lower part of the Yonahama Mudstone, equivalent to the Onogoshi Formation of the present study. The absence of G. tumida from the lowermost of the group (i.e., Oura Formation and lower part of Onogoshi Formation) is also confirmed in our material. The first occurrence of $G$. tumida (5.57 Ma; Gradstein et al., 2012) marks the base of PI1, which is very close to the age of the Miocene/Pliocene boundary (5.33 Ma; Gradstein et al., 2012). Meanwhile, Nakagawa et al. (1976) refuted the existence of the Miocene in Miyakojima on the basis of a magnetostratigraphical study. Nakamori (1982) also dated the Oura Formation as Pliocene, on the basis of the occurrence of the calcareous nannofossil Ceratoliths cf. rugosus Bukry and Bramlette. If Nakamori's identification is correct (Ceratolithus rugosus being indicative of the base of zone NN13 of Martini, 1971), it is clear that at least the horizon sampled is of Early Pliocene age (5.12 Ma; Gradstein et al., 2004). Considering that the Oura Formation was laid down under sublittoral conditions, as noted by Ogasawara and Masuda (1983), G. tumida which was an inhabitant of the deep thermocline (Nathan and Leckie, 2009) could not have spread. Thus, it would be inappropriate to base the age on the absence of G. tumida; another approach is needed to date the age of the base of the Oura Formation.

The last occurrence of Globoturborotalita nepenthes, i.e., the upper limit of PI1, is in Sample 3-2-up-sand (outcrop OG2, Onogoshi Formation). Berggren et al. (1995) dated this horizon at 4.18 Ma, while Gradstein et al. $(2004,2012)$ showed 4.37 Ma. This horizon is almost the same as that of the first occurrence of Globorotalia tumida in the present study; thus, the absence of G. tumida from the lower horizon is inferred to be an effect of depositional condition as mentioned above, while the disappearance of $G$. nepenthes in the bathyal sediments of the present study is more reliable.

The change in coiling direction of Pulleniatina spp. from sinistral to dextral has been observed twice in the Yonahama Formation. The reason behind this is unknown, but stratigraphic repetition 

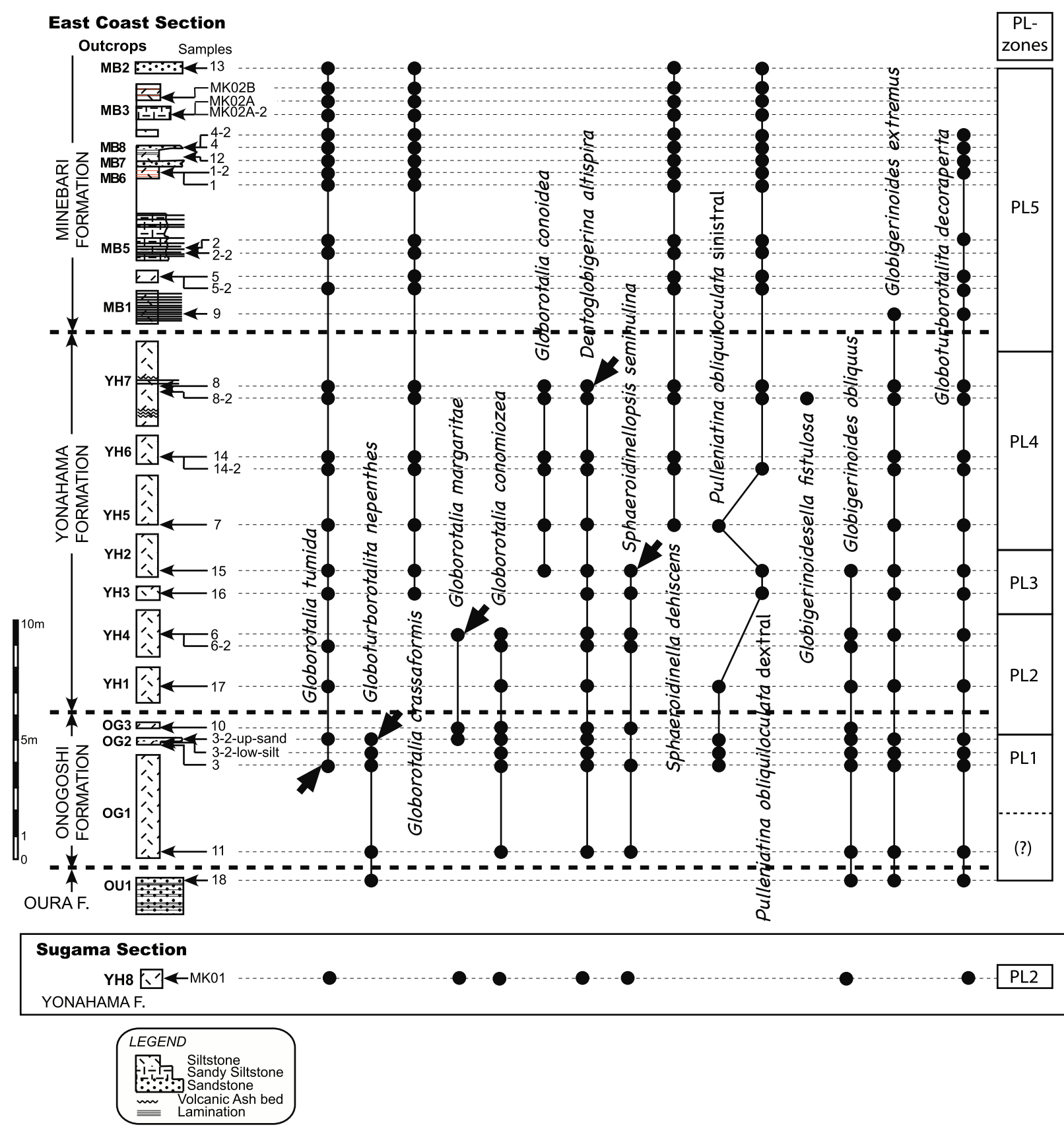

FIGURE 5. Lithologic column of outcrops, occurrences of index and stratigraphically important planktonic foraminifera. Stratigraphic intervals of each outcrop are not to scale. Thick arrows in the figure indicate first or last occurrences of index and stratigraphically important species of planktonic foraminiferal zones.

by faults is the most probable cause. Its age was estimated to be $3.95 \mathrm{Ma}$ by Saito (1999) and 4.08 Ma by Gradstein et al. (2012).

The age of the last occurrence of Globorotalia margaritae, which marks the upper limit of $\mathrm{PI}$, was estimated at $3.58 \mathrm{Ma}$ by Berggren et al. (1995) and at 3.85 Ma by Gradstein et al. (2004, 2012).

The first occurrence of Globorotalia crassaformis (4.31 Ma; Gradstein et al., 2012) corresponds to a level above the above-mentioned last occurrence of Globorotalia margaritae and of the change in coiling of Pulleniatina spp. Test calcification in G. crassaformis is under seasonal thermocline (Ravelo and Fairbanks, 1992), which is why its absence from the lower strata implies an environmental effect similar to that for Globorotalia tumida, and its first occurrence in Miyakojima Island does not conform to the date of its evolutionary appearance. 
Berggren et al. (1995) dated the extinction level of Sphaeroidinellopsis spp., which defines the top of $\mathrm{PI}$, at $3.12 \mathrm{Ma}$; this was accepted in subsequent papers (Saito, 1999; Chaisson and D'Hondt, 2000). Gradstein et al. $(2004,2012)$ preferred 3.59 Ma for the upper limit of the range of Sphaeroidinellopsis seminulina (Schwager). However, Kučera (1998) stressed that the replacement of $S$. seminulina by Sphaeroidinella dehiscens sensu stricto, which is distinguished by the development of a large supplementary aperture, occurred in the Atlantic and Indian oceans and the eastern equatorial Pacific Ocean over a short time span of about 50 kyr between 3.5-3.6 Ma. Kučera (1998) referred to this horizon as the "Sphaeroidinella event." This event approximately predates the top of PI3 by 0.4 myr (Berggren et al., 1995). This age discrepancy can probably be ascribed to several factors, including recognition of the last appearance datum of Sphaeroidinellopsis spp. and/or rapid relative decrease of $S$. seminulina vs $S$. dehiscens sensu stricto, and identification of the primitive form of $S$. dehiscens. Kučera (1998) documented survivors of $S$. sminulina to range in age to ca. $2 \mathrm{Ma}$, while Berggren et al. (1995) had earlier suggested that the development of $S$. dehiscens sensu stricto occurred in the upper part of PI3. Li et al. (2005) recorded the first occurrence of $S$. dehiscens in the South China Sea and estimated the age at ca. 3.6 Ma, which is close to that of the Sphaeroidinella event. In any case, our material does not comprise any specimens that are difficult to assign generically; the Sphaeroidinella event is documented between Samples 15 and 7 of the Yonahama Formation.

The age of the last occurrence of Dentoglobigerina altispira, which marks the top of $\mathrm{PI} 4$, was estimated at 3.09 Ma by Berggren et al. (1995) and 3.47 Ma by Gradstein et al. (2004, 2012). This level is between Sample 8 (outcrop YH7; Yonahama Formation) and Sample 9 (outcrop MB1; Minebari Formation). In other words, it is close to the boundary of the Yonahama and Minebari formations. Ujiié and Oki (1974) also recorded the last occurrence of $D$. altispira in the highest sample of the Yonahama Formation, whereas Nakagawa et al. (1976) noted it from the lowest sample of the Minebari Formation.

Oda (1978) regarded Globorotalia conoidea to be a useful mid-latitude marker, the last occurrence of which lies between that of Globorotalia margaritae and Sphaeroidinellopsis seminulina, in the lower part of PI3. Keller (1978a) also indicated that G. conoidea and Globorotalia conomiozea disap- peared slightly above the N19/N21 boundary in the central North Pacific or in N21 in the southwest Pacific, after the disappearance of Sphaeroidinellopsis spp. The last occurrence of G. conoidea, however, is found in PI4 in the present study. Consequently, we cannot regard this horizon as a useful biohorizon for correlation over large areas.

The age of the last occurrence of Globigerinoides obliquus in the Pacific region has not been assessed in detail. The last occurrence of Globigerinoides extremus was estimated at $1.98 \mathrm{Ma}$ in the South Atlantic (Gradstein et al., 2012), and this date was applied to the South China Sea by $\mathrm{Li}$ et al. (2009). The age of the last occurrence of Globoturborotalita decoraperta (Takayanagi) was estimated at $2.75 \mathrm{Ma}$ in the South Atlantic (Gradstein et al., 2012), but that of this species has not yet been well documented from the Pacific region. Ujiie and Oki (1974) reported the co-occurrence of G. obliquus, G. extremus, and G. decoraperta in an assemblage that contains Globorotalia truncatulinoides. The last occurrence datums of these species are not well established; thus, we do not use them for determining these dates.

Globorotalia tosaensis has not been found in the present study. Its first appearance is inferred to be in PI5 in the Pacific region (3.35 Ma; Saito, 1999), which covers the stratigraphical range of the present work. Why this species is absent from our material is unknown. Ujiié and Oki (1974) recorded G. tosaensis from the Minebari Formation; however, their $G$. tosaensis co-occurs with descendant G. truncatulinoides. Therefore, the first occurrence of $G$. tosaensis in Miyakojima indicates a younger date of PI4 than known to date.

Globorotalia truncatulinoides is also absent from the study material. Previously, its first appearance has been regarded to be a good indicator of the former Pliocene/Pleistocene (P/P) boundary (1.81 Ma; e.g., Blow, 1969; Bolli and Saunders, 1985). Subsequently, it was extended downwards, close to the Gauss/Matsuyama Chron (C2r/C2An, approximately $2.58 \mathrm{Ma}$ ) in the southeast Pacific (Berggren et al., 1995; Gradstein et al., 2012). It is about 0.81 myr[JENNIFER: is "myr" correct?] older than the age of P/P boundary of former definition. The level of the P/P boundary is revised as the Neogene/Quaternary boundary on the basis of the Gelasian Stage (2.58 Ma; Gibbard et al., 2010) which was once included in Pliocene. A general dating of the first occurrence datum of $G$. truncatulinoides in the northwest Pacific region has not yet been obtained, but it seems natural to assume that it was simultaneous in the North and South Pacific 
and close to the age of the newly defined P/P boundary. To explain the absence of $G$. truncatulinoides in our material, we offer two interpretations here. Firstly, our material does not cover that specific level as it does not extend to the horizon of the first occurrence of $G$. truncatulinoides by Ujiié and Ōki (1974). Their sampling location, which yielded G. truncatulinoides, is close to the Hennazaki (cape) in Miyakojima, where the uppermost part of the Minebari Formation crops out. Secondly, the appropriate oceanographic environment for $G$. truncatulinoides could not have developed, which prevented its spread during the early stage of its development. The latter explanation is supported by the absence of its ancestor, G. tosaensis. However, Tsuburaya and Sato (1985) recorded G. tosaensis and $G$. truncatulinoides from a borehole $24 \mathrm{~km}$ offshore Miyakojima. Therefore, the distribution of these two species is inferred to be geographically and stratigraphically heterogeneous. In addition, it is not known whether the age of the uppermost level in the present study is Pleistocene or not.

One sample from the Yonahama Formation in the Sugama area (southwest Miyakojima) documents zone $\mathrm{Pl} 2$, which is correlative with the lower part of the same formation in the east coast section, on the basis of the occurrence of Globorotalia margatiae and the absence of Globoturborotalita nepenthes.

\section{SYSTEMATIC PALEONTOLOGY}

The suprageneric classification of foraminifera has been debated during the last decade from the viewpoint of molecular phylogeny (e.g., Darling et al., 1997; Schweizer et al., 2008, 2009; Pawlowski et al., 2013). Such an approach is expected to lead to drastic revision of our understanding of classification. Below, we shall refrain from discussion of the taxonomy of suprageneric, and basically adopt the concept of Loeblich and Tappan (1987). Exceptions are noted in the 'Remarks' section.

Reports of Recent and fossil foraminifera from around the tropical-subtropical Pacific Ocean are too numerous to be listed here in full. Our systematic review focuses on those from around the Ryukyu Islands and includes several major monographs of material from the southwest Pacific Ocean, southeast Asia, and their marginal seas.

All specimens in the present study are stored in the collections of the National Museum of Nature and Science, Tokyo. Specimens illustrated are access numbered on mounted microslides and have the prefix of MPC.
Order FORAMINIFERIDA Eichwald, 1830 Suborder TEXTULARIINA Delage and Hérouard, 1896

Superfamily HORMOSINOIDEA Haeckel, 1894

Family HORMOSINIDAE Haeckel, 1894

Subfamily REOPHACINAE Cushman, 1910

Genus HORMOSINELLA Shchedrina, 1969 Hormosinella distans (Brady)

Figure 6.3

1881 Reophax distans Brady, p. 50.

1994 Hormosinella distans (Brady) - Loeblich and Tappan, p. 16, pl. 5, figs. 15-17.

Occurrence. A single specimen from one sample of the Yonahama Formation.

Superfamily HAPLOPHRAGMIOIDEA Eimer and Fickert, 1899

Family AMMOSPHRAEROIDINIDAE Cushman, $1927 a$

Subfamily AMMOSPHAEROIDININAE Cushman, $1927 a$

Genus AMMOSPHAEROIDINA Cushman, 1910

Ammosphaeroidina sphaeroidiniformis (Brady)

Figures 6.5, 6.6

1884 Haplophragmium sphaeroidiniforme Brady, p. 313.

1994 Ammosphaeroidina sphaeroidiniformis (Brady) - Loeblich and Tappan, p. 18, pl. 9, figs. 7-14.

Remarks. The present specimens exhibit indistinct sutures and a coarse surface, but are regarded to fall within the range of variation of the species.

Occurrence. Rare in the Yonahama Formation.

Superfamily SPIROPLECTAMMINOIDEA

Cushman, 1927a

Family SPIROPLECTAMMINIDAE Cushman, $1927 a$

Subfamily SPIROPLECTAMMININAE Cushman, $1927 a$

Genus SPIROPLECTINELA Kisel'man, 1972 Spiroplectinella wrightii (Silvestri)

Figures 6.7, 6.8

1903 Spiroplecta wrightii Silvestri, p. 59, textfigs. $1-6$.

1953 Spiroplectammina higuchii Takayanagi, p. 27, pl. 4, fig. 1.

1968 Spiroplectammina sagittula (Soldani) Huang, pl. 12, fig. 3 (non Polymorpha sagittulae Soldani, 1791 = Textularia sagittula Soldani var. soldanii Fornasini, 1883, pp. 183, 184, pl. 2, fig. 2). 


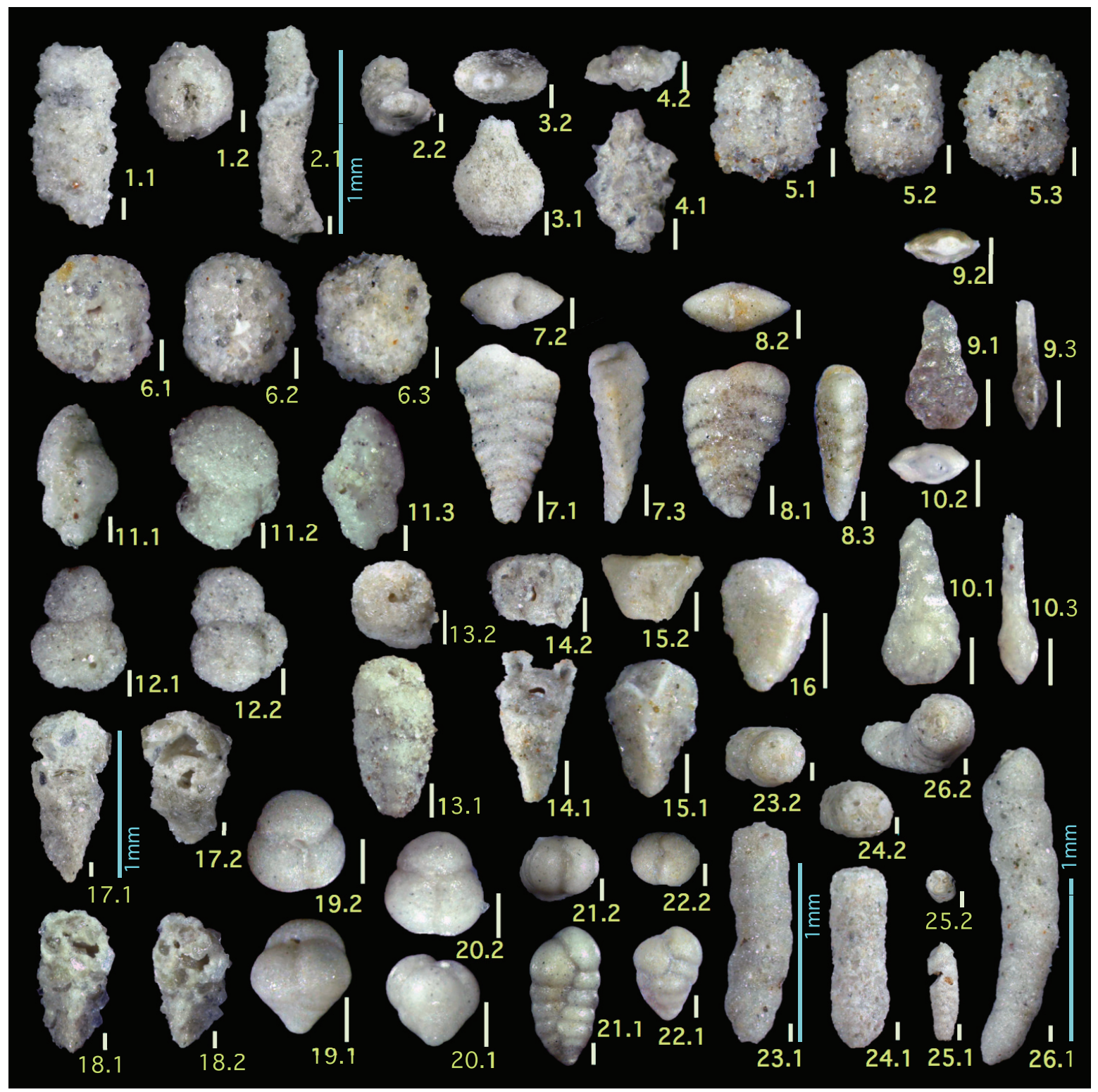

FIGURE 6. Family Hormosinidae, Ammosphaeroidinidae, Spiroplectamminidae, Duquepsamminidae, Verneuilinidae, and Eggerellidae. All scale bars $=0.1 \mathrm{~mm}$, unless otherwise indicated in 2.1, 23.1, 26.1 as $1 \mathrm{~mm}$-line. 1, 2. Rhabdammina sp., 1: MPC-26061, 2: MPC-26062, both from sample 7 (Yonahama Formation, PL4), broken specimens. 3. Hormosinella distans (Brady), MPC-26051, from sample 15 (Yonahama F., PL3), depressed specimen. 4. Reophax sp., MPC-26060, from sample 7 (Yonahama F., PL4). 5, 6. Ammosphaeroidina sphaeroidiniformis (Brady); 5: MPC-26033 from sample 6 (Yonahama F., PL2); 6: MPC-26034, from sample 6-2 (Yonahama F., PL2). 7, 8. Spiroplectinella wrightii (Silvestri), 7: MPC-26065 from sample 6-2 (Yonahama F., PL2); 8: MPC-26066 from sample MK02A-2 (Minebari F., PL5). 9, 10. Duquepsammia bulbosa (Cushman), 9: MPC-26041 from sample 8 (Yonahama F., PL4), 10: MPC-26042 from sample 15 (Yonahama F., PL3). 11, 12. Trochammina sp., 11: MPC-26077, laterally depressed specimen, 12: MPC26078, vertically depressed specimen, both from sample 7 (Yonahama F., PL4). 13, 14. Gaudryina arenaria Galloway and Wissler, 13: MPC-26045 from sample 6-2 (Yonahama F., PL2); 14: MPC26046 from sample 17 (Yonahama F., PL2). 15, 16. Gaudryina sp.A., 15: MPC-26047 from sample 3-2-low-silt (Onogoshi F., PL1); 16: MPC-26048 from sample 11 (Onogoshi F., ?PL1). 17, 18. Gaydryinella cf. japonica Asano, 17 MPC-26049, 18 MPC26050, both from sample 18 (Oura F., ?PL1). 19, 20. Eggerella bradyi (Cushman), 19: MPC-26043 from sample 14-2 (Yonahama F., PL4); 20: MPC-26044 from sample 15 (Yonahama F., PL3). 21, 22. Karreriella bradyi (Cushman), 21: MPC-26052 from sample 2 (Minebari F., PL5); 22: MPC-26053 from sample MK02A (Minebari F., PL5). 23, 24. Martinottiella communis (d'Orbigny), 23: MPC-26054 from sample 3 (Onogoshi F., PL1); 24: MPC-26055 from sample 32-low-silt (Onogoshi F., PL1). 25, 26. Martinottiella victorensis (Cushman), 25: MPC-26056 from sample 6-2 (Yonahama F., PL2); 26: MPC-26057 from sample 16 (Yonahama F., PL3). 
1987 Spiroplectinella wrightii (Silvestri) - Loeblich and Tappan, pl. 120, pl. 1-10.

1990 Spiroplectammina higuchii Takayanagi Akimoto, p. 213, pl. 14, fig. 4.

1994 Spiroplectinella wrightii (Silvestri) - Loeblich and Tappan, p. 20 , pl. 15, figs. $15-$ 18.

2002 Spiroplectinella higuchii (Takayanagi) Akimoto, Matsui, Shimokawa, and Furukawa, p. 3, pl. 1, fig. 1.

2002 Spirorutilis sp. - Kaminski, Aksu, Box, Hiscott, Filipescu, and Al-Salameen, p. 195, pl. 1, figs. 3, 4.

2012 Spiroplectinella sagittula s.I. (Defrance) Milker and Schmiedl, p. 34, figs. 9.199.21 (non Textularia sagittula Defrance, 1824, p. 177).

Diagnosis. Triangular, of moderate size for the genus, chambers not inflated, increasing slowly as added, sutures depressed, periphery bluntly angled, wall finely agglutinated, aperture low arched at base of final chamber.

Remarks. Textularia saggitula Defrance (or d'Orbigny) has been referred to as Spiroplectammina sagittula by many workers, although it is the type species of the genus Textularia and differs from S. wrightii (Loeblich and Tappan, 1987; Kaminski et al., 2002). Spiroplectammina higuchii is regarded as a synonym.

Occurrence. Common in the Yonahama and Minebari formations.

Family DUQUEPSAMMINIDAE Seiglie and Barker, 1987

Genus DUQUEPSAMMIA Seiglie and Barker, 1987 Duquepsammia bulbosa (Cushman)

Figures 6.9, 6.10

1900 Spiroplecta annectens Parker and Jones - Millett (part VII), p. 8, pl. 1, fig. 7 (non Textularia annectens Parker and Jones, 1863).

1911 Spiroplecta bulbosa Cushman, p. 5, textfig. 1.

1921 Spiroplecta bulbosa Cushman, p. 102, pl. 20, fig. 1.

1941 Spiroplectoides bulbosus (Cushman) LeRoy (Part 1), p. 31, pl. 3, figs. 95, 96.

1953 Bolivinopsis hiratai Uchio, p. 153, pl. 14, fig. 5.

1964 Bolivinopsis hiratai Uchio - LeRoy, p. F29, pl. 1, fig. 10.
1977 Spirobolivina(?) antarctica McCulloch, p. 243, 244, pl. 102, fig. 17.

1992 Spiroplectammina bulbosa (Cushman) Hatta and Ujiié (a), p. 56, pl. 1, fig. 6.

1994 Duquepsammia bulbosa (Cushman) Loeblich and Tappan, p. 20, pl. 17, figs. 5, 6.

1998 Duquepsammia bulbosa (Cushman) Hess, p. 60, pl. 8, fig. 4.

Diagnosis. Small for the genus, early planispiral stage involute, later biserial stage tends to become elongate with growth, wall finely agglutinated with much cement which results in slightly transparent wall, periphery acute.

Remarks. Textularia annectens Parker and Jones [= Spiroplectella earlandi Barker of Jones 1994, p. 50, pl. 45, figs. 22, 23 = Duquepsammina earlandi (Barker) by Loeblich and Tappan, 1994, p. 20, 21, pl. 17, figs. 1-4], which Millett (1900) referred to, is distinguished from $D$. bulbosa by its biumbilicate and evolute test in the planispiral stage. Bolivinopsis hiratai Uchio is regarded herein as a junior synonym of this species.

Occurrence. Common in the Yonahama Formation.

Superfamily VERNEUILINOIDEA Cushman, 1911

Family VERNEUILINIDAE Cushman, 1911

Subfamily VERNEUILININAE Cushman, 1911

Genus GAUDRYINA d'Orbigny, 1839a

Gaudryina arenaria Galloway and Wissler

Figures 6.13, 6.14

1927 Gaudryina arenaria Galloway and Wissler (a), p. 68 , pl. 11, fig. 5.

1950Gaudryina arenaria Galloway and Wissler Asano (Part 5), p. 1, text-figs. 1, 2.

Occurrence. Rare in the Onogoshi and Yonahama formations, common in the Minebari Formation.

Gaudryina sp. A

Figures $6.15,6.16$

Diagnosis. Small for the genus, probably juvenile specimens, characterized by finely agglutinated and smooth surface of test, chambers not inflated, sutures flush, angled periphery.

Remarks. We could not assign this form to any species. It is tentatively distinguished as "sp. A."

Occurrence. Rare to common in the Onogoshi and Yonahama formations.

Genus GAUDRYINELLA Plummer, 1931

Gaudryinella cf. japonica Asano

Figures 6.17, 6.18

Compared with: 
1950Gaudryinella japonica Asano, p. 9, pl. 2, figs. 11, 12.

Diagnosis. Large for the genus, wall coarsely agglutinated, chambers increase rapidly in size as added, test around aperture broken.

Remarks. Specimens are etched and reliable identification is difficult.

Occurrence. Two specimens from the Oura Formation.

Superfamily TEXTULARIOIDEA Ehrenberg, 1838

Family EGGERELLIDAE Cushman, 1937b

Subfamily EGGERELLINAE Cushman, 1937b

Genus EGGERELLA Cushman, 1933a Eggerella bradyi (Cushman)

Figures 6.19, 6.20

1911 Verneuilina bradyi Cushman, pp. 54, 55, text-fig. 87.

1921 Verneuilina bradyi Cushman - Cushman, p. 141, pl. 27, fig. 4.

1941 Eggerella bradyi (Cushman) - LeRoy (Part 1), p. 20, 21, pl. 2, figs. 9, 10; LeRoy (Part 2), p. 70, pl. 5, figs. 21, 22.

1964 Eggerella bradyi (Cushman) - LeRoy, p. F18, pl. 1, figs. 13, 14.

1977 Eggerella (?) cf. bradyi Cushman McCulloch, p. 587, 588, pl. 248, fig. 15.

1989 Eggerella bradyi (Cushman) - Hermelin, p. 32 , 33, pl. 2, figs. 1, 2; Inoue, p. 148, pl. 26, fig. 8.

1990 Eggerella bradyi (Cushman) - Ujiié, p. 13, pl. 2, figs. 3-5.

1994 Eggerella bradyi (Cushman) - Jones, p. 51, 52, pl. 47, figs. 4-7; Loeblich and Tappan, p. 25, pl. 28, figs. 9-14.

1995 Eggerella bradyi (Cushman) - Ujiié, p. 58, pl. 3, fig. 4.

1998 Eggerella bradyi (Cushman) - Hess, p. 60 , pl. 8, fig. 8.

Occurrence. Sporadic in the Yonahama and Minebari formations.

Genus KARRERIELLA Cushman, 1933a

Karreriella bradyi (Cushman)

Figures 6.21, 6.22

1911 Gaudryina bradyi Cushman, pp. 67, 68; fig. 107.

1932 Gaudryina bradyi Cushman - Cushman, p. 13, 14; pl. 3, figs. 8, 9.

1964 Karreriella bradyi (Cushman) - LeRoy, p. F18, 19, pl. 1, figs. 22, 23.
1968 Karreriella bradyi (Cushman) - Huang, p. 57, pl. 12, figs. 1, 2, 9, 10.

1977 Karreriella cf. bradyi Cushman - McCulloch, p. 588 pl. 248, fig. 16.

1988 Karreriella bradyi (Cushman) - Wang, Zhang, Zhao, Min, Bian, Zheng, Cheng, and Chen, p. 124, pl. 12, figs. 7-9; Marle, p. 145, pl. 5, figs. 23, 24.

1992 Karreriella bradyi (Cushman) - Kaiho, pl. 1, fig. 12.

1994 Karreriella bradyi (Cushman) - Jones, p. 50, pl. 46, figs. 1-4; Loeblich and Tappan, p. 25, 26, pl. 30, figs. 8-16.

2002 Karreriella bradyi (Cushman) - Hayward, p. 296 , pl. 1, fig. 1 .

Occurrence. Sporadic in the Minebari and Yonahama formations.

Genus MARTINOTTIELLA Cushman, 1933a Martinottiella communis (d'Orbigny)

Figures 6.23, 6.24

1826 Clavulina communis d'Orbigny, p. 268.

1846 Clavulina communis d'Orbigny, p. 196, pl. 12, figs. $1,2$.

1911 Clavulina communis d'Orbigny - Cushman, p. 72, figs. 115-117.

1921 Clavulina communis d'Orbigny - Cushman, p. 154, pl. 31, fig. 1.

1932 Clavulina communis d'Orbigny - Cushman, p. 16, pl. 4, fig. 3.

1941 Listerella communis (d'Orbigny) — LeRoy (Part 2), p. 70, pl. 1, figs. 29, 30.

1964 Schenckiella communis (d'Orbigny) LeRoy, p. F19, pl. 1, fig. 17.

1985 Martinottiella communis (d'Orbigny) Papp and Schmid, p. 74, 75, pl. 66, figs. 1-8.

1989 Martinottiella communis (d'Orbigny) Hermelin, p. 34, pl. 2, figs. 5, 6; Inoue, p. 148, 149, pl. 29, fig. 7 .

1994 Martinottiella communis (d'Orbigny) Jones, p. 52, pl. 48, figs. $1-8$.

1998 Martinottiella communis (d'Orbigny) Hess, p. 64, pl. 8, figs. 13, 14.

Occurrence. Sporadic in the Onogoshi, Yonahama, and Minebari formations.

Martinottiella victorensis (Cushman)

Figures $6.25,6.26$

1937 Listerella victorensis Cushman (a), p. 146, pl. 16, fig. 25. 
1950 Schenckiella victorensis (Cushman) Asano (Part 4), p. 4, text-figs. 24-26.

1964 Schenckiella victorensis (Cushman) LeRoy, p. F19, pl. 1, fig. 19.

Diagnosis. Mostly of moderate size, but occasionally large for the genus, characterized by longer trochospiral and biserial stages and shorter uniserial stage than Martinottiella communis.

Occurrence. Sporadic in the Minebari and Yonahama formations.

Family TEXTULARIIDAE Ehrenberg, 1838

Subfamily TEXTULARIINAE Ehrenberg, 1838 Genus BIGENERINA d'Orbigny, 1826 Bigenerina nodosaria d'Orbigny

Figures 7.1, 7.2

1826 Bigenerina nodosaria d'Orbigny, p. 261, pl. 11, figs. 9-11.

1899 Bigenerina nodosaria d'Orbigny - Millett (part VI), p. 564, pl. 7, fig. 13.

1911 Bigenerina nodosaria d'Orbigny - Cushman, p. 27, 28, figs. 46-48.

1994 Bigenerina nodosaria d'Orbigny - Jones, p. 49, pl. 44, figs. 14-18; Loeblich and Tappan, p. 27 , pl. 31 , figs. $8-12$, pl. 32 , figs. 11,12 .

Occurrence. Seven specimens from a single sample of the Onogoshi Formation; rare in the Minebari Formation.

Genus TEXTULARIA Defrance, 1824 Textularia agglutinans d'Orbigny

Figures 7.3, 7.4

1839 Textularia agglutinans d'Orbigny (a), p. 144, pl. 1, figs. 17, 18, 32, 34.

1950 Textularia agglutinans d'Orbigny - Asano (Part 3), p. 2, text-figs. 3, 4.

1959 Textularia agglutinans Orbigny - Graham and Militante, p. 26, pl. 1, figs. 18-21.

1992 Textularia agglutinans d'Orbigny - Hatta and Ujiié (a), p. 58, pl. 2, fig. 3.

1994 Textularia agglutinans d'Orbigny - Jones, p. 48, pl. 43, figs. 1-3; Loeblich and Tappan, p. 27, pl. 33, figs. 8-12.

Remarks. Our specimens have an agglutinated wall with coarse sediment particles in comparison to specimens listed above.

Occurrence. Abundant in a single sample of the Oura Formation.

Textularia cf. candeiana d'Orbigny

Figure 7.5

Compared with:
1839 Textularia candeiana d'Orbigny (a), p. 143, pl. 1, figs. 25-27.

1992 Textularia candeiana d'Orbigny - Hatta and Ujiié (a), p. 58, pl. 2, fig. 4.

Remarks. The single specimen available is etched and detailed identification is difficult.

Occurrence. Oura Formation.

Textularia pseudosolita Zheng

Figures 7.6, 7.7

1988 Textularia pseudosolita Zheng, p. 114, 321, pl. 27, fig. 5, pl. 53, fig. 5, text-fig. 32.

1992 Textularia pseudosolita Zheng - Hatta and Ujiié (a), p. 58, pl. 2, fig. 5.

1994 Textularia pseudosolita Zheng - Loeblich and Tappan, p. 29, pl. 36, figs. 5, 6, pl. 37, figs. 9-12.

Diagnosis. Moderate-sized species with broad test with curving sutures and acute periphery.

Occurrence. Rare in the Minebari and Yonahama formations.

\section{Textularia vola Lalicker and McCulloch}

Figures $7.8,7.9$

1940 Textularia vola Lalicker and McCulloch, $\mathrm{p}$. 142, 143, pl. 16, fig. 27.

Diagnosis. Moderate-sized species with depressed periphery and near-horizontal, straight sutures.

Occurrence. Rare in the Yonahama Formation.

Textularia sp. A

Figures 7.10, 7.11

Description. Relatively large, up to $1.00 \mathrm{~mm}$ in length, rhomboid in cross section; chambers slightly inflated and increasing rapidly in size as added; sutures depressed, dipping to margins; periphery subrounded; wall coarse to moderately agglutinated; aperture small, low arched, interiomarginal at base of last chamber.

Remarks. We distinguish this form tentatively as $\mathrm{sp}$. A. It is a shallow-marine inhabitant, occurring abundantly in our samples, which is why it could have been described from elsewhere, although we could not find any previous record.

Occurrence. Abundant in the Oura Formation.

Subfamily SIPHOTEXTULARIINAE Loeblich and Tappan, 1985

Genus SIPHOTEXTULARIA Finlay, 1939 Siphotextularia foliosa Zheng

Figures 7.12, 7.13

1988 Siphotextularia foliosa Zheng, pp. 126, 324, pl. 38, figs. $1,2$. 


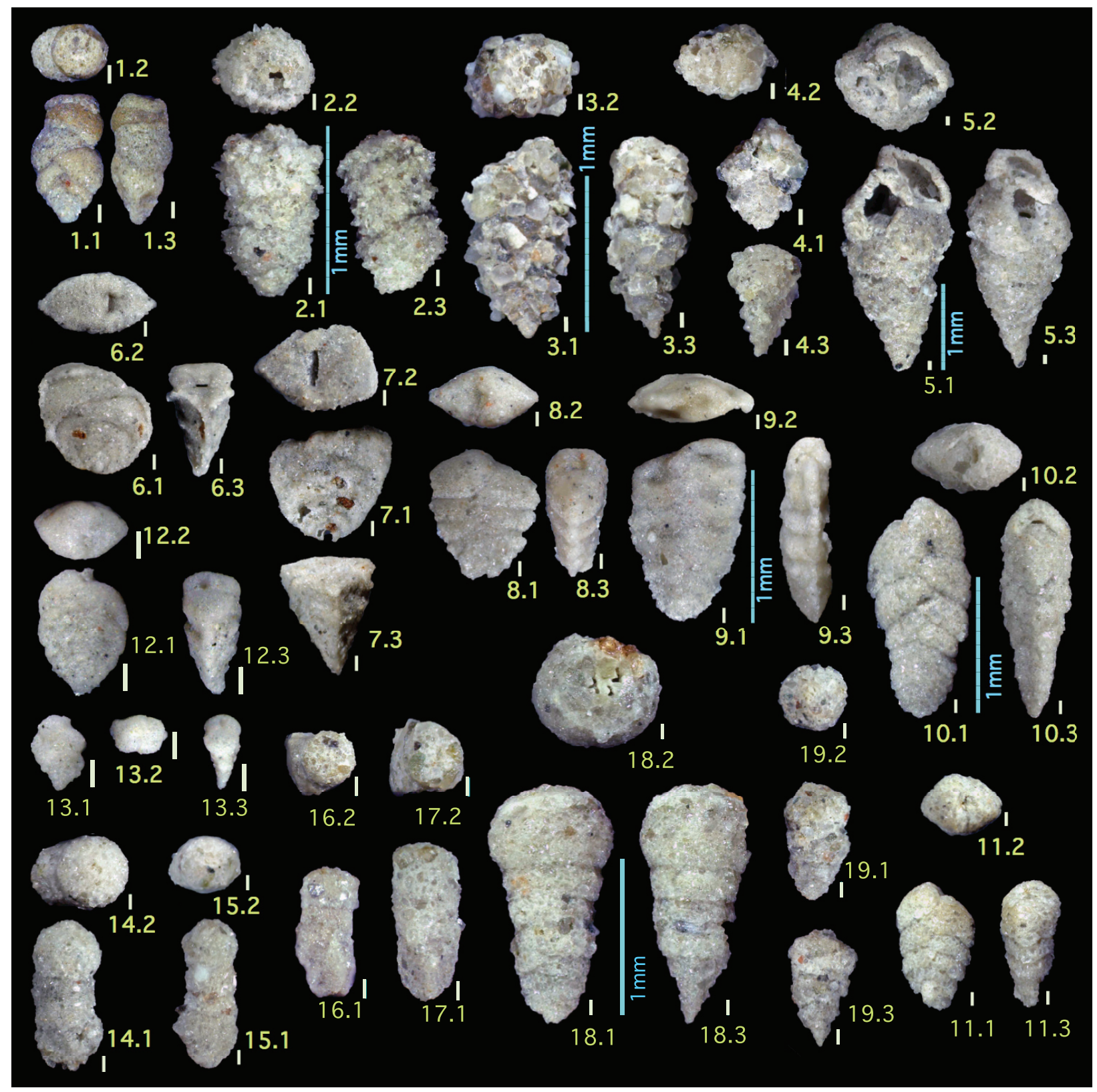

FIGURE 7. Family Textulariidae, Pseudogaudryinidae, and Valvulinidae. All scale bars $=0.1 \mathrm{~mm}$, unless otherwise indicated. 1, 2. Bigenerina nodosaria d'Orbigny, 1: MPC-26035 from sample 2 (Minebari Formation, PF5); 2: MPC26036 from sample 11 (Onogoshi F., ?PF1). Difference of coarseness and test size between specimens of figured 1 and 2 probably reflect eco-phenotypic variation and preservation. 3, 4. Textularia agglutinans d'Orbigny, 3: MPC26067, 4: MPC26068, both from sample 18 (Oura F., ?PF1). 5. Textularia cf. candeiana d'Orbigny, MPC-26069, broken specimen from sample 18 (Oura F., ?PF1). 6, 7. Textularia pseudosolita Zheng, 6: MPC-26070 from sample 6 (Yonahama F., PL2); 7: MPC-26071 from sample 6-2 (Yonahama F., PL2). 8, 9. Textularia vola Lalicker and McCulloch, 8: MPC-26072 from sample 8 (Yonahama F., PL4); 9: MPC-26073 from sample 15 (Yonahama F., PL3). $10,11$. Textularia sp.A, 10: MPC-26074, 11: MPC-26075, both from sample 18 (Oura F., ?PL1). 12, 13. Siphotextularia foliosa Zheng, 12: MPC-26063 from sample 1 (Minebari F., PL5); 13: MPC-26064 from sample 15 (Yonahama F., PL3). 14, 15. Pseudoclavulina serventyi (Chapman and Parr), 14: MPC-26058 from sample 11 (Onogoshi F., ?PL1); 15: MPC-26059 from sample 18 (Oura F., ?PL1). 16, 17. Clavulina multicamerata Chapman, 16: MPC-26037 from sample 5-2 (Minebari F., PL5); 17: MPC-26038 from sample 18 (Oura F., ?PL1). 18, 19. Cribrogoesella robusta (Brady), 18: MPC-26039, 19: MPC-26040, both from sample 18 (Oura F., ?PL1). 
1994 Siphotextularia foliosa Zheng - Loeblich and Tappan, p. 30, 31, pl. 42, figs. 1-6.

Diagnosis. Small species with inflated form, maximum width at mid-test length, with short protrusion of aperture.

Remarks. All specimens are distorted and do not retain their original morphology, but such plasticity seems to characterize this species.

Occurrence. Sporadic in the Yonahama and Minebari formations.

Family PSEUDOGAUDRYINIDAE Loeblich and Tappan, 1985

Subfamily PSEUDOGAUDRYININAE Loeblich and Tappan, 1985

Genus PSEUDOCLAVULINA Cushman, 1936

Pseudoclavulina serventyi (Chapman and Parr)

Figures 7.14, 7.15

1935 Clavulina serventyi Chapman and Parr, p. 5, pl. 1, fig. 7.

1941 Pseudoclavulina aff. anglica Cushman LeRoy (Part 1), p. 20, pl. 3, figs. 56, 57.

1992 Pseudoclavulina serventyi (Chapman and Parr) - Hatta and Ujiié (a), p. 60, pl. 3, fig. 4.

1994 Pseudoclavulina serventyi (Chapman and Parr) - Jones, p. 53, pl. 48, figs. 14-16; Loeblich and Tappan, pp. 32, 33, pl. 45, figs. $12-19$.

Remarks. LeRoy (1941, part 1) compared this species with Pseudoclavulina anglica Cushman (1936) which was originally described from the Eocene. Pseudoclavulina anglica closely resembles $P$. serventyi, and could turn out to be synonymous if the stratigraphic range of the species can be confirmed to be continuous.

Occurrence. Four specimens from the Oura Formation, a single specimen from the Onogoshi Formation.

Family VALVULINIDAE Berthelin, 1880

Subfamily VALVULININAE Berthelin, 1880

Genus CLAVULINA d'Orbigny, 1826

Clavulina multicamerata Chapman

Figures 7.16, 7.17

1907 Clavulina multicamerata Chapman, p. 127, pl. 9, fig. 5.

1994 Clavulina multicamerata Chapman Jones, p. 53, pl. 48, figs. 17, 18; Loeblich and Tappan, p. 33, 34, pl. 47, figs. 11-15.

Occurrence. Abundant in the Oura Formation, rare in the Onogoshi and Minebari formations.
Genus CRIBROGOESELLA Cushman, 1935 Cribrogoesella robusta (Brady)

Figures 7.18, 7.19

1881 Bigenerina robusta Brady, p. 53, 54.

1987 Cribrogoesella robusta (Brady) — Loeblich and Tappan, pl. 201, figs. 1-4.

1994 Cribrogoesella robusta (Brady) - Jones, pp. 49,50 , pl. 45 , figs. 9-16.

Diagnosis. Test large, growing rapidly from biserial to uniserial stages, aperture consisting of irregular fissure-like openings at top of final chamber.

Remarks. Although preservation is not good, the characteristic aperture is seen in all specimens.

Occurrence. Four specimens from the Oura Formation.

Suborder MILIOLINA Delage and Hérouard, 1896

Superfamily MILIOLOIDEA Ehrenberg, 1839

Family SPIROLOCULINIDAE Wiesner, 1920

Genus ADELOSINA d'Orbigny, 1826

Adelosina schreibersii (d'Orbigny)

Figure 8.1

1846 Quinqueloculina schreibersii d'Orbigny, $p$. 296, pl. 19, figs. 22-24.

1985 Adelosina schreibersii (d'Orbigny) - Papp and Schmid, pp. 103, 104, 106, pl. 98, figs. $6-8$, pl. 99 , figs. $1-10,13$, pl. 102 , figs. 9-14.

Occurrence. A single specimen from the Yonahama Formation.

Family HAUERINIDAE Schwager, 1876

Subfamily SIPHONAPERTINAE Saidova, 1975

Genus AMMOMASSILINA Cushman, 1933a

Ammomassilina alveoliniformis (Millett

Figures 8.2, 8.3

1898 Massilina alveoliniformis Millett (part III), p. 609 , pl. 13, figs. 5-7.

1956 Massilina alveoliniformis Millett - Asano (b), p. 65, pl. 7, fig. 11.

1959 Ammomassilina alveoliniformis (Millett) Graham and Militante, p. 33, pl. 3, fig. 1.

1994 Ammomassilina alveoliniformis (Millett) Jones, p. 24, pl. 8, fig. 13; Loeblich and Tappan, p. 45 , pl. 5 , figs. $1-5$, pl. 69 , figs. 1, 2.

1998 Ammomassilina alveoliniformis (Millett) Hess, pp. 56, 57, pl. 8, fig. 4.

Occurrence. Sporadic in the Oura, Onogoshi, and Minebari formations.

Subfamily HAUERININAE Schwager, 1876

Genus CYCLOFORINA Łuczkowska, 1972 


\section{Cycloforina rugosa (d'Orbigny)}

Figure 8.4

1826 Quinqueloculina rugosa d'Orbigny, p. 302. (?nomen nudum).

1992 Quinqueloculina rugosa d'Orbigny — Hatta and Ujiié (a), p. 68, pl. 8, fig. 6.

Remarks. Genus Cycloforina is distinguished from Quinqueloculina mainly by lack of chamber floor attaching penultimate chambers in adult specimen. Occurrence. A single specimen from the Oura Formation.

Genus MASSILINA Schlumberger, 1893 Massilina minuta Collins

Figures 8.5, 8.6

1958 Massilina minuta Collins, p. 362, pl. 3, figs. 1, 2.

1994 Massilina minuta Collins - Loeblich and Tappan, p. 47, pl. 75, figs. 7-12.

Occurrence. Two specimens from a single sample of the Yonahama Formation.

Genus QUINQUELOCULINA d'Orbigny, 1826 Quinqueloculina akneriana d'Orbigny

Figures 8.7, 8.8

1846 Quinqueloculina akneriana d'Orbigny, $\mathrm{p}$. 290 , pl. 18, figs. 16-21.

1941 Quinqueloculina akneriana d'Orbigny LeRoy (Part 2), p. 71, pl. 5, figs. 9, 10, 15, 16.

1964 Quinqueloculina akneriana d'Orbigny LeRoy, p. F19, pl. 12, figs. 13, 14.

1985 Quinqueloculina akneriana d'Orbigny Papp and Schmid, p. 100, pl. 95, figs. 15, p. 97 , pl. 91, figs. $1-4$.

Occurrence. Ten specimens from one sample of the Oura Formation.

Quinqueloculina auberiana d'Orbigny

Figures 8.9, 8.10

1839 Quinqueloculina auberiana d'Orbigny (a), p. 193, pl. 12, figs. $1-3$.

1994 Quinqueloculina auberiana d'Orbigny Jones, p. 21, pl. 5, figs. 8, 9.

Occurrence. Rare in the Yonahama Formation.

Quinqueloculina sagamiensis Asano Figure 8.11

1936 Quinqueloculina sagamiensis Asano (b), p. 612, pl. 30, fig. 5.

1956 Quinqueloculina sagamiensis Asano Asano (b), p. 61, pl. 7, fig. 16.
1964 Quinqueloculina sagamiensis Asano LeRoy, p. F19, pl. 12, figs. 17, 18.

Occurrence. A single specimen from the Yonahama Formation.

Quinqueloculina seminulum (Linnaeus)

Figures 8.12, 8.13

1758 Serpula seminulum Linnaeus, p. 786.

1944 Quinqueloculina seminulum (Linné) LeRoy (Part 2), p. 77, pl. 7, figs. 11-13.

1956 Quinqueloculina seminulum (Linné) Asano (b), pp. 61, 62, pl. 8, fig. 9, pl. 9, fig. 14.

1959 Quinqueloculina seminulum (Linné) Graham and Militante, p. 48, pl. 6, fig. 6.

1988 Quinqueloculina seminula (Linné) Wang, Zhang, Zhao, Min, Bian, Zheng, Cheng, and Chen, p. 129, pl. 14, figs. 12, 13.

1989 Quinqueloculina seminulum (Linné) Inoue, pl. 20, fig. 7, pl. 30, fig. 9.

1992 Quinqueloculina seminulum (Linnaeus) Hatta and Ujiié (a), p. 69, pl. 9, figs. 1, 2.

1994 Quinqueloculina seminulum (Linnaeus) Jones, p. 21, pl. 5, fig. 6; Akimoto, p. 285, pl. 4, fig. 12.

1998 Quinqueloculina seminula (Linné) - Hess, pp. 88, 89, pl. 9, fig. 8.

1999 Quinqueloculina seminulum (Linnaeus) Fujita, Nishi, and Saito, pl. 2, fig. 5.

2001 Quinqueloculina seminulum (Linnaeus) Kawagata, pp. 70, 71, figs. 4-10.

Occurrence. Abundant in the Oura Formation, sporadic in the Onogoshi, Yonahama, and Minebari formations.

Quinqueloculina starkeri Loeblich and Tappan

Figures 8.14, 8.15

1953 Quinqueloculina starkeri Loeblich and Tappan, p. 40, pl. 5, figs. 5-7, 9.

1989 Quinqueloculina starkeri Loeblich and Tappan - Ōki, pp. 88, 89, pl. 5, fig. 5.

1995 Quinqueloculina starkeri Loeblich and Tappan - Ujiié, p. 58, pl. 3, fig. 5.

Diagnosis. Small species with inflated chambers, thin and slightly coarse wall, depressed sutures, and wide aperture.

Occurrence. Four specimens from a single sample of the Minebari Formation.

Subfamily MILIOLINELLINAE Vella, 1957 Genus PYRGO Defrance, 1824 


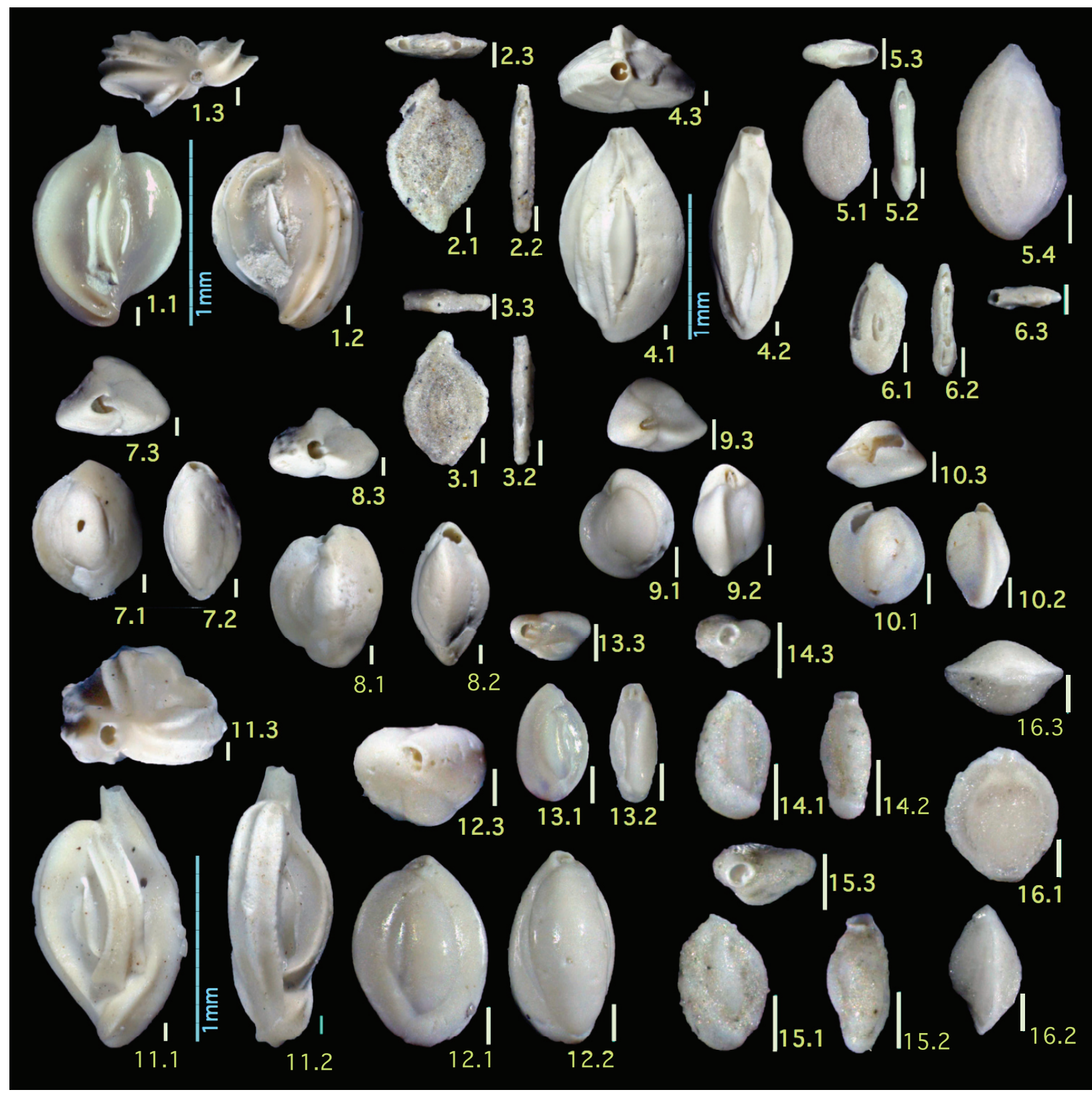

FIGURE 8. Family Spiroloculinidae and Hauerinidae. All scale bars $=0.1 \mathrm{~mm}$, unless otherwise indicated. 1. Adelosina schreibersii (d'Orbigny), MPC-26079 from sample 6 (Yonahama Formation, PL2). 2, 3. Ammomassilina alveoliniformis (Millett), 2: MPC-26080 from sample 12 (Minebari F., PL5); 3: MPC-26081 from sample MK02A (Minebari F., PL5). 4. Cycloforina rugosa (d'Orbigny), MPC-26082 from sample 18 (Oura F., ?PL1). 5, 6. Massilina minuta Collins, 5: MPC-26083, 6: MPC-26084, both from sample 14 (Yonahama F., PL4). 7, 8. Quinqueloculina akneriana d'Orbigny, 7: MPC-26088, 8: MPC-26089, both from sample 18 (Oura F., ?PL1). 9, 10. Quinqueloculina auberiana d'Orbigny, 9: MPC-26090 from sample 6 (Yonahama F., PL2); 10: MPC-26091 from sample 6-2 (Yonahama F., PL2). 11. Quinqueloculina sagamiensis Asano, MPC-26092 from sample 6-2 (Yonahama F., PL2). 12, 13. Quinqueloculina seminulum (Linnaeus), 12: MPC-26093 from sample 18 (Oura F., ?PL1); 13: MPC-26094 from sample MK02B (Minebari F., PL5). 14, 15. Quinqueloculina starkeri Loeblich and Tappan, 14: MPC-26095, 15: MPC-26096, both from sample 1 (Minebari F., PL5). 16. Pyrgo murrhina (Schwager), MPC-26085 from sample MK02A (Minebari F., PL5). 


\section{Pyrgo murrhina (Schwager)}

Figure 8.16

1866 Biloculina murrhina Schwager, p. 203, pl. 4, fig. 16.

1932 Pyrgo murrhina (Schwager) - Cushman, pp. 64,65 , pl. 15, figs. $1-3$.

1989 Pyrgo murrhina (Schwager) - Hermelin, pp. 36,37 , pl. 2, figs. $12,15,16$; Inoue, pl. 27, fig. 7.

1990 Pyrgo murrhina (Schwager) - Ujiié, p. 16, pl. 4, figs. 3-5.

1994 Pyrgo murrhina (Schwager) - Jones, pp. 18, 19, pl. 2, figs. 10, 11, 15; Loeblich and Tappan, p. 54, pl. 91, figs. $11-15$.

1998 Pyrgo murrhina (Schwager) - Hess, p. 88, pl. 9, fig. 1.

2000 Pyrgo murrhina (Schwager) - Ohkushi, Thomas, and Kawahata, p. 144, pl. 1, fig. 5.

Occurrence. A single specimen from the Minebari Formation.

\section{Pyrgo simplex (d'Orbigny)}

Figure 9.2

1846 Biloculina simplex d'Orbigny, p. 264, pl. 15, figs. 25-27.

1985 Pyrgo simplex (d'Orbigny) - Papp and Schmidt, pp. 88, 89, pl. 83, figs. 1-6.

Diagnosis. Moderate-sized species with inflated chambers resulting in near-circular cross section and rounded periphery.

Occurrence. A single specimen from the Minebari Formation.

\section{Pyrgo subsphaerica (d'Orbigny)}

Figure 9.1

1839 Biloculina subsphaerica d'Orbigny (a), p. 162, pl. 8, figs. 25-27.

1964 Pyrgo subshaerica (d'Orbigny) - LeRoy, p. F21, pl. 12, figs. 34, 35.

Occurrence. A single specimen from the Yonahama Formation.

Genus TRILOCULINA d'Orbigny, 1826

Triloculina tricarinata Parker, Jones, and Brady

Figure 9.3

1826 ?Triloculina tricarinata d'Orbigny, p. 299. (nomen nudum).

1865 Triloculina tricarinata d'Orbigny - Parker, Jones, and Brady, p. 34, pl. 1, fig. 8.

1932 Triloculina tricarinata d'Orbigny - Cushman, p. 59 , pl. 13 , fig. 3.
1941 Triloculina tricarinata d'Orbigny — LeRoy (Part 3), p. 113, pl. 1, figs. 18, 19.

1959 Triloculina tricarinata Orbigny - Graham and Militante, pp. 57, 58, pl. 8, fig. 14.

1964 Triloculina tricarinata d'Orbigny - LeRoy, p. F20, pl. 3, figs. 32, 33.

1988 Triloculina tricarinata d'Orbigny - Marle, p. 149, pl. 4, fig. 24.

1989 Triloculina tricarinata d'Orbigny - Ōki, p. 90, pl. 5, fig. 9; Hermelin, pp. 38, 39, pl. 3, figs. 6,7 .

1990 Triloculina tricarinata d'Orbigny Akimoto, p. 214, pl. 22, fig. 7; Ujiié, p. 15, pl. 3, fig. 6 .

1992 Triloculina tricarinata d'Orbigny - Hatta and Ujiié (a), pp. 75, 76, pl. 12, fig. 8.

1994 Triloculina tricarinata d'Orbigny — Loeblich and Tappan, p. 56, pl. 96, figs. 1-7.

1994 Triloculina tricarinata sensu Parker, Jones, and Brady - Jones, p. 20, pl. 3, fig. 17.

1998 Triloculina tricarinata d'Orbigny - Hess, $p$. 91, pl. 9, fig. 10.

2002 Triloculina tricarinata d'Orbigny Akimoto, Matsui, Shimokawa, and Furukawa, p. 10, pl. 20, fig. 4.

Remarks. Jones (1994) noted the taxonomic invalidity of the original description by d'Orbigny (1826) and accepted Parker et al. (1895) as authors of this taxon.

Occurrence. A single specimen from the Minebari Formation.

Subfamily SIGMOILINITINAE Łuczkowska, 1974

Genus SIGMOILINA Schlumberger, 1887 Sigmoilina cf. sigmoidea (Brady)

Figure 9.4

Compared with:

1994 Sigmoilina sigmoidea (Brady) - Jones, $p$. 18, pl. 2, figs. $1-3$.

Remarks. Due to poor preservation, detailed identification is difficult, but the sigmoidal cross section and fine white porcellaneous wall are typical features of $S$. sigmoidea.

Occurrence. A single specimen from the Yonahama Formation.

Genus SPIROSIGMOILINA Parr, 1942 Spirosigmoilina tenuis (Cžjžek)

Figures 9.5, 9.6

1848 Quinqueloculina tenuis Cžjžek, p. 149.

1964 Sigmoilinita tenuis (Cžjžek) - LeRoy, p. F20, pl. 16, figs. 32, 33. 


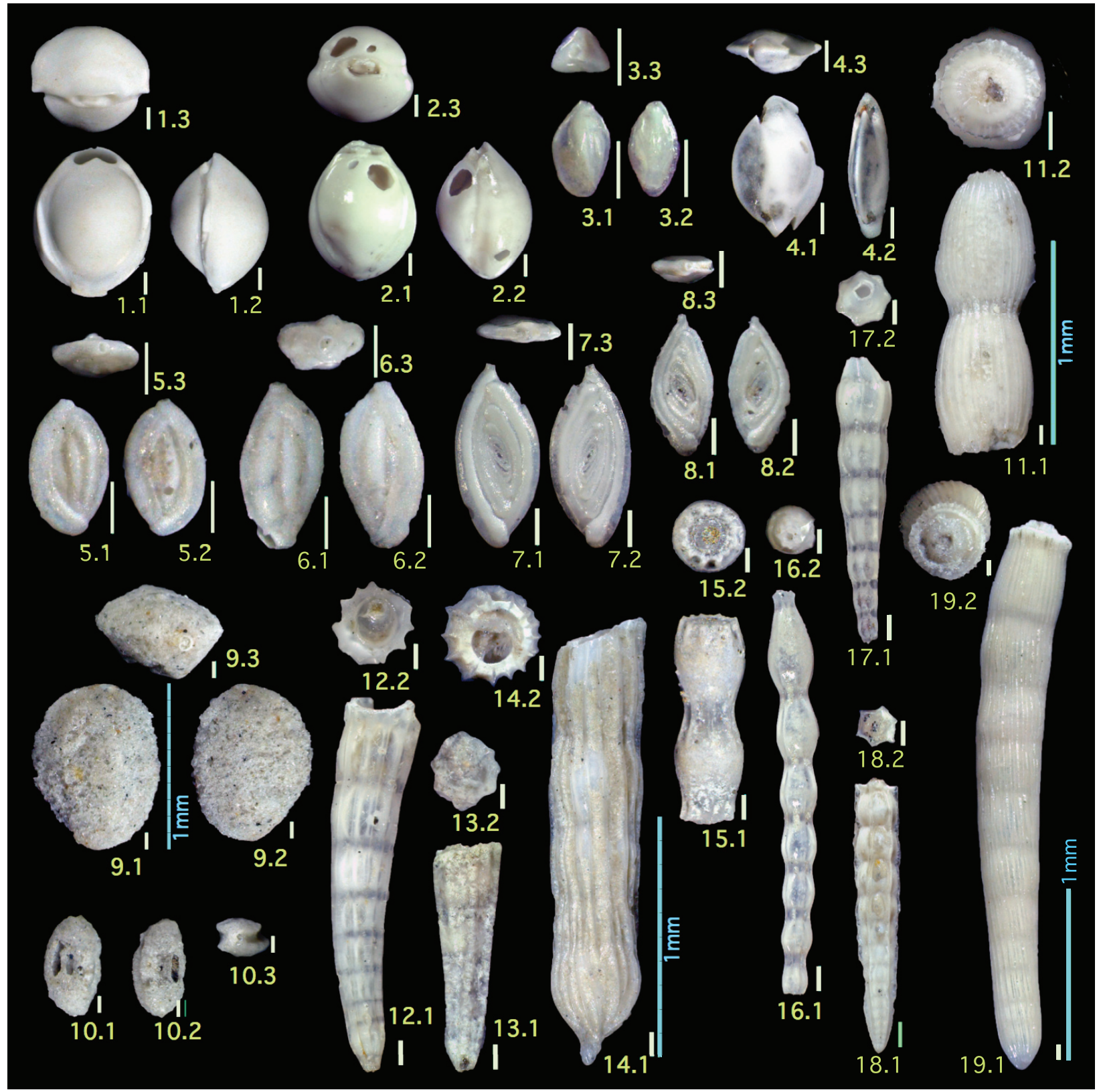

FIGURE 9. Family Hauerinidae and Nodosariidae. All scale bars $=0.1 \mathrm{~mm}$, unless otherwise indicated. 1. Pyrgo subsphaerica (d'Orbigny), MPC-26087 from sample 6-2 (Yonahama Formation, PL2). 2. Pyrgo simplex (d'Orbigny), MPC-26086 from sample MK02B (Minebari F., PL5). 3. Triloculina tricarinata Parker, Jones, and Brady (young form), MPC-26104 from sample 1 (Minebari F., PL5). 4. Sigmoilina cf. sigmoidea (Brady), MPC-26097 from sample 6 (Yonahama F., PL2), aperture broken. 5, 6. Spirosigmoilina tenuis (Cžjžek), 5: MPC-26100 from sample 11 (Onogoshi F., ?PL1); 6: MPC-26101 from sample 18 (Oura F., PL1). 7, 8. Spirosigmoilina pusilla (Earland), 7: MPC-26102 from sample 5-2 (Minebari F., PL5); 8: MPC-26103 from sample 11 (Onogoshi F., ?PL1). 9, 10. Sigmoilopsis schlumbergeri (Silvestri), 9: MPC-26098 from sample 18 (Oura F., ?PL1); 10: MPC-26099 from sample MK01 (Yonahama F., PL2). 11. Chrysalogonium equisetiformis (Schwager), MPC-26196 from sample 16 (Yonahama F., PL3). 12, 13. Dentalina albatrossi (Cushman), 12: MPC-26216, 13: MPC-26217, both from sample 2-2 (Minebari F., PL5). 14. Chrysalogonium deceptorium (Schwager), MPC-26195 from sample 2-2 (Minebari F., PL5). 15, 16. Dentalina aff. catenulata (Brady), 15: MPC-26218 from sample 6-2 (Yonahama F., PL2); 16: MPC-26219 from sample 11 (Onogoshi F., ?PL1). 17, 18. Dentalina mutsui Hada, 17: MPC-26220 from sample 18 (Oura F., ?PL1); 18: MPC-26221 from sample MK01 (Yonahama F., PL2). 19. Dentalina sp.A, MPC-26222 from sample 8-2 (Yonahama F., PL4). 
1977 Sigmoilinita tenuis (Cžjžek) - McCulloch, p. 536 , pl. 227, figs. $1,2$.

1987 Sigmoilinita tenuis (Cžjžek) - Loeblich and Tappan, pl. 35, figs. 14-18 (redrawn of Seiglie's (1965) figures).

1988 Sigmoilinita tenuis (Cžjžek) - Wang, Zhang, Zhao, Min, Bian, Zheng, Cheng, and Chen, pp. 130, 131, pl. 15, figs. 3, 4.

1994 Spirosigmoilina tenuis (Cžjžek) - Jones, p. 26 , pl. 10 , figs. $7,8,11$.

Remarks. Jones (1994) regarded the genus Spirosigmoilina as a senior synonym of Sigmoilinita Seiglie (1965); we concur.

Occurrence. Sporadic in the Oura, Onogoshi, and Yonahama formations.

Spirosigmoilina pusilla (Earland)

Figures 9.7, 9.8

1934 Spiroloculina pusilla Earland, p. 47, pl. 1, figs. 3, 4.

1921 Spiroloculina tenuissima Reuss - Cushman, p. 400, pl. 84, figs. 2, 3 (non Reuss, 1867).

1989 Opthalmidium pusillum (Earland) - Hermelin, p. 35, pl. 2, fig. 10.

1992 Opthalmidium pusillum (Earland) - Kaiho, pl. 2, fig. 3.

1994 Spirosigmoilina pusilla (Earland) - Jones, p. 26, pl. 10, figs. 9, 10.

Occurrence. Sporadic in the Onogoshi, Yonahama, and Minebari formations.

Genus SIGMOILOPSIS Finlay, 1947

Sigmoilopsis schlumbergeri (Silverstri)

Figures 9.9, 9.10

1904 Sigmoilina schlumbergeri Silvestri, p. 267.

1941 Sigmoilina schlumbergeri Silvestri LeRoy (Part 2), p. 72, pl. 7, figs. 31, 32.

1988 Sigmoilopsis schlumbergeri (Silvestri) Marle, p. 149, pl. 5, fig. 25.

1989 Sigmoilopsis schlumbergeri (Silvestri) Ōki, p. 89, pl. 5, fig. 7.

1990 Sigmoilopsis schlumbergeri (Silvestri) Akimoto, p. 212, pl. 17, fig. 8, pl. 22, fig. 8; Ujiié, p. 16, 17, pl. 3, fig. 10.

1994 Sigmoilopsis schlumbergeri (Silvestri) Jones, pp. 23, 24, pl. 8, figs. 1-4; Loeblich and Tappan, p. 59, pl. 103, figs. 912; Akimoto, p. 286, pl. 4, fig. 15.

2001 Sigmoilopsis schlumbergeri (Silvestri) Kawagata, p. 74, fig. 18.2
Occurrence. Common in all formations covered in the present study.

Suborder LAGENINA Delage and Hérouard, 1896

Superfamily NODOSARIOIDEA Ehrenberg, 1838

Family NODOSARIIDAE Ehrenberg, 1838

Subfamily NODOSARIINAE Ehrenberg, 1838

Genus CHRYSALOGONIUM Schubert, 1908

Chrysalogonium deceptorium (Schwager)

Figure 9.14

1866 Nodosaria deceptoria Schwager, p. 212, pl. 5 , fig. 30.

1941 Nodosaria spirostriolata Cushman LeRoy (Part 2), p. 75, pl. 5, fig. 23 (non Cushman, 1921).

1964 Nodosaria spirostriolata Cushman LeRoy, p. F24, pl. 15, fig. 13.

1968 Nodosaria spirostriolata Cushman Huang, p. 58, pl. 13, fig. 21.

2002 Dentalina deceptoria (Schwager) - Hayward, p. 298, pl. 2, figs. 28, 29.

2012 Chrysalogonium deceptorium (Schwager) - Hayward, Kawagata, Sabaa, Grenfell, Kerckhoven, Johnson, and Thomas, pp. 118, 119, pl. 4, figs. 9-16.

Remarks. This species has approximately 20 raised longitudinal striations, whereas Nodosaria spirostriolata Cushman has numerous (up to 4050) fine longitudinal striations.

Occurrence. A single specimen from the Minebari Formation.

\section{Chrysalogonium equisetiformis (Schwager)} Figure 9.11

1866 Nodosaria equisetiformis Schwager, p. 231, pl. 6, fig. 66.

2002 Chrysalogonium equisetiformis (Schwager) - Hayward, p. 297, pl. 1, figs. 13, 14.

2012 Chrysalogonium equisetiformis (Schwager) - Hayward, Kawagata, Sabaa, Grenfell, Kerckhoven, Johnson, and Thomas, pp. 119,120 , pl. 4 , figs. $17-22$.

Occurrence. A single specimen from the Yonahama Formation.

Genus DENTALINA Risso, 1826

Dentalina albatrossi (Cushman)

Figures 9.12, 9.13

1923 Nodosaria vertebralis (Batsch) var. albatrossi Cushman, p. 47, pl. 15, fig. 1.

1964 Nodosaria vertebralis (Batsch) var. albatrossi LeRoy, p. F25, pl. 15, fig. 12. 
1994 Dentalina albatrossi (Cushman) - Jones, p. 76 , pl. 64, figs. 11, 12, 14.

Occurrence. Two specimens from a single sample of the Minebari Formation.

Dentalina aff. catenulata (Brady)

Figures 9.15, 9.16

Compared with:

1994 Dentalina catenulata (Brady) - Jones, p. 75 , pl. 63 , figs. $32-34$.

Remarks. Specimens from Miyakojima Island have more numerous longitudinal striations than typical D. catenulata.

Occurrence. A single specimen each from the Onogoshi and Yonahama formations.

Dentalina mutsui Hada

Figures 9.17, 9.18

1931 Dentalina mutsui Hada, p. 97, text-fig. 50.

1994 Dentalina mutsui Hada - Loeblich and

Tappan, p. 63, pl. 113, figs. 5-9.

Occurrence. Sporadic in all formations covered in the present study.

Dentalina sp. A

Figure 9.19

Description. Test elongate and very slightly curved; large for the genus, up to $3.4 \mathrm{~mm}$ in length, $0.4 \mathrm{~mm}$ in diameter; chambers not inflated, almost of similar width and length, enlarging slowly as added; sutures slightly depressed; wall finely perforated; numerous longitudinal striations transverse to sutures; aperture terminal, circular, radiating slits obscured due to poor preservation.

Remarks. This form resembles Dentalina flintii (Cushman) (e.g., Jones 1994, p. 76, pl. 64, figs. 20-22) and Nodosaria spirostriolata Cushman (1917), but chambers are less inflated than in the former, and the test is slightly more curved than the latter.

Occurrence. A single partly broken specimen from the Yonahama Formation.

Genus GRIGELIS Mikhalevich, 1981

Grigelis orectus Loeblich and Tappan

Figures 10.1, 10.2

1987 Grigelis guttifera (d'Orbigny) - Loeblich and Tappan, pl. 441, figs. 2, 3 (non Dentalina guttifera d'Orbigny, 1846).

1994 Grigelis orectus Loeblich and Tappan, p. 64 , pl. 115, fig. 22

Occurrence. Two unilocular (broken) specimens from a single sample of the Onogoshi Formation.

Genus LAEVIDENTALINA Loeblich and Tappan, 1986
Laevidentalina advena (Cushman)

Figures 10.3, 10.4

1923 Nodosaria advena Cushman, p. 79, pl. 14, fig. 12.

1964 Dentalina advena (Cushman) - LeRoy, p. F23, pl. 15, fig. 31

1988 Dentalina advena (Cushman) - Marle, $p$. 141. PI. 2, fig. 11.

1994 Dentalina advena (Cushman) - Jones, $\mathrm{p}$. 74, pl. 63, fig 1.

Diagnosis. Moderate-sized species with inflated chambers and slightly curved and depressed sutures.

Remarks. Genus Laevidentalina is applied in this study for former Dentalina species lacking longitudinal costae.

Occurrence. Rare in the Minebari Formation.

Laevidentalina antarctica (Parr)

Figure 10.5

1950 Dentalina antarctica Parr, p. 329, pl. 11, figs. 26, 27

1994 Laevidentalina antarctica (Parr) - Loeblich and Tappan, p. 64, pl. 115, figs. 1113.

Occurrence. A single specimen from the Yonahama Formation.

Laevidentalina ariena (Patterson and Pettis)

Figures 10.6, 10.7

1894 Nodosaria intorta Dervieux, p. 610, pl. 5, figs. 32-34.

1938 Dentalina mucronata Neugeboren Asano (a), p. 213, pl. 25, fig. 33. (non Neugeboren, 1856).

1956 Dentalina mucronata Neugeboren Asano (a), p. 14, 15, pl. 4, figs. 27, 28. (non Neugeboren, 1856).

1986 Dentalina ariena Patterson and Pettis, new name for Nodosaria intorta Dervieux, 1894.

1994 Dentalina ariena Patterson and Pettis Jones, p. 74, pl. 62, figs. 27-31.

1990 Dentalina curta Ujiié, p. 17, pl. 4, figs. 10, 11.

1994 Laevidentalina curta (Ujiié) - Loeblich and Tappan, p. 65, pl. 115, figs. 14, 15.

Diagnosis. Small-sized species, with arched shape and flush sutures, smooth appearance.

Remarks. Japanese workers have referred to this species as $D$. mucronata, probably following the identification in the 'Challenger Report' by Brady 


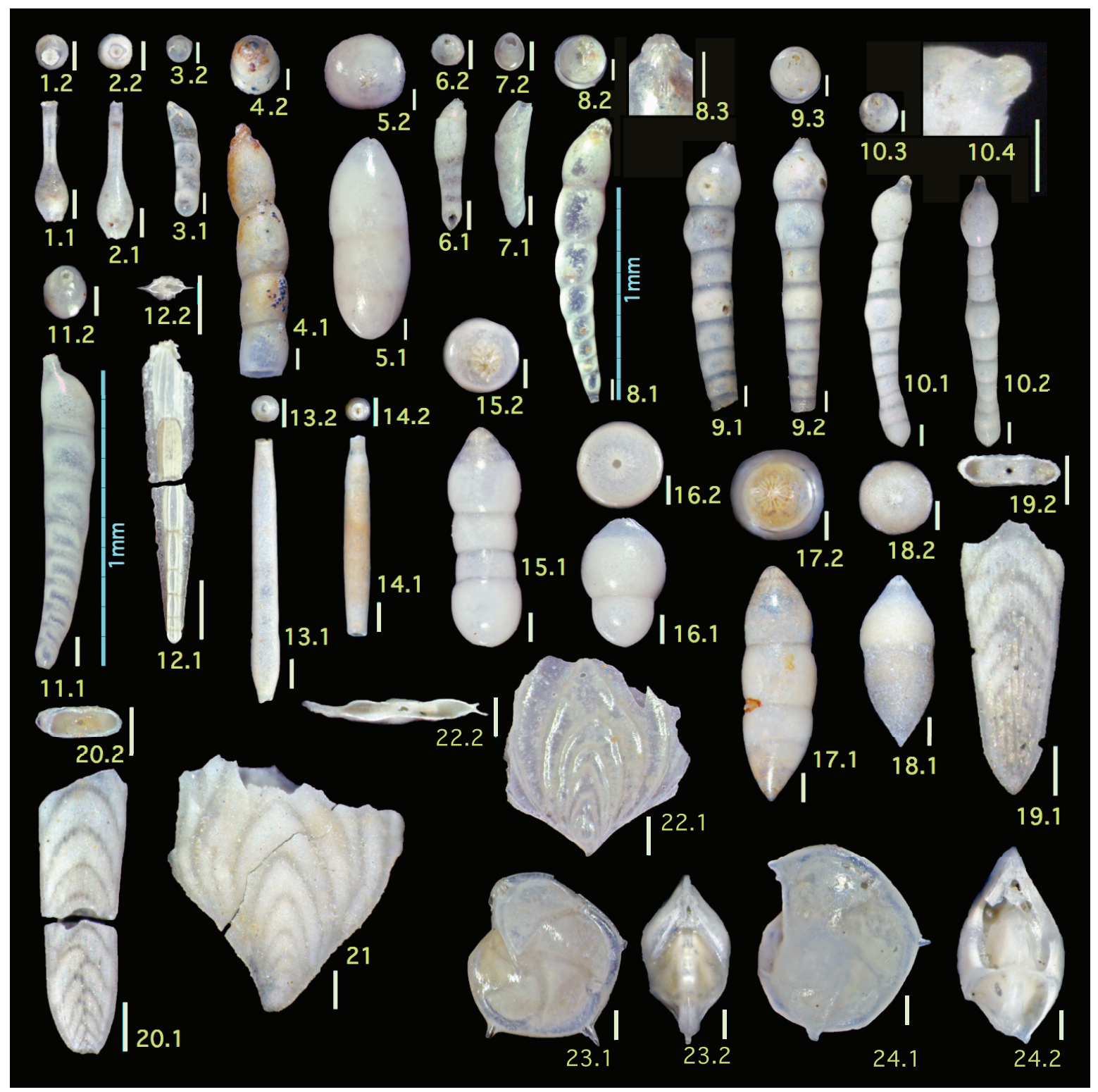

FIGURE 10. Family Nodosariidae, Stilostomellidae, and Vaginulinidae. All scale bars $=0.1 \mathrm{~mm}$, unless otherwise indicated. 1, 2. Grigelis orectus Loeblich and Tappan, 1: MPC-26294, 2: MPC-26295, both from sample 3-2-low-silt (Onogoshi Formation, PL1). 3, 4. Laevidentalina advena (Cushman), 3: MPC-26334 from sample 5 (Minebari F., PL5); 4: MPC-26335 from sample MK02A-2 (Minebari F., PL5). 5. Laevidentalina antarctica (Parr), MPC-26336 from sample 8 (Yonahama F., PL4). 6, 7. Laevidentalina ariena (Patterson and Pettis), 6: MPC-26337 from sample 3-2-low-silt (Onogoshi F., PL1); 7: MPC-26338 from sample 11 (Onogoshi F., ?PL1). 8. Laevidentalina bradyensis (Dervieux), MPC26339 from sample 1 (Minebari F., PL5); 8.3 magnified view of aperture. 9, 10. Laevidentalina subemaciata (Parr), 9: MPC-26340 from sample 3 (Onogoshi F., PL1); 10: MPC-26341 from sample 3-2-low-silt (Onogoshi F., PL1); 10.4 magnified aperture oblique-view. 11. Laevidentalina sp.A, MPC-26342 from sample 6 (Yonahama F., PL2). 12. Mucronina hasta (Parker, Jones, and Brady), MPC-26379 from sample 8-2 (Yonahama F., PL4). 13, 14. Neugeborina longiscata (d'Orbigny), 13: MPC-26547 from sample 6-2 (Yonahama F., PL2); 14: MPC-26391 from sample MK02B (Minebari F., PL5). 15-18. Pseudonodosaria discreta (Reuss), 15: MPC-26434 from sample 2-2 (Minebari F., PL5); 16: MPC-26435 from sample MK01 (Yonahama F., PL2); 17: MPC-26436 from sample 2-2 (Minebari F., PL5); 18: MPC-26437 from sample MK02A (Minebari F., PL5); 15 and 16 megalospheric forms, 17 and 18 microspheric forms. 19, 20. Parafrondicularia helenae (Chapman), 19: MPC-26418 from sample 3-2-low-silt (Onogoshi F., PL1); 20: MPC26419 from sample MK01 (Yonahama F., PL2). 21, 22. Proxifrons inaequalis (Costa), 21: MPC-26428 from sample 32-up-sand (Onogoshi F., PL1); 22: MPC-26429 from sample 6 (Yonahama F., PL2). 23, 24. Lenticulina aculeata (d'Orbigny), 23: MPC-26350 from sample 10 (Onogoshi F., PL1); 24: MPC-26351 from sample 18 (Oura F., ?PL1) 
(1884). Jones (1994) revised this to $D$. ariena; we concur. Nomenclature Nodosaria intorta Dervieux was replaced by $D$. ariena as new name by Patterson and Pettis (1986) due to homonym relationship with Dentalina intorta Terquem; the homonymy persists, since both should be assigned to the same genus Laevidentalina.

Occurrence. Sporadic in the Onogoshi, Yonahama, and Minebari formations.

Laevidentalina bradyensis (Dervieux)

Figure 10.8

1894 Nodosaria inornata d'Orbigny var. bradyensis Dervieux, p. 610, pl. 5, figs. 30 , 31.

1923 Nodosaria communis d'Orbigny - Cushman, pp. 75,76 , pl. 12 , figs. $3,4,15-17$ (non d'Orbigny, 1826)

1994 Dentalina bradyensis (Dervieux) - Jones, pp. 73, 74, pl. 62, figs. 19, 20.

1994 Laevidentalina bradyensis (Dervieux) Loeblich and Tappan, p. 64, pl. 114, figs. $1-9$, pl. 115, fig. 5 .

Occurrence. Rare in the Yonahama and Minebari formations.

Laevidentalina subemaciata (Parr)

Figures 10.9, 10.10

1950 Dentalina subemaciata Parr, p. 329, pl. 12, fig. 1.

1994 Dentalina subemaciata Parr - Jones, $\mathrm{p}$. 74, pl. 62, figs. 25, 26.

Diagnosis. Moderate-sized species with near-circular cross section and irregular chamber growth which results in preceding chambers being occasionally more inflated than later ones.

Occurrence. Sporadic in the Onogoshi and Minebari formations.

\section{Laevidentalina sp. A \\ Figure 10.11}

Description. Test elongate; very slightly curved; bilaterally compressed, and ovoid in cross section; chambers very slightly inflated; periphery rounded; sutures not depressed and gently oblique to outer arc; aperture protruding at end of test, on extension of inner arc; surface smooth and finely perforated; Initial chamber and apertural end broken.

Occurrence. A single specimen from the Yonahama Formation.
Genus MUCRONINA Ehrenberg, 1839

Mucronina hasta (Parker, Jones, and Brady)

Figure 10.12

1826 Nodosaria (les Mcronines) hasta d'Orbigny, p. 256 (nomen nudum).

1865 Nodosaria hasta d'Orbigny - Parker, Jones, and Brady, p. 27, pl. 1, fig. 29.

1987 Mucronina hasta (d'Orbigny) - Loeblich and Tappan, pl. 440, figs. $1-8$.

Remarks. This is the type species of the genus Mucronina. Only a single broken specimen is available; this shows typical features of the species including a depressed hexagonal cross section and peripheral keels.

Occurrence. A single specimen from the Yonahama Formation.

Genus PSEUDONODOSARIA Boomgaart, 1949

Pseudonodosaria discreta (Reuss)

Figures 10.15-10.18

1850 Glandulina discreta Reuss, p. 366, pl. 46, fig. 3.

1987 Pseudonodosaria discreta (Reuss) - Loeblich and Tappan, pl. 439, figs. 6-8.

1994 Pseudonodosaria discreta (Reuss) - Loeblich and Tappan, p. 66, pl. 117, figs. 1-6.

Remarks. Microspheric form showing subacute pointed tip in initial chamber; megalospheric form with inflated globular initial chamber.

Occurrence. Sporadic in the Yonahama and Minebari formations.

Subfamily PLECTOFRONDICULARIINAE Cushman, 1927a

Genus PARAFRONDICULARIA Asano, 1938a

Parafrondicularia helenae (Chapman)

Figures 10.19, 10.20

1941 Parafrodicularia helenae Chapman, pp. 154, 170, pl. 9, fig. 5.

1941 Plectofrondicularia interrupta (Karrer) LeRoy (Part 1), p. 31, pl. 3, figs. 49, 50. (non Frondicularia interrupta Karrer, 1877).

1964 Plectofrondicularia interrupta (Karrer) LeRoy, p. F29, pl. 5, fig. 25.

1994 Plectofrondicularia helenae (Chapman) Jones, p. 78, pl. 66, figs. 6, 7 .

Remarks. According to Jones (1994), P. interrupta is a junior synonym of the other valid species, $P$. helenae. Parafrondicularia japonica Asano (1938a), type species of the genus Parafrondicularia, resembles $P$. helenae, but it can be differentiated by the character of the longitudinal striations that do not extend over the sutures as clearly illus- 
trated by Aoki (1968) as Plectofrondicularia japonica (Asano). The genus Parafrondicularia is distinguished from Plectofrondicularia in having a longer biserial juvenile stage. If we regard such a feature to fall within the range of variation of the genus Plectofrondicularia as Jones (1994) did, there is the problem of homonymy between Plectofrondicularia japonica Asano (1953) and Parafrondicularia japonica Asano (1938a).

Occurrence. Rare in the Onogoshi and Yonahama formations.

\section{Genus PROXIFRONS Vella, 1963 Proxifrons inaequalis (Costa)}

Figures 10.21, 10.22

1857 Frondicularia inaequalis Costa, p. 372, pl. 3, fig. 3.

1866 Frondicularia foliacea Schwager, p. 236, pl. 6, fig. 76.

1938 Frondicularia foliacea Schwager - Asano (a), p. 118, pl. 28, fig. 16.

1964 Plectofrondicularia foliacea (Schwager) LeRoy, p. F29, pl. 11, fig. 18.

2002 Prolixifrons inaequalis (Costa) - Hayward, p. 300, pl. 2, figs. 4-9.

Remarks. All specimens are broken due to their thin wall.

Occurrence. Rare in the Yonahama and Minebari formations.

Family VAGINULINIDAE Reuss, 1860

Subfamily LENTICULININAE Chapman, Parr, and Collins, 1934

Genus LENTICULINA Lamarck, 1804

Lenticulina aculeata (d'Orbigny)

Figures 10.23, 10.24

1798 Nautilus calcar Linné varietas $\vartheta$ (theta) Fichtel and Moll, p. 79, pl. 12, figs. I, k. (non Nautilus calcar Linné, 1758, p. 709, no. 235).

1798 Nautilus calcar Linné varietas $\mu(\mathrm{mu})$ Fichtel and Moll, p. 79, pl. 13, figs. h, i.

1826 Robulina aculeana d'Orbigny, p. 289.

1923 Robulus calcar (Linné) - Cushman, pp. 7, 8, pl. 2, fig. 3.

1941 Robulus calcar (Linné) - LeRoy (Part 1), p. 24, pl. 1, figs. 88-89.

1964 Robulus calcar (Linné) - LeRoy, p. F22, pl. 4, figs. 14, 15.

1984 Lenticulina aculeata (d'Orbigny) - Rögl and Hansen, p. 56, pl. 19, fig. 3, p. 58, pl. 19, fig. 4, text-fig. 22.
1988 Lenticulina calcar (Linné) - Wang, Zhang, Zhao, Min, Bian, Zheng, Cheng, and Chen, pp. 141, 142, pl. 18, fig. 1.

1989 Lenticulina calcar (Linné) - Ōki, p. 97, pl. 7, fig. 3.

1990 Robulus calcar (Linné) - Akimoto, p. 210, pl. 15, fig. 10, pl. 17, fig. 6 .

1994 Lenticulina calcar (Linné) - Jones, pp. 81, 82, pl. 70, figs. 9-12; Loeblich and Tappan, p. 68 , pl. 120 , figs. $1-8$.

Diagnosis. Moderate-sized species with five chambers in final whorl, slightly raised sutures, and spines protruding from the middle of the peripheral keel of each chamber.

Remarks. As listed above, many workers have identified this species as $L$. calcar. According to Rögl and Hansen (1984), however, L. calcar has numerous chambers (up to seven or ten) in final whorl; while $L$. aculeata has about five, more inflated, and lobulate chambers.

Occurrence. Common in all formations studied here.

Lenticulina cultrata (de Montfort)

Figures 11.1, 11.2

1798 Nautilus calcar Linné varietas $\lambda$ (lambda) Fichtel and Moll, p. 78, pl. 13, figs. e-g.

1808 Robulus cultratus de Montfort, p. 215, figure on p. 214.

1941 Robulus aff. lucida Cushman - LeRoy (Part 1), p. 24, pl. 3, figs. 64, 65.

1951 Robulus lucidus (Cushman) - Asano (Part 15), p. 5, text-figs. 21, 22 (non Cristellaria lucida Cushman, 1923).

1956 Robulus lucidus (Cushman) - Asano (a), pp. 48,49 , pl. 1, figs. 15-18, pl. 2, figs. 5, 6. 10.

1984 Lenticulina cultrata (Montfort) - Rögl and Hansen, pp. 57, 58, pl. 16, figs. 2, 4, textfig. 21.

2001 Lenticulina cultrata (Montfort) Kawagata, pp. 74, 75, fig. 5.8.

Diagnosis. Moderate-sized species with four to five chambers in final whorl, chambers broad and inflated, sutures moderately curved and depressed, peripheral keel narrow and thin.

Remarks. This species resembles Cristellaria lucida Cushman in general shape, as identified by Asano (1951, part 15). Due to homonymy (Barker, 1960), the name is now Lenticulina atlantica (Barker). Lenticulina cultrata is distinguished from $L$. atlantica by fewer chambers per whorl that are more inflated and broader. 
Occurrence. Rare in the Onogoshi and Yonahama formations, common in the Minebari Formation.

Lenticulina depressa (Asano)

Figures 11.3, 11.4

1938 Robulus depressus Asano (a), p. 202, pl. 25 , fig. 15 , pl. 26 , figs. 10,27 , pl. 28 , fig. 11.

1956 Robulus depressus Asano - Asano (a), p. 50 , pl. 1, figs. $12-14$, pl. 2, figs. 11, 12 , pl. 3 , figs. 1,2 , pl. 6 , figs. 34,36 .

Diagnosis. Moderate- to large-sized species with significantly depressed, almost parallel-sided test, thin, well-developed peripheral keel, biumbilicate.

Remarks. Specimens of the present study exhibit fewer chambers averaging six to seven in final whorl; originally nine to eleven chambers were recorded. We interpret this as variation.

Occurrence. Sporadic in all formations of the present study.

\section{Lenticulina inornata (d'Orbigny) \\ Figure 11.5, 11.6}

1846 Robulina inornata d'Orbigny, p. 102, pl. 4, figs. 25, 26.

1985 Lenticulina inornata (d'Orbigny) - Papp and Schmid, pp. 43,44 , pl. 31, figs. $6-8$, pl. 32 , figs. $1-8$, pl. 33 , figs. $1-3$.

Occurrence. Sporadic in the Onogoshi and Minebari formations, common in the Yonahama Formation.

\section{Lenticulina iota (Cushman) \\ Figure 11.7}

1923 Cristellaria iota Cushman, p. 111, pl. 29, fig. 2, pl. 30, fig. 1.

1938 Robulus iotus (Cushman) - Asano (a), p. 202, pl. 25, figs. 7, 11, pl. 28, figs. 1, 2.

1994 Lenticulina iota (Cushman) - Jones, p. 81, pl. 70, figs. 4-6.

Occurrence. A single specimen from the Onogoshi Formation.

\section{Lenticulina orbicularis (d'Orbigny)}

Figures 11.8, 11.9

1826 Robulina orbicularis d'Orbigny, p. 288, pl. 15, figs. 8, 9.

1913 Cristellaria orbicularis (d'Orbigny) - Cushman, p. 67, pl. 36, figs. $4,5$.

1938 Robulus orbicularis d'Orbigny - Asano (a), p. 204, pl. 26, figs. 14, 18.

1941 Robulus orbicularis (d'Orbigny) - LeRoy (Part 1), p. 23, pl. 2, figs. 13, 14
1944 Robulus orbicularis (d'Orbigny) - LeRoy (Part 1), p. 17, pl. 4, figs. 15, 16.

1956 Robulus orbicularis d'Orbigny - Asano (a), pp. 49, 50, pl. 1, figs. 19-21.

1990 Robulus orbicularis d'Orbigny - Akimoto, p. 210, pl. 22, fig. 1.

1994 Lenticulina orbicularis (d'Orbigny) Jones, p. 81, pl. 69, fig. 17.

Occurrence. Sporadic in the Yonahama and Minebari formations.

Lenticulina sintikuensis Nakamura

Figures 11.10. 11.11

1937 Lenticulina sintikuensis Nakamura, p. 137, pl. 11, fig. 1.

1968 Lenticulina sintikuensis Nakamura Huang, p. 58, pl. 13, fig. 20.

Diagnosis. Moderate-sized species with thick lenticular shape, up to 10 chambers in final whorl in adult specimens, surface flush, periphery bluntly angular without keel.

Remarks. The present material exhibits less raised sutures and a more rounded periphery than the original figure of the species, but this is interpreted to be within range of variation or to have been caused by abrasion.

Occurrence. Two specimens from a single sample of the Yonahama Formation.

\section{Lenticulina thalmanni (Hessland)}

Figure 11.12

1943 Robulus thalmanni Hessland, p. 265.

1994 Lenticulina thalmanni (Hessland) - Jones, p. 81, pl. 69, fig. 13.

Remarks. The umbilical boss of the single specimen available is thicker than the typical form.

Occurrence. Minebari Formation.

Lenticulina vortex (Fichtel and Moll)

Figures 11.13, 11.14

1798 Nautilus vortex Fichtel and Moll, p. 33, pl. 2, figs. d, e.

1798 Nautilus calcar Linné varietas $\eta$ (eta) Fichtel and Moll, p. 76, pl. 12, figs. g-i.

1913 Cristellaria vortex (Fichtel and Moll) Cushman, p. 68, pl. 32, fig. 3.

1933 Robulus vortex (Fichtel and Moll) - Cushman (c), pp. 5, 6, pl. 2, fig. 1.

1964 Robulus vortex (Fichtel and Moll) LeRoy, p. F21, pl. 4, figs. 7, 8.

1984 Lenticulina vortex (Fichtel and Moll) Rögl and Hansen, p. 30, pl. 2, figs. 3, 4, text-fig. 8, pl. 19, figs. 1, 2. 


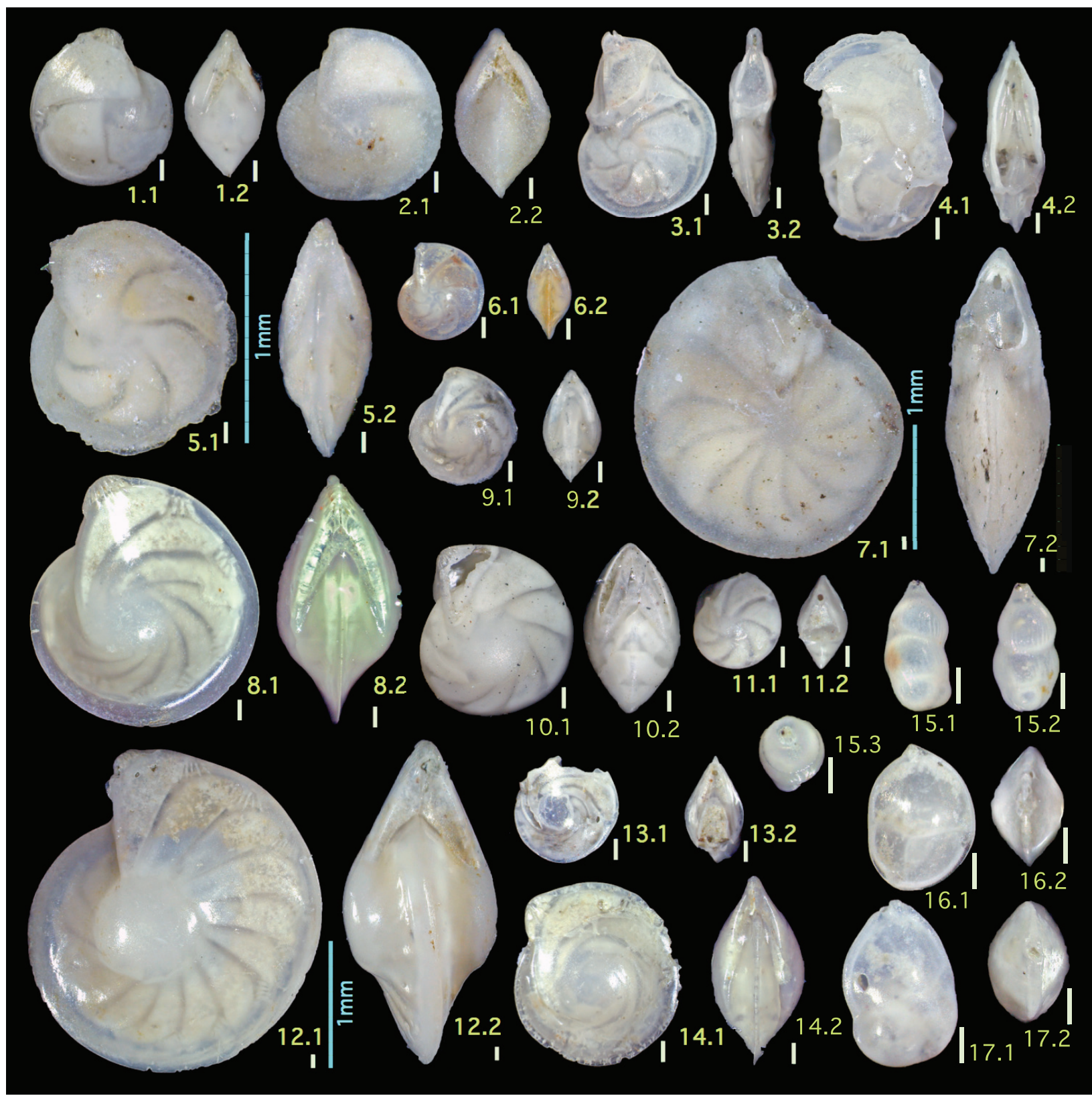

FIGURE 11. Family Vaginulinidae. All scale bars $=0.1 \mathrm{~mm}$, unless otherwise indicated. 1, 2. Lenticulina cultrata (de Montfort), 1: MPC-26352 from sample 2 (Minebari Formation, PL5); 2: MPC-26353 from sample MK02A-2 (Minebari F., PL5). 3, 4. Lenticulina depressa (Asano), 3: MPC-26354 from sample 11 (Onogoshi F., ?PL1); last three chambers show aberrant inflation; 4: MPC-26355 from sample 18 (Oura F., ?PL1) broken specimen. 5, 6. Lenticulina inornata (d'Orbigny), 5: MPC-26356 from sample 3-2-up-sand (Onogoshi F., PL1); 6: MPC-26357 from sample MK02B (Minebari F., PL5). 7. Lenticulina iota (Cushman), MPC-26358 from sample 3-2-up-sand (Onogoshi F., PL1). 8, 9. Lenticulina orbicularis (d'Orbigny), 8: MPC-26359 from sample 6 (Yonahama F., PL2); 9: MPC-26360 from sample MK01 (Yonahama F., PL2). 10, 11. Lenticulina sintikuensis Nakamura, 10: MPC-26361, 11: MPC-26362, both from sample 6-2 (Yonahama F., PL2). 12. Lenticulina thalmanni (Hessland), MPC-26363 from sample 2-2 (Minebari F., PL5). 13, 14. Lenticulina vortex (Fichtel and Moll), 13: MPC-26364 from sample 2-2 (Minebari F., PL5); 14: MPC-26365 from sample MK02A-2 (Minebari F., PL5); 13, broken specimen. 15. Marginulinopsis striatulus (Cushman), MPC-26369 from sample 4 (Minebari F., PL5). 16, 17. Neolenticulina variabilis (Reuss), 16: MPC-26389 from sample 1-2 (Minebari F., PL5); 17: MPC-26390 from sample 18 (Oura F., ?PL1). 
1985 Lenticulina vortex (Fichtel and Moll) Papp and Schmid, p. 44, pl. 33, figs. 4-8.

1994 Lenticulina vortex (Fichtel and Moll) Jones, p. 81, pl. 69, figs. 14-16; Loeblich and Tappan, pp. 68, 69, pl. 121, figs. 914.

Occurrence. Sporadic in the Yonahama and Minebari formations.

Genus MARGINULINOPSIS Silvestri, 1904 Marginulinopsis striatulus (Cushman)

Figure 11.15

1913 Marginulina striatula Cushman, p. 79, pl. 23, fig. 4.

1956 Marginulina striatula Cushman - Asano (a), p. 14, pl. 4, fig. 12

1964 Marginulina striatula Cushman - LeRoy, p. F22, pl. 5, fig. 11.

Occurrence. A single specimen from the Minebari Formation.

Genus NEOLENTICULINA McCulloch, 1977 Neolenticulina variabilis (Reuss)

Figures 11.16, 11.17

1850 Cristellaria variabilis Reuss, p. 369, pl. 46, figs. 15, 16.

1866 Cristellaria peregrina Schwager, p. 245, pl. 7, fig. 89.

1903 Cristellaria variabilis Reuss - Millett (part XIV), pp. 256, 257, pl. 5, fig. 1.

1913 Cristellaria variabilis Reuss - Cushman, p. 70 , pl. 36, figs. $1-3$.

1938 "Cristellaria" peregrina Schwager - Asano (a), pl. 29, figs. 6, 7, 11.

1956 Lenticulina peregrina (Schwager) Asano (a), p. 7, pl. 3, figs. 9, 17, 18.

1964 Lenticulina peregrina (Schwager) LeRoy, p. F22, pl. 4, figs. 5, 6.

1968 Lenticulina peregrina (Schwager) Huang, p. 58, pl. 13, fig. 35.

1977 Neolenticulina chathamensis McCulloch, p. 8, pl. 94, fig. 12.

1994 Neolenticulina variabilis (Reuss) - Jones, p. 80 , pl. 68 , figs. $11-16$.

1994 Neolenticulina peregrina (Schwager) Loeblich and Tappan, p. 69, pl. 124, figs. $1-11$.

Remarks. Jones (1994) demonstrated that N. variabilis has precedence over $L$. peregrina; we adopt this view.
Occurrence. Abundant in the Oura Formation, rare in the Onogoshi and Minebari formations.

Genus SARACENARIA Defrance, 1824

Saracenaria aff. akitaensis Iwasa and Kikuchi

Figures 12.1, 12.2

Compared with:

1954 Saracenaria akitaensis Iwasa and Kikuchi, p. 191, text-fig. 3.

Diagnosis. Species with rounded triangular shape in cross section and smooth surface without depressed sutures and inflation of chambers.

Remarks. In general shape, this form closely resembles $S$. akitaensis originally described from the middle Miocene of northern Japan. Specimens from Miyakojima, however, have a smaller test size (about $0.6 \mathrm{~mm}$ in diameter) than S. akitaensis (1.5 $\mathrm{mm}$ in the original description).

Occurrence. Rare in the Yonahama and Minebari formations.

\section{Saracenaria altifrons (Parr)}

Figure 12.3

1994 Saracenaria altifrons (Parr) - Jones, $p$. 113, pl. 114, fig. 17.

Occurrence. A single specimen from the Yonahama Formation.

\section{Saracenaria italica Defrance}

Figures $12.4,12.5$

1824 Saracenaria italica Defrance, p. 177.

1913 Cristellaria italica (Defrance) - Cushman, p. 78 , pl. 33 , fig. 3 .

1921 Cristellaria italica (Defrance) - Cushman, pp. 252, 253, pl. 51, fig. 2.

1941 Saracenaria italica (Defrance) - LeRoy (Part 1), p. 28, pl. 1, figs. 53, 54; LeRoy (Part 2), p. 76, pl. 7, figs. 21-24.

1944 Saracenaria italica (Defrance) - LeRoy (Part 1), p. 21, pl. 1, fig. 24, pl. 5, pl. 5, fig. 18; LeRoy (Part 2), p. 81, 82, pl. 2, fig. 12.

1964 Saracenaria italica Defrance - LeRoy, p. F25, pl. 3, figs. 29, 30.

1987 Saracenaria italica Defrance - Loeblich and Tappan, pl. 448, figs. 16, 17.

1988 Saracenaria italica Defrance - Wang, Zhang, Zhao, Min, Bian, Zheng, Cheng, and Chen, p. 140, pl. 18, fig. 2.

1994 Saracenaria italica Defrance - Jones, p. 80 , pl. 68, figs. 18, 20-23; Loeblich and Tappan, p. 69, pl. 125, figs. 9-16.

Occurrence. Two specimens from a single sample of the Minebari Formation. 


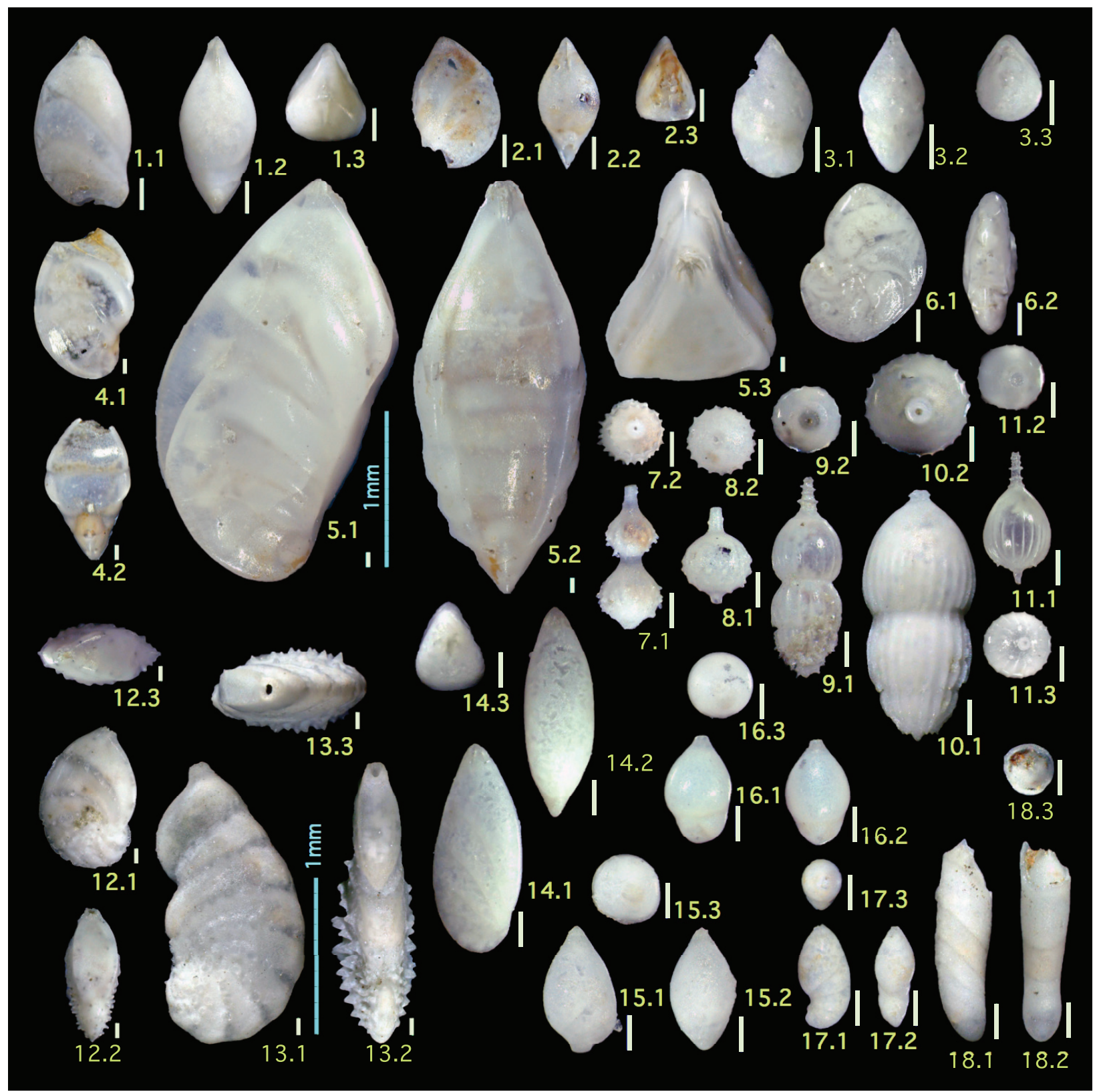

FIGURE 12. Family Vaginulinidae. All scale bars $=0.1 \mathrm{~mm}$, unless otherwise indicated. 1, 2. Saracenaria aff. akitaensis Iwasa and Kikuchi, 1: MPC-26485 from sample 2 (Minebari Formation, PL5); 2: MPC-26486 from sample 17 (Yonahama F., PL2). 3. Saracenaria altifrons (Parr), MPC-26487 from sample 15 (Yonahama F., PL3). 4, 5. Saracenaria italica Defrance, 4: MPC-26488, 5: MPC-26489, both from sample 2-2 (Minebari F., PL5). 6. Spincterules sp.A, MPC-26507 from sample 5-2 (Minebari F., PL5). 7, 8. Amphicoryna hispida (d'Orbigny), 7: MPC-26118 from sample 3-2-low-silt (Onogoshi F., PL1); 8: MPC-26119 from sample MK01 (Yonahama F., PL2). 9-11. Amphicoryna separans (Brady), 9: MPC-26120 from sample 3-2-low-silt (Onogoshi F., PL1); 10: MPC-26121 from sample 14-2 (Yonahama F., PL4), 11: MPC-26122 from sample 5 (Minebari F., PL5). 12, 13. Astacolus japonicus (Asano), 12: MPC26132 from sample 2 (Minebari F., PL5); 13: MPC-26133 from sample 6-2 (Yonahama F., PL2). 14. Astacolus insolitus (Schwager), MPC-26131 from sample 15 (Yonahama F., PL3). 15, 16. Hemirobulina aff. obesa (Cushman), 15: MPC-26546 from sample 16 (Yonahama F., PL3); 16: MPC-26315 from sample MK01 (Yonahama F., PL2). 17. Hemirobulina aff. uedai (Asano), MPC-26316 from sample 15 (Yonahama F., PL3). 18. Vaginulinopsis sublegumen Parr, MPC-26528 from sample 3 (Oura F., PL1). 


\section{Genus SPINCTERULES Montfort, 1808 \\ Spincterules sp. A \\ Figure 12.6}

Description. Test planispiral, of moderate size (about $0.5 \mathrm{~mm}$ in diameter); bilaterally compressed and with near-parallel sides; six chambers in last whorl; sutures curve backwards; umbilicus slightly raised by thickened wall; surface smooth, flushed; suture slightly raised but obscured by abrasion; aperture radiate.

Remarks. Mainly due to poor preservation, we were unable to identify this to species level.

Occurrence. A single specimen from the Minebari Formation.

Subfamily MARGINULININAE Wedekind, 1937 Genus AMPHICORYNA Schlumberger in MilneEdwards, 1881 Amphicoryna hispida (d'Orbigny)

Figures 12.7, 12.8

1846 Nodosaria hispida d'Orbigny, p. 35, pl. 1, figs. 24, 25.

1964 Nodosaria hirsuta (d'Orbigny) — LeRoy, p. F24, pl. 15, fig. 3.

1994 Amphicoryna hirsuta (d'Orbigny) - Jones, p. 75 , pl. 63 , figs. $12-15$.

1994 Nodosaria hispida d'Orbigny - Loeblich and Tappan, p. 65, pl. 116, figs. 7, 8.

Remarks. We follow Loeblich and Tappan (1994) in considering the species hirsuta to be invalid, and Jones (1994) in generic assignment.

Occurrence. Sporadic in the Onogoshi, Yonahama, and Minebari formations.

Amphicoryna separans (Brady)

Figures 12.9-12.11

1884 Nodosaria scalaris var. separans Brady, p. 511, pl. 64, figs. 16-19.

1902 Nodosaria scalaris var. separans Brady Millett (part XIII), pp. 520, 521, pl. 11, figs. $11,12$.

1913 Nodosaria scalaris (Batsch) - Cushman, p. 58 , pl. 24, fig. 7 .

1941 Lagenonodosaria scalaris (Batsch) LeRoy (Part 1), p. 28, pl. 2, figs. 30, 31; LeRoy (Part 2), p. 77, pl. 2, fig. 18.

1944 Lagenonodosaria scalaris (Batsch) LeRoy (Part 1), p. 21, pl. 8, fig. 12.

1956 Lagenonodosaria separans (Brady) Asano (a), p. 28, pl. 6, figs. 8, 9.

1964 Lagenonodosaria scalaris (Batsch) LeRoy, p. F27, pl. 15, figs. 20, 29.
1968 Amphicoryna scalaris (Batsch) - Huang, p. 55, pl. 13, fig. 12.

1988 Amphicoryna scalaris (Batsch) - Wang, Zhang, Zhao, Min, Bian, Zheng, Cheng, and Chen, p. 139, pl. 17, figs. 17, 19; Marle, p. 139, pl. 4, fig. 22.

1989 Amphicoryna scalaris (Batsch) - Ōki, pp. 92, 93, pl. 6, fig. 3.

1990 Amphicoryna scalaris (Batsch) - Akimoto, p. 191, pl. 17, fig, 7 .

1992 Amphicoryna scalaris (Batsch) - Hatta and Ujiié (b), p. 166, pl. 21, fig. 8.

1994 Amphicoryna scalaris (Batsch) - Jones, p. 75 , pl. 63 , figs. $29-31$.

1994 Amphicoryna separans (Brady) - Jones, p. 76, pl. 64, figs. 16-19; Loeblich and Tappan, p. 71, pl. 127, figs. 1-18.

1998 Amphicoryna scalaris (Batsch) - Hess, p. 76, pl. 12, fig. 2.

Remarks. This is one of the commoner bathyal species in the waters around Japan. The protruding neck of $A$. separans is annulated, whereas that of $A$. scalaris is smooth and elongate; for this reason, previous records of $A$. scalaris from Japan are in fact $A$. separans as listed, in part, in the synonymy by Loeblich and Tappan (1994). Separation of chambers is not a prime feature in the identification of this species, irrespective of the specific name. In addition, we here show a broken and separated unilocular specimen to illustrate that such specimens may induce erroneous identification as a unilocular species.

Occurrence. Common in all the formations of the present study.

Genus ASTACOLUS de Montfort, 1808 Astacolus insolitus (Schwager) Figure 12.14

1866 Cristellaria insolita Schwager, p. 242, pl. 6, fig. 85.

1992 Astacolus insolitus (Schwager) - Hatta and Ujiié (b), p. 166, pl. 21, fig. 9.

Occurrence. A single specimen from the Yonahama Formation.

\section{Astacolus japonicus (Asano)}

Figures 12.12, 12.13

1936 Lenticulina japonica Asano (c), p. 328, pl. 37, fig. 7.

1938 Planularia japonica (Asano) - Asano (a), pp. 205,206 , pl. 24 , figs. 13,14 , pl. 26 , fig. 7. 
1964 Hemicristellaria japonica (Asano) LeRoy, p. F24, pl. 5, fig. 17.

1988 Planularia japonica (Asano) - Wang, Zhang, Zhao, Min, Bian, Zheng, Cheng, and Chen, p. 140, pl. 18, figs. 5, 6.

1994 Astacolus japonicus (Asano) - Loeblich and Tappan, p. 72, pl. 130, figs. 14-19.

Occurrence. Sporadic in all formations of the present study.

Genus HEMIROBULINA Stache, 1864

Hemirobulina aff. obesa (Cushman)

Figures 12.15, 12.16

Compared with:

1923 Marginulina glabra var. obesa Cushman, p. 128, pl. 37, fig. 1.

1990 Marginulina cf. obesa (Cushman) - Ujiié, pp. 20, 21, pl. 6, figs. 2, 3 .

1994 Marginulinopsis sp. - Loeblich and Tappan, p. 69, pl. 124, figs. 16, 17.

Remarks. This species closely resembles Hemirobulina obesa in general test shape, whereas test length of all specimens of in the present material is approximately $0.3 \mathrm{~mm}$, i.e., much smaller than $H$. obesa which attains lengths of up to $3 \mathrm{~mm}$.

Occurrence. Sporadic in the Yonahama and Minebari formations.

Hemirobulina aff. uedai (Asano)

Figure 12.17

Compared with:

1938 Marginulina uedai Asano (a), p. 210, pl. 27, figs. 8, 9.

Diagnosis. Small species with oval cross section, inflated chambers in uncoiled stage.

Remarks. This species is similar to Hemirobulina uedai in test shape, but is much smaller (about 0.3 $\mathrm{mm}$ in length), whereas the type of $H$. uedai measures $1.5 \mathrm{~mm}$.

Occurrence. A single specimen from the Yonahama Formation.

Genus VAGINULINOPSIS Silvestri, 1904

Vaginulinopsis sublegumen Parr

Figure 12.18

1950 Vaginulinopsis sublegumen Parr, p. 325, pl. 11, fig. 18.

1994 Vaginulinopsis sublegumen Parr - Loeblich and Tappan, p. 74, pl. 131, figs. 12, 13, pl. 133, figs. $10-17$.

Occurrence. Two specimens from a single sample of the Onogoshi Formation.
Vaginulinopsis tenuis (Bornemann)

Figures 13.1, 13.2

1855 Marginulina tenuis Bornemann, p. 326, pl. 13, fig. 4.

1921 Cristellaria tenuis (Bornemann) - Cushman, p. 250, pl. 50, fig. 2.

1956 Marginulina tenuis Bornemann - Asano (a), p. 13, pl. 4, figs. 9, 10.

1964 Vaginulina tenuis (Bornemann) - LeRoy, p. F25, pl. 3, fig. 13.

1994 Vaginulinopsis tenuis (Bornemann) Jones, p. 78, pl. 66, figs. 17, 21-23.

Occurrence. Two specimens from a single sample of the Minebari Formation.

Subfamily VAGINULININAE Reuss, 1860

Genus PLANULARIA Defrance in de Blainville, 1826

Planularia californica (Galloway and Wissler)

Figures 13.3, 13.4

1927 Astacolus californicus Galloway and Wissler (a), p. 46, pl. 8, fig. 4.

1994 Planularia californica (Galloway and Wissler) - Loeblich and Tappan, p. 75, pl. 130, fig. 11, pl. 133, figs. $1-9$.

Occurrence. Two specimens from a single sample of the Minebari Formation.

\section{Genus VAGINULINA d'Orbigny, 1826 Vaginulina bradyi Cushman}

Figures 13.5, 13.6

1917 Vaginulina bradyi Cushman, p. 661.

1921 Vaginulina bradyi Cushman - Cushman, p. 260, pl. 42, figs. 3, 4 .

1941 Vaginulina bradyi Cushman - LeRoy (Part 2), p. 76, pl. 7, figs. 25.

1944 Vaginulina bradyi Cushman - LeRoy (Part 1), p. 21, pl. 1, fig. 26.

1954 Vaginulina bradyi Cushman - Tai, pl. 1, fig. 2.

1994 Vaginulina bradyi Cushman - Loeblich and Tappan, p. 75, pl. 134, figs. 6, 7, 1419.

Occurrence. A single specimen each from the Yonahama and Minebari formations.

Family LAGENIDAE Reuss, 1862b

Genus CONOLAGENA Malumián, Náñez, and Caramés, 1991

Conolagena favosopunctata (Brady)

Figure 13.7

1881 Lagena favoso-punctata Brady, p. 62. 


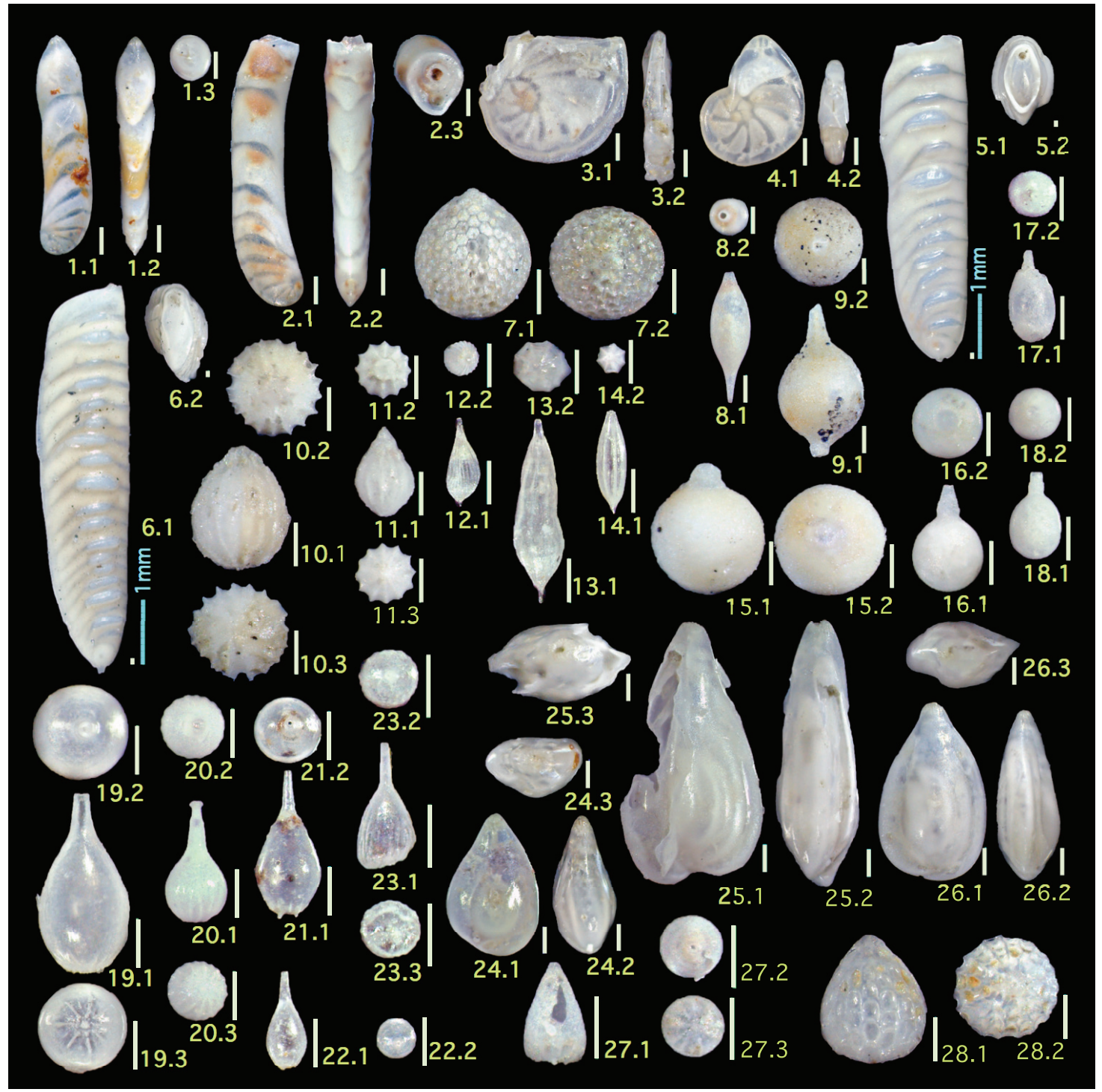

FIGURE 13. Family Vaginulinidae, Lagenidae, Polymorphinidae, and Ellipsolagenidae. All scale bars $=0.1 \mathrm{~mm}$, unless otherwise indicated. 1, 2. Vaginulinopsis tenuis (Bornemann), 1: MPC-26529, 2: MPC-26530, both from sample 2-2 (Minebari Formation, PL5). 3, 4. Planularia californica (Galloway and Wissler), 3: MPC-26414, 4: MPC26415, both from sample 2-2 (Minebari F., PL5). 5, 6. Vaginulina bradyi Cushman, 5: MPC-26526 from sample 4 (Minebari F., PL5); 6: MPC-26527 from sample MK01 (Yonahama F., PL2). 7. Conolagena favosopunctata (Brady), MPC-26209 from sample MK02A-2 (Minebari F., PL5). 8. 9. Hyalinonetrion gracillium (Costa), 8: MPC-26332 from sample 6-2 (Yonahama F., PL2); 9: MPC-26333 from sample MK02A-2 (Minebari F., PL5). 10, 11. Lagena sulcata (Walker and Jacob), 10: MPC-26343 from sample 3-2-up-sand (Onogoshi F., PL1); 11: MPC-26344 from sample 17 (Yonahama F., PL2). 12. Procerolagena gracilis (Williamson), MPC-26425 from sample 14-2 (Yonahama F., PL4). 13, 14. Procerolagena multilatera (McCulloch), 13: MPC-26426 from sample 8-2 (Yonahama F., PL4); 14: MPC26427 from sample 14 (Yonahama F., PL4). 15, 16. Pygmaeoseistron aspera (Reuss), 15: MPC-26446 from sample 3-2-up-sand (Onogoshi F., PL1); 16: MPC-26447 from sample 16 (Yonahama F., PL3). 17, 18. Pygmaeoseistron hispidulum (Cushman), 17: MPC-26448 from sample 1 (Minebari F., PL5); 18: MPC-26449 from sample 15 (Yonahama F., PL3). 19-22. Pygmaeoseistron oceanicum (Albani), 19: MPC-26450 from sample 5 (Minebari F., PL5); 20: MPC26451 from sample 7 (Yonahama F., PL4); 21: MPC-26549 from sample 11 (Onogoshi F., ?PL1), 22: MPC-26550 from sample 17 (Yonahama F., PL2). 23. Pygmaeoseistron sp. indet. MPC-26452 from sample 10 (Onogoshi F., PL2). 24-26. Sigmoidella elegantissima (Parker and Jones), 24: MPC-46490 from sample 2-2 (Minebari F., PL5); 25: MPC-46491 and 26: MPC-46492 from sample 18 (Oura F., ?PL1). 27. Exsculptina glaphyraheda Loeblich and Tappan, MPC-26254 from sample 17 (Yonahama F., PL2). 28. Favulina melo (d'Orbigny), MPC-26257 from sample 4-2 (Minebari F., PL5). 
1977 Lagena punctatiformis McCulloch, p. 43, pl. 54, fig. 18.

1994 Oolina favosopunctata (Brady) - Jones, p. 66 , pl. 58, fig. 35.

1994 Conolagena favosopunctata (Brady) Loeblich and Tappan, p. 76, 77, pl. 137, figs. $1-8$.

Remarks. The genus Conolagena was erected subsequent to the summary of generic lagenid classification by Loeblich and Tappan (1987).

Occurrence. A single specimen from the Minebari Formation.

Genus HYALINONETRION Patterson and

Richardson in Loeblich and Tappan, 1987 Hyalinonetrion gracilium (Costa)

Figures 13.8, 13.9

1856 Amphorina gracillis Costa, p. 125, pl. 11, fig. 11.

1862 Amphorina gracillima Seguenza, p. 51, pl. 1, fig. 37.

1913 Lagena gracilima (Seguenza) - Cushman, p. 11, pl. 1, fig. 4.

1956 Lagena gracilima (Seguenza) - Asano (a), p. 30, pl. 5, figs. 11-13.

1990 Lagena gracilima (Seguenza) - Ujiié, pp. 17, 18, pl. 4, fig. 14.

1994 Procerolagena gracilima (Seguenza) Jones, p. 62, pl. 56, figs. 19-22, 24-29.

2002 Hyalinonetrion gracilima (Seguenza) Akimoto, Matsui, Shimokawa, and Furukawa, p. 10, 11, pl. 23, fig. 5.

2012 Hyalinonetrion gracillium (Costa) - Milker and Schmiedl, p. 74, fig. 18.30.

Remarks. We basically follow Milker and Schmiedl (2012) for synonymy. Loeblich and Tappan (1994) distinguished specimens of Lagena gracilima Seguenza of Brady's (1884) "Challenger Report" (pl. 56 , figs. 25,26 ) as Hyalinonetrion dissomapolitium, but here we accept a wider range of variation in test inflation.

Occurrence. Rare in the Yonahama and Minebari formations.

Genus LAGENA Walker and Jacob in Kanmacher, 1798

Lagena sulcata (Walker and Jacob)

Figures 13.10, 13.11

1798 Serpula (Lagena) sulcata Walker and Jacob in Kanmacher, p. 634, pl. 14, fig. 5.

1944 Lagena sulcata (Walker and Jacob) LeRoy (Part 1), p. 23, fig. 9.
1987 Lagena sulcata (Walker and Jacob) Loeblich and Tappan, pl. 455, figs. 12, 13.

1994 Lagena sulcata (Walker and Jacob) Jones, p. 64 , pl. 57 , figs. 23, 25-27, 33, 34.

Occurrence. Rare in the Onogoshi Formation, common in the Yonahama Formation.

Genus PROCEROLAGENA Puri, 1954

Procerolagena gracilis (Williamson)

Figure 13.12

1848 Lagena gracilis Williamson, p. 13.

1901 Lagena gracilis Williamson - Millett (part XI), pp. 492, 493, pl. 8, fig. 13 (non 12, 14).

1987 Procerolagena gracilis (Williamson) Loeblich and Tappan, pl. 455, fig. 2.

1994 Procerolagena gracilis (Williamson) Jones, p. 65, pl. 58, figs. 9, 11-15.

2002 Procerolagena gracilis (Williamson) Akimoto, Matsui, Shimokawa, and Furukawa, p. 11, pl. 27, fig. 2.

Remarks. We identify a small specimen with numerous, yet weak longitudinal striations as $P$. gracilis accepting a wide range of variation in the development and number of striae.

Occurrence. A single specimen from the Yonahama Formation.

Procerolagena multilatera (McCulloch)

Figures 13.13, 13.14

1933 Lagena gracilis Williamson - Cushman (c), p. 33, pl. 8, figs. 5-7 (non Williamson, 1848).

1938 Lagena gracilis Williamson - Asano (a), p. 216, pl. 27, fig. 45, pl. 30, fig. 21.

1956 Lagena gracilis Williamson - Asano (a), pp. 39, 40, pl. 5, figs. 18-20.

1977 Lagena multilatera McCulloch, pp. 40, 41, pl. 50, fig. 5.

1994 Lagena multilatera McCulloch - Jones, p. 65, pl. 58, figs. 2, 3, 7, 8, 22-24.

1994 Procerolagena multilatera (McCulloch) Loeblich and Tappan, pp. 79, 80, pl. 144, fig. 8.

Remarks. Asano (1956a) referred this species to Brady's (1884) L. gracilis, which was later identified by Jones (1994) as L. multilatera. Lagena gracilis Williamson has finer and more numerous striae than P. multilatera.

Occurrence. Rare in the Yonahama Formation.

Genus PYGMAEOSEISTRON Patterson and Richardson, 1988 


\section{Pygmaeoseistron aspera (Reuss)}

Figures $13.15,13.16$

1862Lagena aspera Reuss (a), p. 305, pl. 1, fig. 5. 1913Lagena aspera Reuss - Cushman, p. 16, pl. 16, fig. 1.

1964Lagena aspera Reuss - LeRoy, p. F25, pl. 13, fig. 29.

Remarks. Genus Pygmaeoseistron is distinguished from Lagena in lack of longitudinal striae or costae and narrow elongate neck.

Occurrence. Rare in the Onogoshi and Yonahama formations.

Pygmaeoseistron hispidulum (Cushman)

Figures 13.17, 13.18

1913 Lagena hispidula Cushman, p. 14, pl. 5, figs. 2, 3.

1987 Pygmaeoseistron hispidulum (Cushman) - Loeblich and Tappan, pl. 455, figs. 35.

1988 Lagena hispidula Cushman - Wang, Zhang, Zhao, Min, Bian, Zheng, Cheng, and Chen, p. 135, pl. 16, fig. 13.

1989 Lagena hispidula Cushman - Ōki, p. 95, pl. 6, fig. 10; Hermelin, p. 42, pl. 4, fig. 11.

1990 Lagena hispidula Cushman - Ujiié, p. 18, pl. 5, fig. 3.

1994 Lagena hispidula Cushman - Jones, p. 62, pl. 56, figs. 10, 11, 13.

Occurrence. Sporadic in the Onogoshi, Yonahama, and Minebari formations.

Pygmaeoseistron oceanicum (Albani)

Figures 13.19-13.22

1974 Lagena oceanica Albani, p. 37, pl. 1, figs. 7, 10, 11.

1989 Lagena setigera Millett — Ōki, p. 95, pl. 6, fig. 11 (non Lagena clavata var. setigera Millett, 1901, part XI).

1992 Lagena setigera Millett - Ōki and Yamamoto, p. 193, figs. 4.5.

1994 Pygmaeoseistron oceanicum (Albani) Loeblich and Tappan, p. 80, pl. 144, figs. 4-7.

Occurrence. Sporadic in the Yonahama and Minebari formations.

Family POLYMORPHINIDAE d'Orbigny, 1839a

Subfamily POLYMORPHININAE d'Orbigny, 1839a Genus SIGMOIDELLA Cushman and Ozawa, 1928
Sigmoidella elegantissima (Parker and Jones)

Figures 13.24-13.26

1865 Polymorphina elegantissima Parker and Jones, p. 438 (nomen nudum).

1871 Polymorphina elegantissima Parker and Jones in Brady, Parker, and Jones, p. 231, pl. 40, fig. 15.

1913 Polymorphina elegantissima Parker and Jones - Cushman, p. 90, pl. 38, fig. 1.

1921 Polymorphina elegantissima Parker and Jones - Cushman, pp. 267, 268, pl. 54, figs. 1, 2.

1937 Sigmoidella subtaiwanensis Nakamura, p. 138, pl. 11, fig. 6.

1994 Sigmoidella elegantissima (Parker and Jones) - Jones, p. 84, pl. 72, figs. 1215; Loeblich and Tappan, p. 83, pl. 148, figs. 4-12.

Occurrence. Common in the Oura Formation, a single specimen from the Yonahama Formation.

Family ELLIPSOLAGENIDAE Silvestri, 1923

Subfamily OOLININAE Loeblich and Tappan, 1961

Genus EXSCULPTINA Patterson and Richardson, 1988

Exsculptina glaphyraheda Loeblich and Tappan

Figure 13.27

1901 Lagena crenata Parker and Jones - Millett (Part XI), p. 485, pl. 8, fig. 1 (non Parker and Jones, 1865).

1994 Lagena crenata Parker and Jones Jones, p. 64, pl. 57, fig. 15.

1994 Exsculptina glaphyraheda Loeblich and Tappan, p. 85, pl. 151, figs. 5-10.

Remarks. As described by Loeblich and Tappan (1994), this species differs from $L$. crenata in ornament of the basal part and the annulated apertural neck.

Occurrence. A single specimen from the Yonahama Formation.

Genus FAVULINA Patterson and Richardson, 1987 Favulina melo (d'Orbigny)

Figure 13.28

1839 Oolina melo d'Orbigny (c), p. 20, pl. 5, fig. 9.

1988 Oolina melo d'Orbigny - Wang, Zhang, Zhao, Min, Bian, Zheng, Cheng, and Chen, p. 143 , pl. 19, fig. 2.

1989 Oolina melo d'Orbigny - Ōki, pp. 101, 102, pl. 7, fig. 11. 
2002 Favulina melo (d'Orbigny) - Akimoto, Matsui, Shimokawa, and Furukawa, p. 12, pl. 30, fig. 2.

Occurrence. A single specimen from the Minebari Formation.

\section{Favulina scalariformis (Williamson)}

Figure 14.1

1858 Entosolenia squamosa var. scalariformis Williamson, p. 13, pl. 1, fig. 30.

1913 Lagena hexagona var. scalariformis (Williamson) - Cushman, p. 17, pl. 6, fig. 4.

1964 Oolina squamosa var. scalariformis (Williamson) - LeRoy, p. F26, pl. 13, fig. 43.

1977 Oolina scalariformis (Williamson) McCulloch, p. 84, pl. 54, fig. 20.

2001 Favulina scalariformis (Williamson) Kawagata, p. 79, fig. 6-7.

2002 Favulina scalariformis (Williamson) Akimoto, Matsui, Shimokawa, and Furukawa, p. 12, pl. 29, fig. 3.

Occurrence. A single specimen from the Minebari Formation.

Subfamily ELLIPSOLAGENINAE Silvestri, 1923 Genus FISSURINA Reuss, 1850

Fissurina angulata (Uchio)

Figures 14.2, 14.3

1951 Entosolenia marginata var. angulata Uchio, p. 38, pl. 3, fig. 14.

Diagnosis. A small species with thick peripheral keel which lends it a near-rectangular cross section.

Occurrence. Four specimens from a single sample of the Yonahama Formation.

\section{Fissurina bradii Silvestri}

Figures 14.4, 14.5

1902 Fissurina bradii Silvestri, p. 147.

1992 Fissurina bradii Silvestri - Kaiho, pl. 2, fig. 11.

1994 Fissurina bradii Silvestri - Jones, p. 68, pl. 59, fig. 24.

Occurrence. Rare in the Yonahama and Minebari formations.

\section{Fissurina caribaea (Cushman)}

Figures 14.6, 14.7

1923 Lagena orbignyana var. caribaea Cushman, p. 41, pl. 7, figs. 6-9.

1968 Fissurina caribaea (Cushman) - Huang, p. 57 , pl. 13, fig. 5 .

Occurrence. Common in the Yonahama and Minebari formations.
Fissurina circularis Todd

Figures 14.8, 14.9

1954 Fissurina circularis Todd in Cushman, Todd and Post, p. 351, pl. 87, fig. 27.

1994 Fissurina circularis Todd - Loeblich and Tappan, p. 88, pl. 154, figs. 13, 14.

2001 Fissurina sp. D - Kawagata, p. 84, figs. 79, 7-10.

Occurrence. Sporadic in the Onogoshi, Yonahama, and Minebari formations.

Fissurina marginata (Montagu)

Figures 14.10, 14.11

1803 Vermiculum marginatum Montagu, p. 524.

1913 Lagena marginata (Montagu) - Cushman, pp. 37,38 , pl. 22, figs. $1-7$.

1956 Fissurina marginata (Montagu) - Asano (a), pp. 53, 54, pl. 5, figs. 59, 60 .

1959 Fissurina marginata (Montagu) - Graham and Militante, p. 82, pl. 12, fig. 24.

2001 Fissurina marginata (Montagu) Kawagata, p. 81, fig. 7-3.

Occurrence. Sporadic in the Onogoshi and Yonahama formations.

\section{Fissurina quadrata (Williamson)}

Figure 14.12

1858 Entosolenia marginata var. quadrata Williamson, p. 11, pl. 1, figs. 27, 28.

1994 Fissurina quadrata (Williamson) - Loeblich and Tappan, p. 90, pl. 155, figs. $1-6$.

Occurrence. A single specimen from the Minebari Formation.

Subfamily PARAFISSURININAE Jones, 1984

Genus PARAFISSURINA Parr, 1947

Parafissurina basispinata McCulloch

Figure 14.13

1977 Parafissurina basispinata McCulloch, p. 139, pl. 72, figs. 1-3.

1994 Parafissurina basispinata McCulloch Loeblich and Tappan, p. 94, pl. 162, figs. $1-5$.

Occurrence. A single specimen from the Yonahama Formation.

\section{Parafissurina lata (Wiesner)}

Figures $14.14,14.15$

1931 Ellipsolagena lata Wiesner, p. 126, pl. 24, figs. K, L.

1990 Parafissurina lata (Wiesner) - Ujiié, pp. 27, 28, pl. 10, figs. 5-7. 


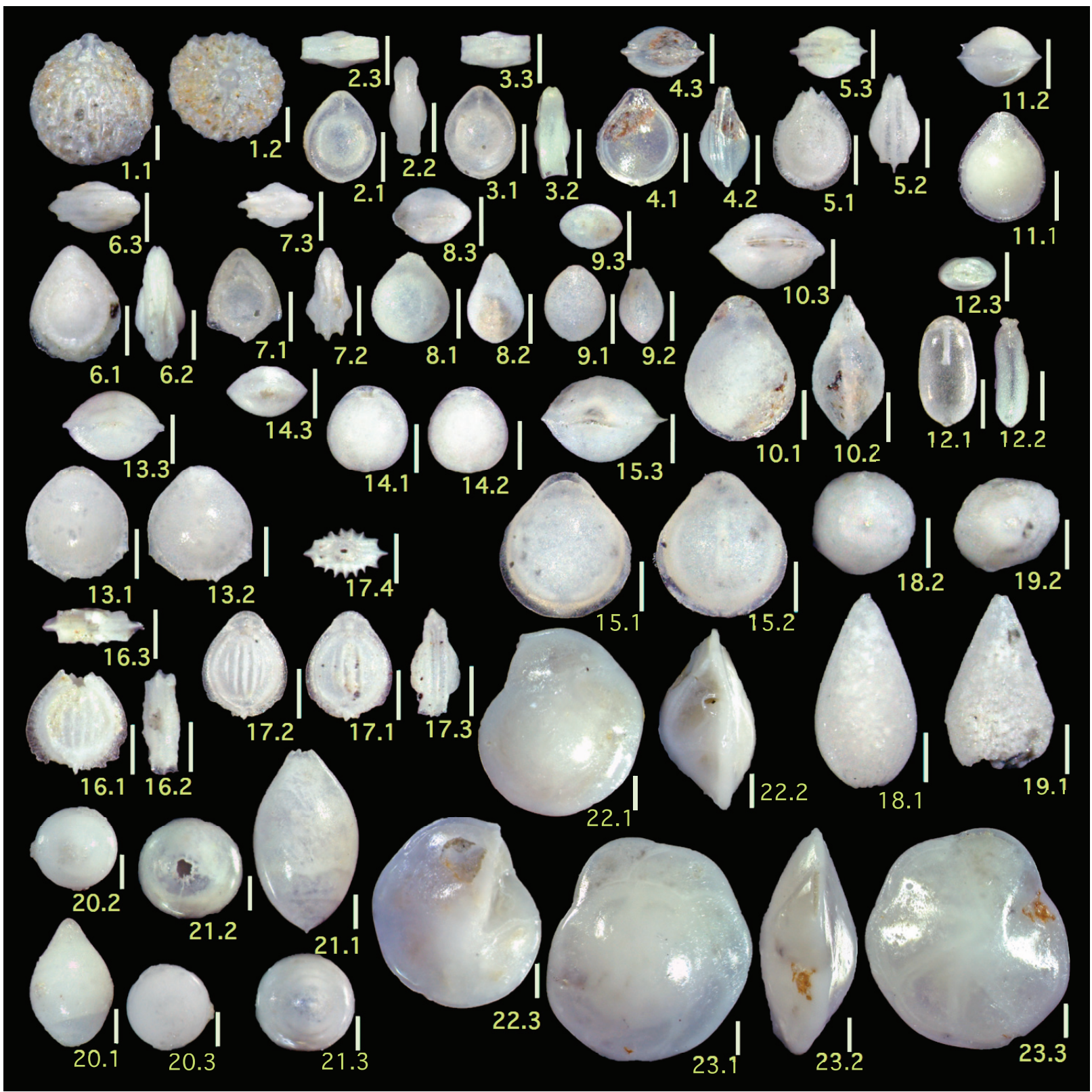

FIGURE 14. Family Ellipsolagenidae, Glandulinidae, and Epistominidae. All scale bars $=0.1 \mathrm{~mm}$. 1. Favulina scalariformis (Williamson), MPC-26258 from sample 4 (Minebari Formation, PL5). 2, 3. Fissurina angulata (Uchio), 2: MPC26261, 3: MPC-26262, both from sample MK01 (Yonahama F., PL2). 4, 5. Fissurina bradii Silvestri, 4: MPC-26263 from sample 6 (Yonahama F., PL2); 5: MPC-26264 from sample 14-2 (Yonahama F., PL4). 6, 7. Fissurina caribaea (Cushman), 6: MPC-26265 from sample 15 (Yonahama F., PL3); 7: MPC-26266 from sample MK01 (Yonahama F., PL2). 8, 9. Fissurina circularis Todd, 8: MPC-26267 from sample 3-2-low-silt (Onogoshi F., PL1); 9: MPC-26268 from sample MK02B (Minebari F., PL5). 10, 11. Fissurina marginata (Montagu), 10: MPC-26269 from sample 3-2-low-silt (Onogoshi F., PL1); 11: MPC-26270 from sample 16 (Yonahama F., PL3). 12. Fissurina quadrata (Williamson), MPC26271 from sample 1 (Minebari F., PL5). 13. Parafissurina basispinata McCulloch, MPC-26404 from sample 7 (Yonahama F., PL4). 14, 15. Parafissurina lata (Wiesner), 14: MPC-26405 from sample 2 (Minebari F., PL5); 15: MPC26406 from sample MK01 (Yonahama F., PL2). 16, 17. Pseudofissurina metaconica (McCulloch), 16: MPC-26432 from sample 8-2 (Yonahama F., PL4); 17: MPC-26433 from sample 17 (Yonahama F., PL2). 18, 19. Montcharmontzeiana leminiscata (Loeblich and Tappan), 18: MPC-26377, 19: MPC-26378, both from sample 7 (Yonahama F., PL4). 20, 21. Glandulina ovula d'Orbigny, 20: MPC-26279 from sample 7 (Yonahama F., PL4); 21: MPC-26280 from sample 10 (Onogoshi F., PL2). 22, 23. Hoeglundina elegans (d'Orbigny), 22: MPC-26322 from sample 4 (Minebari F., PL5); 23: MPC-26323 from sample 13 (Minebari F., PL5). 
1995 Parafissurina lata (Wiesner) - Ujiié, p. 59, pl. 88, fig. 10.

Occurrence. Rare in the Yonahama and Minebari formations.

Genus PSEUDOFISSURINA Jones, 1984

Pseudofissurina metaconica (McCulloch)

Figures 14.16, 14.17

1977 Parafissurina metaconica McCulloch, pp. 150, 151, pl. 71, fig. 24.

1990 Parafissurina acuticostata Ujiié, p. 88, pl. 11, figs. 1, 2.

1994 Pseudofissurina metaconica (McCulloch) - Loeblich and Tappan, p. 95, pl. 166, figs. $1-5$.

Occurrence. Sporadic in the Yonahama Formation.

Subfamily SIPHOLAGENINAE Patterson and Richardson, 1987

Genus MONCHARMONTZEIANA Patterson, 2010

Moncharmontzeiana leminiscata (Loeblich and Tappan)

Figures 14.18, 14.19

1994 Pytine laminiscata Loeblich and Tappan, $\mathrm{p}$. 96, pl. 140, figs. $1-5$.

Remarks. Patterson (2010) proposed a new genus to replace a junior homonym, Pytine (Moncharmont Zei and Sgarrella, 1978). Pyriform test with very fine mesh ornamentation on surface characterizes this genus.

Occurrence. Two specimens from a single sample of the Yonahama Formation.

Family GLANDULINIDAE Reuss, 1860

Subfamily GLANDULININAE Reuss, 1860

Genus GLANDULINA d'Orbigny, 1839a Glandulina ovula d'Orbigny

Figures 14.20, 14.21

1846 Glandulina ovula d'Orbigny, p. 29, pl. 1, figs. $6,7$.

1964 Rectoglandulina laevigata (d'Orbigny) LeRoy, p. F23, pl. 14, figs. 29, 30 (non Glandulina laevigata d'Orbigny, 1826).

1977 Euglandulina symmetrica McCulloch, p. 14, pl. 96, fig. 11.

1985 Glandulina ovula d'Orbigny - Papp and Schmid, pp. 21, 22, pl. 2, figs. 1-9.

1994 Glandulina ovula d'Orbigny - Jones, p. 72, pl. 61, figs. $17-22$, pl. 63, fig. 6 .

1994 Glandulina symmetrica (McCulloch) Loeblich and Tappan, p. 97, pl. 168, figs. 6 -8.
Remarks. The microspheric form occasionally shows a small apiculate projection, whereas the megalospheric form shows a rounded proloculus. It is distinguished from $G$. laevigata in having a more inflated, fusiform test.

Occurrence. Rare in the Onogoshi, Yonahama, and Minebari formations.

Suborder ROBERTININA Loeblich and Tappan, 1984

Superfamily CERATOBULIMINOIDEA Cushman, $1927 a$

Family EPISTOMINIDAE Wedekind, 1937

Subfamily EPISTOMININAE Wedekind, 1937

Genus HOEGLUNDINA Brotzen, 1948 Hoeglundina elegans (d'Orbigny)

Figures 14.22-15.2

1826 Rotalia (Turbinulina) elegans d'Orbigny, p. 276.

1941 Epistomina elegans (d'Orbingy) - LeRoy (Part 1), pp. 40, 41, pl. 1, figs. 5-7; LeRoy (Part 2), p. 84, pl. 4, figs. 13-15.

1944 Epistomina elegans (d'Orbingy) - LeRoy (Part 1), pp. 35, 36, pl. 3, figs. 15-17.

1964 Hoeglundina elegans (d'Orbigny) LeRoy, p. F38, pl. 6, figs. 27, 28.

1965 Hoeglundina elegans (d'Orbigny) - Todd, pp. 56, 57, pl. 23, fig. 2.

1966 Hoeglundina elegans (d'Orbigny) - Belford, pp. 190, 191, pl. 36, figs. 8-13.

1985 Hoeglundina elegans (d'Orbigny) - Papp and Schmid, p. 59, pl. 49, figs. $1-6$.

1987 Hoeglundina elegans (d'Orbigny) - Loeblich and Tappan, pl. 487, figs. 1-5.

1988 Hoeglundina elegans (d'Orbigny) Wang, Zhang, Zhao, Min, Bian, Zheng, Cheng, and Chen, p. 145, pl. 20, figs. 24; Marle, p. 145, pl. 5, figs. 18, 19.

1989 Hoeglundina elegans (d'Orbigny) - Ōki, pp. 153, 154, pl. 22, fig. 7; Inoue, pl. 21, fig. 5 , pl. 31 , fig. 13; Hess, p. 83 , pl. 14 , figs. $7,8$.

1990 Hoeglundina elegans (d'Orbigny) Akimoto, p. 202, pl. 21, fig. 7, pl. 24, fig. 6; Ujiié, p. 53, pl. 32, figs. 3, 4.

1992 Hoeglundina elegans (d'Orbigny) - Hatta and Ujiié (b), p. 170, pl. 24, fig. 3.

1994 Hoeglundina elegans (d'Orbigny) Jones, pp. 104, 105, pl. 105, figs. 3-6; Loeblich and Tappan, p. 98, pl. 174, figs. 1-6; Akimoto, p. 284, pl. 3, fig. 7. 
1998 Hoeglundina elegans (d'Orbigny) - Hess, p. 83 , pl. 14 , figs. 7,8 .

Remarks. Most of the specimens exhibit eight chambers in the final whorl, whereas four relatively large individuals from the Minebari Formation show up to 14 chambers in the ultimate whorl. To the best of our knowledge, this large multichambered form has not been illustrated in previous work.

Occurrence. Rare in the Yonahama Formation, common in the Minebari Formation.

\section{Suborder GLOBIGERININA Delage and Hérouard, 1896 \\ Superfamily GLOBOROTALIOIDEA Cushman, $1927 a$}

Family GLOBOROTALIIDAE Cushman, 1927a

Genus CLAVATORELLA Blow, 1965

Clavatorella aff. suturanii Giannelli and Salvatorini

Figures 15.3, 15.4

Compared with:

1976 Clavatorella suturanii Giannelli and Salvatorini, p. 168, pl. 1, fig. 1.

1985 Clavatorella suturanii Giannelli and Salvatorini - Bolli and Saunders, 1985, p. 255, fig. 45.3.

2004 Clavatorella sp. 1 - Hanagata, pl. 3, fig. 9. Diagnosis. Small species with four to four and a half, slightly elongated chambers in the final whorl, moderately perforated smooth surface without ornament, aperture small interiomarginal U-shaped opening from umbilicus to near the periphery. Outer margin of chambers is lobulate and occasionally apiculate.

Remarks. This form is distinguishable from $C$. suturanii in having less inflated and less lobulate chambers. Kadar (1975, pl. 8, fig. 59) illustrated a morphotype of Clavatorella (Clavatorella) bermudezi (Bolli) that is somewhat similar to the present form, but differs in having more elongated chambers and an umbilicus-oriented aperture. Clavatorella suturanii and $C$. bermudezi have both been recorded from the Miocene; thus the present form is probably a descendant.

Occurrence. Common in the Yonahama Formation, sporadic in the Minebari Formation.

\section{Genus GLOBOROTALIA Cushman, 1927a Globorotalia conoidea Walters}

Figures 15.5, 15.6

1965 Globorotalia miozea conoidea Walters, p. 124, fig. 8.

1974 Globorotalia aff. cibaoensis Ujiié and Ōki, pl. 5, figs. $1-4$.
1977 Globorotalia miozea conoidea Walters Berggren, p. 298, pl. 2, figs. 9-31.

1978 Globorotalia miozea conoidea Walters Keller (a), pl. 1, figs. 7-10.

1983 Globorotalia (Globoconella) conoidea Walters - Kennett and Srinivasan, p. 112, pl. 26, figs. 4-6.

1985 Globorotalia (Globorotalia) miozea conoidea Walters - Ibaraki, p. 132, pl. 10, figs. $12-14$.

1985 Globorotalia (s.s.) aff. pliozea Hornibrook — Ujiié, p. 113, pl. 14, figs. 5-7.

1985 Globorotalia conoidea Walters - Jenkins, p. 276 , figs. $7.14,7.15$.

Remarks. Specimens from Miyakojima Island exhibit a transitional form between G. conoidea and Globorotalia conomiozea. It has four chambers in the final whorl similar to $G$. conomiozea, while the umbilical side is less vaulted than typical $G$. conoidea as figured by Kennett and Srinivasan (1983). Natori (1976, table 2) showed in his range chart that G. conomiozea evolved into G. conoidea; whereas Kennett and Srinivasan (1983) noted the opposite and showed that G. conoidea disappeared in the uppermost Miocene. In Miyakojima Island, G. conomiozea disappears earlier than $G$. conoidea as noted by Natori (1976) in Okinawajima, both in the Pliocene. Based on those reports, it can be stated that $G$. conoidea and G. conomiozea are potentially useful indicators of the upper Miocene to lower Pliocene of subtropical to temperate seas, but their ranges vary between areas, and further stratigraphic studies are called for.

Occurrence. Common in the upper part of the Yonahama Formation.

\section{Globorotalia conomiozea Kennett}

Figures 15.7, 15.8

1966 Globorotalia conomiozea Kennett, p. 235, text-fig. 10.

1976 Globorotalia (Globorotalia) crassula conomiozea Kennett - Natori, p. 229, pl. 5, fig. 3.

1977 Globorotalia conomiozea Kennett - Berggren, pp. 298, 300, pl. 3, figs. 7-12.

1978 Globorotalia conomiozea Kennett - Oda, pp. 56,57 , pl. 8 , figs. 4-6; Keller (a), pl. 1, figs. $1-6$.

1983 Globorotalia (Globoconella) conomiozea Kennett - Kennett and Srinivasan, p. 114, pl. 26, figs. 7-9. 


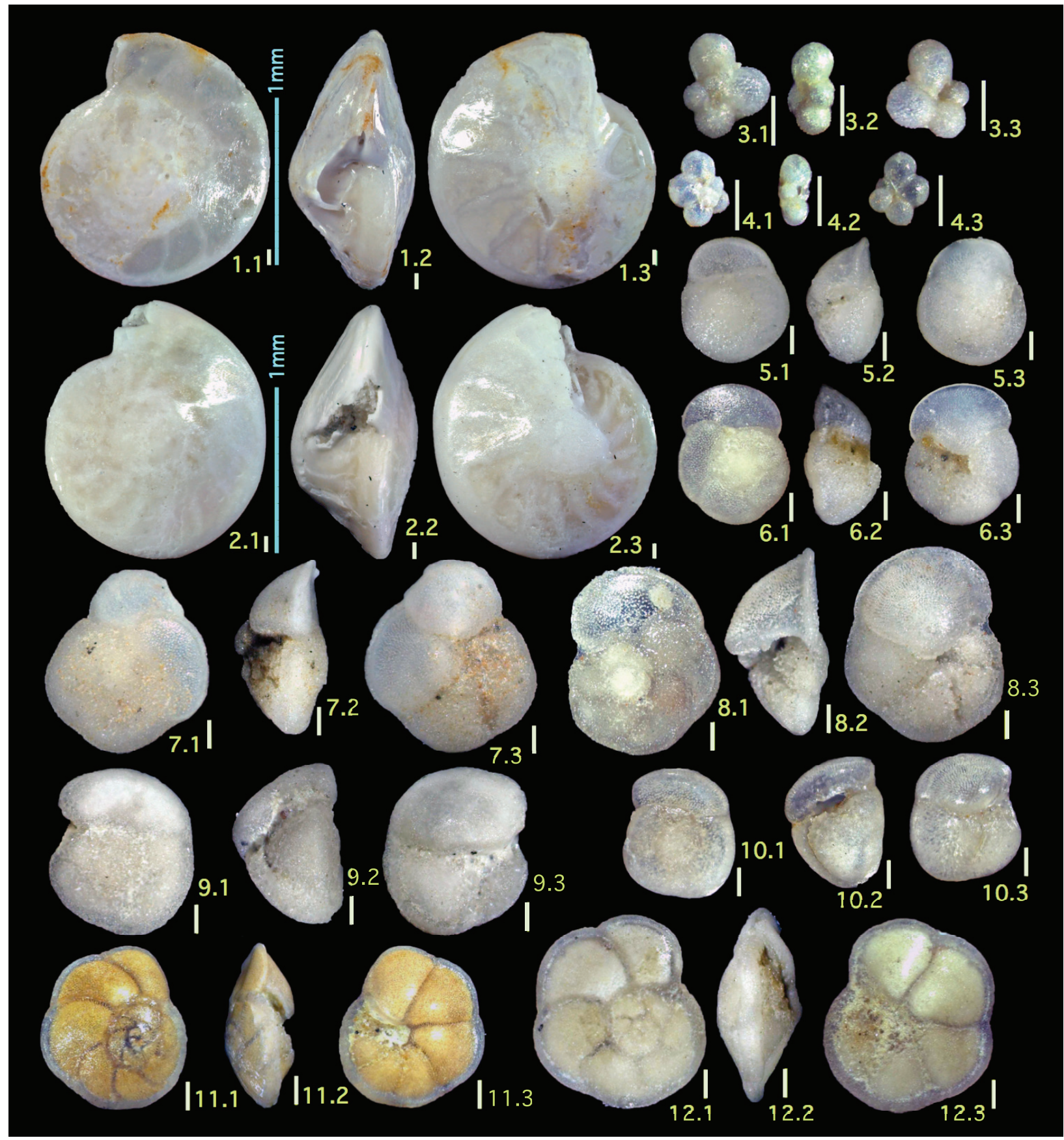

FIGURE 15. Family Epistominidae and Globorotaliidae. All scale bars $=0.1 \mathrm{~mm}$, unless otherwise indicated. $\mathbf{1}, \mathbf{2}$. Hoeglundina elegans (d'Orbigny) large form, 1: MPC-26324 from sample 2-2 (Minebari Formation, PL5); 2: MPC26325 from sample 4 (Minebari F., PL5). 3, 4. Clavatorella aff. suturanii Giannelli and Salvatorini, 3: MPC-25930 from sample 5 (Minebari F., PL5); 4: MPC-25931 from sample MK01 (Yonahama F., PL2). 5, 6. Globorotalia conoidea Walters, 5: MPC-25968 from sample 7 (Yonahama F., PL4); 6: MPC-25969 from sample 8-2 (Yonahama F., PL4). 7, 8. Globorotalia conomiozea Kennett, 7: MPC-25970 from sample 3 (Onogoshi F., PL1); 8: MPC-25971 from sample 6 (Yonahama F., PL2). 9, 10. Globorotalia crassaformis (Galloway and Wissler), 9: MPC-25972 from sample MK02A (Minebari F., PL5); 10: MPC-25973 from sample MK02B (Minebari F., PL5). 11, 12. Globorotalia cultrata (d'Orbigny), 11: MPC-25974 from sample MK01 (Yonahama F., PL2); 12: MPC-25975 from sample MK02A (Minebari F., PL5). 
1985 Globorotalia conomiozea Kennett - Jenkins, pp. 276, 278, figs. 7, 18.

1985 Globorotalia (s.s.) sp. A - Ujiié, p. 114, pl. 17, fig. 1.

1985 Globorotalia (Globorotalia) conomiozea Kennett - Ibaraki, p. 129, pl. 9, figs. 1, 2.

Occurrence. Common in the Onogoshi Formation and the lower part of the Yonahama Formation.

Globorotalia crassaformis (Galloway and Wissler) Figures 15.9, 15.10

1927 Globigerina crassaformis Galloway and Wissler (a), p. 41, pl. 7, fig. 12.

1921 Pulvinulina crassa (d'Orbigny) - Cushman, p. 338, pl. 67, fig. 3.

1962 Globorotalia crassaformis (Galloway and Wissler) - Parker, p. 235, pl. 4, figs. 17, 18, 20, 21.

1967 Globorotalia crassaformis (Galloway and Wissler) - Parker, p. 176, pl. 30, figs. 13.

1968 Globorotalia crassaformis (Galloway and Wissler) - Huang, pl. 61, pl. 11, figs. 7, 11.

1969 Globorotalia (Truncorotalia) crassaformis (Galloway and Wissler) - Blow, p. 347, pl. 4 , figs. $1-3$, pl. 37 , figs. $1-4$.

1974 Globorotalia (Turborotalia) crassaformis (Galloway and Wissler) - Ujiié and Ōki, pl. 4, figs. 1, 2.

1975 Globorotalia (Turborotalia) crassaformis crassaformis (Galloway and Wissler) Ibaraki and Tsuchi, pl. 3, fig. 9.

1976 Globorotalia (Turborotalia) crassaformis crassaformis (Galloway and Wissler) Natori, p. 229, pl. 3, fig. 2.

1978 Globorotalia crassaformis (Galloway and Wissler) - Oda, p. 57, pl. 9, figs. 1-3.

1977 Globorotalia crassaformis (Galloway and Wissler) - Kadar, p. 68, pl. 7, fig. 38.

1978 Globorotalia crassaformis crassaformis (Galloway and Wissler) - Keller (a), pl. 2, figs. 5-12.

1981 Globorotalia crassaformis (Galloway and Wissler) - Saito, Thompson, and Breger, pp. 129, 130, pl. 43, fig. 2.

1983 Globorotalia (Truncorotalia) crassaformis (Galloway and Wissler) - Kennett and Srinivasan, p. 146, pl. 34, figs. 6-8.

1985 Globorotalia crassaformis crassaformis (Galloway and Wissler) - Bolli and Saunders, p. 233, figs. 36.6, 36.7.
1985 Globorotalia (Turborotalia) crassaformis crassaformis (Galloway and Wissler) Ibaraki, pp. 123, 124, pl. 7, figs. 1, 2.

1985 Globorotalia (Turborotalia) crassaformis (Galloway and Wissler) - Ujiié, p. 112, pl. 12, figs. 2-4, 8, 9, pl. 13, figs. 1, 6 .

1994 Globorotalia (Truncorotalia) crassaformis (Galloway and Wissler) - Jones, p. 103, pl. 103, figs. 11, 12.

1994 Truncorotalia crassaformis (Galloway and Wissler) - Loeblich and Tappan, p. 102, pl. 186, figs. $1-9$.

2004 Truncorotalia crassaformis (Galloway and Wissler) - Hanagata, pl. 3, fig. 2.

Remarks. Although, in a previous report (Hanagata, 2004), the generic name Truncorotalia was applied to plano-convex globorotaliids, following the taxonomy of Loeblich and Tappan (1987), we here assign those species to Globorotalia. The phylogenetic relationship between two clades of plano-convex planktonic foraminiferal groups is still unclear; i.e., Globorotalia miozea - conoidea conomiozea - puncticulata - inflata lineage and Globorotalia cibaoensis - juanai - crassula crassaformis - tosaensis - truncatulinoides lineage. These are assumed to have evolved independently from the same species, Globorotalia praescitula (Norris et al., 1994). A study of the Miocene differentiation from $G$. praescitula will resolve this problem.

Occurrence. Common in the upper part of the Yonahama Formation up to the Minebari Formation.

\section{Globorotalia cultrata (d'Orbigny)}

Figures 15.11, 15.12

1921 Pulvinulina menardii (d'Orbigny) - Cushman, pp. 333, 334, pl. 66, fig. 1. (non d'Orbigny, 1839a).

1941 Globorotalia menardii (d'Orbigny) LeRoy (Part 1), p. 45, pl. 1, figs. 17-19; LeRoy (Part 2), p. 87, pl. 4, figs. 4-6; LeRoy (Part 3), pp. 118, 119, pl. 1, figs. 68.

1944 Globorotalia menardii (d'Orbigny) LeRoy (Part 1), p. 41, pl. 2, figs. 40-42.

1959 Globorotalia cultrata (d'Orbigny) - Wallers and Polski, pl. 10, fig. 3.

1959 Globorotalia menardii (d'Orbigny) - Graham and Militante, p. 114, pl. 19, fig. 6. 
1962 Globorotalia cultrata (d'Orbigny) - Parker, pp. 235, 236, pl. 5, figs. 3-5; Belford, p. 24 , pl. 6 , figs. $18-22$.

1964 Globorotalia menardii multicamerata Cushman and Jarvis - LeRoy, p. F43, pl. 9, figs. 16, 17 (non Cushman and Jarvis, 1930).

1967 Globorotalia cultrata (d'Orbigny) - Parker, pp. 177, 178, pl. 31, figs. 2, 3.

1969 Globorotalia (Globorotalia) cultrata cultrata (d'Orbigny) - Blow, p. 358, pl. 6, figs. 4-8.

1976 Globorotalia (Globorotalia) cultrata menardii (Parker, Jones, and Brady) - Natori, p. 229, pl. 4, fig. 6.

1977 Globorotalia cf. menardii (d'Orbigny) McCulloch, p. 419, pl. 177, fig. 1 (non p. 420 , pl. 177, fig. 2).

1977 Globorotalia cultrata (d'Orbigny) - Kadar, p. 68 , pl. 7, fig. 40.

1977 Globorotalia menardii (d'Orbigny) Kadar, p. 69, pl. 6, fig. 32, pl. 5, figs. 31 , 32.

1985 Globorotalia cultrata cultrata (d'Orbigny) Ibaraki, p. 130, pl. 9, figs. 7, 8.

1985 Globorotalia menardii cultrata (d'Orbigny) - Bolli and Saunders, p. 226, figs. 32.3, 34.8-34.10.

1985 Globorotalia cultrata (d'Orbigny) - Ujiié, pl. 15, fig. 6.

1988 Globorotalia menardii menardii - Wang, Zhang, Zhao, Min, Bian, Zheng, Cheng, and Chen, p. 47, pl. 2, figs. ?14, 15-17.

1994 Globorotalia menardii (Parker, Jones, and Brady) - Loeblich and Tappan, p. 101, pl. 183, figs. 6-6.

2004 Globorotalia menardii (Parker, Jones, and Brady) - Hanagata, pl. 2, figs. 1-6.

Remarks. Specimens from Miyakojima Island have five to six, occasionally seven, chambers in the final whorl, and peripheral keels are not robust; as such they are distinguishable from G. menardii (Parker, Jones, and Brady). Coiling directions are mixed, but the dextral form predominates except for a single sample, MK02B. This trend in coiling direction is in accordance with those recorded for the Pliocene of other regions (see summary in Bolli and Saunders, 1985).

Occurrence. Common to abundant in all the formations of the present study.
Globorotalia margaritae Bolli and Bermúdez

Figures 16.1, 16.2

1965 Globorotalia margaritae Bolli and Bermúdez, p. 132, pl. 1, figs. 16-18.

1967 Globorotalia margaritae Bolli and Bermúdez - Parker, pp. 179, 180, pl. 32, figs. 1, 2.

1969 Globorotalia margaritae Bolli and Bermudez - Blow, p. 363, pl. 45, figs. 1-3, 5, 6, pl. 44 , figs. 4,5 .

1974 Globorotalia margaritae Bolli and Bermudez, var. - Ujiié and Ōki, pl. 6, fig. 1.

1976 Globorotalia margaritae Bolli and Bermúdez - Natori, p. 229, pl. 5, fig. 1.

1977 Globorotalia margaritae Bolli and Bermudez - Kadar, p. 68, pl. 7, fig. 39.

1978 Globorotalia margaritae Bolli and Bermudez - Bolli and Bermudez, pp. 139-141, pl. figs. 1-9; Oda, p. 58, pl. 8, figs. 1, 2; Keller (a), pl. 4, figs. 5-7.

1983 Globorotalia (Hirsutella) margaritae Bolli and Bermudez - Kennett and Srinivasan, p. 136 , pl. 32, figs. $4-6$.

1985 Globorotalia margaritae Bolli and Bermudez - lbaraki, p. 131, pl. 10, figs. 5-7.

1985 Globorotalia margaritae margaritae Bolli and Bermudez - Bolli and Saunders, p. 217, figs. 30.9-30.14.

1985 Globorotalia (s.s.) margaritae Bolli and Bermudez - Ujiié, p. 113, pl. 15, figs. 2, 3.

Occurrence. Sporadic in the Onogoshi Formation and the lower part of the Yonahama Formation.

Globorotalia oceanica Cushman and Bermúdez

Figures 16.3, 16.4

1949 Globorotalia (Turborotalia) oceanica Cushman and Bermúdez, p. 43, pl. 8, figs. 1315.

1969 Globorotalia (Turborotalia) crassaformis oceanica Cushman and Bermudez Blow, p. 348, pl. 4, figs. 7-9.

1976 Globorotalia (Turborotalia) crassaformis oceanica Cushman and Bermudez Natori, p. 229, pl. 3, fig. 1.

1978 Globorotalia crassaformis oceanica Cushman and Bermudez - Keller (a), pl. 2, figs. 1-4.

1981 Globorotalia oceanica Cushman and Bermudez - Saito, Thompson, and Breger, p. 132, pl. 44, fig. 1. 


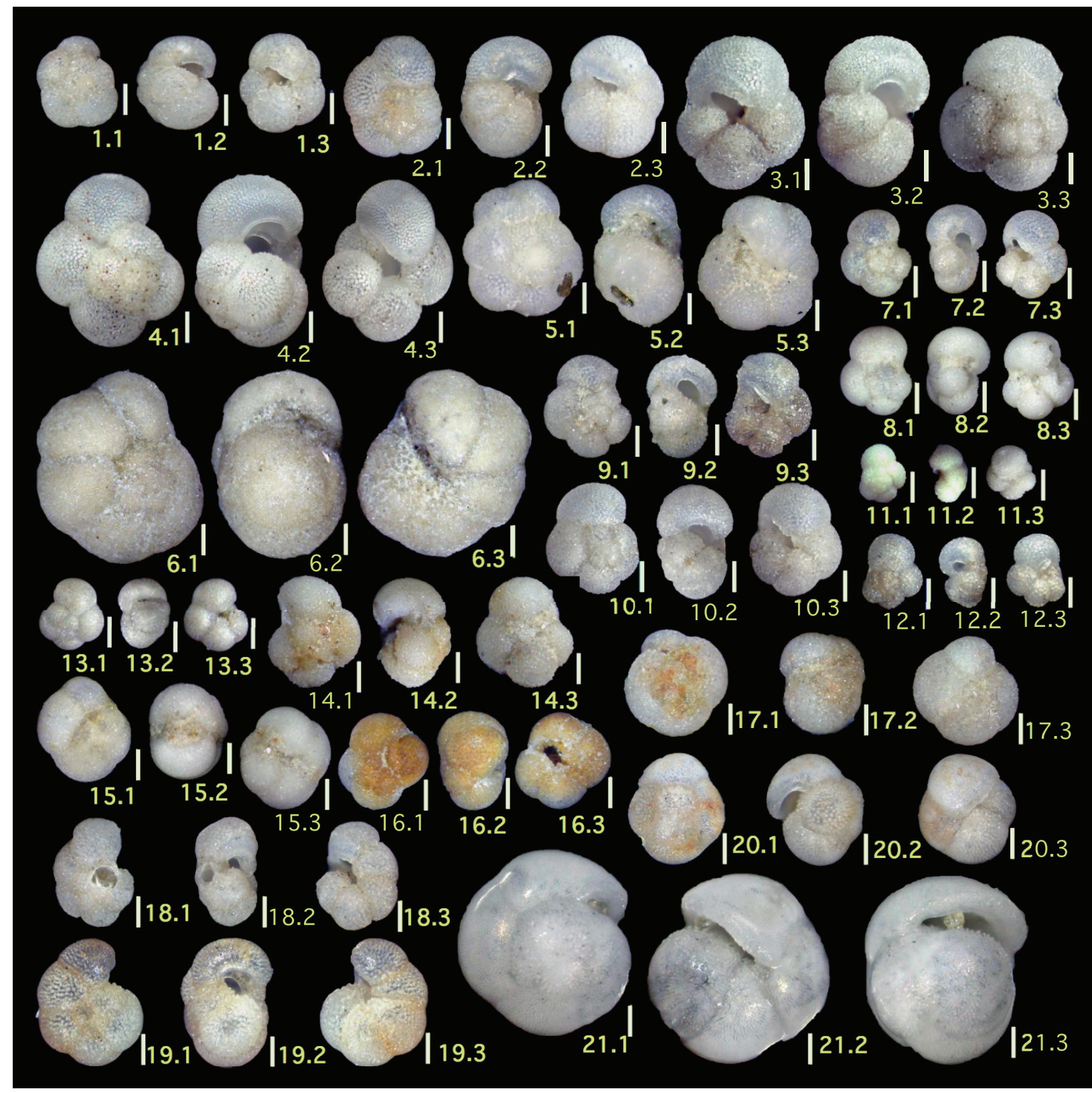

FIGURE 16. Family Globorotaliidae. All scale bars $=0.1 \mathrm{~mm}$, unless otherwise indicated. 1, 2. Globorotalia margaritae Bolli and Bermúdez, 1: MPC-25976 from sample 3 (Onogoshi Formation, PL1); 2: MPC-25977 from sample 10 (Onogoshi F., PL2). 3, 4. Globorotalia oceanica Cushman and Bermúdez, 3: MPC-25978, 4: MPC-25979, both from sample MK02A (Minebari F., PL5). 5, 6. Globorotalia scitula (Brady), 5: MPC-25980 from sample 8 (Yonahama F., PL4); 6: MPC-25981 from sample MK01 (Yonahama F., PL2). 7, 8. Globorotalia tumida (Brady), 7: MPC-25982 from sample MK01 (Yonahama F., PL2); 8: MPC-25983 from sample MK02A (Minebari F., PL5). 9, 10. Globorotalia tumida flexuosa (Koch), 9: MPC-25984 from sample 15 (Yonahama F., PL3); 10: MPC-25985 from sample 16 (Yonahama F., PL3). 11, 12. Globorotalia viola Blow, 11: MPC-25987 from sample 1 (Minebari F., PL5); 12: MPC-25986 from sample MK02A (Minebari F., PL5). 
PE Note: Erratum to Satoshi and Takami. 2015

2 February 2015

An incorrect version of Figure 16 was presented in Satoshi and Takami. 2015. Illustrated guide to Pliocene foraminifera from Miyakojima, Ryukyu Island Arc, with comments on biostratigraphy. Palaeontologia Electronica 18.1.3A: 1-140. palaeo-electronica.org/content/2015/1016-foraminifera-of-miyakojima. The following is the correct figure.

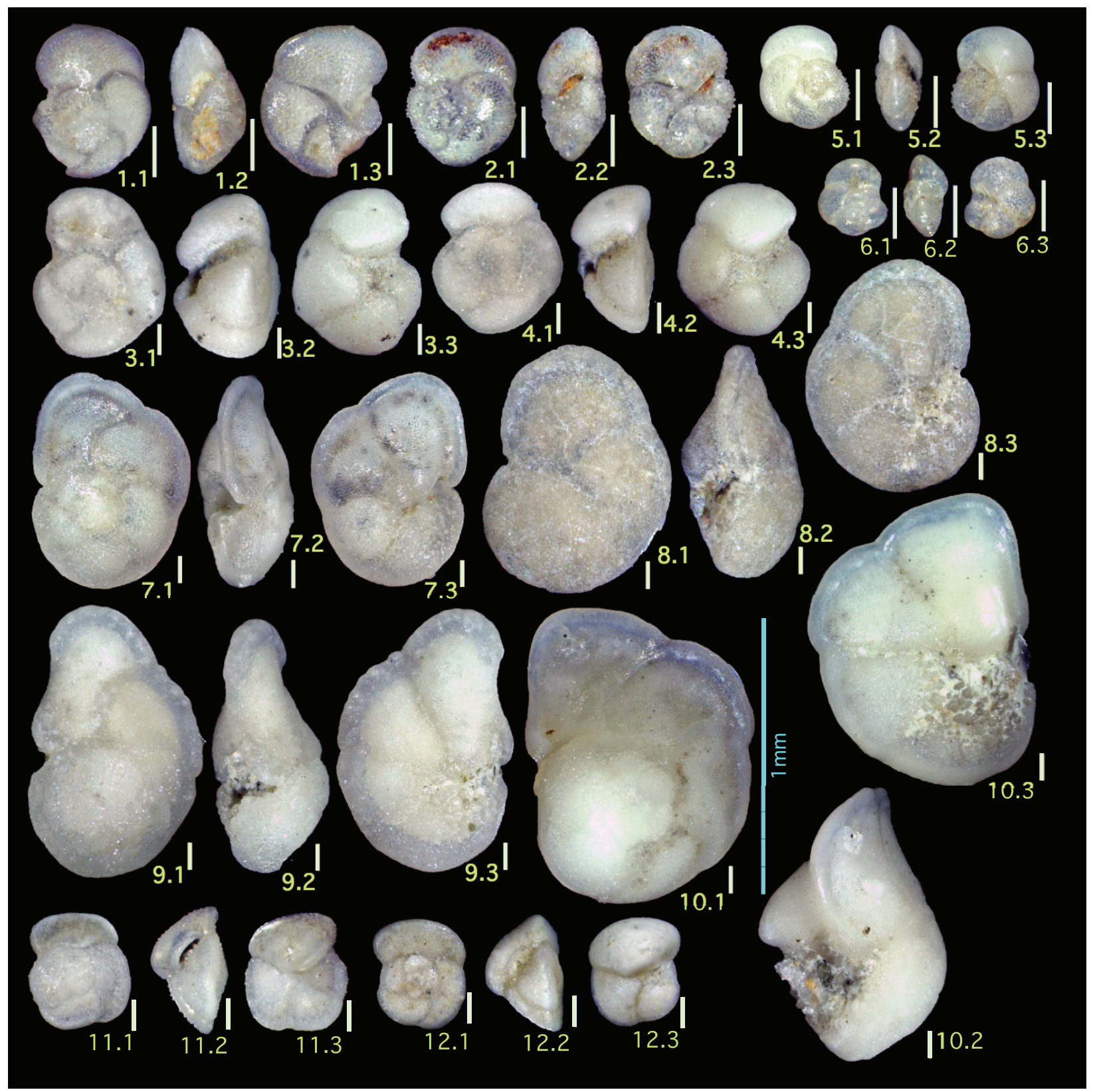


1985 Globorotalia crassaformis oceanica Cushman and Bermudez - Bolli and Saunders, p. 233, fig. 37.11.

1985 Globorotalia (Turborotalia) crassaformis oceanica Cushman and Bermudez Ibaraki, p. 124, pl. 7, figs. 3, 4.

Remarks. Differentiation of this species from Globorotalia crassaformis is difficult in young specimens that do not show such features as an open umbilicus and a wide aperture.

Occurrence. Three specimens from a single sample of the Minebari Formation.

\section{Globorotalia scitula (Brady)}

Figures 16.5, 16.6

1882 Pulvinulina scitula Brady, p. 716.

1959 Globorotalia scitula (Brady) - Bradshow, p. 44 , pl. 8 , figs. 5,6 .

1959 Globorotalia scitula scitula (Brady) Blow, pp. 219, 220, pl. 19, fig. 126.

1962 Globorotalia scitula (Brady) - Parker, pp. 238, 239, pl. 6, figs. 4-6; Belford, p. 27, pl. 7, figs. 10-13.

1967 Globorotalia scitula (Brady) - Parker, p. 181, pl. 27, fig. 7; Huang, p. 188, pl. 15, fig. 4.

1968 Globorotalia scitula (Brady) - Huang, p. 61 , pl. 10, figs. $1,6$.

1969 Globorotalia scitula (Brady) - Blow, p. 356, pl. 39, fig. 7.

1977 Globorotalia scitula (Brady) - Kadar, pl. 4, fig. 33.

1978 Globorotalia scitula (Brady) - Keller (a), pl. 4, figs. 5-12.

1978 Globorotalia scitula scitula (Brady) — Oda, p. 61, pl. 9, fig. 12.

1981 Globorotalia scitula (Brady) - Saito, Thompson, and Breger, pp. 137, 138, pl. 46, fig. 2.

1983 Globorotalia (Hirsutella) scitula (Brady) Kennett and Srinivasan, p. 134, pl. 31, figs. 1, 3-5.

1985 Globorotalia scitula scitula (Brady) - Bolli and Saunders, p. 217, figs. 30.26-30.29, 31.3, 31.4 .

1985 Globorotalia (Turborotalia) scitula scitula (Brady) - Ibaraki, p. 127, pl. 8, figs. 5, 6.

1988 Globorotalia scitula (Brady) - Wang, Zhang, Zhao, Min, Bian, Zheng, Cheng, and Chen, p. 49, pl. 4, figs. 4, 5 .
1994 Globorotalia (Obandyella) scitula (Brady) — Jones, p. 103, pl. 103, fig. 7.

1999 Globorotalia scitula (Brady) - Kim, pl. 4, figs. 7, 9.

2004 Globorotalia scitula (Brady) - Hanagata, pl. 2, fig. 3.

Occurrence. Common in the Yonahama and Minebari formations.

Globorotalia tumida (Brady)

Figures 16.7, 16.8

1877 Pulvinulina menardii (d'Orbigny) var. tumida Brady, p. 535.

1921 Pulvinulina tumida Brady - Cushman, p. 337, pl. 66, fig. 3.

1941 Globorotalia tumida (Brady) - LeRoy (Part 1), p. 45, pl. 3, figs. 101-103; LeRoy (Part 2), p. 88, pl. 4, figs. 1-3.

1959 Globorotalia tumida (Brady) - Bradshow, p. 47, pl. 8, figs. 9, 13.

1962 Globorotalia tumida (Brady) - Parker, p. 239, pl. 6, figs. 8-10; Belford, pp. 25, 26, pl. 7 , figs. $1-5$.

1964 Globorotalia tumida (Brady) - LeRoy, p. F43, pl. 9, figs. 18, 19.

1965 Globorotalia tumida (Brady) - Todd, p. 71, pl. 28, fig. 1.

1962 Globorotalia tumida (Brady) - Parker, p. 182, pl. 32, figs. 5-7.

1967 Globorotalia menardii tumida (Brady) Huang, p. 188, pl. 15, figs. 15, 16.

1968 Globorotalia tumida (Brady) - Huang, p. 61, pl. 10, fig. 19.

1969 Globorotalia (Globorotalia) tumida (Brady) - Blow, p. 371 , pl. 9, figs. 10, 11, pl. 49, figs. 1-5.

1976 Globorotalia (Globorotalia) tumida tumida (Brady) - Natori, p. 230, pl. 4, fig. 5.

1977 Globorotalia tumida (Brady) - McCulloch, p. 422 , pl. 177 , fig. 8 ; Kadar, p. 69 , pl. 7 , fig. 37, pl. 6, fig. 34, 35.

1978 Globorotalia tumida (Brady) - Keller (a), pl. 4, figs. 13-16.

1978 Globorotalia tumida tumida (Brady) Oda, p. 62, pl. 7, figs. 8-11.

1981 Globorotalia tumida (Brady) - Saito, Thompson, and Breger, p. 148, pl. 59, fig. 2. 
1983 Globorotalia (Globorotalia) tumida tumida (Brady) - Kennett and Srinivasan, p. 158, pl. 36, figs. 1, 2, pl. 38, figs. 1-3.

1985 Globorotalia tumida (Brady) - Ujié, pl. 16, fig. 5.

1985 Globorotalia tumida tumida (Brady) - Bolli and Saunders, p. 227, figs. 338, 34.1134.13.

1985 Globorotalia (Globorotalia) tumida tumida (Brady) - lbaraki, p. 134, pl. 11, figs. 6, 7.

1994 Globorotalia (Globorotalia) tumida (Brady) - Jones, p. 103, pl. 103, figs. 4-6.

1994 Globorotalia tumida (Brady) - Loeblich and Tappan, p. 101, p. 183, figs. 7-12.

2004 Globorotalia tumida (Brady) - Hanagata, pl. 2, fig. 2.

Occurrence. Common in the Onogoshi, Yonahama, and Minebari formations.

Globorotalia tumida flexuosa (Koch)

Figures 16.9, 16.10

1923 Pulvinulina tumida var. flexuosa Koch, p. 357, text-figs. 9, 10.

1976 Globorotalia (Globorotalia) tumida flexuosa (Koch) - Natori, p. 230, pl. 4, fig. 7.

1977 Globorotalia tumida flexuosa (Koch) Kadar, p. 69, pl. 6, fig. 36.

1983 Globorotalia (Globorotalia) tumida flexuosa (Koch) - Kennett and Srinivasan, p. 158, pl. 38, figs. 4-6.

1985 Globorotalia (Globorotalia) tumida flexuosa (Koch) - Ibaraki, p. 133, pl. 11, figs. 2, 3.

1985 Globorotalia (s.s.) tumida flexuosa (Koch) — Ujiié, p. 113, pl. 16, fig. 6.

1985 Globorotalia tumida flexuosa (Koch) Bolli and Saunders, p. 227, figs. 33.6, 34.14-34.16.

Occurrence. Rare in the Yonahama Formation.

Globorotalia viola Blow

Figures 16.11, 16.12

1964 Globorotalia punctulata (d'Orbigny) LeRoy, p. F43, pl. 9, figs. 11-13 (non Globigerina punctulata d'Orbigny, 1826).

1969 Globorotalia (Globorotalia) crassula viola Blow, p. 397, pl. 5, figs. 4-6.

1974 Globorotalia (Globorotalia) crassula viola Blow - Ujiié and Ōki, pl. 3, fig. 4.

1978 Globorotalia (Globorotalia) crassula viola Blow - Keller (a), pl. 4, figs. 3, 4.
1981 Globorotalia viola Blow - Saito, Thompson, and Breger, pp. 134, 136, pl. 45, figs. 3, 4 .

1985 Globorotalia (Globorotalia) crassula viola Blow - Ibaraki, p. 129, pl. 9, figs. 5, 6.

1985 Globorotalia (Globorotalia) viola Blow Ujiié, pl. 12, figs. 5, 7.

1985 Globorotalia crassaformis viola Blow Bolli and Saunders, p. 234, fig. 36.1.

2004 Truncorotalia viola (Blow) - Hanagata, pl. 3, fig. 5.

Remarks. Globigerina punctulata of d'Orbigny (1826, nomen nudum), identified by LeRoy (1964), is now assigned to Globorotalia puncticulata (Deshayes) (see Cifelli, 1992); it differs from G. viola.

Occurrence. Common in the upper part of the Yonahama Formation up to the Minebari Formation.

Genus NEOGLOBOQUADRINA Bandy, Frerichs, and Vincent, 1967

Neogloboquadrina acostaensis (Blow)

Figures 17.1, 17.2

1959 Globorotalia acostaensis Blow, pp. 208210, pl. 17, figs. 106, 107.

1967 Globorotalia acostaensis Blow - Parker, pp. 164,165 , pl. 24, figs. 3-9.

1968 Globoquadrina acostaensis Blow Huang, p. 61, pl. 10, fig. 2.

1975 Globorotalia acostaensis Blow - Kameyama, pl., fig. 3.

1976 Globorotalia (Turborotalia) acostaensis Blow - Natori, p. 229, pl. 1, fig. 8.

1978 Globorotalia acostaensis Blow - Oda, p. 56 , pl. 5, figs. 2,3 .

1983 Neogloboquadrina acostaensis (Blow) Kennett and Srinivasan, p. 196, pl. 47, fig. 1, pl. 48, figs. $1-3$.

1985 Globorotalia (Turborotalia) acostaensis Blow - Ujiié, p. 111, pl. 10, figs. 8-11.

1985 Neogloboquadrina acostaensis Blow Ibaraki, p. 135, pl. 12, figs. 1-3.

1985 Globorotalia acostaensis acostaensis Blow - Bolli and Saunders, p. 210, figs. 27.10, 27.11, 28.16-28.24.

2004 Neogloboquadrina acostaensis (Blow) Hanagata, pl. 2, fig. 4.

2004 Turborotalita cf. pseudopumillio (Brönnimann and Resig) - Hanagata, pl. 3, fig. 8. 
Occurrence. Common to abundant in all the formations of the present study.

Neogloboquadrina blowi (Rögl and Bolli)

Figures 17.3, 17.4

1969 Globorotalia (Turborotalia) subcretacea (Lomnicki) - Blow, p. 392, pl. 4, figs. 1020 (non Globigerina subcretacea Lomnicki, 1901).

1973 Neogloboquadrina dutertrei (d'Orbigny) blowi Rögl and Bolli, p. 570, pl. 9, figs. 15-21, pl. 17, fig. 12.

1981 Neogloboquadrina blowi Rögl and Bolli Saito, Thompson, and Breger, p. 114, pl. 37, fig. 1.

1985 Neogloboquadrina blowi Rögl and Bolli Ibaraki, p. 136, pl. 12, figs. 6, 7.

1985 Neogloboquadrina dutertrei blowi Rögl and Bolli - Bolli and Saunders, p. 212, figs. 27.5, 28.9.

1994 Neogloboquadrina blowi Rögl and Bolli Jones, p. 91, pl. 82, fig. 10; Loeblich and Tappan, p. 102, figs. 7-10.

Remarks. This species is distinguished from its descendant, Neogloboquadrina dutertrei, in having a lower trochospiral and extra-umbilical aperture similar to that of Neogloboquadrina acostaensis.

Occurrence. Sporadic in the Oura, Onogoshi, and Yonahama formations.

Neogloboquadrina humerosa (Takayanagi and Saito)

Figures 17.5, 17.6

1962 Globorotalia humerosa Takayanagi and Saito, p. 78, pl. 28, fig. 1 (only).

1962 Globorotalia dutertrei (d'Orbigny) Parker, pp. 242, 244, pl. 7, fig. 11 (non Globigerina dutertrei d'Orbigny, 1839a).

1962 Globigerina subcretacea Lomnicki - Belford, p. 10, pl. 2, figs. $1-5$ (non G. subcretacea Lomnicki, 1901).

1964 Globigerina dubia Egger - LeRoy, p. F42, pl. 14, figs. 6 -8 (non Egger, 1857).

1968 Globoquadrina humerosa (Takayanagi and Saito) - Huang, p. 61, pl. 10, fig. 12.

1967 Globoquadrina humerosa (Takayanagi and Saito) - Parker, pp. 169, 170, pl. 24, figs. 10, 11, 12, pl. 25, fig. 6 .

1969 Globorotalia (Turborotalia) acostaensis humerosa Takayanagi and Saito - Blow, pp. 345,346 , pl. 25 , figs. $1-6$.
1976 Globorotalia (Turborotalia) humerosa humerosa Takayanagi and Saito - Natori, p. 229, pl. 2, fig. 4 .

1981 Neogloboquadrina humerosa (Takayanagi and Saito) - Saito, Thompson, and Breger, pp. 114, 116, pl. 37, fig. 2.

1983 Neogloboquadrina humerosa (Takayanagi and Saito) - Kennett and Srinivasan, p. 196, pl. 48, figs. 4-6.

1985 Globorotalia (Turborotalia) acostaensis humerosa Takayanagi and Saito - Ujiié, pp. 111, 112, pl. 11, fig. 3 (only).

1985 Globorotalia humerosa humerosa Takayanagi and Saito - Bolli and Saunders, p. 211, figs. 27.8, 28.15.

1985 Neogloboquadrina humerosa (Takayanagi and Saito) - Ibaraki, p. 137, pl. 12, figs. 15-17.

1994 Neogloboquadrina humerosa (Takayanagi and Saito) - Loeblich and Tappan, p. 102, pl. 199, figs. $1-6$.

2004 Neogloboquadrina humerosa humerosa (Takayanagi and Saito) - Hanagata, pl. 2, fig. 5 .

Occurrence. Sporadic in the Minebari and Yonahama formations.

Neogloboquadrina incompta (Cifelli)

Figures 17.7-17.14

1961 Globigerina incompta Cifelli, p. 84, pl. 4, figs. $1-5$ (non 6,7$)$.

1973 Globigerina pachyderma (Ehrenberg) Cifelli, pl. 1, figs. 1-7 (non Aristerospira pachyderma Ehrenberg, 1861).

1973 Globigerina incompta Cifelli - Cifelli, pl. 2, figs. 5-7.

1977 Globigerina pachyderma (Ehrenberg) Kadar, p. 63, pl. 2, fig. 13.

1978 Neogloboquadrina pachyderma (Ehrenberg) - Keller (b), pl. 1, figs. 7-9; Keller (c), pl. 1, fig. 3, pl. 4, figs. $1-9$.

1981 Neogloboquadrina incompta (Cifelli) Saito, Thompson, and Breger, p. 108, pl. 34, fig. 2.

1982 Globigerina incompta Cifelli - Cifelli, pl. 3, figs. 1, 2 (non 3).

2005 Neogloboquadrina incompta (Cifelli) Domitsu and Oda, fig. 3.8.

2006 Neogloboquadrina incompta (Cifelli) Domitsu and Oda, figs. 6-5, 6-6. 


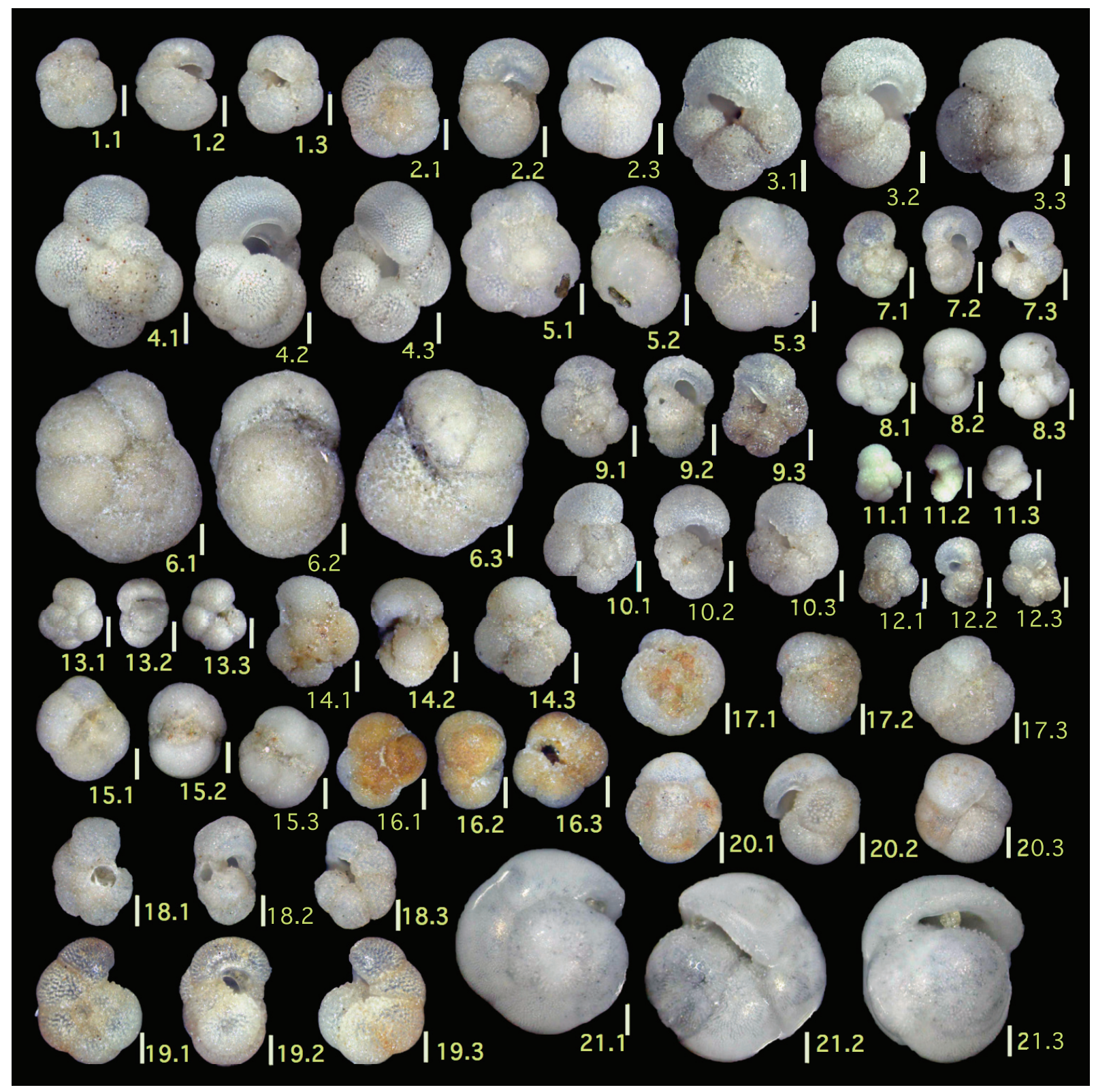

FIGURE 17. Family Globorotaliidae and Pulleniatinidae. All scale bars $=0.1 \mathrm{~mm} .1$, 2. Neogloboquadrina acostaensis (Blow), 1: MPC-25996 from sample MK01 (Yonahama Formation, PL2); 2: MPC-25997 from sample MK02B (Minebari F., PL5). 3, 4. Neogloboquadrina blowi (Rögl and Bolli), 3: MPC-25998 from sample 6 (Yonahama F., PL2); 4: MPC25999 from sample 6-2 (Yonahama F., Yonahama F., PL2). 5, 6. Neogloboquadrina humerosa (Takayanagi and Saito), 5: MPC-26000 from sample 15 (Yonahama F., PL3); 6: MPC-26001 from sample MK02A (Minebari F., PL5). 7-14. Neogloboquadrina incompta (Cifelli), 7: MPC-26002 from sample 14-2 (Yonahama F., PL4); 8: MPC-26003 from sample 16 (Yonahama F., PL3); 9: MPC-26004 from sample 3-2-low-silt (Onogoshi F., PL1); 10: MPC-26005 from sample 10 (Onogoshi F., PL2); 11: MPC-26006 from sample 15 (Yonahama F., PL3); 12: MPC-26007 from sample MK01 (Yonahama F., PL2); 13: MPC-26008 from sample 6-2 (Yonahama F., PL2); 14: MPC-26009 from sample 32-up-sand (Onogoshi F., PL1). Specimens of 8-10, right coiling (dextral) form; 11-14, left coiling (sinistral) form. 15-17. Neogloboquadrina pseudopachyderma (Cita, Premoli-Silva, and Rossi), 15: MPC-26010 from sample 12 (Minebari F., PL5); 16: MPC-26011 from sample MK01 (Yonahama F., PL2); 17: MPC-26012 from sample 2-2 (Minebari F., PL5). 15 and 16, right coiling (dextral) form; 17, left coiling (sinistral) form. 18, 19. Neogloboquadrina praehumerosa (Natori), 18: MPC-26013 from sample MK01 (Yonahama F., PL2); 19: MPC-26014 from sample MK02B (Minebari F., PL5). 20, 21. Pulleniatina obliquiloculata (Parker and Jones), 20: MPC-26018 from sample 17 (Yonahama F., PL2); 21: MPC-26019 from sample 5 (Minebari F., PL5). 20, left coiling (sinistral) form; 21, right coiling (dextral) form. 
PE Note: Corrigendum to Hanagata and Nobuharai. 2015

3 January 2016

Corrigendum to "Illustrated guide to Pliocene foraminifera from Miyakojima, Ryukyu Island Arc, with comments on biostratigraphy,"

Hanagata, Satoshi and Nobuhara, Takami. 2015. Palaeontologia Electronica 18.1.3A: 1-140.

palaeo-electronica.org/content/2015/1016-foraminifera-of-miyakojima

Specimen registration numbers were incorrectly cited in the following three figure captions:

\begin{tabular}{|c|c|c|}
\hline Figure & Cited number & Correct number \\
\hline Figure 17.20 & MPC-26018 & MPC-26020 \\
\hline Figure 18.10 & MPC-25926 & MPC-25929 \\
\hline Figure 19.12 & MPC-25941 & MPC-25946 \\
\hline
\end{tabular}


2008 Neogloboquadrina incompta (Cifelli) Domitsu and Oda, p. 6, pl. 4, figs. 4-6.

Remarks. According to Cifelli's (1961) type figures, he included the dextral coiling form of Neogloboquadrina pseudopachyderma in the paratypes of $N$. incompta. It is obvious from his later work (Cifelli, 1973, 1982) that he differentiated $N$. incompta from Neogloboquadrina pachyderma and other 'neogloboquadrids' not by test shape, but by coiling direction and surface encrustation. Domitsu and Oda (2008) and Oda and Domitsu (2009), on the other hand, treated the lobulate and umbilicusextra umbilicus aperture form as $N$. incompta irrespective of coiling direction. We basically agree with the latter view and do not attach importance to the coiling direction; however, formal emendation of the species based on type specimens has never been performed - this needs to be done in a future study.

Occurrence. Rare in the Onogoshi Formation, sporadic in the Minebari and Yonahama formations.

Neogloboquadrina pseudopachyderma (Cita, Premoli-Silva, and Rossi)

Figures 17.15-17.17

1965 Globorotalia pseudopachyderma Cita, Premoli-Silva, and Rossi, p. 233, pl. 20, figs. $3,4,6$, pl. 31, fig. 6, p. 235, text-fig. 5 .

1974 Globigerina pseudopachyderma Cita, Premoli-Silva, and Rossi - Olsson, pl. 2, figs. A-J.

1975 Neogloboquadrina atlantica (Berggren) Poore and Berggren, pl. 1, fig. 8-13, pl. 2, fig. 7.

1985 Neogloboquadrina pachyderma (Ehrenberg) - Ibaraki, p. 138, pl. 13, figs. 3, 4.

2000 Neogloboquadrina inglei Kucera and Kennett, p. 80, pl. 1, figs. 1-13.

2003 Neogloboquadrina incompta (Cifelli) Ujiié, p. 41, pl. 1, fig. 2 (non Globigerina incompta Cifelli, 1961).

2004 Neogloboquadrina incompta (Cifelli) Hanagata, pl. 2, figs. 1, 2.

Remarks. Almost all specimens from the Pliocene of Miyakojima Island exhibit dextral coiling except for a single specimen from sample 2-2 of the Minebari Formation which is sinistral.

There have been extensive taxonomic studies of the morphological variations of $N$. pachyderma and related species, including N. borealis [= Globigerina bulloides d'Orbigny var. borealis Brady, 1881, first referred to as Globigerina bulloides arc- tic variety by Brady, 1878 , p. 435 , pl. 21, fig. 10; also reprinted as $N$. pachyderma by Jones, 1994, p. 113 , pl. 114, figs. 19, 20], N. pseudopachyderma, N. incompta, N. atlantica [= Globigerina atlantica Berggren, 1972, pp. 972, 973, pl. 1, fig. 1-7; Poore and Berggren, 1975], and N. inglei. In addition, attempts have been made to distinguish morphotypes based on wall calcification/encrustation, chamber inflation, lobulation, or test size (e.g., Kennett, 1968; Bandy, 1972; Srinivasan and Kennett, 1974; Reynolds and Thunell, 1986; Bergami et al., 2009; Eynaud et al., 2009). However, the relationship among these species is still confused. For example, it is speculated that Poore and Berggren (1975) included N. pseudopachyderma and $N$. incompta, and also Neogloboquadrina praehumerosa (as here understood) in their N. atlantica, as judged from their illustrations.

Among those studies, Keller (1978b) regarded incompta and puseudopachyderma as morphotypes of $N$. pachyderma, focusing on test size assuming the differences in test shape mirrored growing stages. Her work should be valued for treating incompta and pseudopachyderma in the discussion; later work did not even mention those species.

Recent molecular study, based on r-DNA, is expected to resolve this problem (e.g., Darling et al., 2006, 2007). So far, DNA extraction and morphological classification have not been properly combined in previous studies.

Occurrence. Dextral specimens are common in the Onogoshi and Minebari formations. A single sinistral specimen is available from the Minebari Formation.

\section{Neogloboquadrina praehumerosa (Natori)} Figures 17.18, 17.19

1962 Globorotalia humerosa Takayanagi and Saito, p. 78, pl. 28, fig. 2 (only).

1976 Globorotalia (Turborotalia) humerosa praehumerosa Natori, p. 227, pl. 2, figs. 1, 3.

1977 Globoquadrina dutertrei (d'Orbigny) Kadar, p. 65, pl. 5, fig. 27 (non Globigerina dutertrei d'Orbigny, 1839a).

1978 Globorotalia humerosa Takayanagi and Saito - Oda, pp. 57, 58, pl. 5, figs. 4, 5.

1985 Globorotalia acostaensis humerosa Takayanagi and Saito - Ujiié, pp. 111, 112, pl. 11, fig. 2 (non fig. 3).

1985 Neogloboquadrina humerosa praehumerosa (Natori) - Bolli and Saunders, p. 211, figs. $27.9,28.14$. 
2004 Neogloboquadrina praehumerosa (Natori) - Hanagata, pl. 2, fig. 6.

Remarks. This species is distinguished from Neogloboquadrina humerosa in having fewer chambers, five to five and a half, in the last whorl, rather than six.

Occurrence. Common in the Yonahama and Minebari formations.

Family PULLENIATINIDAE Cushman, 1927a

Genus PULLENIATINA Cushman, 1927a

Pulleniatina obliquiloculata (Parker and Jones)

Figures 17.20, 17.21

1865 Pullenia sphaeroides (d'Orbigny) var. obliquiloculata Parker and Jones, pp. 365, 368, pl. 19, fig. 4.

1941 Pulleniatina obliquiloculata (Parker and Jones) - LeRoy (Part 1), pp. 44, 45, pl. 2, figs. 105-107; LeRoy (Part 2), p. 87, pl. 4, figs. 16-18; LeRoy (Part 3), p. 118, pl. 2, figs. 13-15.

1959 Pulleniatina obliquiloculata (Parker and Jones) - Bradshow, p. 49, pl. 8, figs. 19, 20; Graham and Militante, p. 113, pl. 19, fig. 4.

1962 Pulleniatina obliquiloculata (Parker and Jones) - Parker, p. 234, pl. 4, figs. 1316, 19, 22; Belford, pp. 23, 24, pl. 6, figs 12-17.

1963 Pulleniatina obliquiloculata (Parker and Jones) - Saito, pp. 199, 200, pl. 54, figs. 6 -8.

1964 Pulleniatina obliquiloculata (Parker and Jones) - LeRoy, pp. F42, F43, pl. 14, figs. 25-28.

1965 Pulleniatina obliquiloculata (Parker and Jones) - Todd, p. 67, pl. 27, figs. 2-4.

1967 Pulleniatina obliquiloculata (Parker and Jones) - Parker, pp. 172, 173, pl. 28, fig. 1 , Huang, p. 188 , pl. 15 , fig. 11 ; pl. 16 , figs. 8-11.

1975 Pulleniatina obliquiloculata obliquiloculata (Parker and Jones) - Ibaraki and Tsuchi, pl. 4, fig. 16.

1976 Pulleniatina obliquiloculata obliquiloculata (Parker and Jones) - Natori, p. 232, pl. 6, fig. 2.

1977 Pulleniatina obliquiloculata (Parker and Jones) - McCulloch, p. 435, pl. 174, fig. 4.

1977 Pulleniatina obliquiloculata obliquiloculata (Parker and Jones) - Kadar, p. 64, pl. 4, fig. 23.
1978 Pulleniatina obliquiloculata (Parker and Jones) - Oda, pp. 63, 64, pl. 10, figs. 6, 10.

1983 Pulleniatina obliquiloculata (Parker and Jones) - Kennett and Srinivasan, p. 202, pl. 49, fig. 2, pl. 50, figs. 6 -9.

1985 Pulleniatina obliquiloculata (Parker and Jones) - Ujiié, pl. 18, fig. 7; Ibaraki, p. 138, pl. 13, figs. 5, 6; Bolli and Saunders, pp. 247, 248, figs. 40.4, 41.9-41.12.

1988 Pulleniatina obliquiloculata (Parker and Jones) - Wang, Zhang, Zhao, Min, Bian, Zheng, Cheng, and Chen, pp. 50, 51, pl. 2, figs. 5-13, pl. 9, fig. 8.

1994 Pulleniatina obliquiloculata (Parker and Jones) - Jones, p. 92, pl. 84, figs. 1620; Loeblich and Tappan, p. 103, pl. 187, figs. 8-13, pl. 188, figs. 1-6.

2004 Pulleniatina obliquiloculata (Parker and Jones) - Hanagata, pl. 4, fig. 1.

Occurrence. Common in the Onogoshi, Yonahama and Minebari formations.

\section{Pulleniatina okinawaensis Natori}

Figures 18.1, 18.2

1976 Pulleniatina okinawaensis Natori, pp. 227, 228, pl. 5, figs. 5, 6 .

1985 Pulleniatina okinawaensis Natori - Ujié, p. 114, pl. 18, figs. 3,4 .

1994 Pulleniatina okinawaensis Natori - Loeblich and Tappan, p. 103, pl. 198, figs. 16.

2004 Pulleniatina okinawaensis Natori - Hanagata, pl. 4, fig. 2.

Remarks. Only dextral forms have been recovered. This probably is a neoteny of Pulleniatina obliquiloculata, retaining the immature character of the apertural position, centred in the umbilical region retained in the adult stage.

Occurrence. Rare in the Yonahama Formation.

Pulleniatina praecursor Banner and Blow

Figures 18.3, 18.4

1967 Pulleniatina obliquiloculata (Parker and Jones) praecursor Banner and Blow, $\mathrm{p}$. 139, pl. 3, fig. 3.

1974 Pulleniatina obliquiloculata praecursor Banner and Blow - Ujiié and Ōki, pl. 6, fig. 5.

1976 Pulleniatina obliquiloculata praecursor Banner and Blow - Natori, pl. 5, fig. 4. 
1977 Pulleniatina obliquiloculata praecursor (Banner and Blow) - Kadar, p. 64, pl. 4, fig. 22.

1983 Pulleniatina praecursor Banner and Blow - Kennett and Srinivasan, p. 200, pl. 49, figs. 6-8.

1981 Pulleniatina praecursor Banner and Blow - Saito, Thompson, and Breger, p. 101, pl. 32, figs. 1, 2.

1985 Pulleniatina praecursor Banner and Blow - Ujiié, pl. 18, fig. 6; Bolli and Saunders, p. 247 , figs. $40.5,41.1,41.2$.

Occurrence. Three specimens from a single sample of the Yonahama Formation.

Family CANDEINIDAE Cushman, 1927a

Subfamily GLOBIGERINITINAE Bermúdez, 1961

Genus GLOBIGERINITA Brönnmann, 1951a Globigerinita glutinata (Egger)

Figures 18.5-18.7

1893 Globigerina glutinata Egger, p. 371, pl. 13, figs. $19-21$.

1962 Globigerinita glutinata (Egger) - Parker, pp. 246-249, pl. 9, figs. 1-16; Belford, pp. 11, 12, pl. 2, figs. 11-16.

1963 Globigerina glutinata (Egger) - Saito, p. 185 , p. 56, fig. 4.

1967 Globigerinita glutinata (Egger) - Parker, p. 146, pl. 17, figs. 3-5; Huang, p. 187, pl. 16, fig. 6.

1968 Globigerinita glutinata (Egger) - Huang, p. 60 , pl. 11 , figs. $6,7$.

1975 Globigerinita glutinata (Egger) - Ibaraki and Tsuchi, pl. 3, fig. 7.

1977 Globigerina glutinata (Egger) - Kadar, pp. 61, 63, pl. 2, fig. 12.

1978 Globigerinita glutinata (Egger) - Oda, pp. 52, 53, pl. 2, fig. 10.

1981 Globigerinita glutinata (Egger) - Saito, Thompson, and Breger, p. 77, pl. 22, figs. $1-7$.

1983 Globigerinita glutinata (Egger) - Kennett and Srinivasan, p. 224, pl. 56, figs. 1, 3-5.

1985 Globigerinita glutinata (Egger) - Ujiié, pl. 7, figs. 1, 2; Ibaraki, p. 122, pl. 6, figs. 911.

1988 Globigerinita glutinata (Egger) - Wang, Zhang, Zhao, Min, Bian, Zheng, Cheng, and Chen, p. 45, pl. 1, figs. 1-7.
1994 Tinophodella ambitacretacea Loeblich and Tappan - Loeblich and Tappan, p. 104, pl. 192, figs. $1-9$, pl. 200 , figs. $1-6$.

2004 Globigerinita glutinata (Egger) - Hanagata, pl. 4, fig. 3.

Remarks. We follow Kennett and Srinivasan (1983) in regarding Tinophodella ambitacretacea (Loeblich and Tappan, 1957) a junior synonym of G. glutinata. Fossil specimens of this species occasionally lack bullae on the umbilicus due to secondary abrasion.

Occurrence. Common to abundant in all the formations of the present study.

\section{Globigerinita uvula (Ehrenberg)}

Figures 18.8, 18.9

1861 Pylodexia uvula Ehrenberg, p. 308.

1873 Pylodexia uvula Ehrenberg - Ehrenberg, pl. 2, figs. 24, 25.

1931 Globigerina bradyi Wiesner, p. 133.

1962 Globigerinita uvula (Ehrenberg) - Parker, p. 252 , pl. 8 , figs. $14-26$.

1967 Globigerinita uvula (Ehrenberg) - Parker, p. 146, pl. 17, figs. $8,9$.

1977 Globigerina (?) bradyi Wiesner - McCulloch, p. 411, pl. 173, fig. 12.

1983 Globigerinita uvula (Ehrenberg) - Kennett and Srinivasan, p. 224, pl. 56, figs. 6-8.

1985 Globigerinita uvula (Ehrenberg) - Ujiié, pl. 7, fig. 3; Ibaraki, p. 123, pl. 6, figs. 14, 15; Rögl, p. 323, figs. 5.25, 5.26.

1994 Globigerina bradyi Wiesner - Jones, p. 91, pl. 82, figs. 8, 9.

1994 Globigerinita uvula (Ehrenberg) - Loeblich and Tappan, p. 104, pl. 191, figs. 712.

Remarks. Globigerina bradyi is here regarded a junior synonym of this species, although this relationship issue is controversial, mainly on the basis of problems surrounding the figure of the type of $G$. uvula (see Jenkins et al., 1986; Jones, 1994).

Occurrence. Rare in the Onogoshi, Yonahama, and Minebari formations.

Subfamily CANDENINAE Cushman, 1927a Genus CANDEINA d'Orbigny, 1839a Candeina nitida d'Orbigny

Figure 18.10

1839 Candeina nitida d'Orbigny (a), p. 108, pl. 2, figs. 27, 28.

1903 Candeina nitida d'Orbigny - Millett (part XV), pp. 692, 693, pl. 7, fig. 2. 


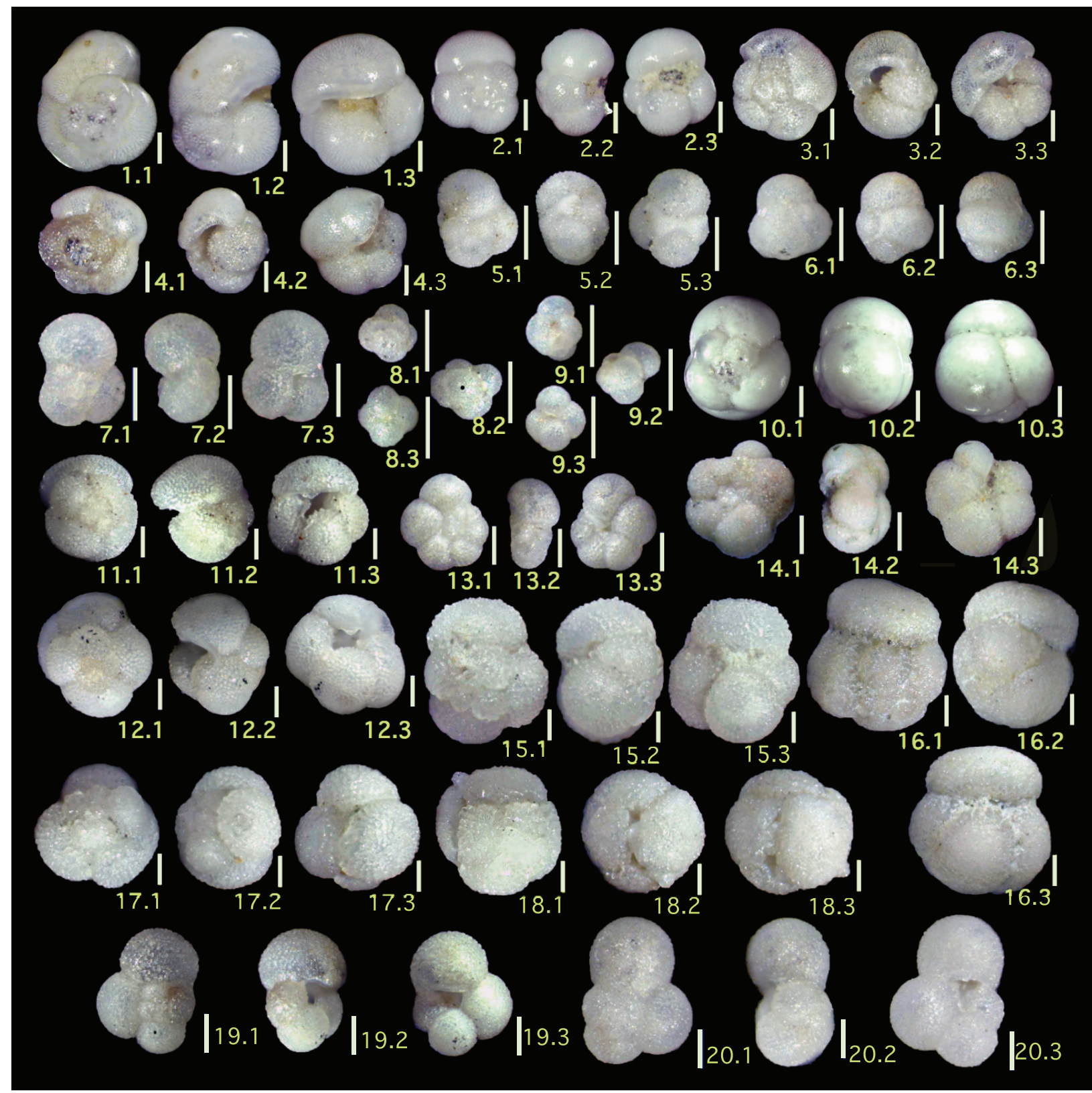

FIGURE 18. Family Pulleniatinidae, Candeinidae, Catapsydracidae, and Globigerinidae. All scale bars $=0.1 \mathrm{~mm} .1$, 2. Pulleniatina okinawaensis Natori, 1: MPC-26021 from sample 4-2 (Minebari Formation, PL5); 2: MPC-26022 from sample 12 (Minebari F., PL5); both right coiling (dextral) specimens. 3, 4. Pulleniatina praecursor Banner and Blow, 3: MPC-26023, 4: MPC-26024, both from sample 6 (Yonahama F., PL2). 5-7. Globigerinita glutinata (Egger), 5: MPC25950 from sample MK01 (Yonahama F., PL2); 6: MPC-25951 and 7: MPC-25952 from sample MK02B (Minebari F., PL5). Specimen of 7 lacks bulla-like cover on the umbilicus. 8, 9. Globigerinita uvula (Ehrenberg), 8: MPC-25953 from sample 10 (Onogoshi F., PL2); 9: MPC-25954 from sample MK01 (Yonahama F., PL2). 10. Candeina nitida d'Orbigny, MPC-25926 from sample 5-2 (Minebari F., PL5). 11, 12. Dentoglobigerina altispira (Cushman and Jarvis), 11: MPC-25932 from sample 10 (Onogoshi F., PL2); 12: MPC-25933 from sample MK01 (Yonahama F., PL2). $13,14$. Globorotaloides hexagona (Natland), 13: MPC-25988 from sample 1 (Minebari F., PL5); 14: MPC-25989 from sample 5 (Minebari F., PL5). 15-18. Alloglobigerinoides conglobatus (Brady), 15: MPC-25925 from sample 12 (Minebari F., PL5); 16: MPC-25926 from sample MK02A (Minebari F., PL5); 17: MPC-25927 from sample 5 (Minebari F., PL5); 18: MPC-25928 from sample 14 (Yonahama F., PL4). 17 and 18, variation final chamber covering umbilicus. 19, 20. Globigerina bulloides d'Orbigny, 19: MPC-25936 from sample MK01 (Yonahama F., PL2); 20: MPC-25937 from sample MK02B (Minebari F., PL5). 
1959 Candeina nitida d'Orbigny - Bradshow, pp. 32, 33, pl. 7, fig. 19.

1962 Candeina nitida d'Orbigny - Parker, p. 253, pl. 8, figs. 27-30; Belford, p. 29, 30, pl. 8, figs. 17, 18.

1964 Candeina nitida d'Orbigny - LeRoy, p. F43, pl. 6, fig. 11.

1965 Candeina nitida d'Orbigny - Todd, p. 68, pl. 27, fig. 1.

1967 Candeina nitida d'Orbigny - Parker, p. 145, pl. 17, figs. 1, 2.

1968 Candeina nitida d'Orbigny - Huang, p. 60, pl. 10, fig. 8.

1969 Candeina nitida d'Orbigny - Blow, pp. 335 , 384-386, pl. 23, figs. $1-4$.

1975 Candeina nitida d'Orbigny - Ibaraki and Tsuchi, pl. 3, fig. 8.

1977 Candeina cf. nitida d'Orbigny - McCulloch, p. 405, pl. 169, figs. 2-4.

1981 Candeina nitida d'Orbigny - Saito, Thompson, and Breger, p. 75, pl. 21, figs. $1,2$.

1983 Candeina nitida d'Orbigny - Kennett and Srinivasan, p. 229, pl. 57, figs. 6-8.

1985 Candeina nitida d'Orbigny - Bolli and Saunders, p. 191, figs. 19.1, 19.2.

1985 Candeina nitida nitida d'Orbigny - Ujiié, pl. 7, fig. 6 .

1988 Candeina nitida d'Orbigny - Wang, Zhang, Zhao, Min, Bian, Zheng, Cheng, and Chen, p. 44, pl. 4, figs. 15, 16.

1994 Candeina nitida d'Orbigny - Jones, p. 91, pl. 82, figs. 13-20; Loeblich and Tappan, p. 104 , pl. 193 , figs. $1-4$.

2004 Non Candeina nitida d'Orbigny - Hanagata, pl.4, fig. 4.

Remarks. A single, well-preserved specimen is available from sample 5-2nd. The first author (Hanagata, 2004) erroneously identified this as a variation of Alloglobigerinoides conglobatus as understood in the present study.

Occurrence. Minebari Formation.

Family CATAPSYDRACIDAE Bolli, Loeblich, and Tappan, 1957

Genus DENTOGLOBIGERINA Blow, 1979

Dentoglobigerina altispira (Cushman and Jarvis)

Figures 18.11, 18.12

1936 Globigerina altispira Cushman and Jarvis, p. 5, pl. 1, figs. 13, 14.
1959 Globoquadrina altispira altispira (Cushman and Jarvis) - Blow, p. 183, pl. 8, fig. 51.

1962 Globoquadrina altispira altispira (Cushman and Jarvis) - Belford, pp. 21, 22, pl. 5, figs. $19-24$.

1963 Globoquadrina altispira altispira (Cushman and Jarvis) - Saito, p. 189, pl. 55, fig. 8.

1964 Globoquadrina altispira (Cushman and Jarvis) - LeRoy, p. F42, pl. 14, figs. 12, 13.

1964 Globigerina baroemoenensis LeRoy LeRoy, p. F42, pl. 14, figs. 9, 10 (non Globigerina baroemoenensis LeRoy, 1939).

1967 Globoquadrina altispira (Cushman and Jarvis) - Parker, p. 165, pl. 25, fig. 8; Huang, p. 188, pl. 16, fig. 19.

1968 Globoquadrina altispira (Cushman and Jarvis) - Huang, p. 61, pl. 10, figs. 13, 16.

1977 Globoquadrina altispira altispira (Cushman and Jarvis) - Kadar, p. 65, pl. 3, fig. 16.

1978 Globoquadrina altispira (Cushman and Jarvis) - Oda, p. 55, pl. 4, figs. 1-3.

1983 Dentoglobigerina altispira altispira (Cushman and Jarvis) - Kennett and Srinivasan, p. 188 , pl. 46 , figs. 4-6.

1985 Globoquadrina altispira altispira (Cushman and Jarvis) - Ibaraki, p. 116, pl. 4, figs. 13, 14; Bolli and Saunders, p. 183, fig. 15.1.

2004 Globoquadrina altispira (Cushman and Jarvis) - Hanagata, pl. 4, fig. 5.

Occurrence. Common in the Onogoshi and Yonahama formations.

Genus GLOBOROTALOIDES Bolli, 1957 Globorotaloides hexagona (Natland)

Figures 18.13, 18.14

1938 Globigerina hexagona Natland, p. 149, pl. 7, fig. 1.

1959 Globigerina hexagona Natland - Bradshow, p. 36, figs. $1-15$.

1962 Globoquadrina hexagona (Natland) Parker, p. 244, pl. 8, figs. 5-12 (?13).

1967 Globoquadrina hexagona (Natland) Parker, p. 169, pl. 25, figs. 9, 10.

1968 Globoquadrina hexagona (Natland) Huang, p. 61, pl. 11, fig. 12.

1971 Globorotalia (Turborotalia) planispira Brönnimann and Resig, p. 1282, pl. 36, figs. 4, 6 , pl. 44 , figs. $1,2,4,5,7,8$, pl. 47, fig. 1 . 
1974 Globorotalia (Turborotalita) planispira Brönnimann and Resig - Ujiié and Ōki, pl. 3, fig. 1.

1981 Globorotaloides hexagona (Natland) Saito, Thompson, and Breger, p. 91, pl. 28, figs. 1, 2.

1983 Globoquadrina hexagona (Natland) Kennett and Srinivasan, p. 216, pl. 54, figs. 1, 3-5.

1985 Globorotaloides variabilis Bolli, var. B Ujiié, p. 111, pl. 9, figs. 11-13.

1985 Globoquadrina hexagona (Natland) Ujiié, pl. 10, figs. 1, 2; Ibaraki, p. 119, pl. 5, figs. $8,9$.

1988 Neogloboquadrina hexagona (Natland) - Wang, Zhang, Zhao, Min, Bian, Zheng, Cheng, and Chen, p. 50 , pl. 7 , figs. $14-$ 15.

2003 Globorotaloides hexagona (Natland) Ujiié, p. 43, pl. 1, fig. 6 .

Occurrence. Common in the Yonahama and Minebari formations.

Superfamily GLOBIGERINOIDEA Carpenter, Parker, and Jones, 1862

Family GLOBIGERINIDAE Carpenter, Parker, and Jones, 1862

Subfamily GLOBIGERININAE Carpenter, Parker, and Jones, 1862

Genus ALLOGLOBIGERINOIDES Huang, 1986

Alloglobigerinoides conglobatus (Brady) Figures 18.15-15.18

1879 Globigerina conglobata Brady, pp. 286, 287.

1884 Globigerina conglobata Brady - Brady, pl. 80 , figs. $1-5$.

1959 Globigerinoides conglobata (Brady) Bradshow, p. 40, pl. 7, figs. 5, 6 .

1962 Globigerinoides conglobatus (Brady) Parker, p. 229, pl. 3, figs. 1-5; Belford, pp. 18,19 , pl. 4 , figs. $15-20$, text-figs. $3-1-3-$ 6.

1965 Globigerinoides conglobatus (Brady) Todd, p. 62, pl. 25, fig. 3.

1967 Globigerinoides conglobatus (Brady) Parker, p. 154, pl. 20, figs. 3, 4.

1968 Globigerinoides conglobatus (Brady) Huang, p. 60, pl. 11, figs. 10, 24.

1975 Globigerinoides conglobatus (Brady) Kameyama, pl., fig. 2.
1975 Globigerinoides conglobatus conglobatus (Brady) - Ibaraki and Tsuchi, pl. 2, fig. 1.

1977 Globigerinoides conglobata (Brady) McCulloch, p. 417, pl. 173, fig. 5.

1977 Globigerinoides conglobatus (Brady) Kadar, p. 67, pl. 1, figs. 6, 7.

1978 Globigerinoides conglobatus (Brady) Oda, p. 53, pl. 2, figs. 12, 13.

1981 Globigerinoides conglobatus (Brady) Saito, Thompson, and Breger, p. 56, pl. 14, fig. 1.

1983 Globigerinoides conglobatus (Brady) Kennett and Srinivasan, p. 58, pl. 12, figs. 4-6.

1985 Globigerinoides conglobatus (Brady) Ibaraki, p. 111, pl. 3, figs, 3, 4; Bolli and Saunders, p. 194, fig. 21.1.

1988 Globigerinoides conglobatus (Brady) Wang, Zhang, Zhao, Min, Bian, Zheng, Cheng, and Chen, pp. 42, 43, pl. 6, figs. 13-16.

1994 Globigerinoides conglobatus (Brady) Jones, p. 89 , pl. 80 , figs. $1-5$, p. 90 , pl. 82, fig. 5.

1994 Alloglobigerinoides conglobatus (Brady) Loeblich and Tappan, p. 105, pl. 193, figs. 5-10, pl. 194, figs. 1-3.

2004 Globigerinoides conglobatus (Brady) Hanagata, pl. 5, fig. 4.

2004 Candeina nitida d'Orbigny - Hanagata, pl. 4, fig. 4.

Remarks. The first author (Hanagata, 2004) erroneously identified this as the variation which has a bulla-like cover closing the umbilicus under the name of Candeina nitida. Belford (1962), Huang (1968, pl. 11, fig. 10), Kadar (1977), and Loeblich and Tappan (1994, pl. 193, figs. 8-10) illustrated variants with such bulla-like covers.

Occurrence. Common in the Minebari and Yonahama formations.

Genus GLOBIGERINA d'Orbigny, 1826 Globigerina bulbosa LeRoy

Figures 19.7, 19.8

1944 Globigerina bulbosa LeRoy (Part 2), p. 39, pl. 3, figs. 26, 27.

1959 Globigerina bulbosa LeRoy - Blow, pp. 174, 175, pl. 9, fig. 37.

1968 Globigerina bulbosa LeRoy - Huang, pl. 11, fig. 3. 
1969 Globigerina bulbosa LeRoy - Blow, pl. 13, figs. 3-6.

Remarks. As Blow (1959) noted, this species is closely related to Globigerina foliata but can be differentiated by its radially elongated form.

Occurrence. Sporadic in the Onogoshi, Yonahama, and Minebari formations.

\section{Globigerina bulloides d'Orbigny}

Figures 18.19, 18.20

1826 Globigerina bulloides d'Orbigny, p. 277.

1941 Globigerina bulloides d'Orbigny - LeRoy (Part 3), p. 118, pl. 3, figs. 27, 28.

1944 Globigerina bulloides d'Orbigny — LeRoy (Part 1), p. 39, pl. 7, figs. 40-42.

1959 Globigerina bulloides d'Orbigny - Bradshow, p. 33, pl. 6, figs. 1-4; Blow, p. 175, 176, pl. 9, fig. 38 .

1962 Globigerina bulloides d'Orbigny - Parker, p. 221, pl. 1, figs. 1-8; Belford, pp. 9, 10. pl. 1 , figs. $16-20$.

1964 Globigerina bulloides d'Orbigny - LeRoy, p. F42, pl. 14, fig. 11.

1967 Globigerina bulloides d'Orbigny - Huang, p. 187 , pl. 16, fig. 3.

1978 Globigerina bulloides d'Orbigny — Oda, p. 50 , pl. 1, figs. 2,3 .

1981 Globigerina bulloides d'Orbigny - Saito, Thompson, and Breger, p. 40, pl. 7, fig. 1.

1983 Globigerina bulloides d'Orbigny - Kennett and Srinivasan, p. 36, pl. 6, figs. 4-6.

1985 Globigerina bulloides d'Orbigny - Ujiié, p. 108, pl. 1, figs. 1, 2; Papp and Schmid, p. 62 , pl. 54 , figs. $1-6$; Ibaraki, p. 106, pl. 1 , figs. 5, 6; Rögl, p. 321, figs. 4.1, 4.2.

1988 Globigerina bulloides d'Orbigny - Wang, Zhang, Zhao, Min, Bian, Zheng, Cheng, and Chen, pp. 39, 40, pl. 7, figs. 1-5.

1994 Globigerina bulloides d'Orbigny - Jones, p. 88 , pl. 77 , pl. 79 , figs. 3-7.

2003 Globigerina bulloides d'Orbigny - Ujiié, p. 43 , pl. 1, fig. 8.

2004 Globigerina bulloides d'Orbigny - Hanagata, pl. 1, fig. 1.

Occurrence. Common in all the formations of the present study.

Globigerina falconensis Blow

Figures 19.1, 19.2

1959

Globigerina falconensis Blow, p. 177, pl. 9, figs. $40,41$.
1962 Globigerina falconensis Blow - Parker, p. 224, pl. 1, figs. $14,16-19$.

1963 Globigerina falconensis Blow - Saito, p. 185, pl. 56, fig. 5.

1967 Globigerina falconensis Blow - Huang, p. 187, pl. 16, fig. 4.

1978 Globigerina falconensis Blow - Oda, pp. 50, 51, pl. 1, figs. 8, 9.

1981 Globigerina falconensis Blow - Saito, Thompson, and Breger, p. 40, pl. 7, fig. 2.

1983 Globigerina falconensis Blow - Kennett and Srinivasan, pp. 40, 42, pl. 7, figs. 1-3.

1985 Globigerina falconensis Blow - Ibaraki, pp. 107,108 , pl. 1, figs. 15, 16; laccarino, p. 303 , fig. 5.2 .

2004 Globigerina falconensis Blow - Hanagata, pl. 5, fig. 2.

Occurrence. Common in the Onogoshi, Yonahama, and Minebari formations.

Globigerina foliata Bolli

Figures 19.3-19.6

1957 Globigerina foliata Bolli, p. 111, pl. 24, fig. 1.

1959 Globigerina foliata Bolli - Blow, pp. 177, 178, pl. 10, fig. 42.

1968 Globigerina foliata Bolli - Huang, p. 60, pl. 11, figs. $1,8$.

1975 Globigerina parabulloides Blow - Kameyama, pl. 1, fig. 1 (non G. parabulloides Blow, 1959).

1977 Globigerina foliata Bolli - Kadar, p. 63, pl. 2, fig. 10.

1978 Globigerina foliata Bolli — Oda, p. 51, pl. 1, fig. 7.

1985 Globigerina foliata Bolli - Ujiié, p. 108, pl. 1, figs. 5-10; Ibaraki, pl. 2, figs. 1, 2.

2003 Globigerina foliata Bolli - Ujiié, p. 43, pl. 1, fig. 9.

2004 Globigerina foliata Bolli — Hanagata, pl. 5, fig. 3.

Remarks. This species is commonly found in the Pliocene of the Ryukyu Islands, yet has been rarely reported from Japan's main islands. The compactchambered form is distinguished as "var. A" following Ujiié (1985, pl. 1, figs. 9, 10).

Occurrence. Common to abundant in all the formations of the present study. 


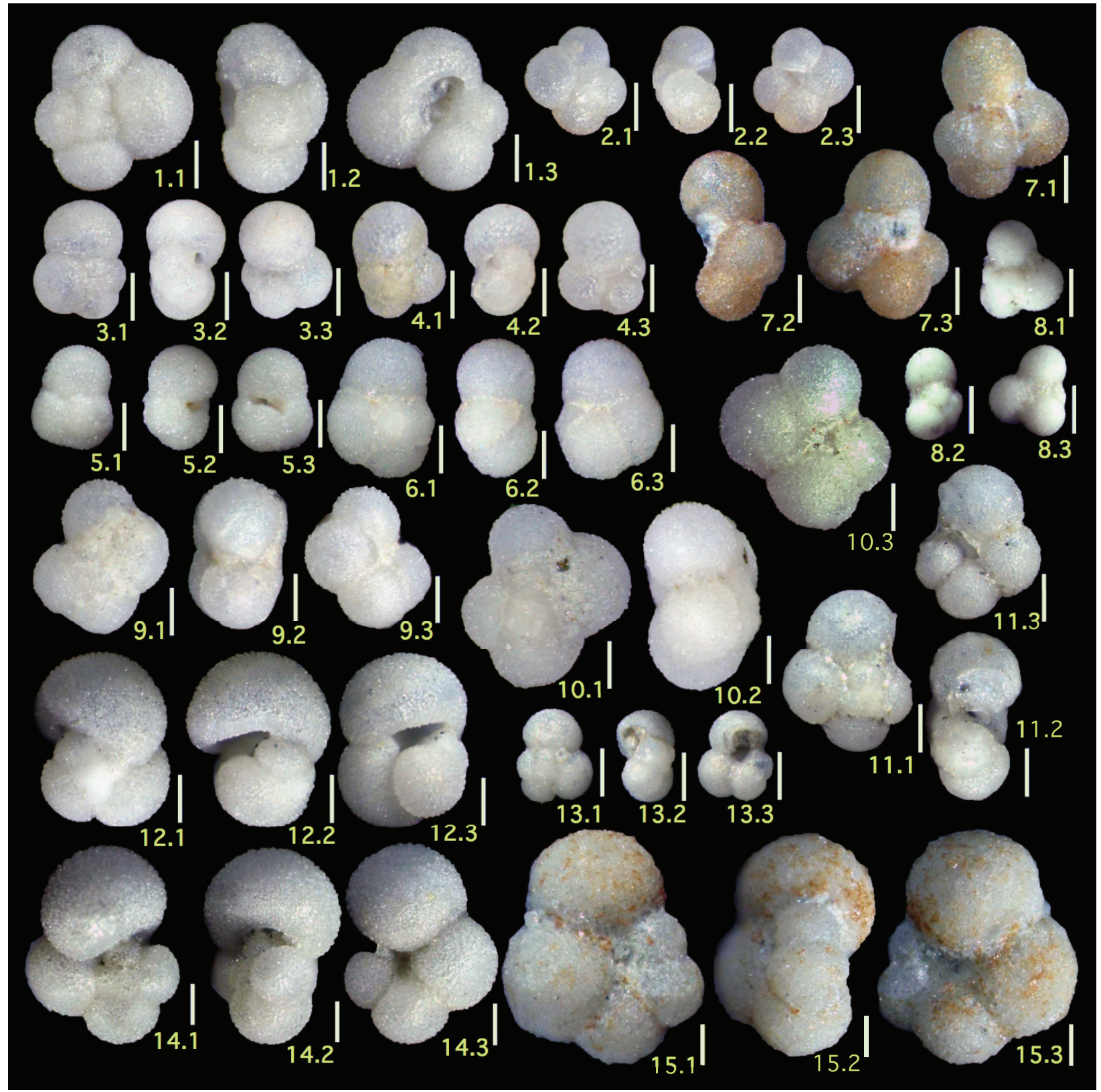

FIGURE 19. Family Globigerinidae. All scale bars $=0.1 \mathrm{~mm} .1$, 2. Globigerina falconensis Blow, 1: MPC-25938 from sample 1 (Minebari Formation, PL5); 2: MPC-25939 from sample MK02B (Minebari F., PL5). 3-6. Globigerina foliata Bolli, 3: MPC-25940 from sample MK01 (Yonahama F., PL2); 4: MPC-25941 from sample MK02B (Minebari F., PL5); 5: MPC-25942 from sample 14 (Yonahama F., PL4); 6: MPC-25943 from sample 16 (Yonahama F., PL3). Specimens of 5 and 6 are morphotype distinguished by Ujiié (1985) as G. foliata var.A. 7, 8. Globigerina bulbosa LeRoy, 7: MPC25934 from sample 4 (Minebari F., PL5); 8: MPC-25935 from sample 7 (Yonahama F., PL4). 9-11. Globigerinella praecalida Blow, 9: MPC-25944 from sample 4 (Minebari F., PL5); 10: MPC-25945 from sample 8-2 (Yonahama F., PL4); 11: MPC-26544 from sample MK01 (Yonahama F., PL2). Last chamber of specimen of 11 lacks last chamber. 12, 13. Globigerinella pseudobesa (Salvatorini), 12: MPC-25941 from sample 5 (Minebari F., PL5); 13: MPC-25947 from sample 11 (Onogoshi F., ?PL1). Specimen of 13 shows a young stage of the species. 14, 15. Globigerinella siphonifera (d'Orbigny), 14: MPC-25948 from sample 1 (Minebari F., PL5); 15: MPC-25949 from sample 13 (Minebari F., PL5). 
Genus GLOBIGERINELLA Cushman, 1927a Globigerinella praecalida (Blow)

Figures 19.9-19.11

1969 Globigerina calida praecalida Blow, p. 380, pl. 13 , figs. 6,7 , pl. 14 , fig. 3 .

1974 Globigerina calida praecalida Blow - Ujiié and Ōki, pl. 1, figs. 1, 2.

1985 Globigerina calida praecalida Blow Ibaraki, pp. 106, 107, pl. 1, figs. 7, 8.

1985 Globigerina praecalida Blow - Ujiié, p. 108, pl. 1, fig. 13, pl. 2, fig. 1.

Remarks. We regard the genus Bolliella Banner and Blow (1959) as a junior synonym of Globigerinella.

Occurrence. Sporadic in the Minebari and Yonahama formations.

Globigerinella pseudobesa (Salvatorini)

Figures 19.12, 19.13

1967 Turborotalia pseudobesa Salvatorini, pp. 666,667 , pl. 2, figs. 6-15, text-fig. 1A.

1977 Globigerina pseudobesa (Salvatorini) Berggren, p. 294, pl. 1, figs. 3-11.

1983 Globigerina pseudobesa (Salvatorini) Kennett and Srinivasan, p. 236, pl. 59, figs. 6 -8.

Occurrence. Rare in the Onogoshi and Minebari formations.

Globigerinella siphonifera (d'Orbigny)

Figures 19.14, 19.15

1839 Globigerina siphonifera d'Orbigny (a), p. 83, pl. 4, figs. 15-18.

1879 Globigerina aequilateralis Brady, p. 285.

1941 Globigerinella aequilateralis (Brady) LeRoy (Part 1), p. 44, pl. 2, figs. 43, 44.

1944 Globigerinella aequilateralis (Brady) LeRoy (Part 1), pp. 40, 41, pl. 3, figs. 24, 25, pl. 6, figs. 37, 38; LeRoy (Part 2), p. 91 , pl. 4, figs. 13, 14.

1959 Globigerinella aequilateralis (Brady) Bradshow, p. 38, pl. 7, figs. 1, 2; Graham and Militante, p. 111, pl. 18, fig. 11.

1959 Globigerinella aequilateralis involute (Cushman) - Blow, pp. 171, 172, pl. 8, fig. 32.

1962 Globigerinella siphonifera (d'Orbigny) Parker, p. 228, pl. 2, figs. 22-28.

1962 Globigerinella aequilateralis aequilateralis (Brady) - Belford, p. 21, pl. 5, figs. 1518.
1964 Globigerinella aequilateralis (Brady) LeRoy, p. F42, pl. 14, figs. 19, 20.

1965 Globigerinella aequilateralis (Brady) Todd, pp. 64, 65, pl. 25, figs. 4, 5.

1967 Globigerinella siphonifera (d'Orbigny) Parker, pp. 152, 153, pl. 22, fig. 5.

1968 Globigerinella siphonifera (d'Orbigny) Huang, p. 60, pl. 11, figs. 16, 17.

1975 Globigerinella siphonifera (d'Orbigny) Ibaraki and Tsuchi, pl. 4, fig. 15.

1977 Globigerinella aequilateralis (Brady) Kadar, p. 67, pl. 4, fig. 26.

1978 Globigerinella aequilateralis (Brady) Oda, p. 52, pl. 2, figs. 8, 9.

1981 Globigerinella aequilateralis (Brady) Saito, Thompson, and Breger, pp. 26, 28, pl. 2, fig. 2.

1985 Globigerinella siphonifera (d'Orbigny) Ibaraki, p. 121, pl. 6, fig. 3.

1985 Globigerinella aequilateralis (Brady) Ujiié, pl. 17, figs. 7, 8.

1985 Hastigerina siphonifera (d'Orbigny) - Bolli and Saunders, pp. 251, 253, figs. 42.142.4, 43.1, 43.2.

1988 Globigerinella aequilateralis (Brady) Wang, Zhang, Zhao, Min, Bian, Zheng, Cheng, and Chen, p. 39, pl. 5, figs. 3, 4.

1994 Globigerinella siphonifera (d'Orbigny) Loeblich and Tappan, p. 106, pl. 200, figs. 7-10, pl. 201, figs. $1-3$.

2004 Globigerinella siphonifera (d'Orbigny) Hanagata, pl. 4, fig. 7.

Remarks. We include the morphotype of $G$. aequilateralis in the present species, although these forms have been differentiated in numerous previous reports.

Occurrence. Rare in the Onogoshi Formation, common in the Yonahama and Minebari formations.

Genus GLOBIGERINOIDES Cushman, 1927a

Globigerinoides extremus Bolli and Bermúdez

Figures 20.1, 20.2

1965 Globigerinoides obliquus extremus Bolli and Bermudez, p. 139, pl. 1, figs. 10-12.

1968 Globigerinoides obliquus Bolli - Huang, p. 60 , pl. 11, figs. 18, 19.

1975 Globigerinoides obliquus extremus Bolli and Bermudez - Ibaraki and Tsuchi, pl. 12, fig. 2. 
1977 Globigerinoides obliquus extremus Bolli and Bermudez - Kadar, p. 67, pl. 1, fig. 7.

1978 Globigerinoides extremus Bolli and Bermudez - Oda, p. 53, pl. 3, figs. 5, 6 .

1983 Globigerinoides extremus Bolli and Bermudez - Kennett and Srinivasan, p. 58, pl. 12, figs. $1-3$.

1985 Globigerinoides extremus Bolli and Bermudez - Ujiié, p. 110, pl. 5, figs. 2-5.

1985 Globigerinoides obliquus extremus Bolli and Bermudez - Ibaraki, p. 113, pl. 3, figs. 13, 14; Bolli and Saunders, pp. 194, 195, fig. 20.11.

2004 Globigerinoides obliquus Bolli - Hanagata, pl. 5 , fig. 5 .

Occurrence. Common in the Oura, Onogoshi, and Yonahama formations; a single specimen from the Minebari Formation.

\section{Globigerinoides obliquus Bolli}

Figures 20.3, 20.4

1957 Globigerinoides obliqua Bolli, p. 113, pl. 25, figs. 9, 10, text-fig. 21.5.

1959 Globigerinoides obliqua Bolli - Blow, pp. 191, 192, pl. 11, fig. 68.

1962 Globigerinoides obliquus Bolli - Belford, p. 20, pl. 5, figs. 11-14.

1967 Globigerinoides obliquus Bolli - Parker, p. 155, pl. 20, fig. 5 (non fig. 6).

1974 Globigerinoides obliquus Bolli - Ujiié and Hatta, pl. 2, figs. 3, 4.

1975 Globigerinoides obliquus obliquus Bolli Ibaraki and Tsuchi, pl. 2, fig. 5.

1977 Globigerinoides obliquus obliquus Bolli Kadar, p. 67, pl. 1, fig. 4.

1978 Globigerinoides obliquus Bolli - Oda, p. 53, pl. 3, figs. 7-9.

1981 Globigerinoides obliquus Bolli - Saito, Thompson, and Breger, p. 54, pl. 13, fig. 3.

1983 Globigerinoides obliquus Bolli - Kennett and Srinivasan, p. 56, pl. 11, figs. 7-9.

1985 Globigerinoides obliquus Bolli — Ujiié, p. 110, pl. 5, figs. 2-5.

1985 Globigerinoides obliquus obliquus Bolli Ibaraki, p. 113, pl. 3, figs. 15, 16; Bolli and Saunders, p. 194, fig. 20.12.

Occurrence. Common in the Oura and Onogoshi formations and the lower part of the Yonahama Formation.
Globigerinoides pyramidalis (Van den Broeck) Figures 20.5, 20.6

1876 Globigerina bulloides d'Orbigny, var. rubra d'Orbigny subvar. pyramidalis Van den Broeck, p. 127, pl. 3, figs. 9, 10.

1941 Globigerinoides rubra (D'Orbigny) LeRoy (Part 3), p. 118, pl. 3, figs. 4-6.

1962 Globigerinoides ruber (d'Orbigny) Parker, pp. 230, 232, pl. 3, figs. 12-14, pl. 4, figs. 1-10; Belford, pl. 5, figs. 4-6 (only).

1964 Globigerinoides ruber (d'Orbigny) LeRoy, p. F42, pl. 14, fig. 14.

1967 Globigerinoides ruber (d'Orbigny) Parker, p. 156, pl. 22, figs. 1-4.

1981 Globigerinoides pyramidalis (van den Broeck) - Saito, Thompson, and Breger, $p$. 62, pl. 16, fig. 1.

1985 Globigerinoides pyramidalis (van den Broeck) - Ujiié, p. 110, pl. 6, figs. 2, 3.

1994 Globigerinoides pyramidalis (van den Broeck) - Jones, p. 89, pl. 79, figs. 13-15; Loeblich and Tappan, p. 107, pl. 204, figs. 4-8, pl. 206, figs. 7-9.

2004 Globigerinoides pyramidalis (van den Broeck) - Hanagata, pl. 5, fig. 6 .

Remarks. This species has a much higher trochospiral test than $G$. ruber which many authors have regarded as a synonym.

Occurrence. Rare in the Onogoshi and Yonahama formations, common in the Minebari Formation.

\section{Globigerinoides ruber (d'Orbigny)}

Figures 20.7-20.9

1839 Globigerina rubra d'Orbigny (a), p. 82, pl. 4, figs. 12-14.

1941 Globigerinoides rubra (d'Orbigny) LeRoy (Part 2), pp. 86, 87, pl. 7, figs. 1315.

1959 Globigerinoides rubra (d'Orbigny) - Bradshow, p. 42, pl. 7, figs. 12, 13; Blow, pp. 192, 193, pl. 11, fig. 70, pl. 13, fig. 69.

1959 Globigerinoides ruber (Orbigny) - Graham and Militante, pp. 111, 112, pl. 18, fig. 12.

1962 Globigerinoides ruber (d'Orbigny) Parker, pp. 230, 232, pl. 3, fig. 11; Belford, pp. 19, 20, pl. 5, figs. 1-3 (only).

1963 Globigerinoides ruber ruber (d'Orbigny) Saito, p. 197, pl. 56, fig. 9. 


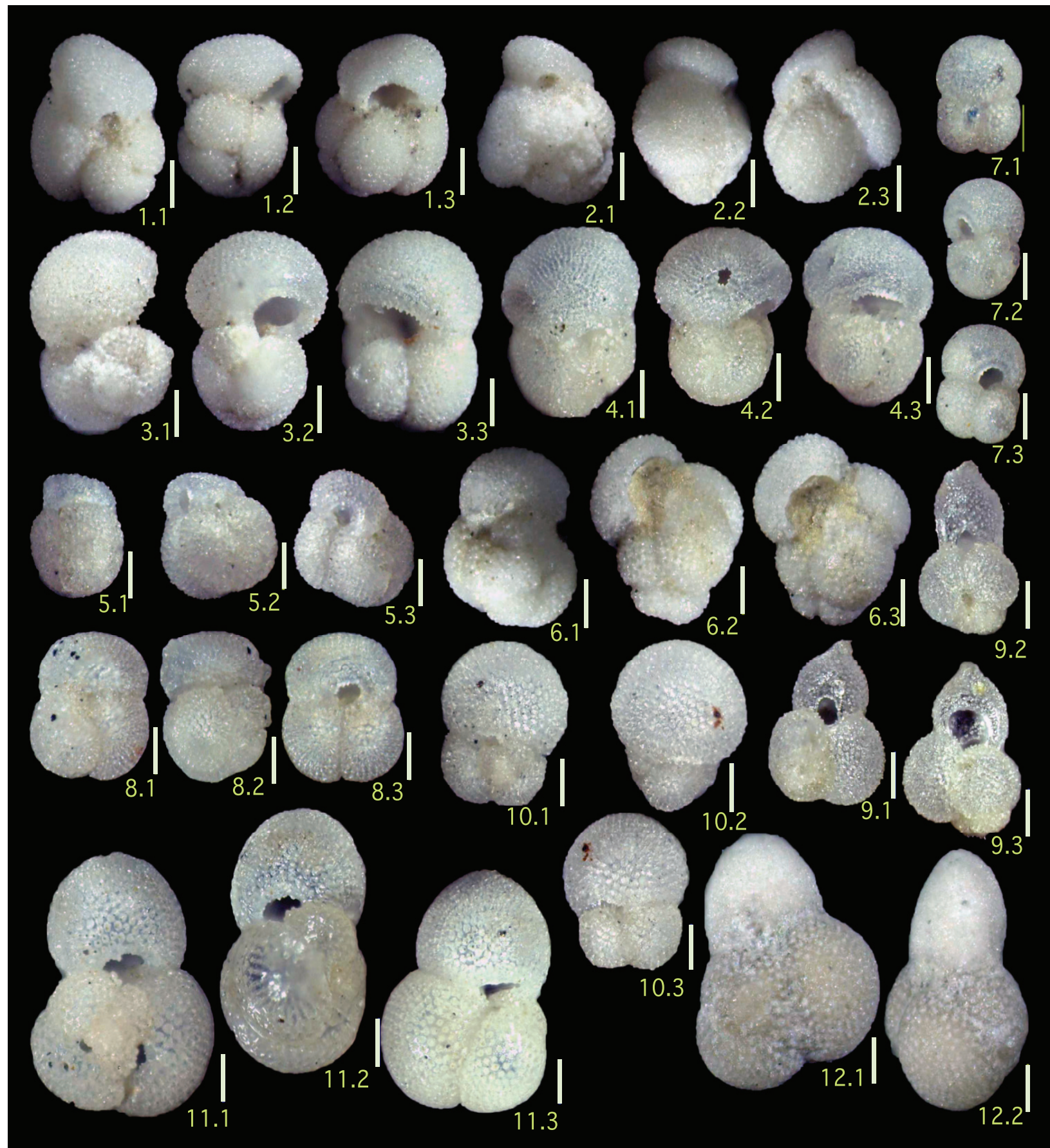

FIGURE 20. Family Globigerinidae. All scale bars $=0.1 \mathrm{~mm} .1$, 2. Globigerinoides extremus Bolli and Bermúdez, 1 : MPC-25955 from sample 14 (Yonahama Formation, PL4); 2: MPC-25956 from sample 15 (Yonahama F., PL3). 3, 4. Globigerinoides obliquus Bolli, 3: MPC-25957 from sample 6 (Yonahama F., PL2); 4: MPC-25958 from sample MK01 (Yonahama F., PL2). 5, 6. Globigerinoides pyramidalis (Van den Broeck), 5: MPC-25959 from sample 1 (Minebari F., PL5); 6: MPC-25960 from sample 2 (Minebari F., PL5). 7-9. Globigerinoides ruber (d'Orbigny), 7: MPC-25961 from sample MK01 (Yonahama F., PL2); 8: MPC-25962 from sample MK02B (Minebari F., PL5); 9: MPC-25963 from sample 1-2 (Minebari F., PL5). Specimen of 9 has aberrant sac-like last chamber. 10-12. Globigerinoides sacculifer (Brady), 10: MPC-25964 and 11: MPC-25965 from sample MK01 (Yonahama F., PL2); 12: MPC-25966 from sample MK02A (Minebari F., PL5). Specimen of 10, young form assignable to G. immatura LeRoy; Specimen of 12 preserves sac-like last chamber. 
1965 Globigerinoides ruber (d'Orbigny) - Todd, p. 63 , pl. 25, fig. 6 .

1967 Globigerinoides ruber ruber (d'Orbigny) Huang, pp. 187, 188, pl. 16, figs. 24, 25 , 27.

1968 Globigerinoides ruber (d'Orbigny) Huang, p. 60, pl. 11, fig. 5.

1975 Globigerinoides ruber (d'Orbigny) Ibaraki and Tsuchi, pl. 3, fig. 6.

1977 Globigerinoides rubra (d'Orbigny) McCulloch, pp. 418, 419, pl. 173, fig. 4.

1977 Globigerinoides ruber (d'Orbigny) Kadar, p. 67, pl. 2, fig. 8.

1978 Globigerinoides ruber (d'Orbigny) - Oda, p. 54, pl. 2, figs. 18, 19.

1981 Globigerinoides ruber (d'Orbigny) - Saito, Thompson, and Breger, p. 59, pl. 15, fig. 1.

1983 Globigerinoides ruber (d'Orbigny) - Kennett and Srinivasan, p. 78, pl. 10, fig. 6, pl. 17 , figs. $1-3$.

1985 Globigerinoides ruber (d'Orbigny) Ibaraki, p. 115, pl. 4, figs. 5, 6; Bolli and Saunders, p. 196, figs. 20.1, 20.2, 20.6.

1988 Globigerinoides ruber (d'Orbigny) Wang, Zhang, Zhao, Min, Bian, Zheng, Cheng, and Chen, pp. 41, 42, pl. 5, figs. 11-14.

1994 Globigerinoides ruber (d'Orbigny) Jones, p. 89 , pl. 79 , figs. $11,12,16$, pl. 81 , figs. 4, 5; Loeblich and Tappan, p. 107, pl. 203 , figs. $1-9$, pl. 206, figs. $10-12$.

2004 Globigerinoides ruber (d'Orbigny) Hanagata, pl. 5, fig. 7.

Occurrence. Common to abundant in all the formations of the present study.

\section{Globigerinoides sacculifer (Brady)}

Figures 20.10-20.12

1884 Globigerina sacculifera Brady, p. 604, pl. 80 , figs. $11-17$, pl. 82, fig. 4.

1941 Globigerinoides sacculiferus (Brady) LeRoy (Part 1), p. 44, pl. 2, figs. 68-70; LeRoy (Part 2), p. 97, pl. 4, figs. 25-27; LeRoy (Part 3), p. 118, pl. 3, figs. 19-21.

1941 Globigerinoides sacculiferus (Brady) var. immature LeRoy - LeRoy (Part 2), p. 87, pl. 7, figs. 16-18.

1944 Globigerinoides sacculiferus (Brady) LeRoy (Part 1), p. 40, pl. 3, figs. 40, 41.

1959 Globigerinoides sacculifers (Brady) Bradshow, p. 42, pl. 7, figs. 14, 15, 18.
1959 Globigerinoides triloba sacculifera (Brady) - Blow, pp. 188, 189, pl. 11, fig. 63.

1959 Globigerinoides sacculifer (Brady) - Graham and Militante, pp. 112, 113, pl. 19, fig. 1.

1962 Globigerinoides quadrilobatus sacculifer (Brady) - Parker, pp. 229, 230, pl. 3, figs. 6-10; Belford, p. 15, pl. 3, figs. 14-16, pl. 4, figs. $1-6$.

1962 Globigerinoides quadrilobatus quadrilobatus (d'Orbigny) - Belford, pp. 12, 13, pl. 2, figs. $17-21$.

1962 Globigerinoides quadrilobatus immaturus Le Roy - Belford, p. 13, pl. 2, figs. 2224, pl. 3, figs. $1-4$.

1962 Globigerinoides quadrilobatus trilobus (Reuss) - Belford, pp. 13, 14, pl. 3, figs. 5-8.

1962 Globigerinoides quadrilobatus irregularis Le Roy - Belford, pp. 14, 15, pl. 3, figs. 9-13.

1963 Globigerinoides trilobus (Reuss) - Saito, p. 199, pl. 54, fig. 15.

1964 Globigerinoides triloba immatua LeRoy LeRoy, p. F42, pl. 14, fig. 16.

1964 Globigerinoides triloba sacculifera (Brady) — LeRoy, p. F42, pl. 14, fig. 18.

1965 Globigerinoides sacculifer (Brady) - Todd, p. 63 , pl. 26, fig. 4 .

1967 Globigerinoides quadrilobatus sacculifer (Brady) - Huang, p. 187, pl. 16, figs. 22, 29.

1967 Globigerinoides sacculifer (Brady) Parker, pp. 156-159, pl. 21, figs. 1, 2, 4, text-fig. 5.

1968 Globigerinoides sacculifer (Brady) Huang, p. 60, pl. 11, figs. 21-23, 29-31.

1975 Globigerinoides quadrilobatus trilobus (Reuss) - Ibaraki and Tsuchi, pl. 2, fig. 3.

1975 Globigerinoides quadrilobatus sacculifer (Brady) - Ibaraki and Tsuchi, pl. 2, fig. 4.

1977 Globigerinoides sacculifera (Brady) McCulloch, p. 419, pl. 173, figs. 8, 10.

1977 Globigerinoides trilobus trilobus (Reuss) Kadar, p. 66, pl. 1, fig. 1.

1977 Globigerinoides trilobus immaturus (LeRoy) - Kadar, p. 66, pl. 1, fig. 2.

1977 Globigerinoides sacculiferus (Brady) Kadar, p. 66, pl. 1, fig. 3. 
1978 Globigerinoides quadrilobatus immaturus LeRoy - Oda, p. 53, pl. 2, figs. 14, 15.

1978 Globigerinoides quadrilobatus sacculifer (Brady) - Oda, pp. 53, 54, pl. 3, figs. 13.

1978 Globigerinoides quadrilobatus trilobus (Reuss) - Oda, p. 54, pl. 2, fig. 11.

1981 Globigerinoides sacculifer (Brady) Saito, Thompson, and Breger, pp. 65, 66, pl. 17, figs. 1, 2.

1983 Globigerinoides triloba (Brady) - Kennett and Srinivasan, p. 62, pl. 10, fig. 4, pl. 13, figs. $1-3$.

1983 Globigerinoides imaturus LeRoy - Kennett and Srinivasan, p. 64, pl. 10, fig. 3, pl. 13, figs. 7-9.

1983 Globigerinoides quadrilobatus (Brady) Kennett and Srinivasan, p. 66 , pl. 14, figs. $1-3$.

1983 Globigerinoides sacculifer (Brady) - Kennett and Srinivasan, p. 66 , pl. 14, figs. 46.

1985 Globigerinoides quadrilobatus immaturus LeRoy - Ibaraki, pp. 113, 114, pl. 3, figs. 17, 18.

1985 Globigerinoides quadrilobatus sacculifer (Brady) - lbaraki, p. 114, pl. 4, figs. 1, 2.

1985 Globigerinoides quadrilobatus trilobus (Reuss) - Ibaraki, pp. 114, 115, pl. 4, figs. 3, 4.

1985 Globigerinoides quadrilobatus sacculifer (Brady) - Ujiié, pl. 4, figs. 5, 6.

1985 Globigerinoides trilobus sacculifer (Brady) — Bolli and Saunders, p. 196, fig. 20.13.

1988 Globigerinoides sacculifer (Brady) Wang, Zhang, Zhao, Min, Bian, Zheng, Cheng, and Chen, p. 42, pl. 6, figs. 1-12.

1994 Globigerinoides sacculifer (Brady) Jones, p. 89 , pl. 80 , figs. $11-17$, pl. 81 , figs. 2, 3, pl. 82, fig. 4.

1994 Globigerinoides sacculifers (Brady) Loeblich and Tappan, p. 107, pl. 205, figs. $1-9$.

1994 Globigerinoides trilobus (Reuss) - Loeblich and Tappan, p. 107, pl. 206, figs. 16.

2004 Globigerinoides sacculifer (Brady) Hanagata, pl. 5, fig. 8, pl. 6, fig. 1.

Remarks. Bé (1980) summarized synonyms of this species, and we here follow his views (see synon- ymy). André et al. (2013) confirmed such morphological variation in this species on the basis of molecular evidence.

Occurrence. Common to abundant in all the formations of the present study.

Genus GLOBIGERINOIDESELLA El-Naggar, 1971 Globigerinoidesella fistulosa (Schubert)

Figure 21.1

1910 Globigerina fistulosa Schubert, pp. 323, 324, text-fig. 2, fig. 13.

1962 Globigerinoides quadrilobatus fistulosus (Schubert) - Belford, pp. 16, 17, pl. 4, figs. 7-10.

1964 Globigerinoides triloba fistulosa (Schubert) — LeRoy, p. F42, pl. 14, fig. 17.

1967 Globigerinoides fistulosus (Schubert) Parker, pp. 154, 155, pl. 21, figs. 3, 5, 6, text-fig. 4.

1967 Globigerinoides quadrilobatus fistulosus (Schubert) - Huang, p. 187, pl. 16, fig. 28.

1968 Globigerinoides fistulosa (Schubert) Huang, p. 60, pl. 11, fig. 28.

1977 Globigerinoides fistulosa (Schubert) McCulloch, p. 418, pl. 173, fig. 11.

1981 Globigerinoides fistulosa (Schubert) Saito, Thompson, and Breger, p. 68, pl. 18, figs. 1, 2.

1983 Globigerinoides fistulosa (Schubert) Kennett and Srinivasan, p. 68, pl. 14, figs. 7-9.

1985 Globigerinoides fistulosa (Schubert) Ujiié, p. 110, pl. 4, fig. 7, pl. 5, fig. 1.

1985 Globigerinoides trilobus fistulosus (Schubert) - Bolli and Saunders, pp. 196-198, figs. 22.5-22.11.

1987 Globigerinoidesella fistulosa (Schubert) Loeblich and Tappan, pl. 536, figs. 7, 8.

2004 Globigerinoidesella fistulosa (Schubert) Hanagata, pl. 3, fig. 3.

Occurrence. Three specimens from a single sample of the Yonahama Formation.

Genus GLOBOTURBOROTALITA Hofker 1976

Globoturborotalita decoraperta (Takayanagi and Saito)

Figures 21.2, 21.3

1962 Globigerina druyi Akers decoraperta Takayanagi and Saito, p. 85, pl. 28, fig. 10.

1967 Globigerina decoraperta Takayanagi and Saito - Parker, pp. 149, 150, pl. 19, figs. $1,2$. 
1974 Globigerina decoraperta Takayanagi and Saito - Ujiié and Ōki, pl. 1, fig. 5.

1978 Globigerina decoraperta Takayanagi and Saito - Oda, p. 50, pl. 1, figs. 4-6.

1983 Globigerina (Zeaglobigerina) decoraperta Takayanagi and Saito - Kennett and Srinivasan, p. 48, pl. 9, figs. 4-6.

1985 Globigerina decoraperta Takayanagi and Saito - Ujiié, p. 109, pl. 2, figs. 5, 6; Ibaraki, p. 107, pl. 1, figs. 9, 10; laccarino, p. 302, figs. 5.5 .

2004 Globoturborotalita decoraperta (Takayanagi and Saito) - Hanagata, pl. 6, fig. 4.

Occurrence. Common in all the formations of the present study.

Globoturborotalita nepenthes (Todd)

Figures 21.4, 21.5

1957 Globigerina nepenthes Todd, p. 301, pl. 78, fig. 7.

1959 Globigerina nepenthes Todd - Blow, pp. 178, 179, pl. 8, figs. 44, 45.

1967 Globigerina nepenthes Todd - Parker, pp. 150 , 151, pl. 19, fig. 10; Huang, p. 187, pl. 16 , figs. $7,13,14$.

1977 Globigerina nepenthes Todd - Kadar, p. 63, pl. 2, fig. 11.

1978 Globigerina nepenthes Todd - Oda, p. 51, pl. 1, figs. 12-14; Keller(a), pl. 5, figs. 12, 13.

1983 Globigerina (Zeaglobigerina) nepenthes Todd - Kennett and Srinivasan, p. 48, pl. 9, figs. $1-3$.

1985 Globigerina nepenthes Todd - Ujiié, p. 109, pl. 2, figs. 3, 4; Ibaraki, p. 108 , pl. 2 , figs. 3, 4; Bolli and Saunders, pp. 201, 202, fig. 25.

Remarks. Specimens from Miyakojima Island illustrate the young stage; the development of a high arched aperture which characterizes this species is not observed. The vertically growing aperture that is accompanied by a distinct rim discriminates this species from congeners.

Occurrence. Common in the Oura and Onogoshi formations.

\section{Globoturborotalita rubescens (Hofker)}

Figures 21.6, 21.7

1956 Globigerina rubescens Hofker (b), p. 234, pl. 32, fig. 26, pl. 35, figs. 18-21.

1962 Globigerina rubescens Hofker - Parker, p. 226, pl. 2, figs. 17, 18.
1967 Globigerina rubescens Hofker - Parker, p. 152, pl. 19, figs. 3, 4.

1968 Globigerina riveroae Bolli and Bermudez - Huang, p. 60, pl. 11, fig. 4 (non G. riveroae Bolli and Bermudez, 1965).

1973 Globigerina (Zeaglobigerina) rubescens Hofker - Kennett and Srinivasan, p. 50, pl. 99, figs. 7-9.

1974 Globigerina rubescens Hofker - Ujiié and Ōki, pl. 1, fig. 4.

1977 Globigerina rubescens Hofker — Kadar, p. 61 , pl. 2, fig. 9.

1981 Globigerina rubescens Hofker - Saito, Thompson, and Breger, p. 50, pl. 11, fig. 1.

1985 Globigerina rubescens Hofker - Ujiié, p. 109 , pl. 2, fig. 7; Ibaraki, p. 110, pl. 2, figs. $15,16$.

1987 Globoturborotalita rubescens (Hofker) Loeblich and Tappan, pl. 537, figs. 7-15.

1988 Globigerina rubescens Hofker - Wang, Zhang, Zhao, Min, Bian, Zheng, Cheng and Chen, pp. 40, 41, pl. 7, figs. 16-17

1994 Globoturborotalita rubescens (Hofker) Loeblich and Tappan, p. 108, pl. 208, figs. $1-12$.

2004 Globoturborotalita rubescens (Hofker) Hanagata, pl. 6, fig. 5.

Occurrence. Common to abundant in all the formations of the present study.

Genus SPHAEROIDINELLA Cushman, 1927a

Sphaeroidinella dehiscens (Parker and Jones)

Figures 21.8, 21.9

1865 Sphaeroidina bulloides d'Orbigny var. dehiscens Parker and Jones, p. 369, pl. 19, fig. 5.

1941 Sphaeroidinella dehiscens (Parker and Jones) - p. 87, pl. 6, fig. 13.

1959 Sphaeroidinella dehiscens (Parker and Jones) - Bradshow, p. 49, pl. 8, figs. 2123; Graham and Militante, p. 114, pl. 19, fig. 5 .

1962 Sphaeroidinella dehiscens dehiscens (Parker and Jones) - Belford, p. 30, pl. 8, figs. 19-21.

1964 Sphaeroidinella dehiscens (Parker and Jones) - LeRoy, p. F43, pl. 14, figs. 19, 20.

1967 Sphaeroidinella dehiscens (Parker and Jones) - Parker, pp. 160, 161, pl. 23, figs. $8,9$. 


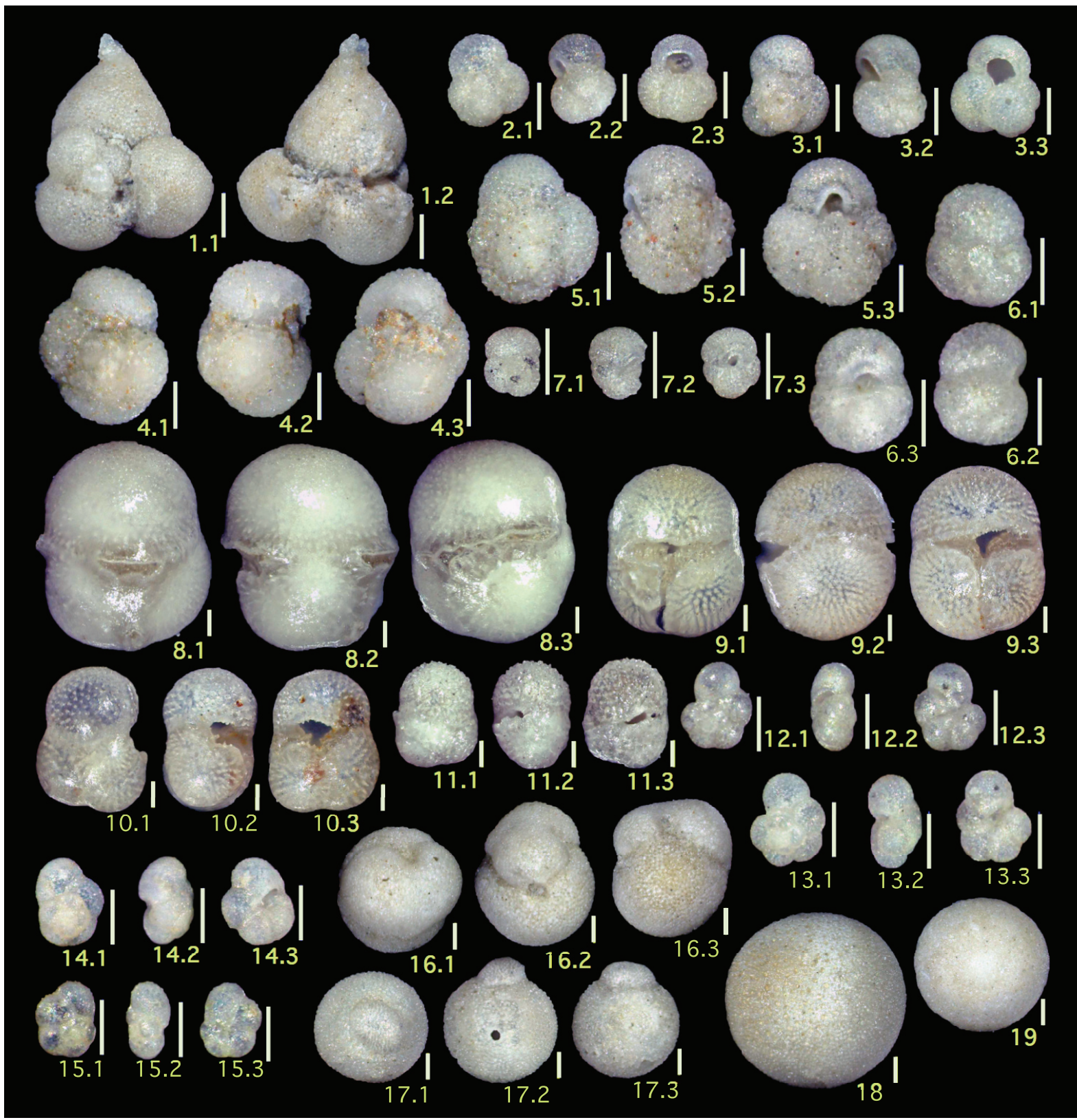

FIGURE 21. Family Globigerinidae, All scale bars $=0.1 \mathrm{~mm}$. 1. Globigerinoidesella fistulosa (Schubert), MPC-25967 from sample 8-2 (Yonahama Formation, PL4). Poor preservation is due to serious recrystallization. 2, 3. Globoturborotalita decoraperta (Takayanagi and Saito), 2: MPC-25990 from sample 6 (Yonahama F., PL2); 3: MPC-25991 from sample MK01 (Yonahama F., PL2). 4, 5. Globoturborotalita nepenthes (Todd), 4: MPC-25992 from sample 3 (Onogoshi F., PL1); 5: MPC-25993 from sample 11 (Onogoshi F., ?PL1). Specimens of this study are young form lack elongate later chambers, but narrow-raising aperture with distinct rim characterize this species. 6, 7. Globoturborotalita rubescens (Hofker), 6: MPC-25994 from sample 6 (Yonahama F., PL2); 7: MPC-25995 from sample MK01 (Yonahama F., PL2). 8, 9. Sphaeroidinella dehiscens (Parker and Jones), 8: MPC-26025 from sample 8-2 (Yonahama F., PL4); 9: MPC-26026 from sample MK02B (Minebari F., PL5). 10, 11. Sphaeroidinellopsis seminulina (Schwager), 10: MPC-26027 from sample MK01 (Yonahama F., PL2); 11: MPC-26028 from sample 6 (Yonahama F., PL2). 12, 13. Turborotalita humilis (Brady), 12: MPC-26029 from sample 10 (Onogoshi F., PL2); 13: MPC-26030 from sample MK01 (Yonahama F., PL2). 14, 15. Turborotalita quinqueloba (Natland), 14: MPC-26031 from sample MK01 (Yonahama F., PL2); 15: MPC-26032 from sample MK02B (Minebari F., PL5). 16, 17. Orbulina suturalis Brönnimann, 16: MPC26015 from sample 8 (Yonahama F., PL4); 17: MPC-26016 from sample 14 (Yonahama F., PL4). 18, 19. Orbulina universa d'Orbigny, 18: MPC-26017 from sample 8-2 (Yonahama F., PL4); 19: MPC-26018 from sample MK02A (Minebari F., PL5). 
1967 Sphaeroidinella dehiscens dehiscens (Parker and Jones) - Huang, p. 188, pl. 16 , figs. 12, 31, 32, 34, 36.

1976 Sphaeroidinella dehiscens dehiscens (Parker and Jones) - Natori, pl. 1, fig. 6.

1977 Sphaeroidinella dehiscens (Parker and Jones) - McCulloch, p. 407, pl. 174, figs. 17, 18; Kadar, p. 65, pl. 3, fig. 19.

1978 Sphaeroidinella dehiscens (Parker and Jones) - Oda, p. 64, pl. 10, figs. 16, 17.

1981 Sphaeroidinella dehiscens (Parker and Jones) - Saito, Thompson, and Breger, pp. 72, 74, pl. 20, fig. 2.

1983 Sphaeroidinella dehiscens (Parker and Jones) - Kennett and Srinivasan, p. 212, pl. 51, fig. 2, pl. 52, figs. 7-9.

1985 Sphaeroidinella dehiscens (Parker and Jones) - Ujiié, p. 111, pl. 8, fig. 2; Bolli and Saunders, pp. 244, 245, figs. 39.139.8 .

1985 Sphaeroidinella dehiscens dehiscens (Parker and Jones) - Ibaraki, p. 139, pl. 13, figs. 9, 10.

1988 Sphaeroidinella dehiscens (Parker and Jones) - Wang, Zhang, Zhao, Min, Bian, Zheng, Cheng, and Chen, p. 44, pl. 5, figs. 7-9.

1994 Sphaeroidinella dehiscens (Parker and Jones) - Jones, pp. 91, 92, pl. 84, figs. 8-11.

2004 Sphaeroidinella dehiscens (Parker and Jones) - Hanagata, pl. 6, fig. 6 .

Remarks. We do not distinguish subspecies of $S$. dehiscens in the present study. Specimens from the sequence studied are primitive in that the opening of encrustation is not broad, so our material include the primitive subspecies $S$. dehiscens immatura (Cushman) (e.g., Natori, 1976, pl. 1, fig. $5)$, while the advanced subspecies $S$. dehiscens excavata Banner and Blow (e.g., Natori, 1976, pl. 1, fig. 7) has not been observed.

Occurrence. Common in the upper part of the Yonahama Formation and the Minebari Formation. Genus SPHAEROIDINELLOPSIS Banner and Blow, 1959

\section{Sphaeroidinellopsis seminulina (Schwager)}

Figures 21.10, 21.11

1866 Globigerina seminula Schwager, p. 256, pl. 7, fig. 112.

1941 Globigerina seminulina Reuss - LeRoy (Part 1), p. 44, pl. 3, fig. 108.
1959 Sphaeroidinella dehiscens subdehiscens Blow, pp. 195, 196, pl. 12, figs. 71, 72.

1959 Sphaeroidinella seminula seminulina (Schwager) - Blow, pp. 197, 198, pl. 12, figs. 74-76.

1962 Sphaeroidinellopsis subdehiscens (Blow) - Belford, pp. 30, 31, pl. 8, figs. 26-28.

1962 Sphaeroidinellopsis seminulina seminulina (Schwager) - Belford, p. 31, pl. 8, figs. 22-25.

1963 Sphaeroidinellopsis seminulina (Schwager) - Saito, pp. 200, 201, pl. 56, fig. 13.

1963 Sphaeroidinellopsis subdehiscens (Blow) — Saito, p. 201, pl. 56, fig. 12.

1964 Sphaeroidinella seminula (Schwager) LeRoy, p. F43, pl. 14, figs. 23, 24.

1967 Sphaeroidinella seminula (Schwager) Parker, pp. 161, 162, pl. 23, figs. 1-5.

1967 Sphaeroidinella subdehiscens Blow Parker, p. 162, pl. 23, figs. 6, 7.

1968 Sphaeroidinellopsis seminulina (Schwager) - Huang, p. 61, pl. 11, figs. 26, 27.

1969 Sphaeroidinellopsis subdehiscens subdehiscens (Blow) - Blow, p. 338, pl. 20, figs. 1-3, 6, pl. 31, figs. 1-3.

1974 Sphaeroidinellopsis seminula seminula (Schwager) - Ujiié and Ōki, pl. 1, fig. 12.

1976 Sphaeroidinellopsis seminula seminula (Schwager) - Natori, p. 229, pl. 1, fig. 2.

1976 Sphaeroidinellopsis subdehiscens subdehiscens (Blow) - Natori, p. 229, pl. 1, fig. 1.

1977 Sphaeroidinella seminulina (Schwager) Kadar, p. 64, pl. 3, figs. 17, 18.

1978 Sphaeroidinellopsis seminulina (Schwager) - Oda, p. 64, pl. 10, figs. 11, 12.

1978 Sphaeroidinella subdehiscens Blow Keller (a), pl. 5, figs. 14, 15.

1983 Sphaeroidinellopsis seminula seminula (Schwager) - Kennett and Srinivasan, pp. 206, 208, pl. 51, figs. 1, 6-8.

1985 Sphaeroidinellopsis seminula seminula (Schwager) - Ibaraki, p. 140, pl. 13, figs. 12, 13.

1985 Sphaeroidinellopsis subdehiscens subdehiscens (Blow) - Ibaraki, p. 141, pl. 13, figs. 16, 17.

1985 Sphaeroidinellopsis seminulina (Schwager) - Ujiié, pp. 110, 111, pl. 7, figs. 9-11; 
Bolli and Saunders, pp. 241, 242, figs. 38.6-38.13.

Remarks. We do not separate the morphotype subdehiscens Blow from S. seminulina following the view expressed by Srinivasan and Kennett (1981).

Occurrence. Low in number, but continuously present from the Onogoshi Formation to the lower part of the Yonahama Formation.

Genus TURBOROTALITA Blow and Banner in

Eames, Banner, Blow, and Clarke, 1962

Turborotalita humilis (Brady)

Figures 21.12, 21.13

1884 Truncatulina humilis Brady, p. 665, pl. 94, fig. 7.

1962 Globigerinita humilis (Brady) - Parker, pp. 249,250 , pl. 10, figs. $1-25$.

1965 Globigerinita humilis (Brady) - Todd, pp. 66,67 , pl. 25, figs. $1,2$.

1967 Turborotalita humilis (Brady) - Parker, pp. 146, 147, pl. 17, fig. 10.

1981 Turborotalita humilis (Brady) - Saito, Thompson, and Breger, pl. 25, figs. 1-3.

1983 Turborotalita humilis (Brady) - Kennett and Srinivasan, p. 167, pl. 41, figs. 1, 3-5.

1985 Turborotalita humilis (Brady) - Ibaraki, p. 123, pl. 6, figs. 16, 17; Bolli and Saunders, p. 188 , fig. 17.9 .

1994 Turborotalita humilis (Brady) - Loeblich and Tappan, p. 109, pl. 210, figs. 1-12.

2003 Turborotalia humilis (Brady) - Ujiié, pp. 41, 42, pl. 1, fig. 4.

2004 Turborotalita humilis (Brady) - Hanagata, pl. 3, fig. 7.

Occurrence. Sporadic in the Onogoshi and Yonahama formations.

Turborotalita quinqueloba (Natland)

Figures 21.14, 21.15

1838 Globigerina quinqueloba Natland, p. 149, pl. 6 , fig. 7.

1959 Globigerina quinqueloba Natland - Bradshow, p. 38, pl. 6, figs. 24, 25.

1962 Globigerina quinqueloba Natland Parker, pp. 225, 226, pl. 2, figs. 7-16.

1967 Globigerina cf. G. quinqueloba Natland Parker, pp. 151, 152, pl. 18, figs. 13, 14.

1978 Globigerina quinqueloba Natland - Oda, pp. 51,52 , pl. 2 , figs. 5,6 .
1981 Globigerina quinqueloba Natland - Saito, Thompson, and Breger, p. 48, pl. 10, figs. $1,2$.

1983 Globigerina quinqueloba Natland - Kennett and Srinivasan, p. 32, pl. 5, figs. 4-6.

1985 Globigerina quinqueloba Natland Ibaraki, p. 110, pl. 2, figs. 13, 14; Jenkins, p. 275 , fig. 7.2 .

1988 Globigerina quinqueloba Natland - Wang, Zhang, Zhao, Min, Bian, Zheng, Cheng and Chen, p. 14, pl. 8, figs. 1-6.

1994 Berggrenia pumilio (Parker) - Loeblich and Tappan, p. 100, pl. 180, figs. 8-13. (not pl. 181, figs. 1-3) (non Globorotalia pumilio Parker, 1962)

2004 Turborotalita quinqueloba (Natland) Hanagata, pl. 3, fig. 6.

Occurrence. Common in the Oura, Onogoshi, and Minebari formations, abundant in the Yonahama Formation.

Subfamily ORBULININAE Schultze, 1854 Genus ORBULINA d'Orbigny, 1839a Orbulina suturalis Brönnimann

Figures 21.16, 21.17

1951 Orbulina suturalis Brönnimann (b), p. 271, pl. 31, fig. 1.

1959 Orbulina suturalis Brönnimann - Blow, p. 200, pl. 13, fig. 69.

1977 Orbulina suturalis Brönnimann — Kadar, $p$. 66, pl. 4, fig. 25.

1978 Orbulina suturalis Brönnimann — Oda, p. 62, pl. 5, fig. 16.

1983 Orbulina suturalis Brönnimann - Kennett and Srinivasan, p. 86, pl. 20, figs. 1-3.

1985 Orbulina suturalis Brönnimann - Ujiié, pl. 6, fig. 7; Ibaraki, pp. 119, 120, pl. 5, fig. 10; Papp and Schmid, p. 20, pl. 1, figs. 1-5; Bolli and Saunders, p. 201, figs. 23.2, 24.3.

2004 Orbulina suturalis Brönnimann - Hanagata, pl. 6, fig. 7.

Occurrence. Rare in the Minebari and Yonahama formations.

Orbulina universa d'Orbigny

Figures 21.18, 21.19

1839 Orbulina universa d'Orbigny (a), p. 3, pl. 1, fig. 1.

1941 Orbulina universa d'Orbigny - LeRoy (Part 1), p. 44, pl. 1, fig. 4; LeRoy (Part 2), 
p. 87, pl. 1, fig. 27; LeRoy (Part 3), p. 118, pl. 1, fig. 17.

1944 Orbulina universa d'Orbigny - LeRoy (Part 1), p. 41, pl. 3, figs. 44, 45; LeRoy (Part 2), p. 91, pl. 3, fig. 19.

1959 Orbulina universa d'Orbigny - Bradshow, p. 49 , pl. 8 , figs. 17,18 ; Blow, p. 200, pl. 13, fig. 83; Graham and Militante, p. 113, pl. 19, fig. 3.

1962 Orbulina universa d'Orbigny - Belford, $p$. 6 -9, pl. 1, figs. 1-15.

1964 Orbulina universa d'Orbigny - LeRoy, p. F42, pl. 14, fig. 5.

1968 Orbulina universa d'Orbigny - Huang, $p$. 61, pl. 10, fig. 15.

1977 Orbulina universa d'Orbigny - Kadar, p. 66, pl. 4, fig. 24.

1983 Orbulina universa d'Orbigny - Kennett and Srinivasan, pp. 86, 88, pl. 18, fig. 2, pl. 20, figs. 4-6.

1985 Orbulina universa d'Orbigny - Ibaraki, p. 120, pl. 5, fig. 11; Bolli and Saunders, p. 201, figs. 23.1, 24.2 .

1988 Orbulina universa d'Orbigny - Wang, Zhang, Zhao, Min, Bian, Zheng, Cheng, and Chen, p. 44, pl. 5, figs. 5, 6 .

1994 Orbulina universa d'Orbigny - Jones, p. 88, pl. 78, figs. 8-27, pl. 82, figs. 1-3; Loeblich and Tappan, p. 109, pl. 207, figs. $1-3$, pl. 211, figs. 4-7.

2004 Orbulina universa d'Orbigny - Hanagata, pl. 6 , fig. 8.

Occurrence. Common in all the formations of the present study.

Suborder ROTALIINA Delage and Hérouard, 1896

Superfamily BOLIVINOIDEA Glaessner, 1937

Family BOLIVINIDAE Glaessner, 1937

Genus BOLIVINA d'Orbigny, 1839c

Bolivina antiqua d'Orbigny

Figures 22.1, 22.2

1846 Bolivina antiqua d'Orbigny, p. 240, pl. 14, figs. 11-13.

1985 Bolivina antiqua d'Orbigny - Papp and Schmid, p. 83, pl. 77, figs. 1-6.

1994 Bolivinellina translucens (Phleger and Parker) - Loeblich and Tappan, p. 111, pl. 213, figs. 9-14 (non Bolivina translucens Phleger and Parker, 1951)

Remarks. Bolivina translucens has more inflated chambers and depressed sutures than the present species. The discrimination between the genera Bolivina and Brizalina has been controversial. Loeblich and Tappan (1987) considered Brizalina to be a valid genus, but Sgrella (1992) restudied Brizalina aenariensis Costa, type species of the genus, and concluded that it had features fitting Bolivina. Loeblich and Tappan (1994) followed Sgrella's (1992) conclusion. Revets (1996a), on the other hand, remarked that Sgrrella's (1992) examination was insufficient and retained the genus Brizalina, putting weight on the apertural character. We do not distinguish Brizalina here, following Loeblich and Tappan (1994), since the inner apertural character does not seem critical in separation taxa in this complex taxonomic group.

Occurrence. Common in the Oura Formation.

Bolivina cacozela Vella

Figures 22.3-22.6

1957 Bolivina cacozela Vella, p. 33, pl. 8, figs. 162, 163.

2001 Bolivina cacozela Vella - Hayward, Carter, Grenfell, and Hayward, p. 583, figs. 14L, 14-M.

Diagnosis. Moderate-sized and elongate species with perforated, yet smooth, flush surface and limbate septa.

Remarks. We recognize small and large forms; the former might be a different species, but we have tentatively included it here on the basis of similar morphology.

Occurrence. Sporadic in the Onogoshi, Yonahama, and Minebari formations. The large form is confined to the Minebari Formation.

\section{Bolivina cochei Cushman and Adams}

Figures 22.17, 22.18

1935 Bolivina cochei Cushman and Adams, p. 19, pl. 3, figs. 6,7 .

Remarks. This species resembles Bolivina robusta in test size and general shape, but is distinguished by its compressed test with acute and thinly carinated periphery. Revets (1996a) suggested that this species belonged to the genus Brizalina, not Bolivina. It also resembles Bolivina marginata Cushman (1918) in possessing a peripheral keel, but that form has a more slender test.

Occurrence. Abundant in a single sample of the Yonahama Formation.

\section{Bolivina glutinata Egger}

Figures 22.7, 22.8

1893 Bolivina glutinata Egger, p. 297, pl. 8, figs. 57-62. 


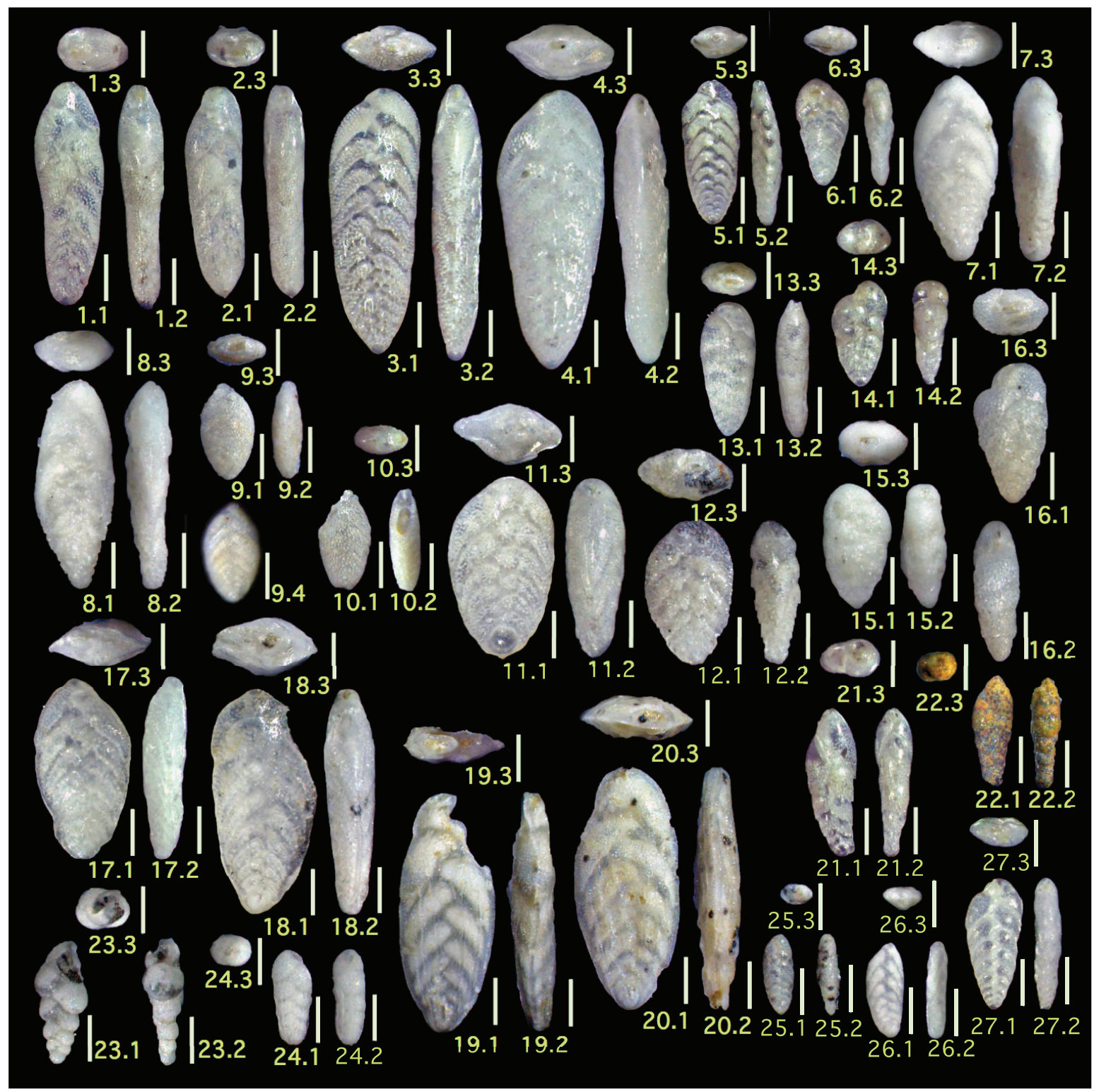

FIGURE 22. Family Bolivinidae. All scale bars $=0.1 \mathrm{~mm} .1$, 2. Bolivina antiqua d'Orbigny, 1: MPC-26140, 2: MPC26141, both from sample 18 (Oura Formation, ?PL1). 3-6. Bolivina cacozela Vella, 3: MPC-26142 from sample 2-2 (Minebari F., PL5); 4: MPC-26143 from sample 5 (Minebari F., PL5); 5: MPC-26144 from sample 10 (Onogoshi F., PL2); 6: MPC-26145 from sample 16 (Yonahama F., PL3). Specimens of 3 and 4 have larger test size than those of 5 and 6. 7, 8. Bolivina glutinata Egger, 7: MPC-26148 from sample 15 (Yonahama F., PL3); 8: MPC-26149 from sample 16 (Yonahama F., PL3). 9, 10. Bolivina retia Oki, 9: MPC-26154, 10: MPC-26545, both from sample 4 (Minebari F., PL5); 9.4, moisturized specimen showing internal septa. 11, 12. Bolivina robusta Brady, 11: MPC-26155 from sample 5 (Minebari F., PL5); 12: MPC-26156 from sample 10 (Onogoshi F., PL2). 13, 14. Bolivina striatula Cushman, 13: MPC-26157 from sample 14-2 (Yonahama F., PL4); 14: MPC-26158 from sample MK01 (Yonahama F., PL2). 15, 16. Bolivina variabilis (Williamson), 15: MPC-26161 from sample 16 (Yonahama F., PL3); 16: MPC-26162 from sample MK01 (Yonahama F., PL2). 17, 18. Bolivina cochei Cushman and Adams, 17: MPC-26146, 18: MPC-26147, both from sample MK01 (Yonahama F., PL2). 19, 20. Bolivina kiiensis Asano, 19: MPC-26150 from sample 3 (Onogoshi F., PL1); 20: MPC-26151 from sample 3-2-low-silt (Onogoshi F., PL1). 21, 22. Bolivina pacifica Cushman and McCulloch, 21: MPC-26152 from sample 1-2 (Minebari F., PL5); 22: MPC-26153 from sample MK01 (Yonahama F., PL2). 23. Bolivina cf. subspinescens Cushman, MPC-26159 from sample MK01 (Yonahama F., PL2). 24. Bolivina cf. yabei Takayanagi, MPC-26163 from sample MK01 (Yonahama F., PL2). 25, 26. Bolivina sp.A, 25: MPC-26164 from sample 11 (Onogoshi F., ?PL1); 26: MPC-26165 from sample 16 (Yonahama F., PL3). 27. Bolivina trans/ucens Phleger and Parker, MPC-26160 from sample 5-2 (Minebari F., PL5). 
1994 Bolivina glutinata Egger - Loeblich and Tappan, p. 111, pl. 213, figs. 1-8.

Diagnosis. Moderate-sized species with thick and roughly developed surface wall, and strongly curved sutures towards the periphery.

Occurrence. Sporadic in the Yonahama and Minebari formations.

Bolivina kiiensis Asano

Figures 22.19, 22.20

1958 Bolivina kiiensis Asano, pp. 19, 20, pl. 4, figs. 7, 8.

1989 Bolivina kiiensis Asano - Ōki, pl. 9, fig. 4; Inoue, pl. 32, fig. 11.

Remarks. Some specimens show that the aperture is slightly separately bordered from the attaching point with the penultimate chamber by a marginal ridge, so the generic assignment of this species might have to be changed on the basis of additional observations of well-preserved specimens.

Occurrence. Common in the Onogoshi Formation, rare in the Minebari Formation.

Bolivina pacifica Cushman and McCulloch

Figures 22.21, 22.22

1942 Bolivina acerosa Cushman var. pacifica Cushman and McCulloch, p. 185, pl. 21, figs. 2, 3.

1989 Bolivina pacifica Cushman and McCulloch - Ōki, p. 109, pl. 9, fig. 6; Inoue, pl. 32, fig. 4, pl. 33, fig. 16.

1990 Brizalina pacifica (Cushman and McCulloch) - Akimoto, p. 193, pl. 16, fig. 16.

Occurrence. Sporadic in the Onogoshi, Yonahama, and Minebari formations.

Bolivina retia Ōki

Figures 22.9, 22.10

1989 Bolivina retia Ōki, pp. 109, 110, 170, pl. 9, fig. 7.

1995 Bolivina retia Ōki - Ujiié, p. 60, pl. 4, figs. 2, 3.

Diagnosis. Small-sized species with fine reticulation on test surface.

Remarks. This species was originally recorded from Kagoshima Bay, southwest Japan, and probably was a shallow-water, endemic form around the Ryukyu Island Arc and southwest Japan.

Occurrence. Two specimens from a single sample of the Minebari Formation.

Bolivina robusta Brady

Figures 22.11, 22.12

1884 Bolivina robusta Brady, p. 421, pl. 53, figs. 7-9.
1911 Bolivina robusta Brady - Cushman, pp. 36, 37, figs. 59, 60.

1941 Bolivina robusta Brady - LeRoy (Part 1), p. 33, pl. 1, figs. 75, 76; LeRoy (Part 2), p. 80 , pl. 1, fig. 2, pl. 2, figs. 9, 10.

1958 Bolivina robusta Brady - Asano, pp. 20, 21 , pl. 5, figs. $1-3,5,6$.

1964 Bolivina robusta Brady - LeRoy, p. F31, pl. 2, fig. 13.

1966 Bolivina robusta Brady - Belford, pp. 21, 23, pl. 1, figs. 5-7, text-fig. 4-10.

1968 Bolivina robusta Brady - Huang, p. 56, pl. 13, fig. 14.

1977 Bolivina cf. robusta Brady - McCulloch, p. 257, pl. 105, fig. 17.

1988 Bolivina robusta Brady - Wang, Zhang, Zhao, Min, Bian, Zheng, Cheng, and Chen, p. 147 , pl. 34 , figs. $1-5$; Marle, p. 139, pl. 1, figs. 25, 26.

1989 Bolivina robusta Brady - Ōki, p. 110, pl. 10, fig. 1; Inoue, p. 150, pl. 20, fig. 6, pl. 23, fig. 1, pl. 31, fig. 5 .

1990 Bolivina robusta Brady - Akimoto, pp. 192, 193, pl. 16, fig. 1, pl. 22, fig. 13.

1994 Bolivina robusta Brady - Jones, p. 58, pl. 53, figs. 7-9; Loeblich and Tappan, p. 111 , pl. 215, figs. 17, 18.

1998 Bolivina robusta Brady - Hess, p. 76, pl. 10, fig. 3.

2002 Bolivina robusta Brady - Akimoto, Matsui, Shimokawa, and Furukawa, p. 14, pl. 38, fig. 5 .

Occurrence. Common to abundant in all the formations of the present study.

\section{Bolivina striatula Cushman}

Figures 22.13, 22.14

1922

Bolivina striatula Cushman (a), p. 27, pl. 3, fig. 10.

1942 Bolivina striatula Cushman - Cushman, pp. 30, 31, pl. 9, fig. 1.

1964 Bolivina striatula Cushman - LeRoy, p. F31, pl. 2, fig. 8.

1989 Bolivina striatula Cushman - Ōki, pp. 111, 171, pl. 10, fig. 3.

1992 Bolivina striatula Cushman - Ōki and Yamamoto, pp. 195, 196, fig. 4.9.

1993 Brizalina gr. striatula Cushman - Haig, pl. 6, figs. 7-10. 
1998 Bolivina striatula Cushman - Hess, p. 76, pl. 10, fig. 4.

1999 Bolivina striatula Cushman - Fujita, Nishi, and Saito, pl. 2, fig. 3.

2006 Brizalina striatula (Cushman) - Takata, Irizuki, and Ishida, pl. 1, fig. 4.

Occurrence. Sporadic in the Onogoshi, Yonahama, and Minebari formations.

\section{Bolivina cf. subspinescens Cushman}

Figure 22.23

Compared with:

1922 Bolivina subspinescens Cushman (b), p. 48 , pl. 7 , fig. 5 .

Remarks. Our single specimen is broken and poorly preserved, so that critical identification is difficult.

Occurrence. Yonahama Formation.

\section{Bolivina translucens Phleger and Parker}

Figure 22.27

1951 Bolivina translucens Phleger and Parker, p. 15, pl. 7, figs. 13, 14.

Remarks. Inflated, slightly transparent chambers, and non-limbated sutures distinguish this species from others.

Occurrence. A single specimen from the Minebari Formation.

\section{Bolivina variabilis (Williamson)}

Figures 22.15, 22.16

1858 Textularia variabilis Williamson, p. 76, pl. 6, figs. 162, 163.

1924 Bolivina variabilis (Williamson) - Cushman, pp. 32, 33, pl. 9, fig. 3.

1989 Bolivina variabilis (Williamson) - Ōki, p. 112, pl. 10, fig. 6.

1992 Bolivina subexcavata Cushman and Wickenden - Hatta and Ujiié (b), p. 171, pl. 25, figs. 2, 3 (non Cushman and Wickenden, 1929).

1993 Bolivina sp. - Haig, pl. 6, figs. 1-3.

1994 Bolivina variabilis (Williamson) - Loeblich and Tappan, p. 111, pl. 216, figs. 7-15.

1995 Bolivina variabilis (Williamson) - Ujiié, $p$. 60, pl. 91, fig. 5.

1998 Bolivina variabilis (Williamson) - Hess, pl. 19, fig. 6.

Occurrence. Sporadic in the Yonahama Formation.

\section{Bolivina cf. yabei Takayanagi}

Figure 22.24

Compared with:

1953 Bolivina yabei Takayanagi, p. 31, pl. 4, fig. 9.

Remarks. The single specimen from Miyakojima Island is probably a juvenile of $B$. yabei.

Occurrence. Yonahama Formation.

Bolivina sp. A

Figures 22.25, 22.26

Description. Test very small for the genus, less than $0.2 \mathrm{~mm}$ in length; shape typical of genus; periphery rounded; sutures slightly depressed and strongly curved towards periphery; surface almost smooth with fine perforation; aperture interiomarginal but inside of aperture invisible due to sediment fill.

Remarks. This small species may not be detected in studies using sieves of 120-mesh or larger. We cannot find any species to assign this form to; we refrain from erecting a new species since it does not show enough characters to discriminate it from the vast amount of established bolivinid species.

Occurrence. Sporadic in the Onogoshi, Yonahama, and Minebari formations.

Genus LATIBOLIVINA Srinivasan, 1966 Latibolivina subreticulata (Parr)

Figures 23.1, 23.2

1932 Bolivina subreticulata Parr (a), p. 12, pl. 1, fig. 21.

1942 Bolivina subreticulata Parr - Cushman, pp. 31, 32, pl. 9, fig. 2.

1944 Bolivina subreticulata Parr - LeRoy (Part 1), p. 29, pl. 8, figs. 21, 22.

1964 Bolivina subreticulata Parr — LeRoy, p. F31, pl. 2, fig. 16.

1966 Brizalina subreticulata (Parr) - Belford, pp. 29,30 , pl. 1 , figs. 17,18 , text-figs. $3-$ 1-3-3.

1988 Brizalina subreticulata (Parr) - Wang, Zhang, Zhao, Min, Bian, Zheng, Cheng, and Chen, p. 147, pl. 20, fig. 5.

1988 Bolivina subreticulata Parr - Marle, p. 139, pl. 5, fig. 4.

1989 Bolivina subreticulata Parr - Ōki, p. 111, pl. 10, fig. 4; Inoue, pl. 20, fig. 11.

1992 Brizalina subreticulata (Parr) - Hatta and Ujiié (b), p. 171, pl. 25, fig. 4.

1994 Brizalina subreticulata (Parr) - Jones, p. 59, pl. 53, figs. 30, 31. 
1994 Latibolivina subreticulata (Parr) - Loeblich and Tappan, p. 112, pl. 217, figs. 111.

Remarks. We assign this species to the genus Latibolivina mainly on the basis of its well-developed rib ornament, whereas Revets (1996a) regarded Latibolivina as a junior synonym of Bolivina, and Jones (1994) as Brizalina.

Occurrence. Sporadic in the Minebari Formation. Superfamily BOLIVINITOIDEA Cushman, 1927a

Family BOLIVINITIDAE Cushman, 1927a

Genus ABDITODENTRIX Patterson, 1985

Abditodentrix pseudothalmanii (Boltovskoy and Guissani de Kahn)

Figures 23.3, 23.4

1981 Bolivinita pseudothalmanni Boltovskoy and Guissani de Kahn, pp. 44-46, pl. 1, figs. $1-5$.

1985 Abditodentrix asketocomptella Patterson, p. 140 , pl. 1, figs. $1-9$.

1987 Abditodentrix pseudothalmanni (Boltovskoy and Guissani de Kahn) Loeblich and Tappan, pl. 554, figs. 1-5.

1988 Bolivinita suturornata Zheng, in Wang, Zhang, Zhao, Min, Bian, Zheng, Cheng, and Chen, p. 149, pl. 21, figs. 1, 2.

1990 Abditodentrix pseudothalmanni (Boltovskoy and Guissani de Kahn) Ujié, p. 29, 30, pl. 12, fig. 2.

1994 Abditodentrix pseudothalmanni (Boltovskoy and Guissani de Kahn) - Xu and Ujiié, p. 516, figs. 6-6-6-8; Loeblich and Tappan, pp. 113, 114, pl. 218, figs. 1 , 2.

1995 Abditodentrix pseudothalmanni (Boltovskoy and Guissani de Kahn) Ujiié, p. 60, pl. 4, fig. 7.

2001 Abditodentrix pseudothalmanni (Boltovskoy and Guissani de Kahn) Hayward, Carter, Grenfell, and Hayward, p. 583, figs. 14-J, 14-K.

Occurrence. Sporadic in the Yonahama Formation, a single specimen from the Minebari Formation.

Genus BOLIVINITA Cushman, 1927a

Bolivinita quadrilatera (Schwager)

Figures 23.5, 23.6

1866 Textilaria quadrilatera Schwager, p. 253, pl. 7, fig. 10.

1899 Textularia quadrilatera Schwager - Millett (part VI), p. 559, pl. 7, fig. 3.
1911 Textularia quadrilatera Schwager - Cushman, pp. 24-26, figs. 42-44.

1941 Bolivinita quadrilatera (Schwager) LeRoy (Part 1), p. 31, pl. 1, figs. 99-101; LeRoy (Part 2), p. 79, pl. 2, figs. 3, 4.

1942 Bolivinita quadrilatera (Schwager) Cushman, pp. 2, 3, pl. 1, figs. 1-4.

1944 Bolivinita quadrilatera (Schwager) LeRoy (Part 2), pp. 83, 84, pl. 2, figs. 13, 14.

1958 Bolivinita quadrilatera (Schwager) Asano, pp. 27, 28, pl. 4, fig. 18.

1964 Bolivinita quadrilatera (Schwager) LeRoy, p. F29, pl. 2, figs. 37, 38.

1966 Bolivinita quadrilatera (Schwager) - Belford, p. 18, pl. 4, figs. 4-7, text-fig. 1-4.

1977 Bolivinita quadrilatera (Schwager) McCulloch, p. 254, pl. 105, fig. 27.

1989 Bolivinita quadrilatera (Schwager) Inoue, pp. 149, 150, pl. 28, fig. 8.

1990 Bolivinita quadrilatera (Schwager) Akimoto, p. 193, pl. 16, fig. 3.

1994 Bolivinita quadrilatera (Schwager) Jones, p. 47, pl. 42, figs. 8-12; Loeblich and Tappan, p. 114, pl. 219, figs. 7-12; Akimoto, p. 283, pl. 2, fig. 8.

1996 Bolivinita quadrilatera (Schwager) Revets (a), p. 6, pl. 1, figs. 1-4.

Occurrence. Common to abundant in the Yonahama and Minebari formations.

Superfamily CASSIDULINOIDEA d'Orbigny, 1839a

Family CASSIDULINIDAE d'Orbigny, 1839a

Subfamily CASSIDULININAE d'Orbigny, 1839a

Genus CASSIDULINA d'Orbigny, 1826 Cassidulina carinata Silverstri

Figures 23.7, 23.8

1896 Cassidulina laevigata d'Orbigny var. carinata Silvestri, p. 104, pl. 2, fig. 10.

1966 Cassidulina laevigata d'Orbigny - Belford, pp. 138-140, pl. 24, figs. 1-4, text-figs. 16-1, 16-2. (non Cassidulina laevigata d'Orbigny, 1826).

1983 Cassidulina carinata Silvestri - Nomura (a), pl. 3, fig. 5, pl. 4, fig. 5, pl. 23, figs. 69; Nomura (b), pp. $51-53$, pl. 4, figs. 911.

1988 Cassidulina carinata Silvestri - Wang, Zhang, Zhao, Min, Bian, Zheng, Cheng, and Chen, p. 173, pl. 29, fig. 19; Marle, p. 141 , pl. 2, figs. $4,5$. 


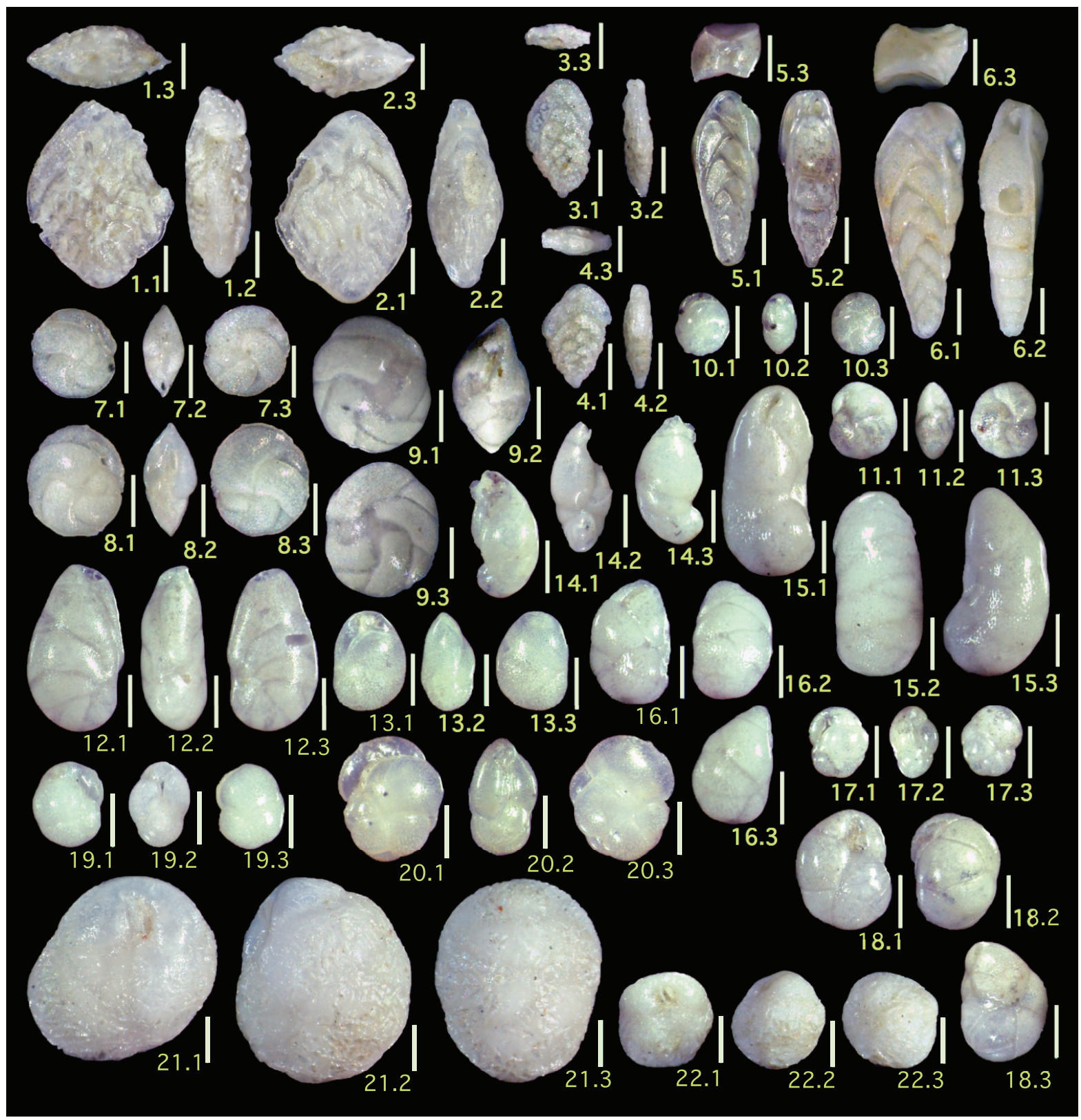

FIGURE 23. Family Bolivinidae, Bolivinitidae, and Cassidulinidae. All scale bars $=0.1 \mathrm{~mm}$. 1, 2. Latibolivina subreticulata (Parr), 1: MPC-26346 from sample 2 (Minebari Formation, PL5); 2: MPC-26347 from sample 5-2 (Minebari F., PL5). 3, 4. Abditodentrix pseudothalmanni (Boltovskoy and Guissani de Kahn), 3: MPC-26105 from sample 7 (Yonahama F., PL4); 4: MPC-26106 from sample 15 (Yonahama F., PL3). 5, 6. Bolivinita quadrilatera (Schwager), 5: MPC26166 from sample 5-2 (Minebari F., PL5); 6: MPC-26167 from sample MK02B (Minebari F., PL5). 7, 8. Cassidulina carinata Silvestri, 7: MPC-26189 from sample MK01 (Yonahama F., PL2); 8: MPC-26190 from sample MK02B (Minebari F., PL5). 9. Cassidulina cf. laevigata d'Orbigny, MPC-26191 from sample 2-2 (Minebari F., PL5). 10, 11. Cassidulina reniforme Nørvang, 10: MPC-26192 from sample 1 (Minebari F., PL5); 11: MPC-26193 from sample 11 (Onogoshi F., ?PL1). 12, 13. Evolvocassidulina belfordi Nomura, 12: MPC-26249 from sample 14-2 (Yonahama F., PL4); 13: MPC-26250 from sample MK02B (Minebari F., PL5). 14. Evolvocassidulina bradyi (Norman), MPC-26251 from sample 1 (Minebari F., PL5). 15, 16. Evolvocassidulina brevis (Aoki), 15: MPC-26252 from sample 5 (Minebari F., PL5); 16: MPC-26253 from sample 5-2 (Minebari F., PL5). 17, 18. Globocassidulina bisecta Nomura, 17: MPC26284 from sample 1 (Minebari F., PL5); 18: MPC-26285 from sample 11 (Onogoshi F., ?PL1). 19, 20. Globocassidulina crassa (d'Orbigny), 19: MPC-26286 from sample MK01 (Yonahama F., PL2); 20: MPC-26287 from sample MK02B (Minebari F., PL5). 21, 22. Globocassidulina decorata (Sidebottom), 21: MPC-26288 from sample 8-2 (Yonahama F., PL4); 22: MPC-26289 from sample 14 (Yonahama F., PL4). 
1990 Cassidulina carinata Silvestri - Akimoto, p. 194 , pl. 17, fig. 3.

1992 Cassidulina carinata Silvestri - Kaiho, pl. 3, fig. 4.

1994 Cassidulina laevigata carinata Silvestri Jones, p. 60, pl. 54, figs. 2, 3 .

1994 Cassidulina carinata Silvestri - Loeblich and Tappan, p. 114, pl. 220, figs. 7-12.

2000 Cassidulina carinata Silvestri - Ohkushi, Thomas, and Kawahata, pl. 2, fig. 1.

2001 Cassidulina carinata Silvestri - Hayward, Carter, Grenfell, and Hayward, fig. 14-FF.

Remarks. Nomura (1983a, b) studied the taxonomy of Cassidulinidae in detail; we basically followed his views here.

Occurrence. Common in all the formations of the present study.

Cassidulina cf. laevigata d'Orbigny

Figure 23.9

Compared with:

1987 Cassidulina laevigata d'Orbigny - Loeblich and Tappan, pl. 555, figs. $1-5$.

Remarks. The single specimen is broken in the apertural part and critical identification is difficult.

Occurrence. Minebari Formation.

Cassidulina reniforme Nørvang

Figures 23.10, 23.11

1945 Cassidulina crassa d'Orbigny var. reniforme Nørvang, p. 41, text-figs. 6e-6h.

1945 Cassidulina islandica Nørvang, forma minuta Nørvang, p. 43, text-fig. 8.

1952 Cassidulina islandica Norvang var. norvangi Thalmann in Phleger, p. 83, footnote 1.

1983 Cassidulina norvangi Thalmann Nomura (a), pl. 23, figs. 10-12, pl. 24, figs. 1-3; Nomura (b), pl. 4, figs. 12, 13.

1989 Islandiella norvangi (Thalmann) - Inoue, pl. 23, fig. 10, fig. 14, pl. 33, fig. 10.

1990 Cassidulina norvangi Thalmann - Ujiié, $p$. 38, pl. 18, figs. 4,5 .

1995 Cassidulina norvangi Thalmann - Ujiié, pp. 60, 61, pl. 4, fig. 10.

1999 Cassidulina reniforme Nørvang Nomura, p. 43, figs. 18-18, 18-19, 30-3.

2000 Cassidulina norvangi Thalmann Ohkushi, Thomas, and Kawahata, p. 139, pl. 2, fig. 6 .
Occurrence. Sporadic in the Onogoshi, Yonahama, and Minebari formations.

Genus EVOLVOCASSIDULINA Eade, 1967 Evolvocassidulina belfordi Nomura

Figures 23.12, 23.13

1964 Cassidulinoides braziliensis (Cushman) LeRoy, p. F41, pl. 12, figs. 3, 4 (non Cassidulina braziliensis Cushman, 1922b).

1983 Evolvocassidulina belfordi Nomura (a), pp. 79-82, pl. 2, fig. 6, pl. 20, figs. 8-10, 12.

Occurrence. Common in the Yonahama and Minebari formations.

Evolvocassidulina bradyi (Norman)

Figure 23.14

1880 Cassidulina bradyi Norman in Wright, p. 152.

1966 Cassidulinoides bradyi (Norman) - Belford, pp. 53, 54, pl. 26, figs. 22-27, textfigs. 17-17, 17-18.

1983 Evolvocassidulina bradyi (Norman) Nomura (b), p. 48, pl. 4, fig. 3.

1990 Evolvocassidulina bradyi (Norman) Ujiié, p. 38, pl. 18, fig. 8.

1994 Cassidulinoides bradyi (Norman) - Jones, p. 60 , pl. 54 , figs. $6-9$.

Occurrence. A single specimen from the Minebari Formation.

\section{Evolvocassidulina brevis (Aoki)}

Figures 23.15, 23.16

1968 "Cassidulina" brevis Aoki, p. 261, pl. 27, fig. 4.

1983 Evolvocassidulina brevis (Aoki) - Nomura (a), pl. 20, fig. 11, pl. 21, figs. 1-5; Nomura (b), pp. 49, 50, pl. 4-7.

1988 Cassidulinoides bradyi (Norman) - Marle, p. 141, pl. 5, fig. 20 (non Cassidulina bradyi Norman in Wright, 1880).

1990 Evolvocassidulina brevis (Aoki) Akimoto, pp. 197, 198, pl. 17, fig. 5.

1994 Evolvocassidulina brevis (Aoki) - Xu and Ujié, p. 518, figs. 9-1, 9-2; Loeblich and Tappan, p. 114, pl. 221, figs. 7-11.

1995 Evolvocassidulina brevis (Aoki) - Ujiié, p. 61, pl. 5, fig. 3.

2000 Evolvocassidulina brevis (Aoki) Ohkushi, Thomas, and Kawahata, p. 144, pl. 2, fig. 7.

Occurrence. Common in the Minebari Formation. 
Genus GLOBOCASSIDULINA Voloshinova, 1960 Globocassidulina bisecta Nomura

Figures 23.17, 23.18

1983 Globocassidulina bisecta Nomura (a), pp. $73-76$, pl. 2, figs. 2 , 3, pl. 14 , figs. $8-12$, pl. 15 , figs. $1-5$.

1988 Globocassidulina subglobosa (Brady) Wang, Zhang, Zhao, Min, Bian, Zheng, Cheng, and Chen, p. 173, pl. 30, figs. 1, 2.

1994 Globocassidulina bisecta Nomura - Loeblich and Tappan, p. 115, pl. 222, figs. 713; Xu and Ujiié, p. 518, figs. 8, 9.

1995 Globocassidulina bisecta Nomura - Ujiié, p. 61 , pl. 5 , fig. 4 .

2001 Globocassidulina bisecta Nomura Kawagata, p. 88, figs. 8-10.

Occurrence. Sporadic in the Onogoshi, Yonahama, and Minebari formations.

Globocassidulina crassa (d'Orbigny)

Figures 23.19, 23.20

1839 Cassidulina crassa d'Orbigny (c), p. 56, pl. 7, figs. 18-20.

1966 Globocassidulina crassa (d'Orbigny) Belford, pp. 151, 152, pl. 26, figs. 5-9, text-figs. 17-9, 17-10.

1983 Globocassidulina crassa (d'Orbigny) Nomura (a), pl. 18, figs. 3-5; Nomura (b), pp. 37-40, pl. 3, figs. 9, 10, pl. 6, fig. 17.

1994 Cassidulina crassa d'Orbigny - Jones, p. 60 , pl. 54, fig. 4.

2001 Globocassidulina crassa (d'Orbigny) Hayward, Carter, Grenfell, and Hayward, figs. 14-MM, 14-NN.

Occurrence. Sporadic in the Yonahama and Minebari formations.

Globocassidulina decorata (Sidebottom)

Figures 23.21, 23.22

1910 Cassidulina decorata Sidebottom, p. 107, pl. 4, fig. 2.

1983 Globocassidulina decorata (Sidebottom) - Nomura (a), pl. 17, fig. 8; Nomura (b), pp. 27,28 , pl. 2, figs. $14-16$.

1994 Globocassidulina decorata (Sidebottom) - Loeblich and Tappan, p. 115, pl. 222, figs. 14-19.

1995 Globocassidulina decorata (Sidebottom) - Hasegawa and Nomura, pp. 98, 100, fig. 4-3.

Remarks. This species is distinguished from congeners in its reticulate ornament on the test sur- face, but this is occasionally difficult to discern in abraded or partially dissolved specimens.

Occurrence. Sporadic in the Yonahama Formation.

\section{Globocassidulina okinawaensis (LeRoy)}

Figures 24.1, 24.2

1964 Cassidulina okinawaensis LeRoy, p. F40, pl. 11, figs. 21, 22.

1983 Globocassidulina okinawaensis (LeRoy) - Nomura (a), pl. 18, fig. 2; Nomura (b), pp. 29, 30, pl. 2, fig. 20.

Occurrence. Rare in the Minebari Formation, sporadic in the Yonahama Formation.

\section{Globocassidulina subglobosa (Brady)}

Figures 24.3, 24.4

1881 Cassidulina subglobosa Brady, p. 60.

1884 Cassidulina subglobosa Brady - Brady, p. 430, pl. 54, fig. 17.

1911 Cassidulina subglobosa Brady - Cushman, pp. 98, 99, fig. 152.

1965 Cassidulina subglobosa Brady - Todd, p. 45, pl. 16, fig. 7.

1966 Globocassidulina subglobosa (Brady) Belford, p. 149, pl. 25, figs. $11-16$, textfigs. 17-1-17-6, 18-1-18-4.

1968 Cassidulina subglobosa Brady - Huang, p. 56, pl. 13, figs. 15, 27.

1983 Globocassidulina subglobosa (Brady) Nomura (a), pl. 13, figs. 5, 6; Nomura (b), pp. 20—22, pl. 2, figs. 8, 9 .

1988 Globocassidulina subglobosa (Brady) Marle, p. 143, pl. 5, figs. 22.

1990 Globocassidulina subglobosa (Brady) Ujiié, pp. 39, 40, pl. 21, figs. 4-7, pl. 22, fig. 1.

1992 Globocassidulina subglobosa (Brady) Kaiho, pl. 3, figs. 11-15; Kaiho and Nishimura, pl. 3, fig. 23.

1994 Globocassidulina subglobosa (Brady) Jones, p. 60, pl. 54, fig. 17.

1995 Globocassidulina subglobosa (Brady) Ujiié, p. 62, pl. 5, fig. 7.

2000 Globocassidulina subglobosa (Brady) Ohkushi, Thomas, and Kawahata, p. 144, pl. 2, fig. 8.

2001 Globocassidulina subglobosa (Brady) Hayward, Carter, Grenfell, and Hayward, fig. 14-QQ.

Occurrence. Common in the Onogoshi, Yonahama, and Minebari formations. 
Genus LERNELLA Saidova, 1975

Lernella inflata (LeRoy)

Figure 24.5

1944 Cassidulina inflata LeRoy, p. 37, pl. 4, figs. 30, 31.

1983 Lernella inflata (LeRoy) - Nomura (a), pp. 86-88, pl. 2, fig. 9, pl. 24, figs. 4, 5.

1987 Lernella inflata (LeRoy) - Loeblich and Tappan, pl. 559, fig. 10.

1989 Lernella inflata (LeRoy) - Ōki, pl. 19, fig. 2.

1994 Lernella inflata (LeRoy) - Loeblich and Tappan, p. 116, pl. 226, figs. 1-12.

Occurrence. A single specimen from the Minebari Formation.

Genus PARACASSIDULINA Nomura, 1983a

Paracassidulina sagamiensis (Asano and Nakamura)

Figure 24.6

1937 Cassidulina sagamiensis Asano and Nakamura, p. 147, pl. 14, fig. 14.

1983 Paracassidulina sagamiensis (Asano and Nakamura) - Nomura (a), pl. 5, fig. 6, pl. 24 , figs. $10-12$, pl. 25, figs. 1, 2; Nomura (b), pp. 67-69, pl. 6, figs. 1-3.

1995 Paracassidulina sagamiensis (Asano and Nakamura) - Hasegawa and Nomura, p. 98, fig. 3-5.

Occurrence. A single specimen from the Yonahama Formation.

Subfamily EHRENGERGININAE Cushman 1927a Genus BURSEOLINA Seguenza, 1880

Burseolina pacifica (Cushman)

Figures 24.7, 24.8

1925 Cassidulina pacifica Cushman, p. 53, pl. 9, figs. $14-16$.

1941 Cassidulina pacifica Cushman - LeRoy (Part 1), p. 47, pl. 1, figs. 43-45.

1964 Cassidulina pacifica Cushman - LeRoy, p. F40, 41, pl. 11, figs. 19, 20.

1983 Burseolina pacifica (Cushman) - Nomura (a), pl. 6, fig. 2, pl. 21, figs. 6-10; Nomura (b), pp. 57-60, pl. 5, figs. $1-4$.

1990 Burseolina pacifica (Cushman) - Ujiié, p. 40, pl. 22, fig. 2.

1994 Globocassidulina pacifica (Cushman) Jones, p. 111, pl. 113, fig. 8.

1995 Burseolina pacifica (Cushman) - Hasegawa and Nomura, p. 102, fig. 4-5.
Occurrence. Common in the Minebari Formation.

Genus EHRENBERGINA Reuss, 1850 Ehrenbergina carinata Eade

Figures 24.9, 24.10

1967 Ehrenbergina carinata Eade, p. 448, 450, fig. 8.

1983 Ehrenbergina carinata Eade - Nomura (a), pl. 22, figs. 10-12, pl. 23, figs. 1-4; Nomura (b), pp. 60, 61, pl. 5, figs. 5, 6.

1990 Ehrenbergina carinata Eade - Ujiié, p. 40, pl. 20, figs. 2, 3.

1994 Ehrenbergina carinata Eade - Loeblich and Tappan, p. 117, pl. 228, figs. 1-10.

Occurrence. Rare in the Yonahama Formation.

\section{Ehrenbergina trigona Goës}

Figures 24.11, 24.12

1896 Ehrenbergina serrata Reuss var. trigona Goës, p. 49.

1965 Ehrenbergina trigona (Goës) - Todd, p. 48, pl. 20, fig. 2.

1989 Ehrenbergina trigona (Goës) - Hermelin, p. 73, pl. 13, fig. 13.

1994 Ehrenbergina trigona (Goes) - Jones, p. 61 , pl. 55 , figs. $2-3,5$.

Occurrence. Sporadic in the Minebari Formation.

Superfamily TURRILINOIDEA Cushman, 1927a

Family STAINFORTHIIDAE Reiss, 1963

Genus STAINFORTHIA Hofker, 1956a

Stainforthia ishikiensis (Asano)

Figures 24.13, 24.14

1949 Virgulina ishikiensis Asano, p. 428, text-fig. 1.

Remarks. This species was originally described from the Miocene of Fukushima, northern Japan. Scott et al. (2000) suggested it was close to Fursenkoina fusiformis [= Bulimina pupoides d'Orbigny var. fusiformis Williamson, 1858, p. 64, pl. 5, figs. 129, 130] as well as to Virgulina akitaensis Iwasa (1955, p. 17, text-fig. 2); but both species are distinguished from $F$. fusiformis in their more slender shape.

Occurrence. Rare in the Yonahama and Minebari formations.

\section{Stainforthia tenuata (Cushman)}

Figures 25.1, 25.2

1927 Buliminella subfusiformis Cushman var. tenuata Cushman (b), p. 149, pl. 2, fig. 9.

1958 Bulimina exilis tenuata (Cushman) Asano, p. 4, pl. 3, figs. 11, 12. 


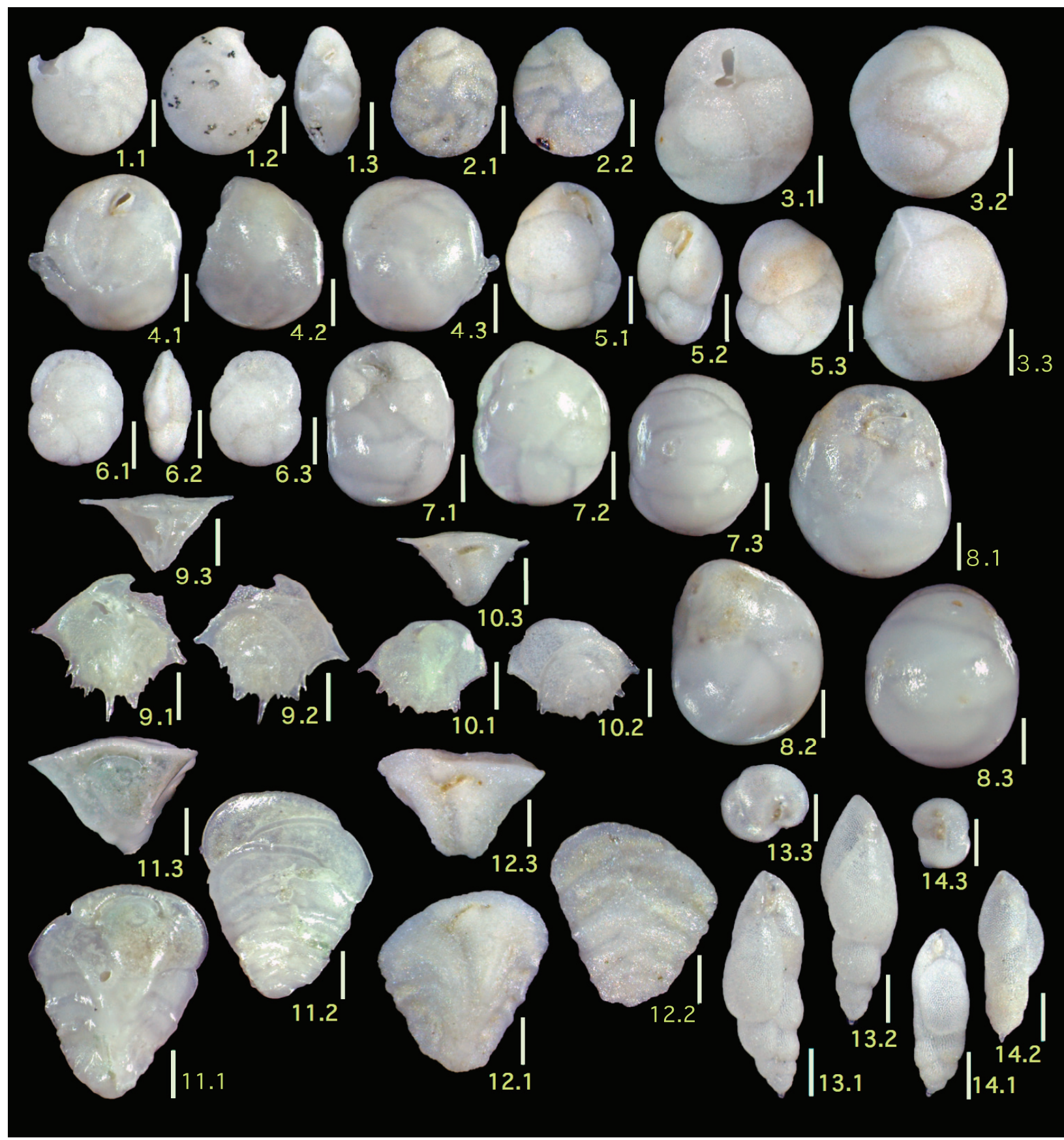

FIGURE 24. Family Cassidulinidae and Stainforthiidae. All scale bars $=0.1 \mathrm{~mm}$. 1, 2. Globocassidulina okinawaensis (LeRoy), 1: MPC-26290 from sample 14-2 (Yonahama Formation., PL4); 2: MPC-26291 from sample MK02A (Minebari F., PL5). 3, 4. Globocassidulina subglobosa (Brady), 3: MPC-26292 from sample 17 (Yonahama F., PL2); 4: MPC26293 from sample MK01 (Yonahama F., PL2). 5. Lernella inflata (LeRoy), MPC-26366 from sample 12 (Minebari F., PL5). 6. Paracassidulina sagamiensis (Asano and Nakamura), MPC-26403 from sample 7 (Yonahama F., PL4). 7, 8. Burseolina pacifica (Cushman), 7: MPC-26185 from sample 2 (Minebari F., PL5); 8: MPC-26186 from sample 2-2 (Minebari F., PL5). 9, 10. Ehrenbergina carinata Eade, 9: MPC-26226 from sample 14 (Yonahama F., PL4); 10: MPC26227 from sample 14-2 (Yonahama F., PL4). 11, 12. Ehrenbergina trigona Goës, 11: MPC-26228 from sample 2 (Minebari F., PL5); 12: MPC-26229 from sample MK02A (Minebari F., PL5). 13, 14. Stainforthia ishikiensis (Asano), 13: MPC-26508 from sample 14 (Yonahama F., PL4); 14: MPC-26509 from sample 14-2 (Yonahama F., PL4). 
1989 Stainforthia exilis tenuata (Cushman) Inoue, pp. 151, 152, pl. 32, fig. 3, pl. 33, fig. 7.

1990 Stainforthia exilis tenuata (Cushman) Ujiié, p. 30, pl. 12, fig. 7.

Occurrence. Rare in the Onogoshi and Yonahama formations.

Superfamily BULIMINOIDEA Jones in Griffith and Henfrey, 1875

Family SIPHOGENERINOIDIDAE Saidova, 1981

Subfamily SIPHOGENERINOIDINAE Saidova, 1981

Genus EULOXOSTOMUM McCulloch, 1977

Euloxostomum bradyi (Asano)

Figures 25.3, 25.4

1938 Bolivina bradyi Asano (c), p. 603, pl. 16, fig. 2.

1958 Loxostomum bradyi (Asano) - Asano, p. 25, pl. 5, fig. 7.

1987 Euloxostomum bradyi (Asano) - Loeblich and Tappan, pl. 566, figs. 19-23.

1994 Euloxostomum bradyi (Asano) - Jones, p. 58 , pl. 53, fig. 1.

Occurrence. Rare in the Yonahama and Minebari formations.

Euloxostomum pseudobeyrichi (Cushman)

Figures 25.5, 25.6

1911 Bolivina beyrichi Reuss, var. alata (Seguenza) - Cushman, p. 35, fig. 57 (non Vulvulina alata Seguenza, 1862).

1926 Bolivina pseudobeyrichi Cushman (a), p. 45.

1953 Bolivina pseudobeyrichi Cushman Drooger, p. 131, pl. 21, figs. 9, 10.

1958 Bolivina cf. barbata Phleger and Parker Asano, p. 18, pl. 4, fig. 17.

1959 Bolivina pseudobeyrichi Cushman - Blow, p. 147, pl. 6, fig. 22.

1964 Bolivina alata (Seguenza) - LeRoy, p. F31, pl. 2, fig. 12.

1966 Brizarina pseudobeyrichi (Cushman) Belford, pp. 28, 29, pl. 1, figs. 13-16, textfigs. 2-4-2-6.

1988 Brizalina alata (Seguenza) - Wang, Zhang, Zhao, Min, Bian, Zheng, Cheng, and Chen, p. 148, pl. 20, fig. 18.

1988 Brizalina pseudobeyrichi (Cushman) Marle, p. 139, pl. 5, figs. 5, 6.
1989 Bolivina alata (Seguenza) - Inoue, pl. 33, fig. 5.

1994 Brizalina alata (Seguenza) - Jones, p. 58, pl. 53, figs. 2-4.

1994 Euloxostomum pseudobeyrichi (Cushman) - Loeblich and Tappan, p. 118, pl. 231, figs. 9-23.

Remarks. The relationship between $B$. alata and $B$. pseudobeyrichi was discussed by Drooger (1953), Blow (1959), Belford (1966), and others, and we conclude they are distinct species. This species also resembles Bolivina barbata Phleger and Parker (1951), but is differentiated by its fewer chambers and less rapid growth rate as pointed out by Asano (1958).

Occurrence. Rare in the Onogoshi, Yonahama, and Minebari formations.

Genus HOPKINSINELLA Bermúdez and Fuenmayor, 1966 Hopkinsinella glabra (Millett)

Figures 25.7, 25.8

1903 Uvigerina auberiana d'Orbigny var. glabra Millett (part XIV), p. 268, pl. 5, figs. 8, 9.

1933 Hopkinsina pacifica Cushman (b), p. 86, pl. 8, fig. 16.

1942 Hopkinsina pacifica Cushman - Cushman, p. 51, pl. 15, fig. 1.

1977 Hopkinsina cf. pacifica Cushman McCulloch, p. 263, pl. 102, figs. 21, 22.

1988 Hopkinsina pacifica Cushman - Wang, Zhang, Zhao, Min, Bian, Zheng, Cheng, and Chen, p. 155, pl. 30, fig. 3.

1989 Hopkinsina glabra (Millett) - Ōki, p. 120, pl. 12, fig. 6.

1992 Hopkinsina glabra (Millett) - Ōki and Yamamoto, p. 197, fig. 5-3.

1993 Hopkinsinella glabra (Millett) - Haig, pl. 1, figs. 15-17.

1994 Hopkinsinella glabra (Millett) - Loeblich and Tappan, pp. 118, 119, pl. 232, figs. 111.

1996 Hopkinsinella glabra (Millett) - Revets (a), p. 14 , pl. 10, figs. $5-8$.

2002 Hopkinsina pacifica Cushman - Akimoto, Matsui, Shimokawa, and Furukawa, p. 15, pl. 43, fig. 1.

Remarks. Hopkinsinella pacifica is regarded as a junior synonym of the present species.

Occurrence. Sporadic in the Yonahama and Minebari formations. 


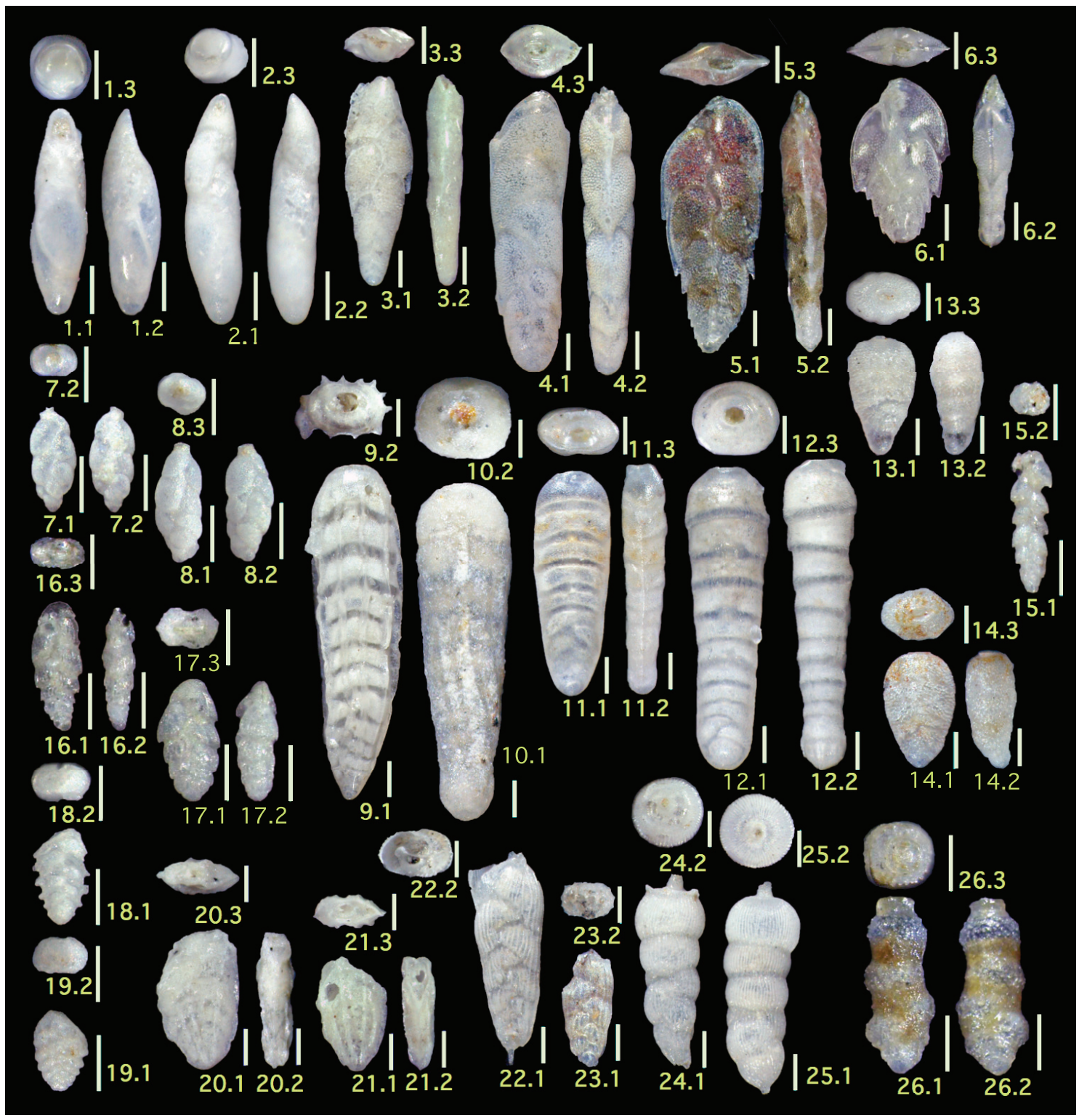

FIGURE 25. Family Stainforthiidae and Siphogenerinoididae. All scale bars $=0.1 \mathrm{~mm}$. 1, 2. Stainforthia tenuata (Cushman), 1: MPC-26510 from sample 8-2 (Yonahama Formation, PL4); 2: MPC-26511 from sample 15 (Yonahama F., PL3). 3, 4. Euloxostomum bradyi (Asano), 3: MPC-26239 from sample 8-2 (Yonahama F., PL4); 4: MPC-26240 from sample MK02B (Minebari F., PL5). 5, 6. Euloxostomum pseudobeyrichi (Cushman), 5: MPC-26241 from sample 5 (Minebari F., PL5); 6: MPC-26242 from sample 5-2 (Minebari F., PL5). 7, 8. Hopkinsinella glabra (Millett), 7: MPC26326 from sample 1 (Minebari F., PL5); 8: MPC-26327 from sample 14-2 (Yonahama F., PL4). 9, 10. Rectobolivina asanoi Murata, 9: MPC-26453 from sample 18 (Oura F., ?PL1); 10: MPC-26454 from sample MK02A (Minebari F., PL5); 9 microspheric form, 10 megalospheric form. 11, 12. Rectobolivina bifrons (Brady), 11: MPC-26455 from sample 17 (Yonahama F., PL2); 12: MPC-26456 from sample MK01 (Yonahama F., PL2); 11 microspheric form, 12 megalospheric form. 13, 14. Rectobolivina sp.A, 13: MPC-26457 from sample 6 (Yonahama F., PL2); 14: MPC-26458 from sample 17 (Yonahama F., PL2). 15. Sagrinella convallaria (Millett), MPC-26476 from sample 10 (Onogoshi F., PL2). 16, 17. Sagrinella durrandii (Millett), 16: MPC-26477 from sample 1 (Minebari F., PL5); 17: MPC-26478 from sample 5 (Minebari F., PL5). 18, 19. Sagrinella jugosa (Brady), 18: MPC-26479, 19: MPC-26480, both from sample MK01 (Yonahama F., PL2). 20, 21. Saidovina formosana (Nakamura), 20: MPC-26481, 21: MPC-26482, both from sample 18 (Oura F., ?PL1). 22, 23. Saidovina karreriana (Brady), 22: MPC-26483 from sample 5 (Minebari F., PL5); 23: MPC-26484 from sample MK02B (Minebari F., PL5). 24, 25. Rectuvigerina striata (Schwager), 24: MPC-26459 from sample 8-2 (Yonahama F., PL4); 25: MPC-26460 from sample 14 (Yonahama F., PL4). 26. Allassoida tonohamaensis (Takayanagi), MPC-26110 from sample 1-2 (Minebari F., PL5). 
Genus RECTOBOLIVINA Cushman, 1927a Rectobolivina asanoi Murata

Figures 25.9, 25.10

1951 Rectobolivina asanoi Murata, p. 96, pl. 1, text-fig. 2.

1952 Rectobolivina asanoi Murata - Asano (supplement 1), p. 13, figs. 70, 71.

2001 Rectobolivina asanoi Murata - Kawagata, pp. 88, 89, figs. 8-13.

2002 Rectobolivina asanoi Murata - Kawagata and Hatta, p. 223, fig. 4.1, 4.2, 10.1, 10.2, 13.1.

Remarks. This species was originally described from the upper Miocene of Kyushu, southwest Japan. Kawagata and Hatta (2002) thoroughly described the taxonomy of the genus Rectobilivina; we here follow their views.

Occurrence. Abundant in the Oura Formation, common in the Onogoshi and Minebari formations.

\section{Rectobolivina bifrons (Brady)}

Figures 25.11, 25.12

1881 Sagrina bifrons Brady, p. 64

1913 Siphogenerina bifrons (Brady) - Cushman, pp. 105, 106, pl. 45, figs. 1, 2, 5-7.

1958 Rectobolivina bifrons (Brady) - Asano, pp. 28, 29, pl. 5, figs. 10, 11 .

1964 Rectobolivina bifrons (Brady) - LeRoy, $p$. F34, pl. 3, figs. 1, 2.

1966 Rectobolivina bifrons (Brady) - Belford, pp. 45, 46, pl. 9, figs. 13, 14.

1989 Rectobolivina bifrons (Brady) - Inoue, pl. 28, fig. 6.

1994 Rectobolivina bifrons (Brady) - Jones, p. 87, pl. 75, figs. 18-20; Loeblich and Tappan, p. 120, pl. 234, figs. 13, 14.

2002 Rectobolivina bifrons (Brady) - Kawagata and Hatta, pp. 223, 226, figs. 4.3, 4.4, 8.2-8.5, 13.2-13.4.

Occurrence. Rare in the Onogoshi and Yonahama formations, common in the Minebari Formation.

Rectobolivina sp. A

Figures 25.13, 25.14

1961 ?Siphogenerina aff. dimorpha (Parker and Jones) var. pacifica Cushman - LeRoy (Part 1), p. 37, pl. 3, figs. 97, 98.

Description. Test small and short for the genus, approximately $0.4 \mathrm{~mm}$ in length, $0.2 \mathrm{~mm}$ in breadth, $0.1 \mathrm{~mm}$ in thickness; triangular in front view, oval in transverse section; chambers not inflated, increasing slowly as added; surface wall coarsely pitted with pores; sutures slightly depressed, weakly limbate; aperture oval with marginal rim at the top of test.

Remarks. This species resembles Siphogenerina aff. dimorpha var. pacifica Cushman as illustrated by LeRoy (1961), in having a compressed test with coarsely pored wall. Siphogenerina dimorpha var. pacifica Cushman (1926) has a cylindrical test with a near-circular cross section.

Occurrence. Common in the lower part of the Yonahama Formation.

\section{Genus SAGRINELLA Saidova, 1975} Sagrinella convallaria (Millett)

Figure 25.15

1900 Bolivina convallaria Millett (part IX), p. 544, pl. 4, fig. 6.

1942 Loxostoma convallarium (Millett) - Cushman, p. 37, pl. 10, fig. 6 .

1959 Loxostomum convallarium (Millett) - Graham and Militante, p. 83, pl. 12, fig. 27.

1964 Bitubulogenerina convallaria (Millett) LeRoy, p. F34, pl. 2, fig. 28.

1993 "Sagrina" gr. convallarium (Millett) - Haig, pl. 2, figs. 15-17.

1994 Sagrinella convallaria (Millett) - Loeblich and Tappan, p. 120, pl. 236, figs. 1-8.

2002 Sagrinella convallaria (Millett) - Akimoto, Matsui, Shimokawa, and Furukawa, p. 15, pl. 40 , fig. 3.

Occurrence. A single specimen from the Onogoshi Formation.

Sagrinella durrandii (Millett)

Figures 25.16, 25.17

1900 Bolivina durrandii Millett (part IX), p. 544, pl. 4, fig. 7.

1989 Bolivina durrandii Millett — Ōki, p. 107, pl. 8, fig. 14.

1992 Loxostoma durrandii (Millett) - Hatta and Ujiié (b), p. 174, pl. 26, fig. 10.

1992 Bolivina durrandii Millett — Ōki and Yamamoto, p. 195, fig. 4-7.

1994 Sagrinella scutata Saidova - Loeblich and Tappan, p. 121, pl. 236, figs. 9, 10.

Non 1994Sagrinella durrandii Saidova - Loeblich and Tappan, p. 120, pl. 236, figs. 11-13.

Remarks. Sagrinella durrandii of Loeblich and Tappan (1994) has a compressed test and differs from the present species.

Occurrence. Rare in the Yonahama and Minebari formations. 
Sagrinella jugosa (Brady)

Figures 25.18, 25.19

1884 Textularia jugosa Brady, p. 358, pl. 42, fig. 7.

1899 Textularia inconspicua var. jugosa Brady — Millett (part VI), p. 558, pl. 7, fig. 2.

1964 Patellinella jugosa (Brady) - LeRoy, pp. F35, F36, pl. 2, figs. 33, 34.

1988 Patellinella jugosa (Brady) - Marle, p. 148, pl. 4, figs. 14, 15.

1993 Sagrinella jugosa (Brady) - Haig, pl. 2, figs. 21-23.

1994 Sagrinella jugosa (Brady) - Jones, p. 47, pl. 42, fig. 7.

1994 Sagrina jugosa (Brady) - Loeblich and Tappan, p. 122, pl. 237, figs. 12-17.

1996 Sagrinella jugosa (Brady) - Revets (a), p. 11, pl. 6, figs. 5-8.

Remarks. Geminaricta pacifica Asano (1938c, p. 608 , pl. 16, fig. 16 ; 1958, p. 27, pl. 5, figs. 16, 17) and Suggrunda yahikoensis Matsunaga (1963, p. 111, pl. 41, figs. 10, 11) resemble this species, but these two have a less developed ridge on sutures than does $S$. jugosa.

Occurrence. Nine specimens from a single sample of the Yonahama Formation.

Genus SAIDOVINA Haman, 1984

Saidovina formosana (Nakamura)

Figures 25.20, 25.21

1937 Bolivina formosana Nakamura, p. 140, pl. 12, fig. 2.

Occurrence. Two specimens from the Oura Formation.

Saidovina karreriana (Brady)

Figures 25.22, 25.23

1881 Bolivina karreriana Brady, p. 28.

1911 Bolivina karreriana Brady - Cushman, p. 40, fig. 66.

1921 Bolivina karreriana Brady - Cushman, $p$. 131, pl. 26, fig. 4.

1938 Loxostoma karrerianum Brady - Asano (c), p. 605 , pl. 16, fig. 6 .

1958 Loxostomum karrerianum (Brady) Asano, pp. 25, 26, pl. 5, figs. 8, 9.

1964 Loxostomum karrerianum (Brady) LeRoy, p. F33, pl. 2, figs. 26, 27.

1966 Brizalina karreriana (Brady) - Belford, p. 39, pl. 2, figs. 23-25.
1987 Saidovina karreriana (Brady) - Loeblich and Tappan, pl. 568, figs. 7-10.

1989 Loxostomum karrerianum (Brady) Inoue, pl. 31, fig. 10 (non pl. 28, fig. 3).

1990 Brizalina karreriana (Brady) - Akimoto, pl. 16, fig. 2.

1994 Saidovina karreriana (Brady) - Jones, p. 59, pl. 53, figs. 19-21.

1994 Saidovina karreriana (Brady) - Loeblich and Tappan, p. 121, pl. 236, figs. 16-20.

1994 Brizalina karreriana (Brady) - Akimoto, p. 283, pl. 2, fig. 5.

1996 Saidovina karreriana (Brady) - Revets (a), p. 11, pl. 7, figs. 1-4.

1998 Loxostomum karreriana (Brady) - Hess, pl. 10, fig. 5.

Occurrence. Common in the Minebari Formation.

Subfamily TUBULOGENERININAE Saidova, 1981

Genus RECTUVIGERINA Mathews, 1945

Rectuvigerina striata (Schwager)

Figures 25.24, 25.25

1866 Dimorpha striata Schwager, p. 251, pl. 7, fig. 99.

1941 Siphogenerina striata (Schwager) LeRoy, p. 37, pl. 3, figs. 88, 89.

1964 Rectuvigerina striata (Schwager) LeRoy, p. F34, pl. 3, fig. 8.

1966 Rectuvigerina striata (Schwager) - Belford, p. 84, pl. 9, figs. 1, 2.

1968 Siphogenerina multicostata Cushman and Jarvis - Huang, p. 59, pl. 13, fig. 13 (non Siphogenerina multicostata Cushman and Jarvis, 1929).

2002 Rectuvigerina striata (Schwager) - Hayward, pp. 300, 301, pl. 2, figs. 12, 13.

Remarks. Siphogenerina multicostata has more highly raised costae than $R$. striata and is a distinct species.

Occurrence. Sporadic in the Yonahama Formation.

Genus ALLASSOIDA Loeblich and Tappan, 1994 Allassoida tonohamaensis (Takayanagi)

Figure 25.26

1953 Bifarina tonohamaensis Takayanagi, p. 32, pl. 4 , fig. 8.

Remarks. This species has a longer biserial stage and a more compact uniserial stage than Sagrina virgula Brady. Genus Bifarina originally assigned to this species is a Cretaceous/Paleogene plankk- 
tonic taxon. Widely opened round aperture with conspicuous rip characterizes Allassoida.

Occurrence. A single specimen from the Minebari Formation.

Family BULIMINIDAE Jones in Griffith and Henfrey, 1875

Genus BULIMINA d'Orbigny, 1826 Bulimina aculeata d'Orbigny

Figures 26.1, 26.2

1826 Bulimina aculeata d'Orbigny, p. 269.

1911 Bulimina aculeata d'Orbigny - Cushman, pp. 86, 87, fig. 139.

1958 Bulimina aculeata d'Orbigny - Asano, pp. 2, 3, pl. 1, figs. 1-3.

1964 Bulimina aculeata d'Orbigny - LeRoy, p. F30, pl. 11, fig. 7 .

1966 Bulimina aculeata d'Orbigny - Belford, pp. 58,59 , pl. 5 , figs. $1-3$, text-figs. 5-15-3, 7-1.

1988 Bulimina aculeata d'Orbigny - Wang, Zhang, Zhao, Min, Bian, Zheng, Cheng, and Chen, p. 151, pl. 21, fig. 5; Marle, p. 139, pl. 5, fig. 17.

1989 Bulimina aculeata d'Orbigny - Inoue, pp. 150 , 151 , pl. 22, fig. 5 , pl. 28 , fig. 2, pl. 32 , fig. 2.

1990 Bulimina aculeata d'Orbigny - Akimoto, $p$. 193, pl. 16, fig. 5, pl. 22, fig. 9; Ujiié, p. 30, pl. 12, figs. 5, 6 .

1994 Bulimina aculeata d'Orbigny - Xu and Ujié, p. 516, fig. 6-9; Jones, p. 56, pl. 51, figs. 7, 8 (non fig. 9); Akimoto, p. 283, pl. 2, fig. 9.

1994 Bulimina acaenapeza Loeblich and Tappan, p. 123, pl. 243, figs. 1-6.

1998 Bulimina aculeata d'Orbigny - Hess, p. 76, pl. 10, fig. 9.

2000 Bulimina aculeata d'Orbigny - Ohkushi, Thomas, and Kawahata, p. 139, pl. 2, fig. 2.

Remarks. This form is occasionally confused with Bulimina marginata d'Orbigny that has short spines on the acute peripheral margins, whereas $B$. aculeata has a lower spiral with thick and long spines in its bluntly rounded periphery. Loeblich and Tappan (1994) described $B$. acaenapeza as a new species, listing $B$. aculeata of Akimoto (1990) and Ujiié (1990) as synonyms. Despite this, we think $B$. acaenapeza is identical to $B$. aculeata.

Occurrence. Sporadic in the Yonahama Formation, rare in the Minebari Formation.
Bulimina gutta Chapman and Parr Figures 26.5, 26.6

1937 Bulimina buchiana d'Orbigny, var. gutta Chapman and Parr, p. 86, pl. 8, fig. 14.

1947 Bulimina buchiana d'Orbigny, var. gutta Chapman and Parr - Cushman and Parker, p. 127, pl. 29, fig. 16.

1966 Laterostomella voluta Belford, pp. 44, 45, pl. 3, figs. 14-16.

1977 Bulimina cf. buchiana gutta Chapman and Parr - McDougall, pp. 244, 245, pl. 104, figs. 10, 11.

1989 Laterostomella striata Klasz and Rérat Klasz, Kroon, and Hinte, pp. 215-226.

Remarks. This species is characterized by an elongate and slender test with raised striae that persist to the penultimate chamber. Chamber arrangement of the earlier stage is difficult to observe due to the raised striae; later chambers are strongly twisted which suggests a triserial early stage. The genus Laterostomella sensu Klasz et al. (1989) is biserial; species belonging to the genus show a clearly different form from the present species.

Occurrence. Common in the Yonahama Formation.

\section{Bulimina inflata Sequenza}

Figures 26.7, 26.8

1862 Bulimina inflata Seguenza, p. 25, pl. 1, fig. 10.

1941 Bulimina inflata Seguenza - LeRoy (Part 1), p. 32, pl. 2, figs. 71, 72; LeRoy (Part 2), p. 79 , pl. 1, fig. 5 .

1944 Bulimina inflata Seguenza - LeRoy (Part 2), p. 85, pl. 7, fig. 21.

1964 Bulimina inflata Seguenza - LeRoy, p. F30, pl. 11, fig. 6 .

1966 Bulimina striata d'Orbigny - Belford, pp. 59,61 , pl. 5 , figs. $6-8$, text-figs. $5-7,5-8$, 7-11.

Remarks. This species resembles $B$. striata, but can be differentiated by its robust and inflated chambers and raised costae covering almost all surface of chambers close to the shoulder of the ultimate chamber.

Occurrence. Abundant in only a single sample of the Yonahama Formation.

\section{Bulimina aff. kochiensis Takayanagi}

Figures 26.3, 26.4

Compared with: 


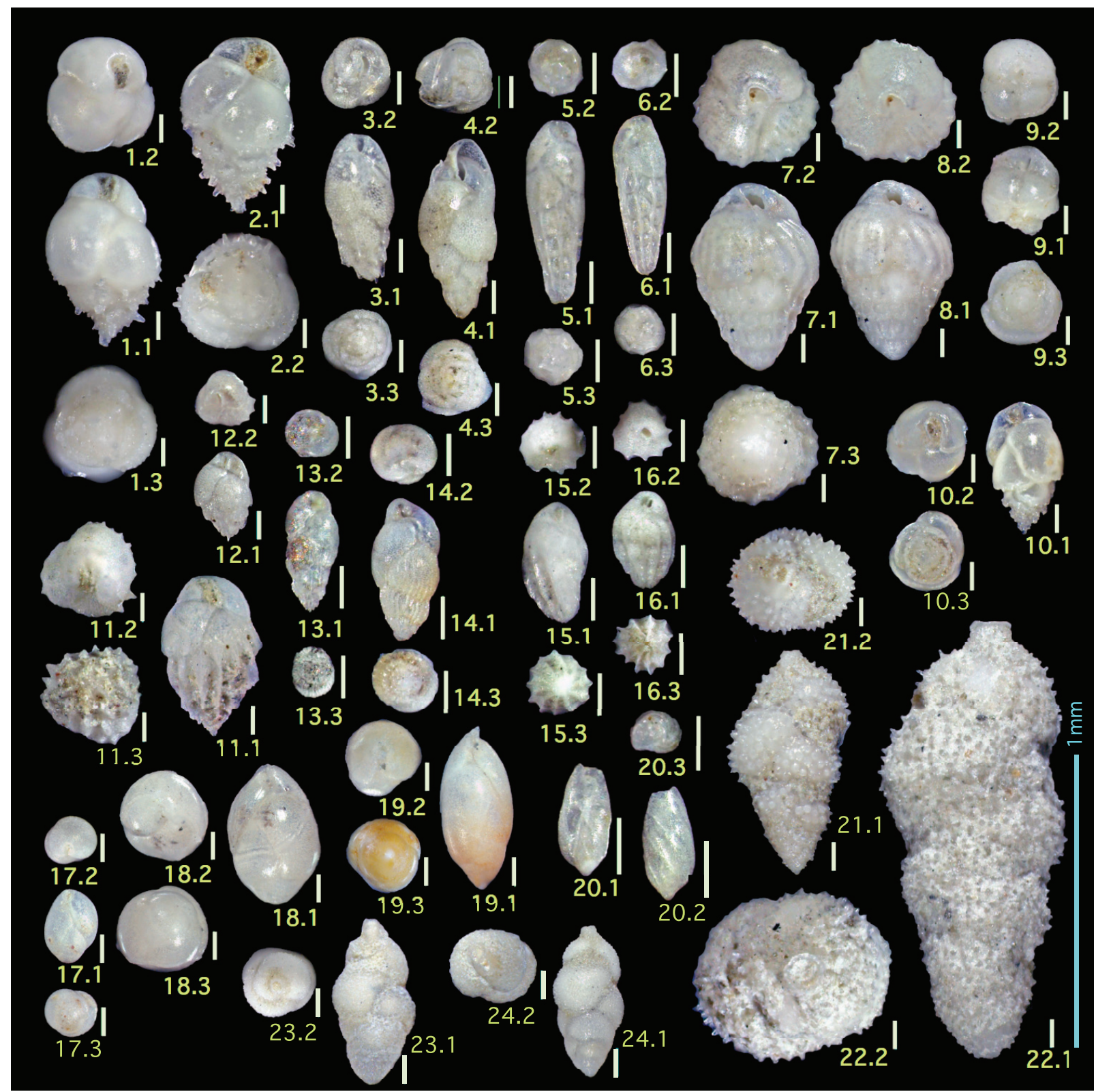

FIGURE 26. Family Buliminidae, Buliminellidae, and Uvigerinidae. All scale bars $=0.1 \mathrm{~mm}$, unless otherwise indicated. 1, 2. Bulimina aculeata d'Orbigny, 1: MPC-26168 from sample 8 (Yonahama Formation, PL4); 2: MPC-26169 from sample 8-2 (Yonahama F., PL4). 3, 4. Bulimina aff. kochiensis Takayanagi, 3: MPC-26174 from sample 1 (Minebari F., PL5); 4: MPC-26175 from sample 2 (Minebari F., PL5). 5, 6. Bulimina gutta Cushman and Parr, 5: MPC26170 from sample 14 (Yonahama F., PL4); 6: MPC-26171 from sample 14-2 (Yonahama F., PL4). 7, 8. Bulimina inflata Seguenza, 7: MPC-26172, 8: MPC-26173, both from sample MK01 (Yonahama F., PL2). 9, 10. Bulimina marginata d'Orbigny, 9: MPC-26176 from sample MK01 (Yonahama F., PL2); 10: MPC-26177 from sample MK02B (Minebari F., PL5). 11, 12. Bulimina striata d'Orbigny, 11: MPC-26178 from sample 3-2-low-silt (Onogoshi F., PL1); 12: MPC-26179 from sample 4-2 (Minebari F., PL5). 13, 14. Bulimina subornata Brady, 13: MPC-26180 from sample 5 (Minebari F., PL5); 14: MPC-26181 from sample MK02B (Minebari F., PL5). 15, 16. Bulimina truncana Gümbel, $15:$ MPC-26182 from sample 7 (Yonahama F., PL4); 16: MPC-26183 from sample 14-2 (Yonahama F., PL4). 17-19. Globobulimina pyrula (d'Orbigny), 17: MPC-26281 from sample 17 (Yonahama F., PL2); 18: MPC-26282 from sample MK02B (Minebari F., PL5); 19: MPC-26283 from sample 2-2 (Minebari F., PL5). 20. Buliminella elegantissima (d'Orbigny), MPC-26184 from sample MK02B (Minebari F., PL5). 21, 22. Euuvigerina asperula (Cžjžek), 21: MPC26243 from sample 8 (Yonahama F., PL4); 22: MPC-26244 from sample 16 (Yonahama F., PL3). 23, 24. Euuvigerina canariensis (d'Orbigny), 23: MPC-26245 from sample 2 (Minebari F., PL5); 24: MPC-26246 from sample MK02A (Minebari F., PL5). 
1953 Bulimina kochiensis Takayanagi, p. 31, pl. 4, fig. 12.

1966 Bulimina ampliapertula Belford, p. 65, pl. 5, figs. 18-21, text-figs. 5-10.

1975 Bulimina sp. - Kameyama, pl.-fig. 5.

1989 Bulimina kochiensis Takayanagi - Ōki, p. 114, pl. 11, fig. 2 (non Takayanagi, 1953).

1994 Bulimina ampliapertula Belford — Loeblich and Tappan, p. 124, pl. 243, figs. 11, 12; Akimoto, p. 283, pl. 2, fig. 10.

Description. Test elongate and slender, three times longer than wide; chambers inflated, increasing slowly as added; suture depressed; lower part of test ornamented with thin striae which form acute angles at periphery; test wall radiate, finely perforated; aperture narrow, slit-like, semi-vertical on apertural face; tooth plate developed.

Remarks. This species resembles the abovelisted taxa, yet is distinguished from them by its narrow apertural opening. In our view, Bulimina kochiensis is a senior synonym of $B$. ampliapertura.

Occurrence. Rare in the Minebari Formation.

\section{Bulimina marginata d'Orbigny}

Figures 26.9, 26.10

1826 Bulimina marginata d'Orbigny, p. 269, pl. 12, figs. 10-12.

1911 Bulimina marginata d'Orbigny - Cushman, pp. 83, 84, fig. 136.

1958 Bulimina marginata d'Orbigny - Asano, pp. 4-6, pl. 1, figs. 5, 9-11.

1964 Bulimina marginata d'Orbigny — LeRoy, p. F30, pl. 11, fig. 2.

1968 Bulimina marginata d'Orbigny — Huang, $p$. 56, pl. 13, fig. 33.

1987 Bulimina marginata d'Orbigny - Loeblich and Tappan, pl. 571, figs. 1-3.

1988 Bulimina marginata d'Orbigny - Wang, Zhang, Zhao, Min, Bian, Zheng, Cheng, and Chen, pp. 149, 150, pl. 34, figs. 1115; Marle, p. 141, pl. 2, fig. 8.

1989 Bulimina marginata d'Orbigny — Ōki, pp. 114, 115, 171, pl. 11, fig. 3; Inoue, p. 151, pl. 20, fig. 12, pl. 23, fig. 2, pl. 28, fig. 4 .

1990 Bulimina marginata d'Orbigny - Akimoto, p. 194 , pl. 16, fig. 6 .

1992 Bulimina marginata d'Orbigny - Ōki and Yamamoto, pp. 196, 197, fig. 4-13; Akimoto, p. 283, pl. 2, fig. 12.
1994 Bulimina marginata d'Orbigny - Jones, p. 55, pl. 51, figs. 3-5; Loeblich and Tappan, p. 124, pl. 242, figs. $1-4$.

1998 Bulimina marginata d'Orbigny - Hess, $p$. 76, pl. 10, fig. 7.

2000 Bulimina marginata d'Orbigny - Kim and Kucera, fig. 4.5.

2002 Bulimina marginata d'Orbigny - Akimoto, Matsui, Shimokawa, and Furukawa, p. 16, pl. 44, figs. 6, 7.

2006 Bulimina marginata d'Orbigny - Takata, Irizuki, and Ishida, pl. 1, fig. 5 .

Occurrence. Common in all the formations of the present study.

Bulimina striata d'Orbigny

Figures 26.11, 26.12

1826 Bulimina striata d'Orbigny, p. 269.

1944 Bulimina striata d'Orbigny - LeRoy (Part 1), p. 26, pl. 1, fig. 5.

1958 Bulimina striata d'Orbigny - Asano, p. 8, pl. 1, figs. 4, 7, 8 .

1988 Bulimina striata d'Orbigny - Marle, p. 141, pl. 2, fig. 10.

1989 Bulimina striata d'Orbigny - Inoue, p. 151, pl. 22, fig. 6, pl. 28, fig. 1, pl. 32, fig. 1.

1990 Bulimina striata d'Orbigny - Akimoto, p. 194, pl. 16, fig. 8, pl. 22, fig. 11; Ujiié, p. 31, figs. 9, 10.

1994 Bulimina striata d'Orbigny - Loeblich and Tappan, p. 125, pl. 242, figs. 8-14; Akimoto, p. 283, pl. 2, fig. 13.

1995 Bulimina striata d'Orbigny - Ujiié, p. 62, pl. 6, fig. 4.

2000 Bulimina striata d'Orbigny - Ohkushi, Thomas, and Kawahata, p. 139, pl. 2, fig. 3.

Occurrence. Common in the Onogoshi, Yonahama, and Minebari formations.

\section{Bulimina subornata Brady}

Figures 26.13, 26.14

1884 Bulimina subornata Brady, p. 402, pl. 51, fig. 6.

1900 Bulimina subornata Brady - Millett (part VIII), p. 276, pl. 2, fig. 3.

1911 Bulimina subornata Brady - Cushman, pp. 88, 89, fig. 141.

1958 Bulimina subornata Brady - Asano, p. 9, pl. 3, fig. 10. 
1966 Bulimina subornata Brady - Belford, pp. 64,65 , pl. 5, figs. $15-17$, text-figs. 5-11, 74, 7-5.

1989 Bulimina subornata Brady - Inoue, pl. 32, fig. 10.

1994 Bulimina subornata Brady - Jones, p. 55, pl. 51, fig. 6 .

Occurrence. Rare in the Yonahama and Minebari formations.

\section{Bulimina truncata Gümbel}

Figures 26.15, 26.16

1868 Bulimina truncana Gümbel, p. 644, pl. 2, fig. 77.

1884 Bulimina rostrata Brady, p. 408, pl. 51, figs. 14, 15.

1927 Bulimina alazanensis Cushman (b), p. 161, pl. 25, fig. 4.

1947 Bulimina truncana Gümbel - Cushman and Parker, p. 89, pl. 21, figs. 7, 8.

1947 Bulimina rostrata Brady - Cushman and Parker, p. 124, pl. 28, fig. 34.

1966 Bulimina alazanensis Cushman - Belford, pp. 62,63 , pl. 5, figs. 9-11, text-figs. 5-6, 7-6.

1977 Bulimina rostratiformis McCulloch, p. 245, pl. 104, fig. 8.

1988 Bulimina rostrata Brady - Wang, Zhang, Zhao, Min, Bian, Zheng, Cheng, and Chen, pp. 150, 151, pl. 21, figs. 9, 10.

1988 Bulimina alazanensis Cushman - Marle, p. 139, pl. 2, fig. 8.

1989 Bulimina truncana Guembel - Hermelin, p. 64 , pl. 12, figs. $1-3$.

1990 Bulimina rostrata Brady - Akimoto, p. 194, pl. 16, fig. 7.

1992 Bulimina rostrata Brady - Kaiho, pl. 4, fig. 5.

1994 Bulimina rostrata Brady - Jones, p. 56, pl. 51, figs. 14, 15.

1994 Bulimina rostratiformis McCulloch - Loeblich and Tappan, p. 124, pl. 239, figs. 11-14.

1995 Bulimina truncana Gümbel - Ujiié, pp. 62, 63 , pl. 6 , fig. 5 .

1998 Bulimina alazanensis Cushman - Hess, pl. 10, fig. 10.

2001 Bulimina truncana Gümbel - Hayward, Carter, Grenfell, and Hayward, figs. 14DD, 14-EE.
Remarks. Bulimina truncana and $B$. alazanensis were originally described from the Eocene; while $B$. rostrata is a Recent species. These three species are similar in having a compact, conical, truncated general morphology, but they can be subdivided into two morphospecies based on the number of longitudinal costae on the test surface. We generally agree with Ujiié's $(1990,1995)$ view of a wide range of variation in development of longitudinal costae; yet $B$. truncana Gümbel as illustrated by Ujiié (1990, pl. 12, fig. 8) shows numerous costae (about 10 are visible from one side), and this is considered to be out of the range of variation of $B$. truncana. Bulimina truncana has about five costae visible from one side.

Huang et al. (2007) reported abundant specimens of $B$. alazanensis from the Pliocene (mainly prior to $2.1 \mathrm{Ma}$ ) in the South China Sea, but did not illustrate any specimens. It might be the same species as $B$. truncana of the present study.

Occurrence. Common in the Yonahama Formation.

Genus GLOBOBULIMINA Cushman, 1927a Globobulimina pyrula (d'Orbigny)

Figures 26.17-26.19

1846 Bulimina pyrula d'Orbigny, p. 184, pl. 11, figs. 9, 10.

1911 Bulimina pyrula d'Orbigny - Cushman, p. 78, fig. 126.

1921 Bulimina pyrula d'Orbigny - Cushman, pp. 162, 163, fig. 1.

1921 Bulimina subaffinis Cushman, p. 166, fig. 7.

1947 Bulimina subaffinis Cushman - Cushman and Parker, p. 126, pl. 29, fig. 7.

1964 Bulimina subaffinis Cushman - LeRoy, p. F30, pl. 11, fig. 3.

1985 Bulimina pyrula d'Orbigny - Papp and Schmid, p. 69, pl. 62, figs. 8-10.

Remarks. Species of Globobulimina from Miyakojima Island converge with single species. Papp and Schmid (1985) discussed the taxonomy of Bulimina pupoides and Bulimina ovata, and concluded that they were junior synonyms of $B$. pyrula. All three species are known in Japan and have been distinguished as such. Thus, we cannot easily conclude that all of them are synonyms, because the discussion by Papp and Schmid (1985) is based solely upon general test shape. The inner structure, such as the tooth plate that has been emphasized in former studies, was not mentioned. 
Occurrence. Rare in the Minebari and Yonahama formations.

Family BULIMINELLIDAE Hofker, 1951 Genus BULIMINELLA Cushman, 1911 Buliminella elegantissima (d'Orbigny) Figure 26.20

1839 Bulimina elegantissima d'Orbigny (c), p. 51, pl. 7, figs. 13, 14

1987 Buliminella elegantissima (d'Orbigny) — Loeblich and Tappan, pl. 572, figs. 7-11.

1989 Buliminella elegantissima (d'Orbigny) Ōki, pp. 105, 170, pl. 8, fig. 8.

1994 Buliminella elegantissima (d'Orbigny) Jones, p. 55, pl. 50, figs. 20-22.

2002 Buliminella elegantissima (d7Orbigny) Akimoto, Matsui, Shimokawa, and Furukawa, p. 16, pl. 44, fig. 5.

Occurrence. Rare in the Minebari Formation.

Family UVIGERINIDAE Haeckel, 1894

Subfamily UVIGERININAE Haeckel, 1894

Genus EUUVIGERINA Thalmann, 1952

Euuvigerina asperula (Cžjžek)

Figures 26.21, 26.22

1848 Uvigerina asperula Cžjžek, p. 146, pl. 13, figs. 14, 15.

1866 Uvigerina hisipida Schwager, p. 249, pl. 7, fig. 95.

1913 Uvigerina asperula Czjzek - Cushman, $p$. 101, pl. 43, fig. 1.

1921 Uvigerina asperula Czjzek - Cushman, p. 274, pl. 54, fig. 5 .

1941 Uvigerina asperula Czjzek - LeRoy (Part 2), pp. 81, 82, pl. 2, fig. 16.

1964 Uvigerina hispida Schwager - LeRoy, p. F34, pl. 4, figs. 2, 3.

1968 Euuvigerina rustica (Cushman and Edwards) - Huang, pp. 56, 57, pl. 13, fig. 13 (non Uvigerina rustica Cushman and Edwards, 1938).

1990 Uvigerina hispida Schwager - Akimoto, $\mathrm{p}$. 215, pl. 16, fig. 11.

1990 Uvigerina asperula Czjzek - Ujiié, pp. 31, 32 , pl. 13, figs. 7,8 .

1995 Uvigerina asperula Czjzek - Ujiié, p. 63, pl. 6 , fig. 8.

Remarks. Here we follow Ujiié (1990) in distinguishing species with well-developed hispid tubercles from other hispid species such as Uvigerina auberiana d'Orbigny. The generic distinction between Euuvigerina and Uvigerina is based on the character of the inner structure of the apertural projection. This is occasionally difficult to observe in poorly preserved specimens.

Occurrence. Sporadic in the Yonahama Formation.

\section{Euuvigerina canariensis (d'Orbigny)}

Figures 26.23, 26.24

1839 Uvigerina canariensis d'Orbigny (b), p. 138, pl. 1, figs. 25-27.

1913 Uvigerina canariensis d'Orbigny - Cushman, pp. 92, 93, pl. 42, fig. 6 .

1988 Uvigerina canariensis d'Orbigny - Wang, Zhang, Zhao, Min, Bian, Zheng, Cheng, and Chen, p. 154, pl. 22, fig. 7, pl. 34, figs. 18-20. 25.

1990 Uvigerina proboscidea Schwager - Ujiié, p. 32, pl. 13, figs. 10, 11 (non Uvigerina proboscidea Schwager, 1866).

1994 Uvigerina canariensis d'Orbigny - Jones, p. 85 , pl. 74 , figs. $1-3$.

1994 Neouvigerina ampullacea (Schwager) Loeblich and Tappan, p. 126, pl. 246, figs. 9-19 (non Uvigerina asperula var. ampullacea Schwager, in Brady, 1884)

1995 Uvigerina canariensis d'Orbigny - Ujiié, p. 63, pl. 6, fig. 7 .

2001 Uvigerina canariensis d'Orbigny Kawagata, pp. 89, 91, fig. 9-3.

Remarks. Cushman (1913) distinguished Uvigerina canariensis from Uvigerina proboscidea mainly on the basis of the absence of long spines on the surface of the former. Such features, however, occasionally disappear in poorly preserved (abraded, etched, or recrystallized) specimens, making it difficult to distinguish these taxa.

Loeblich and Tappan's (1994) Neouvigerina ampullacea is not the same species as Jones's (1994) Siphouvigerina ampullacea, but is conspecific with $E$. canariensis. In the synonymy of Loeblich and Tappan's (1994) N. ampullacea, they included several $U$. proboscidea of previous papers, suggesting that many records of $U$. proboscidea in those papers possibly should be identified as $U$. canariensis.

Occurrence. Common in the Yonahama and Minebari formations.

\section{Euuvigerina vadescens (Cushman) \\ Figures 27.1, 27.2}

1933 Uvigerina proboscidea Schwager var. vadescens Cushman (b), p. 85, pl. 8, figs. 14, 15. 
1938 Uvigerina pseudoampullacea Asano (d), p. 613, pl. 17, figs. 28, 29.

1942 Uvigerina proboscidea Schwager var. vadescens Cushman - Cushman, pp. 50, 51, pl. 14, figs. 5-9.

1958 Uvigerina pseudoampullacea Asano Asano, p. 37, pl. 7, figs. 1, 2.

1964 Uvigerina proboscidea Schwager var. vadescens Cushman - LeRoy, p. F35, pl. 3, fig. 38.

1988 Uvigerina proboscidea Schwager - Marle, pl. 3, figs. 11, 12 (non Uvigerina proboscidea Schwager, 1866).

1989 Uvigerina proboscidea vadescens Cushman - Inoue, p. 153, pl. 20, fig. 2.

1989 Uvigerina vadescens Cushman - Ōki, pp. 120, 171, 172, pl. 12, fig. 5.

1990 Uvigerina proboscidea Schwager, vadescens Cushman - Ujiié, p. 32, pl. 13, fig. 9.

1994 Uvigerina proboscidea vadescens Cushman $-X u$ and Ujiié, p. 516, figs. 6-10.

1994 Uvigerina proboscidea Schwager Akimoto, p. 286, pl. 2, fig. 21.

2002 Uvigerina proboscidea vadescens Cushman - Akimoto, Matsui, Shimokawa, and Furukawa, p. 16, pl. 45, fig. 3.

Remarks. This species exhibits a wide range of variation in test size, length of apertural neck, and growth rate. It is occasionally difficult to distinguish younger specimens with shorter neck and compact chamber arrangement from $E$. canariensis, yet $E$. vadescens usually has a slender shape. There appear to be intermediate-transitional forms in the growth of elongate apertural neck and uncoiling mode of chambers between the present species and Siphouvigerina ampullacea (Brady).

Asano's (1958) data on the bathymetric distribution indicate that this species prefers the lower sublittoral to upper bathyal off southwest Japan where the warm Kuroshio water flows.

Occurrence. Common in the Oura and Onogoshi formations, rare in the Yonahama and Minebari formations.

Genus UVIGERINA d'Orbigny, 1826

Uvigerina flintii Cushman

Figures 27.3-27.5

1923 Uvigerina flintii Cushman, p. 165, pl. 42, fig. 13.

1966 Euuvigerina flintii (Cushman) - Belford, pp. 80,81 , pl. 7 , figs. $21-23$.
1994 Euuvigerina flintii (Cushman) - Loeblich and Tappan, p. 127, pl. 249, figs. 7-9.

Diagnosis. Moderate-sized species with inflated test and fine striae on the surface of chambers.

Remarks. Loeblich and Tappan (1994) assigned this species to the genus Euuvigerina attaching importance not on wall morphology (i.e., fine spines or longitudinal striations) but on the feature of the tooth plate inside the aperture. We rather attach importance to features of the wall surface. This is a tentative treatment, and emendation of the genus is needed.

Occurrence. Sporadic in the Onogoshi, Yonahama, and Minebari formations.

Uvigerina peregrina Cushman

Figures 27.6, 27.7

1923 Uvigerina peregrina Cushman, pp. 166, 167, pl. 42, figs. 7-10.

1948 Uvigerina peregrina Cushman var. dirupta Todd, in Cushman and McCullch, p. 267, pl. 34 , fig. 3.

1964 Uvigerina peregrina Cushman var. dirupta Todd - LeRoy, p. F34, pl. 4, fig. 4.

1966 Euuvigerina peregrina Cushman - Belford, pp. 75-77, pl. 7, figs. 3-7.

1968 Euuvigerina peregrina dirupta Todd Huang, p. 56, pl. 13, figs. 28, 29.

1987 Uvigerina peregrina Cushman - Loeblich and Tappan, pl. 573, figs. 24-27.

1988 Uvigerina peregrina Cushman - Marle, p. 149 , pl. 2, figs. 6,7 .

1989 Uvigerina peregrina Cushman - Hermelin, pp. 66,67 , pl. 12 , figs. $6,8$.

1990 Uvigerina peregrina Cushman - Ujiié, p. 31 , pl. 13 , figs. $1-3$.

1998 Uvigerina peregrina Cushman - Hess, p. 91, pl. 11, figs. 2, 3.

2000 Uvigerina peregrina Cushman - Ohkushi, Thomas, and Kawahata, p. 144, pl. 2, fig. 4.

Remarks. We do not discriminate the variety "dirupta" in the present study. Ujiie (1990) suggested that the growth of longitudinal costae discriminated dirupa from peregrina was an environmental effect.

Occurrence. Abundant in the Yonahama and Minebari formations.

\section{Uvigerina schencki Asano}

Figure 27.8

1950 Uvigerina schencki Asano (part 2), p. 12, text-figs. $74,75$. 


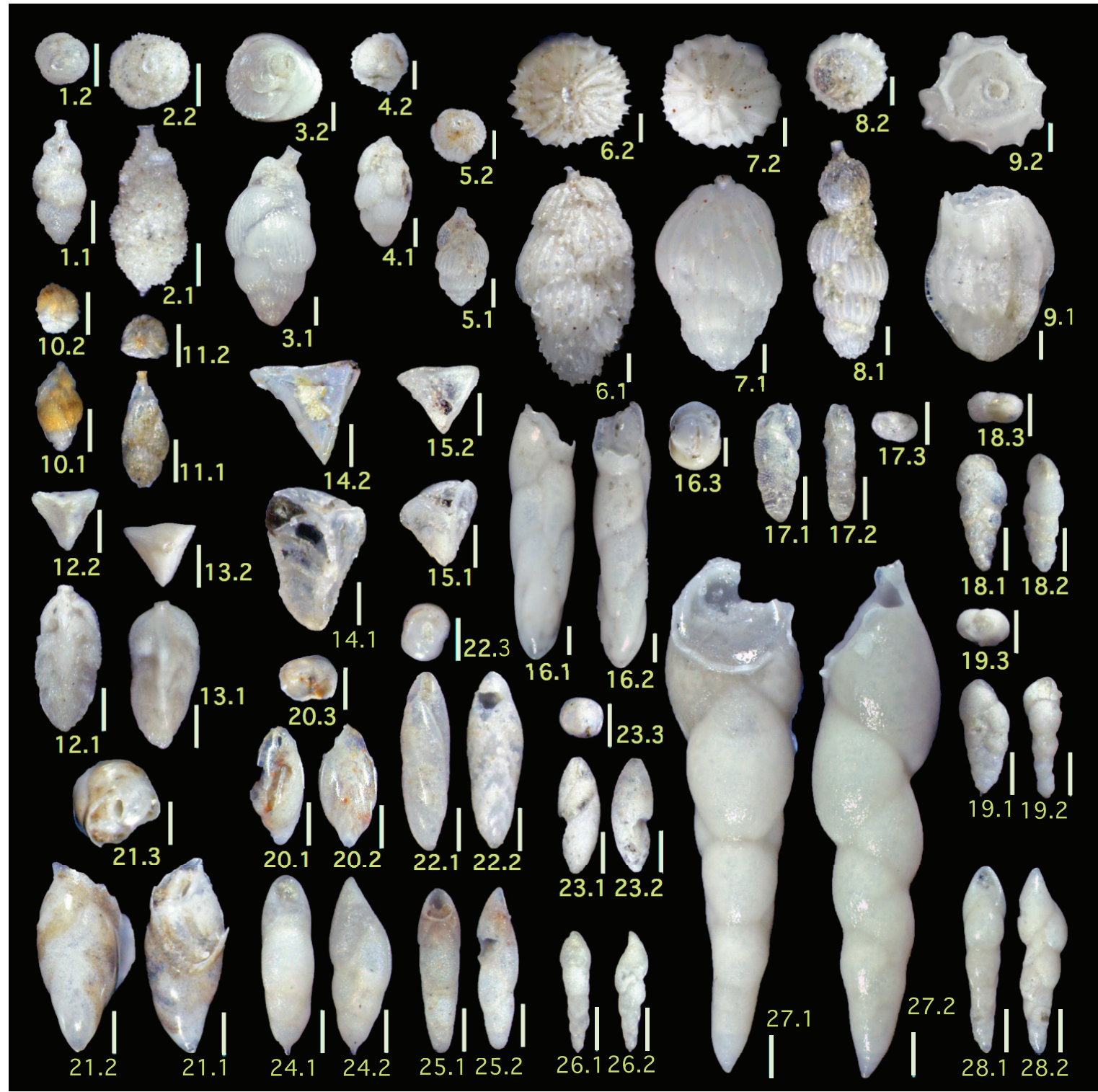

FIGURE 27. Family Uvigerinidae, Reussellidae, Fursenkoinidae, and Pleurostomellidae. All scale bars = 0.1mm. 1, 2. Euuvigerina vadescens (Cushman), 1: MPC-26247 from sample 10 (Onogoshi Formation, PL2); 2: MPC-26248 from sample 11 (Onogoshi F., ?PL1). 3-5. Uvigerina flintii Cushman, 3: MPC-26519 from sample 2-2 (Minebari F., PL5); 4: MPC-26520 from sample 3 (Onogoshi F., PL1); 5: MPC-26521 from sample 17 (Yonahama F., PL2). 6, 7. Uvigerina peregrina Cushman, 6: MPC-26522 from sample 6 (Yonahama F., PL2); 7: MPC-26523 from sample 6-2 (Yonahama F., PL2). 8. Uvigerina schencki Asano, MPC-26524 from sample 2 (Minebari F., PL5). 9. Uvigerina schwageri Brady, MPC-26525 from sample 5-2 (Minebari F., PL5). 10, 11. Angulogerina angulosa (Williamson), 10: MPC-26125 from sample MK01 (Yonahama F., PL2); 11: MPC-26126 from sample MK02B (Minebari F., PL5). 12, 13. Trifarina bradyi Cushman, 12: MPC-26517 from sample 3-2-up-sand (Onogoshi F., PL1); 13: MPC-26518 from sample 8 (Yonahama F., PL4). 14, 15. Reussella pulchra Cushman, 14: MPC-26461 from sample 2 (Minebari F., PL5); 15: MPC-26462 from sample MK01 (Yonahama F., PL2). 16. Fursenkoina acuta (d'Orbigny), MPC-26274 from sample 4-2 (Minebari F., PL5). 17-19. Neocassidulina hadai (Uchio), 17: MPC-26384 from sample 1 (Minebari F., PL5); 18: MPC-26385 from sample 15 (Yonahama F., PL3); 19: MPC-26386 from sample 16 (Yonahama F., PL3). 20, 21. Rutherfordoides mexicanus (Cushman), 20: MPC-26472 from sample 4 (Minebari F., PL5); 21: MPC-26473 from sample 5 (Minebari F., PL5). 22, 23. Rutherfordoides virgus (Nomura), 22: MPC-26474 from sample 1 (Minebari F., PL5); 23: MPC-26475 from sample 3-2-low-silt (Onogoshi F., PL1). 24, 25. Pleurostomella acuminata Cushman, 24: MPC-26420 from sample 7 (Yonahama F., PL4); 25: MPC-26421 from sample 17 (Yonahama F., PL2). 26-28. Pleurostomella alternans Schwager, 26: MPC-26422 from sample 7 (Yonahama F., PL4); 27: MPC-26423 and 28: MPC-26424 from sample 8-2 (Yonahama F., PL4); 27 large variation. 
1958 Uvigerina schencki Asano - Asano, pp. 37, 38, pl. 6, figs. 17, 18.

1988 Uvigerina schencki Asano - Wang, Zhang, Zhao, Min, Bian, Zheng, Cheng, and Chen, p. 154, pl. 22, fig. 8.

1989 Uvigerina schencki Asano - Ōki, pl. 12, fig. 4.

1990 Uvigerina schencki Asano - Akimoto, p. 215, pl. 16, fig. 13.

Occurrence. Two specimens from a single sample of the Minebari Formation.

\section{Uvigerina schwageri Brady}

Figure 27.9

1884 Uvigerina schwageri Brady, p. 575, pl. 74, figs. 8-10.

1913 Uvigerina schwageri Brady - Cushman, p. 97, pl. 37, figs. 3, 4 .

1921 Uvigerina schwageri Brady - Cushman, pl. 55, figs. 4, 5 .

1941 Uvigerina schwageri Brady — LeRoy (Part 1), p. 36, pl. 2, figs. 71, 72; LeRoy (Part 2), p. 82 , pl. 1, figs. $12,21$.

1958 Uvigerina schwageri Brady - Asano, p. 38 , pl. 6, figs. 1, 2.

1966 Euuvigerina schwageri (Brady) - Belford, pp. 81,82 , pl. 8 , figs. $1-5$.

1988 Uvigerina schwageri Brady - Wang, Zhang, Zhao, Min, Bian, Zheng, Cheng, and Chen, pp. 153, 154, pl. 22, fig. 6.

1992 Uvigerina schwageri Brady - Hatta and Ujié (b), p. 176, pl. 27, fig. 8.

1994 Uvigerina schwageri Brady - Jones, pp. 85, 86, pl. 74, figs. 8-10.

1994 Euuvigerina schwageri (Brady) — Loeblich and Tappan, p. 128, figs. 10-20.

1998 Uvigerina schwageri Brady - Hess, p. 92, pl. 11, fig. 1.

Occurrence. A single specimen from the Minebari Formation.

Subfamily ANGULOGERININAE Galloway, 1933

Genus ANGULOGERINA Cushman, 1927a Angulogerina angulosa (Williamson)

Figures 27.10, 27.11

1858 Uvigerina angulosa Williamson, p. 67, pl. 5, fig. 140.

1913 Uvigerina angulosa Williamson - Cushman, p. 98, pl. 44, fig. 4.
1964 Angulogerina japonica Asano - LeRoy, p. F35, pl. 5, fig. 24 (non Angulogerina japonica Asano, 1938d).

1988 Trifarina angulosa (Williamson) - Wang, Zhang, Zhao, Min, Bian, Zheng, Cheng, and Chen, p. 155, pl. 22, figs. 9, 10.

1988 Angulogerina angulosa (Williamson) Marle, p. 139, pl. 5, figs. 11, 12.

1989 Trifarina angulosa (Williamson) - Ōki, pp. 121, 122, p. 12, fig. 10.

1994 Trifarina angulosa (Williamson) - Jones, p. 86 , pl. 74, figs. 15, 16; Akimoto, p. 286, pl. 2, fig. 15; Xu and Ujiié, p. 516, fig. 6-12.

1994 Angulogerina angulosa (Williamson) Loeblich and Tappan, p. 128, pl. 250, figs. 13-20.

1995 Angulogerina angulosa (Williamson) Ujiié, pp. 63, 64, pl. 6, fig. 9.

2002 Angulogerina angulosa (Williamson) Akimoto, Matsui, Shimokawa, and Furukawa, p. 17, pl. 45, fig. 5 .

Occurrence. Common in the Onogoshi, Yonahama, and Minebari formations.

Genus TRIFARINA Cushman, 1923

Trifarina bradyi Cushman

Figures 27.12, 27.13

1923 Trifarina bradyi Cushman, p. 99, pl. 22, figs. 3-9.

1941 Trifarina bradyi Cushman — LeRoy (Part 1), p. 38, pl. 2, figs. 114, 115; LeRoy (Part 2), p. 82, pl. 2, fig. 24.

1942 Trifarina bradyi Cushman - Cushman, pp. 59, 60, pl. 15, fig. 13.

1958 Trifarina bradyi Cushman - Asano, pp. 40, 41, pl. 7, figs. 7, 8.

1959 Trifarina bradyi Cushman - Graham and Militante, pp. 88, 89, pl. 13, fig. 10.

1964 Trifarina bradyi Cushman - LeRoy, p. F35, pl. 3, figs. 17, 18.

1966 Trifarina bradyi Cushman - Belford, pp. 88, 89, pl. 9, figs. 16, 17.

1987 Trifarina bradyi Cushman - Loeblich and Tappan, pl. 574, figs. 10-13.

1988 Trifarina bradyi Cushman - Wang, Zhang, Zhao, Min, Bian, Zheng, Cheng, and Chen, p. 155, pl. 22, fig. 11; Marle, p. 149, pl. 5, fig. 10.

1990 Trifarina bradyi Cushman - Akimoto, p. 214, pl. 15, fig. 5 . 
1994 Trifarina bradyi Cushman - Jones, pp. 78, 79, pl. 67, figs. 1-3; Loeblich and Tappan, pp. 128, 129, pl. 251, figs. 6-16.

1998 Trifarina bradyi Cushman - Hess, p. 91, pl. 10, fig. 14.

2002 Trifarina bradyi Cushman - Hayward, p. 301, pl. 3, fig. 3; Akimoto, Matsui, Shimokawa, and Furukawa, p. 17, pl. 45, fig. 6.

Occurrence. Sporadic in all the formations of the present study.

Family REUSSELLIDAE Cushman, 1933c Genus REUSSELLA Galloway, 1933

Reussella pulchra Cushman

Figures 27.14, 27.15

1945 Reussella pulchra Cushman, p. 34, pl. 6, figs. 11, 12.

1994 Reussella pulchra Cushman - Loeblich and Tappan, p. 129, pl. 253, figs. 5-7.

Occurrence. Rare in the Onogoshi, Yonahama, and Minebari formations.

Superfamily FURSENKOINOIDEA Loeblich and Tappan, 1961

Family FURSENKOINIDAE Loeblich and Tappan, 1961

Genus FURSENKOINA Loeblich and Tappan, 1961

Fursenkoina acuta (d'Orbigny)

Figures 27.16

1846 Polymorphina acuta d'Orbigny, p. 234, pl. 13, figs. 4, 5, pl. 14, figs. 5-7.

1848 Virgulina schreibersiana Cžjžek, p. 147, pl. 13, figs. 18-23.

1911 Virgulina schreibersiana Czjzek - Cushman, p. 94, fig. 148.

1941 Virgulina squammosa d'Orbigny - LeRoy (Part 1), p. 33, pl. 2, figs. 24, 25 (non Virgulina squammosa d'Orbigny, 1826).

1942 Virgulina schreibersiana Czjzek - Cushman, pp. 12, 13, pl. 4, fig. 1.

1959 Virgulina schreibersiana Czjzek - Graham and Militante, p. 90, pl. 13, fig. 14.

1964 Virgulina schreibersiana Czjzek - LeRoy, p. F33, pl. 3, fig. 14.

1966 Fursenkoina schreibersiana (Czjzek) Belford, pp. 136, 137, pl. 9, figs. 18-21.

1985 Fursenkoina acuta (d'Orbigny) - Papp and Schmid, p. 82, pl. 75, figs. 1-6.

1987 Fursenkoina squammosa (d'Orbigny) Loeblich and Tappan, pl. 578, figs. 18-23.
1988 Fursenkoina schreibersiana (Czjzek) Wang, Zhang, Zhao, Min, Bian, Zheng, Cheng, and Chen, p. 173, pl. 30, fig. 5.

1989 Fursenkoina schreibersiana (Czjzek) Ōki, p. 142, pl. 18, fig. 5.

1993 Fursenkoina schreibersiana (Czjzek) Haig, pl. 5, figs. 18-20.

1994 Fursenkoina schreibersiana (Czjzek) Loeblich and Tappan, p. 131, pl. 257, figs. $1-12$.

1995 Fursenkoina schreibersiana (Czjzek) Ujié, p. 64, pl. 7, fig. 3.

1996 Fursenkoina schreibersiana (Czjzek) Revets, pp. 12, 13, pl. 8, figs. 5-8.

2001 Fursenkoina acuta (d'Orbigny) Kawagata, p. 92, fig. 9-8.

Remarks. We follow Revets's (1996a) discussion on the taxonomic relationship with $F$. squammosa, and Papp and Schmid (1985) and Kawagata (2001) with $F$. schreibersiana, granting priority to $F$. acuta.

Occurrence. A single specimen from the Minebari Formation.

Genus NEOCASSIDULINA McCulloch, 1977 Neocassidulina hadai (Uchio)

Figures 27.17-27.19

1962 Bolivina hadai Uchio, pp. 368, 369, pl. 18, figs. 3, 4.

1964 Bolivina capitata Cushman - LeRoy, p. F31, pl. 2, fig. 9 (non Bolivina capitata Cushman, 1933c).

1964 Loxostomum okinawaens LeRoy, p. F33, pl. 2, figs. 17, 18.

1979 Brizalina subcapitata Zheng, pp. 160, 218, pl. 15, fig. 15.

1989 Bolivina hadai Uchio - Ōki, p. 107, pl. 9, fig. 1.

1992 Brizalina capitata (Cushman) - Hatta and Ujiié (b), p. 172, pl. 25 figs. 9-11.

1993 Cassidelina capitata (Cushman) - Haig, p. 170, pl. 1, figs. 4-6.

1994 Cassidelina subcapitata (Zheng) - Loeblich and Tappan, p. 118, pl. 229, figs. 812.

Remarks. This species is commonly observed in the Pacific region as listed above. Bolivina capitata Cushman, which several authors have identified with the present form, has biserial chambers and a symmetrical aperture, and belongs to Bolivina or Brizalina according to Loeblich and Tappan (1994). 
Aperture of genus Neocassidulina is not symmetrical like Bolivina. Its elongate, curved, and slit-like aperture extends up the apertural face parallel to the distal margin in side view.

Occurrence. Sporadic in the Onogoshi, Yonahama, and Minebari formations.

Genus RUTHERFORDOIDES McCulloch, 1981

Rutherfordoides mexicanus (Cushman)

Figures 27.20, 27.21

1922 Virgulina mexicana Cushman (b), p. 120, pl. 23, fig. 8.

1958 Virgulina mexicana Cushman - Asano, pp. 14, 15, pl. 4, figs. 1, 2.

1983 Hastilina mexicana (Cushman) - Nomura (a), p. 82, pl. 2, fig. 7, pl. 3, fig. 4.

1987 Rutherfordoides mexicana (Cushman) Loeblich and Tappan, pl. 578, figs. 10-12.

1990 Rutherfordoides mexicanus (Cushman) Akimoto, p. 211, pl. 19, fig. 9.

1994 Rutherfordoides mexicanus (Cushman) Loeblich and Tappan, p. 131, pl. 257, figs. 13-15.

1995 Rutherfordoides rotundiformis (McCulloch) - Ujiié, p. 64, pl. 7, fig. 4 (non Rutherfordia rotundiformis McCulloch, 1977).

Remarks. Ujiie (1995) emphasized the similarity of his specimen to $R$. rotundiformis, though his specimen exhibited rapid growth in comparison to McCulloch's (1977) slender individual.

Occurrence. Rare in the Minebari Formation.

Rutherfordoides virgus (Nomura)

Figures 27.22, 27.23

1983 Hastilina virgula Nomura (a), p. 84, pl. 2, fig. 15.

1994 Rutherfordoides virgula (Nomura) - Loeblich and Tappan, p. 132, pl. 258, figs. 814.

Diagnosis. Moderate- to small-sized species with elongate-fusiform shape and smooth surface without significant depression of sutures and inflation of chambers.

Occurrence. A single specimen each from the Onogoshi Formation and the Minebari Formation.

Superfamily PLEUROSTOMELLOIDEA Reuss, 1860

Family PLEUROSTOMELLIDAE Reuss, 1860

Subfamily PLEUROSTOMELLINAE Reuss, 1860

Genus PLEUROSTOMELLA Reuss, 1860
Pleurostomella acuminata Cushman

Figures 27.24, 27.25

1922 Pleurostomella acuminata Cushman (b), pp. 50, 51, pl. 19, fig. 6 .

1989 Pleurostomella acuminata Cushman Hermelin, pp. 70, 71, pl. 13, fig. 1.

1992 Pleurostomella sp. B - Kaiho, pl. 5, fig. 5.

1994 Pleurostomella acuminata Cushman Jones, p. 56, pl. 51, fig. 22; Loeblich and Tappan, p. 133, pl. 261, figs. 11, 12.

2002 Pleurostomella acuminata Cushman Hayward, p. 302, pl. 2, figs. 20, 21.

2012 Pleurostomella acuminata Cushman Hayward, Kawagata, Sabaa, Grenfell, Kerckhoven, Johnson, and Thomas, p. 226, pl. 35, figs. 3-8.

Occurrence. Common in the Yonahama Formation, sporadic in the Minebari Formation.

Pleurostomella alternans Schwager

Figures 27.26-27.28

1866 Pleurostomella alternans Schwager, p. 238, fig. 79.

1911 Pleurostomella alternans Schwager Cushman, p. 50, fig. 81.

1941 Pleurostomella alternans Schwager LeRoy (Part 1), p. 38, pl. 3, figs. 54, 55; LeRoy (Part 2), p. 82, pl. 2, figs. 12, 13.

1964 Pleurostomella alternans Schwager LeRoy, p. F36, pl. 5, fig. 5.

1968 Pleurostomella alternans Schwager Huang, p. 59, pl. 12, fig. 4.

1994 Pleurostomella alternans Schwager Loeblich and Tappan, p. 133, pl. 261, figs. 13, 14.

1998 Pleurostomella alternans Schwager Hess, p. 86, pl. 11, fig. 10.

2002 Pleurostomella alternans Schwager Hayward, p. 302, pl. 2, figs. 22-24.

2012 Pleurostomella alternans Schwager Hayward, Kawagata, Sabaa, Grenfell, Kerckhoven, Johnson, and Thomas, p. 228, pl. 36, figs. 10-15.

Remarks. Small form (up to $0.5 \mathrm{~mm}$ in length) and large forms (ca. $1.1 \mathrm{~mm}$ ) are distinguished in samples from the Yonahama Formation. This gap in test size is large; forms might be distinguished as separate species.

Occurrence. Rare in the Yonahama and Minebari formations. 
Superfamily STILOSTOMELLOIDEA Finlay, 1947 Family STILOSTOMELLIDAE Finlay, 1947 Genus MYLLOSTOMELLA Hayward, 2002 Myllostomella fijiensis (Cushman)

Figures 28.1, 28.2

1934 Siphonodosaria fijiensis Cushman, p. 127, pl. 4, fig. 10.

1954 Ellipsonodosaria ugoensis Iwasa and Kikuchi, p. 192, text-fig. 5.

1992 Stilostomella cf. S. annulifera (Cushman and Bermudez) - Kaiho, pl. 5, fig. 15.

2002 Myllostomella fijiensis (Cushman) - Hayward, p. 304, pl. 3, figs. 10-12.

Remarks. Hayward (2002) proposed the new genus Myllostomella following a comprehensive summary by Loeblich and Tappan (1987); several species once assigned to Stilostomella or other genera were transferred to the new genus.

Occurrence. Common in the Yonahama Formation.

Genus NEUGEBORINA Popescu in Cicha, Rögl, Pupp, and Streiniger, 1998

Neugeborina longiscata (d'Orbigny)

Figures $10.13,10.14$

1846 Nodosaria longiscata d'Orbigny, p. 32, pl. 1, figs. $10-12$.

1941 Nodosaria longiscata d'Orbigny — LeRoy (Part 1), p. 27, pl. 3, fig. 69; LeRoy (Part 2), p. 75 , pl. 1, fig. 31.

1944 Nodosaria longiscata d'Orbigny - LeRoy (Part 2), p. 81, pl. 1, figs. 15, 16.

1964 Nodosaria longiscata d'Orbigny - LeRoy, p. F24, pl. 15, fig. 23.

1985 Nodosaria longiscata d'Orbigny - Papp and Schmid, p. 23, pl. 3, figs. 1-5.

1992 Nodosaria longiscata d'Orbigny - Kaiho, pl. 2, fig. 12.

1994 Nodosaria longiscata d'Orbigny - Loeblich and Tappan, pp. 65, 66, pl. 116, fig. 6; Akimoto, p. 285, pl. 4, fig. 6 .

2012 Neugeborina longiscata (d'Orbigny) Hayward, Kawagata, Sabaa, Grenfell, Kerckhoven, Johnson, and Thomas, pp. 134, 135, pl. 8, figs. 19-23.

Occurrence. Sporadic in the Yonahama and Minebari formations.
Genus ORTHOMORPHINA Stainforth, 1952

Orthomorphina perversa (Schwager)

Figure 28.5

1866 Nodosaria perversa Schwager, p. 212, pl. 5, fig. 29.

1884 Nodosaria perversa Schwager - Brady, p. 512, pl. 1, figs. 25-27.

1937 Nodogenerina challengeriana Thalmann, p. 341.

1964 Orthomorphina challengeriana (Thalmann) - LeRoy, p. F29, pl. 15, fig. 26.

1964 Rectoglandulina ambigua (Neugeboren) - LeRoy, p. F23, pl. 15, fig. 8 (non Nodosaria ambigua Neugeboren, 1856).

1989 ?Orthomorphina challengeriana (Thalmann) - Hermelin, p. 44, pl. 4, fig. 20.

1992 Stilostomella sp. G - Kaiho, pl. 5, fig. 23.

1994 Orthomorphina challengeriana (Thalmann) - Jones, p. 76, pl. 64, figs. 25-27, suppl. pl. 2, figs. 4, 5, 16.

2002 Orthomorphina perversa (Schwager) Hayward, p. 299, pl. 1, figs. 50-52.

2012 Orthomorphina perversa (Schwager) Hayward, Kawagata, Sabaa, Grenfell, Kerckhoven, Johnson, and Thomas, pp. 137, 138, pl. 8, figs. 35-38, pl. 9, figs. 1, 2,?3. ?4.

Remarks. This species is distinguished from Nodosaria ambigua Neugeboren by having welldeveloped longitudinal striae.

Occurrence. A single specimen from the Minebari Formation.

Genus SIPHONODOSARIA Silvestri, 1924

Siphonodosaria aff. consobrina (d'Orbigny) Figures 28.6, 28.7

Compared with:

1985 Stilostomella consobrina (d'Orbigny) Papp and Schmid, p. 29, pl. 11, figs. 1-5.

Remarks. The general morphology is similar to $S$. consobrina, but our specimens exhibit a well-developed apertural neck unlike the lectotype of Papp and Schmid (1985).

Occurrence. Four specimens from a single sample of the Yonahama Formation.

Siphonodosaria hayasakai (Ishizaki)

Figures 28.8, 28.9

1943 Ellipsonodosaria hayasakai Ishizaki, p. 683, pl., fig. 2. 


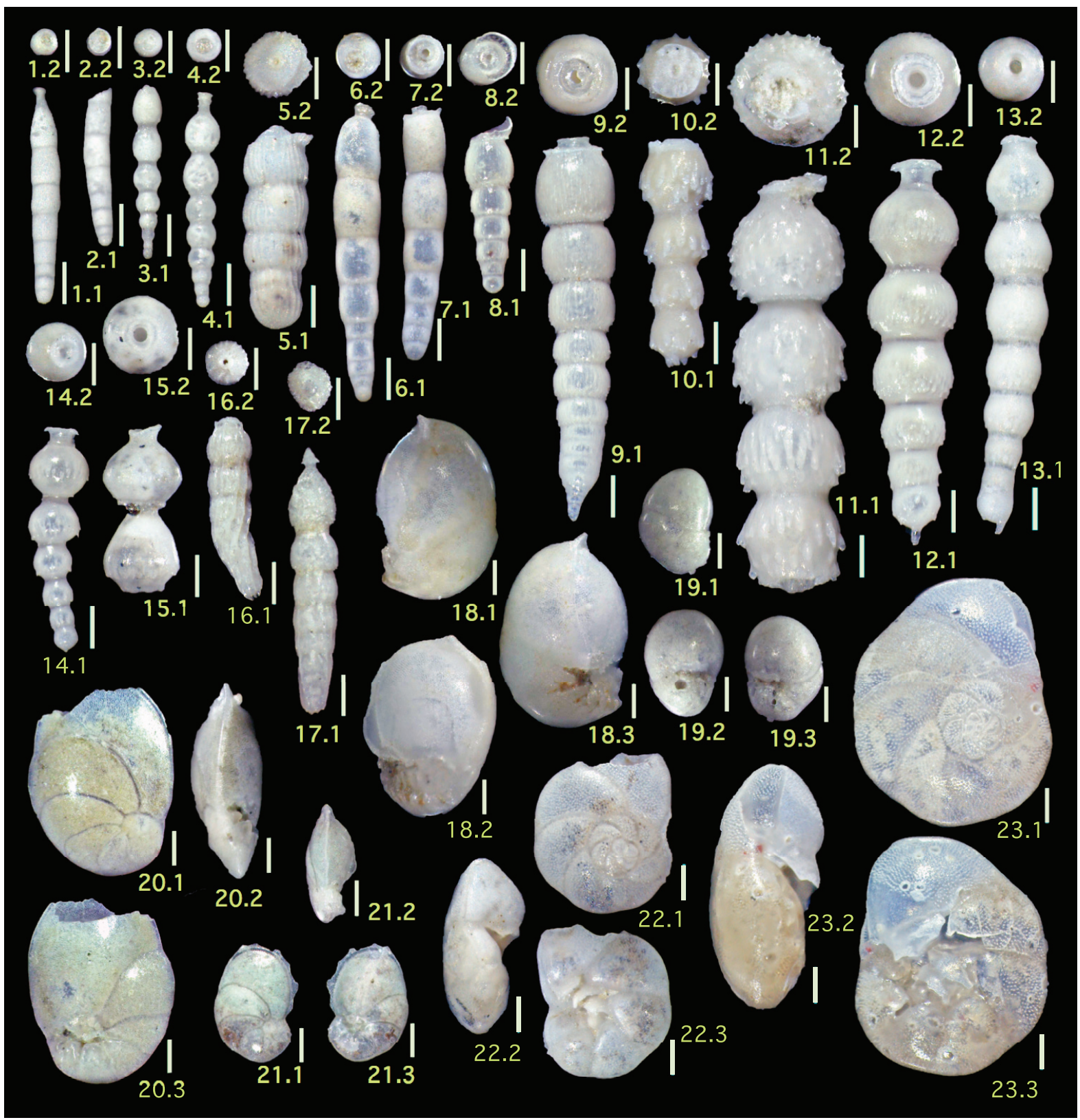

FIGURE 28. Family Stilostomellidae and Bagginidae. All scale bars $=0.1 \mathrm{~mm} .1$, 2. Myllostomella fijiensis (Cushman), 1: MPC-26382 from sample 7 (Yonahama Formation, PL4); 2: MPC-26383 from sample 15 (Yonahama F., PL3); $3,4$. Strictocostella hyugaensis (Ishizaki), 3: MPC-26513 from sample 3-2-low-silt (Onogoshi F., PL1); 4: MPC-26514 from sample 7 (Yonahama F., PL4). 5. Orthomorphina perversa (Schwager), MPC-26398 from sample 4-2 (Minebari F., PL5). 6, 7. Siphonodosaria aff. consobrina (d'Orbigny), 6: MPC-26495, 7: MPC-26496, both from sample 14-2 (Yonahama F., PL4). 8, 9. Siphonodosaria hayasakai (Ishizaki), 8: MPC-26497 from sample 4 (Minebari F., PL5); 9: MPC26498 from sample 6 (Yonahama F., PL2). 10, 11. Siphonodosaria longispina (Egger), 10: MPC-26499 from sample 8 (Yonahama F., PL4); 11: MPC-26500 from sample 8-2 (Yonahama F., PL4). 12, 13. Siphonodosaria ketienziensis (Ishizaki), 12: MPC-26501 from sample 14 (Yonahama F., PL4); 13: MPC-26502 from sample 14-2 (Yonahama F., PL4). 14, 15. Siphonodosaria lepidula (Schwager), 14: MPC-26503 from sample 5 (Minebari F., PL5); 15: MPC-26504 from sample MK01 (Yonahama F., PL2). 16, 17. Strictocostella modesta (Bermudez), 16: MPC-26515 from sample 14 (Yonahama F., PL4); 17: MPC-26516 from sample 16 (Yonahama F., PL3); 16, apertural projection broken. $18,19$. Baggina totomiensis Makiyama, 18: MPC-26138 from sample 3 (Onogoshi F., PL1); 19: MPC-26139 from sample 11 (Onogoshi F., ?PL1). 20, 21. Cancris auriculus (Fichtel and Moll), 20: MPC-26187 from sample 2 (Minebari F., PL5); 21: MPC-26188 from sample 18 (Oura F., ?PL1). 22, 23. Valvulineria glabra Cushman, 22: MPC-26531 from sample MK02B (Minebari F., PL5); 23: MPC-26532 from sample 14-2 (Yonahama F., PL4). 
Diagnosis. Moderate-sized species with very finely hispid test surface, slightly inflated test, and depressed sutures.

Occurrence. Rare in the Yonahama Formation, common in the Minebari Formation.

Siphonodosaria longispina (Egger)

Figures 28.10, 28.11

1900 Nodosaria longispina Egger, p. 80, pl. 10, fig. 22.

1964 Nodosaria hispidula Cushman - LeRoy, p. F24, pl. 15, fig. 4 (non Nodosaria lepidula Schwager var. hispidula Cushman, 1921).

2002 Siphonodosaria hispidula (Cushman) Hayward, p. 304, pl. 3, figs. 18 (only).

2012 Siphonodosaria longispina (Egger) - Hayward, Kawagata, Sabaa, Grenfell, Kerckhoven, Johnson, and Thomas, p. 177, pl. 18 , figs. $28-30$.

Remarks. As indicated by Hayward et al. (2012), this species differs from $N$. hispidula in having welldeveloped long spines on the test.

Occurrence. Rare in the Yonahama and Minebari formations.

\section{Siphonodosaria ketienziensis (Ishizaki)}

Figures 28.12, 28.13

1943 Ellipsonodosaria ketienziensis Ishizaki, p. 684, figs. 1, 6, 11.

1964 Stilostomella ketienziensis (Ishizaki) LeRoy, p. F35, pl. 15, fig. 33.

1992 Stilostomella sp. A - Kaiho, pl. 5, fig. 11.

2002 Siphonodosaria ketienziensis (Ishizaki) Hayward, pp .304, 305, pl. 3, figs. 21, 22.

Remarks. This species resembles Siphonodosaria lepidula, yet is distinguished by less depressed sutures and finer and shorter spines on the lower margin of the chambers. Nodosaria insecta var. spinifera LeRoy (1941, part 2, p. 74, pl. 1, fig. 17) may be a synonym of this species. Hayward et al. (2012) regarded this species to be synonymous with Siphonodosaria jacksonensis (Cushman and Applin), originally described from the Eocene of Texas, although retained as a distinct species, since the holotype illustrated by Hayward et al. shows less depressed sutures.

Occurrence. Common in the Onogoshi, Yonahama, and Minebari formations.

Siphonodosaria lepidula (Schwager)

Figures 28.14, 28.15

1866 Nodosaria lepidula Schwager, pp. 210, 211, pl. 5, figs. 27, 28.
1941 Nodogenerina lepidula (Schwager) LeRoy (Part 1), p. 31, pl. 1, fig. 104; LeRoy (Part 2), p. 79, pl. 1, fig. 28.

1989 Siphonodosaria lepidula (Schwager) Hermelin, pp. 61, 62, pl. 11, figs. 8, 9.

1992 Stilostomella lepidula (Schwager) Kaiho, pl. 5, fig. 12.

1994 Nodogenerina lepidula (Schwager) - Loeblich and Tappan, p. 133, pl. 261, figs. 18-20.

2002 Siphonodosaria lepidula f. lepidula (Schwager) - Hayward, p. 305, pl. 3, figs. 25-32.

2012 Siphonodosaria lepidula (Schwager) Hayward, Kawagata, Sabaa, Grenfell, Kerckhoven, Johnson, and Thomas, p. 174, pl. 18, figs. $6-20$.

Remarks. This is one of the most popular deepsea foraminifera in the Neogene Pacific region, and its disappearance provides an important datum horizon in the Quaternary which is referred to as the Stilostomella extinction (Weinholz and Lutze, 1989).

Occurrence. Common in the Onogoshi, Yonahama, and Minebari formations.

Genus STRICTOCOSTELLA Patterson, 1987 Strictocostella hyugaensis (Ishizaki)

Figures 28.3, 28.4

1943 Ellipsonodosaria hyugaensis Ishizaki, p. 686, text-figs. 12, 13.

2005 Myllostomella hyugaensis (Ishizaki) Kawagata, Hayward, Grenfell, and Sabaa, p. 288, pl. 1, figs. 12, 13.

2012 Strictocostella hyugaensis (Ishizaki) Hayward, Kawagata, Sabaa, Grenfell, Kerckhoven, Johnson, and Thomas, pp. 188, 189, pl. 21, figs. $27-32$, pl. 22, figs. $1-4$.

Occurrence. Common in the Yonahama Formation, rare in the Onogoshi and Minebari formations.

Strictocostella modesta (Bermudez)

Figures 28.16, 28.17

1937 Ellipsonodosaria modesta Bermudez, p. 238, pl. 20, fig. 3.

1989 Siphonodosaria sp. 3 - Hermelin, p. 62, pl. 11, figs. 15, 19.

2002 Strictocostella modesta (Bermudez) Hayward, p. 306, pl. 3, figs. 51, 52.

2002 Strictocostella modesta (Bermúdez) Hayward, Kawagata, Sabaa, Grenfell, Kerckhoven, Johnson, and Thomas, pp. 191, 192, pl. 22, figs. 28-39. 
Occurrence. Common in the Yonahama Formation.

Superfamily DISCORBOIDEA Ehrenberg, 1838

Family BAGGINIDAE Cushman, 1927a

Subfamily BAGGININAE Cushman, 1927a

Genus BAGGINA Cushman, 1926b

Baggina totomiensis Makiyama

Figure 28.18, 28.19

1931 Baggina totomiensis Makiyama, p. 42, text-fig. 4

1951 Baggina totomiensis Makiyama - Asano (part 14), p. 21, text-figs. 154, 155

1964 Baggina totomiensis Makiyama - LeRoy, p. F39, pl. 6, figs. 20-22.

Remarks. This species is characterized by the dentition on the early chambers in the umbilical region. It resembles the Miocene Baggina notoensis Asano (1953) in a dentate umbilicus, but is distinguished by its evolute dorsal chambers.

Occurrence. Rare in the Onogoshi, Yonahama, and Minebari formations.

Genus CANCRIS de Montfort, 1808

Cancris auriculus (Fichtel and Moll)

Figures 28.20, 28.21

1798 Nautilus auriculata var. $\alpha$ and $\beta$ Fichtel and Moll, pp. 108, 110, pl. 20, figs. a-f.

1921 Pulvinulina auricula (Fichtel and Moll) Cushman, p. 329, pl. 69, fig. 3.

1941 Cancris auriculus (Fichtel and Moll) LeRoy (Part 1), p. 41, pl. 2, figs. 79-81; LeRoy (Part 3), p. 117, pl. 3, figs. 7-9, 16-18.

1944 Cancris auriculus (Fichtel and Moll) LeRoy (Part 1), p. 36, pl. 3, figs. 4-9, pl. 6 , figs. $16-18$.

1964 Cancris auriculus (Fichtel and Moll) LeRoy, p. F39, pl. 4, figs. 23, 24.

1965 Cancris auriculus (Fichtel and Moll) Todd, p. 22, pl. 5, fig. 5.

1966 Cancris auriculus (Fichtel and Moll) - Belford, pp. 96,97 , pl. 15, figs. $1-5$.

1984 Cancris auriculus (Fichtel and Moll) Rögl and Hansen, pp. 67,68 , pl. 26, figs. 3-8, text-fig. 28.

1985 Cancris auriculus (Fichtel and Moll) Papp and Schmid, p. 61, pl. 52, figs. 713.

1988 Cancris auriculus (Fichtel and Moll) Wang, Zhang, Zhao, Min, Bian, Zheng, Cheng, and Chen, p. 158, pl. 23, fig. 13.
1989 Cancris auriculus (Fichtel and Moll) Inoue, pl. 30, fig. 6.

1989 Cancris auricula (Fichtel and Moll) - Ōki, pp. 128, 129, pl. 14, fig. 8.

1992 Cancris auriculus (Fichtel and Moll) Hatta and Ujiié (b), p. 179, pl. 29, fig. 4.

1994 Cancris auriculus (Fichtel and Moll) Jones, p. 105, pl. 106, fig. 4; Loeblich and Tappan, p. 134, pl. 265, figs. 7-10.

1996 Cancris auriculus (Fichtel and Moll) Revets (b), pp. 76, 77, pl. 10, figs. 5-8.

2001 Cancris auriculus (Fichtel and Moll) Kawagata, pp. 92, 93, figs. 9, 10.

2002 Cancris auriculus (Fichtel and Moll) Akimoto, Matsui, Shimokawa, and Furukawa, p. 17, pl. 47, fig. 2.

Occurrence. Sporadic in the Oura, Onogoshi, and Minebari formations.

Genus VALVULINERIA Cushman, 1926b Valvulineria glabra Cushman

Figures 28.22, 28.23

1927 Valvulineria vilardeboana (d'Orbigny) var. glabra Cushman (c), p. 161, pl. 9, figs. 5, 6.

1941 Valvulineria aff. araucana (d'Orbigny) var. malagaensis Kleinpell - LeRoy (Part 2), p. 83, pl. 4, figs. 22-24.

1944 Valvulineria araucana (d'Orbigny) var. malagaensis Kleinpell - LeRoy (Part 1), p. 33, pl. 6, figs. 28-30.

1965 Valvulineria glabra Cushman - Todd, p. 22, pl. 8, fig. 3.

1994 Valvulineria glabra Cushman - Loeblich and Tappan, p. 135, pl. 268, figs. 1-3.

Occurrence. Sporadic in the Yonahama Formation, rare in the Minebari Formation.

Family DISCORBIDAE Ehrenberg, 1838

Genus ROTORBIS Sellier de Civrieux, 1977 Rotorbis auberi (d'Orbigny)

Figures 29.1, 29.2

1839 Rosalina auberi d'Orbigny (a), p. 94, pl. 4, figs. 5-8.

1987 Neoeponides auberi (d'Orbigny) - Loeblich and Tappan, pl. 605, figs. 5-7.

1994 Neoeponides auberi (d'Orbigny) - Jones, p. 94 , pl. 87, fig. 8 .

1994 Rotorbis auberi (d'Orbigny) - Loeblich and Tappan, pp. 137, 138, pl. 278, figs. 111. 
2001 Neoeponides auberi (d'Orbigny) Kawagata, p. 93, figs. 9-11.

Remarks. Loeblich and Tappan (1987) once placed the genus Rotorbis in the synonymy of Neoeponides. Later, Loeblich and Tappan (1994) regarded it as a distinct genus of the family Discorbidae Ehrenberg (1838), and the genus Neoeponides was placed in a new family, Neoeponididae.

Occurrence. Common in the Minebari Formation.

Genus STREBLOIDES Bermúdez and Seiglie, 1963

Strebloides advenus (Cushman)

Figure 29.5

1922 Discorbis advena Cushman (a), p. 40.

1959 Discopulvinulina advena (Cushman) Graham and Militante, pp. 92, 93, pl. 13, fig. 21.

1987 Strebloides advenus (Cushman) - Loeblich and Tappan, pl. 608, figs. 1-3.

1994 Strebloides advenus (Cushman) - Jones, p. 93, pl. 87, fig. 1.

Occurrence. A single specimen from the Yonahama Formation.

Family NEOEPONIDIDAE Loeblich and Tappan, 1994

Genus NEOEPONIDES Reiss, 1960 Neoeponides bradyi Le Calvez

Figures 29.3, 29.4

1974 Neoeponides bradyi Le Calvez, p. 64.

1984 Neoeponides bradyi Le Calvez - Rögl and Hansen, pl. 7, figs. 1-6.

1994 Neoeponides bradyi Le Calvez — Loeblich and Tappan, p. 138, pl. 279, figs. 1-9.

Occurrence. Rare in the Yonahama Formation, sporadic in the Minebari Formation.

Family ROSALINIDAE Reiss, 1963

Genus GAVELINOPSIS Hofker, 1951

Gavelinopsis lobatulus (Parr)

Figures 29.6, 29.7

1950 Discorbis lobatulus Parr, p. 354, pl. 13, figs. 23-25.

1988 Gavelinopsis lobatulus (Parr) - Marle, p. 143, pl. 2, figs. $1-3$.

1989 Gavelinopsis lobatulus (Parr) - Hermelin, p. 68, pl. 12, figs. 11-13.

1992 Gavelinopsis lobatulus (Parr) - Kaiho, pl. 7, fig. 5.

1994 Gavelinopsis labatula (Parr) - Jones, p. 94, pl. 88, fig. 1.
Occurrence. Common in the Yonahama Formation, rare in the Minebari Formation.

Gavelinopsis praegeri (Heron-Allen and Earland)

Figures 29.8, 29.9

1913 Discorbina praegeri Heron-Allen and Earland, p. 122, pl. 10, figs. 8-10.

1941 Discorbis? cf. praegeri (Heron-Allen and Earland) - LeRoy (Part 3), p. 116, pl. 2, figs. 7-9.

1987 Gavelinopsis praegeri (Heron-Allen and Earland) - Loeblich and Tappan, pl. 608, figs. 6-12.

1988 Gavelinopsis praegeri (Heron-Allen and Earland) - Wang, Zhang, Zhao, Min, Bian, Zheng, Cheng, and Chen, p. 157, pl. 23, figs. 7-11.

1990 Gavelinopsis praegeri (Heron-Allen and Earland) - Ujiié, p. 33, pl. 14, fig. 6.

1994 Gavelinopsis praegeri (Heron-Allen and Earland) - Loeblich and Tappan, p. 138, 139, pl. 281, figs. 1-10; Akimoto, p. 284, pl. 3, fig. 14.

2001 Gavelinopsis praegeri (Heron-Allen and Earland) - Hayward, Carter, Grenfell, and Hayward, figs. 15-BB-15-DD.

Occurrence. Common in the Oura and Onogoshi formations, rare in the Yonahama Formation.

Genus PLANODISCORBIS Bermúdez, 1952 Planodiscorbis rarescens (Brady)

Figures 29.10, 29.11

1884 Discorbina rarescens Brady, p. 651, pl. 90, figs. 2, 3.

1987 Planodiscorbis rarescens (Brady) - Loeblich and Tappan, pl. 609, figs. 11-13.

1990 Planodiscorbis rarescens (Brady) - Ujiié, pp. 33, 34, pl. 13, fig. 12.

1992 Planodiscorbis rarescens (Brady) - Hatta and Ujiié (b), p. 183, pl. 32, fig. 4.

1994 Planodiscorbis rarescens (Brady) Jones, p. 95, pl. 90, figs. 2, 3; Loeblich and Tappan, pp. 139, 140, pl. 285, figs. 1-10.

1995 Planodiscorbis rarescens (Brady) - Ujiié, p. 66 , pl. 8 , fig. 8.

Occurrence. Rare in the Onogoshi and Yonahama formations, common in the Minebari Formation.

Genus ROSALINA d'Orbigny, 1826

Rosalina australis (Parr)

Figure 29.12

1932 Discorbis australis Parr (b), p. 227. 


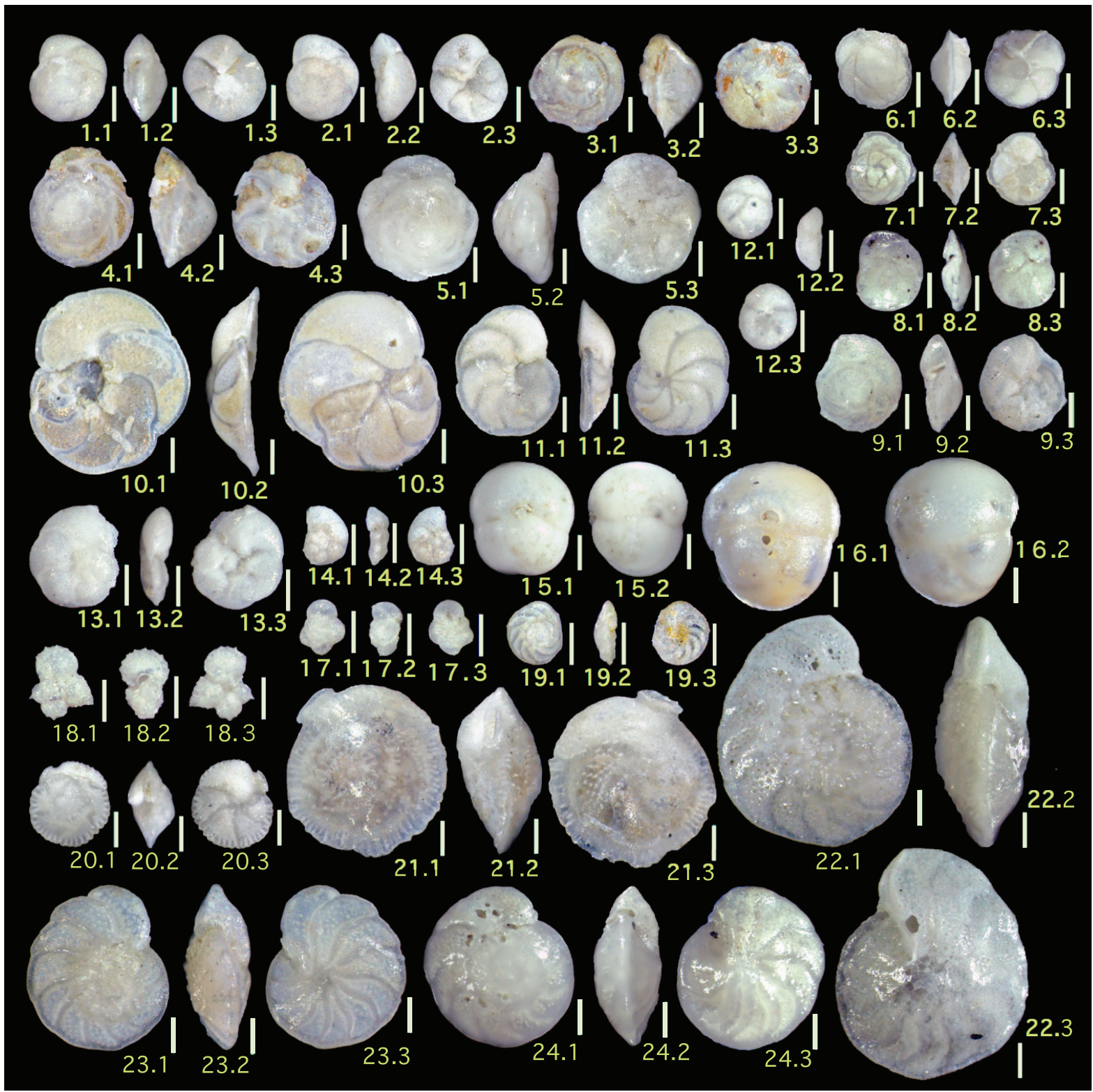

FIGURE 29. Family Discorbidae, Rosalinidae, Sphaeroidinidae, Glabratellidae, Siphonidae, and Parrelloididae. All scale bars $=0.1 \mathrm{~mm}$. 1, 2. Rotorbis auberi (d'Orbigny), 1: MPC-26470 from sample 4 (Minebari Formation, PL5); 2: MPC-26471 from sample 12 (Minebari F., PL5). 3, 4. Neoeponides bradyi Le Calvez, 3: MPC-26387 from sample 2-2 (Minebari F., PL5); 4: MPC-26388 from sample 6-2 (Yonahama F., PL2). 5. Strebloides advenus (Cushman), MPC26512 from sample 8-2 (Yonahama F., PL4). 6, 7. Gavelinopsis lobatulus (Parr), 6: MPC-26275 from sample 15 (Yonahama F., PL3); 7: MPC-26276 from sample MK01 (Yonahama F., PL2). 8, 9. Gavelinopsis praegeri (Heron-Allen and Earland), 8: MPC-26277 from sample 11 (Onogoshi F., ?PL1); 9: MPC-26278 from sample 18 (Oura F., ?PL1). $10,11$. Planodiscorbis rarescens (Brady), 10: MPC-26411 from sample 2 (Minebari F., PL5); 11: MPC-26412 from sample 3-2up-sand (Onogoshi F., PL1). 12. Rosalina australis (Parr), MPC-26463 from sample MK01 (Yonahama F., PL2). 13, 14. Rosalina globularis d'Orbigny, 13: MPC-26464 from sample 5-2 (Minebari F., PL5); 14: MPC-26465 from sample MK01 (Yonahama F., PL2). 15, 16. Sphaeroidina bulloides d'Orbigny, 15: MPC-26505 from sample 3-2-low-silt (Onogoshi F., PL1); 16: MPC-26506 from sample MK01 (Yonahama F., PL2). 17, 18. Murrayinella globosa (Millett), 17: MPC-26380 from sample 1 (Minebari F., PL5); 18: MPC-26381 from sample 14-2 (Yonahama F., PL4). 19. Planoglabratella opercularis (d'Orbigny), MPC-26413 from sample MK02B (Minebari F., PL5), juvenile specimen. $20,21$. Siphonina australis Cushman, 20: MPC-26493 from sample 15 (Yonahama F., PL3); 21: MPC-26494 from sample MK01 (Yonahama F., PL2). 22-24. Cibicidoides ornatus (Cushman), 22: MPC-26200 from sample 6 (Yonahama F., PL2); 23: MPC-26201 from sample MK01 (Yonahama F., PL2); 24: MPC-26202 from sample 4-2 (Minebari F., PL5). 
1951 Discopulvinulina australis (Parr) - Asano (part 14), p. 3, text-figs. 20-22.

1988 Rosalina australis (Parr) - Wang, Zhang, Zhao, Min, Bian, Zheng, Cheng, and Chen, p. 156, pl. 22, figs. 14, 15.

1994 Rosalina australis (Parr) - Jones, p. 94, pl. 87, figs. 5-7.

2002 Rosalina australis (Parr) - Akimoto, Matsui, Shimokawa, and Furukawa, p. 18, pl. 48, fig. 8.

Occurrence. A single specimen from the Yonahama Formation.

Rosalina globularis d'Orbigny

Figures 29.13, 29.14

1826 Rosalina globularis d'Orbigny, p. 271, pl. 13 , figs. $1-4$.

1965 Rosalina globularis d'Orbigny - Todd, pp. 11, 12, pl. 3, fig. 4 .

1987 Rosalina globularis d'Orbigny - Loeblich and Tappan, pl. 610, figs. $1-5$, pl. 611 , figs. $1-3$.

1994 Rosalina globularis d'Orbigny - Jones, $p$. 93, pl. 86, fig. 13; Loeblich and Tappan, p. 140 , pl. 286, figs. $7-15$.

2002 Rosalina globularis d'Orbigny - Akimoto, Matsui, Shimokawa, and Furukawa, p. 18, pl. 49 , fig. 6 .

Occurrence. Rare in the Yonahama and Minebari formations.

Family SPHAEROIDINIDAE Cushman, 1927a

Genus SPHAEROIDINA d'Orbigny, 1826

Sphaeroidina bulloides d'Orbigny

Figures 29.15, 29.16

1826 Sphaeroidina bulloides d'Orbigny, p. 267.

1846 Sphaeroidina austriaca d'Orbigny, p. 284, pl. 20, figs. 19-21.

1865 Sphaeroidina bulloides d'Orbigny Parker, Jones, and Brady, p. 29, pl. 2, fig. 58.

1941 Sphaeroidina bulloides d'Orbigny LeRoy (Part 1), p. 43, pl. 1, figs. 11-13; LeRoy (Part 2), p. 86, pl. 6, figs. 5, 6.

1944 Sphaeroidina bulloides d'Orbigny LeRoy (Part 1), pp. 38, 39, pl. 4, figs. 49, 50; LeRoy (Part 2), p. 90, pl. 3, figs. 24, 25.

1951 Sphaeroidina austriaca d'Orbigny Asano (part 12), p. 12, text-figs. 13, 14.

1957 Sphaeroidina bulloides d'Orbigny Asano, p. 10, pl. 2, figs. 16, 17.
1964 Sphaeroidina bulloides d'Orbigny LeRoy, p. F41, pl. 16, figs. 21, 22.

1965 Sphaeroidina bulloides d'Orbigny - Todd, p. 49 , pl. 18, fig. 4 .

1968 Sphaeroidina bulloides d'Orbigny Huang, p. 59, pl. 10, figs. 5, 10.

1985 Sphaeroidina bulloides d'Orbigny - Papp and Schmid, p. 96, pl. 90, figs. 7-12.

1987 Sphaeroidina bulloides d'Orbigny - Loeblich and Tappan, pl. 617, figs. 1-6.

1988 Sphaeroidina bulloides d'Orbigny Wang, Zhang, Zhao, Min, Bian, Zheng, Cheng, and Chen, p. 146, pl. 20, fig. 6; Marle, p. 149, pl. 2, fig. 11.

1989 Sphaeroidina bulloides d'Orbigny — Inoue, p. 149, pl. 27, fig. 9.

1990 Sphaeroidina bulloides d'Orbigny — Ujiié, pp. 28, 29, pl. 11, figs. 3-5.

1994 Sphaeroidina bulloides d'Orbigny Jones, p. 91, pl. 84, figs. 1-7; Loeblich and Tappan, p. 141, pl. 289, figs. 1-3.

1998 Sphaeroidina bulloides d'Orbigny - Hess, p. 90, pl. 9, fig. 14

2001 Sphaeroidina bulloides d'Orbigny - Hayward, Carter, Grenfell, and Hayward, fig. 16-BB.

Remarks. Papp and Schmid (1985) regarded S. austriaca, from the Miocene, as a junior synonym of $S$. bulloides. We think that Japanese specimens recorded as S. austriaca (e.g., Asano, 1951, part $12)$ are also junior synonyms of $S$. bulloides.

Occurrence. Common in the Onogoshi, Yonahama, and Minebari formations.

Superfamily GLABRATELLOIDEA Loeblich and Tappan, 1964

Family GLABRATELLIDAE Loeblich and Tappan, 1964

Genus MURRAYINELLA Farías, 1977 Murrayinella globosa (Millett)

Figures 29.17, 29.18

1903 Discorbina imperatorial (d'Orbigny) var. globosa Millett (part VII), p. 701, pl. 7, fig. 6.

1988 Schackoinella globosa (Millett) - Wang, Zhang, Zhao, Min, Bian, Zheng, Cheng, and Chen, p. 158, pl. 23, figs. 15, 16.

1989 Pararotalia aff. globosa (Millett) - Ōki, p. 133, pl. 15, fig. 9. 
1994 Schackoinella globosa (Millett) - Loeblich and Tappan, p. 142, pl. 294, figs. 110.

2000 Murrayinella globosa (Millett) - Nomura and Takayanagi, pp. 174, 175, figs. 1.11.8.

Remarks. Specimens from Miyakojima Island have four to four and a half chambers in the final whorl rather than five in the typical form of this species. The present study regards it to fall within the range of variation.

Occurrence. Rare in the Yonahama and Minebari formations.

\section{Genus PLANOGLABRATELA Seiglie and Bermúdez, 1965 \\ Planoglabratella opercularis (d'Orbigny)}

Figure 29.19

1839 Rosalina opercularis d'Orbigny (a), p. 93, pl. 3, figs. 24, 25.

1964 Neoconorbina opercularis (d'Orbigny) LeRoy, p. F36, pl. 9, figs. 9, 10.

1987 Planoglabratella opercularis (d'Orbigny) Loeblich and Tappan, pl. 621, figs. 21-23.

1994 Planoglabratella opercularis (d'Orbigny) Jones, p. 95, pl. 89, figs. 8, 9.

Occurrence. A single juvenile specimen from the Minebari Formation.

Superfamily SIPHONINOIDEA Cushman, 1927a

Family SIPHONINIDAE Cushman, 1927a

Subfamily SIPHONININAE Cushman, 1927a

Genus SIPHONINA Reuss, 1850

Siphonina australis Cushman

Figures 29.20, 29.21

1927 Siphonina australis Cushman (b), p. 8, pl. 2, fig. 6, pl. 3, fig. 7 .

1941 Siphonina australis Cushman - LeRoy (Part 1), p. 41, pl. 2, figs. 88-90; LeRoy (Part 2), p. 84, pl. 4, figs. 10-12.

1941 Siphonina australis Cushman - LeRoy (Part 2), p. 89, pl. 4, figs. 1-3.

1964 Siphonina bradyana Cushman - LeRoy, p. F39, pl. 4, figs. 5, 6 (non Siphonina bradyana Cushman, 1927b).

1988 Siphonina bradyana Cushman - Wang, Zhang, Zhao, Min, Bian, Zheng, Cheng, and Chen, p. 161, pl. 24, fig. 13; Marle, p. 149, pl. 2, fig. 13.

1994 Siphonina bradyana Cushman — Loeblich and Tappan, p. 143, pl. 298, figs. 1-9.

1998 Siphonina bradyana Cushman - Hess, p. 90 , pl. 14, figs. 5,6 .
Remarks. Siphonina bradyana Cushman (1927b) has a shorter neck and more fimbriate and broader keel than $S$. australis.

Occurrence. Rare in the Oura, Yonahama, and Minebari formations.

Superfamily DISCORBINELLOIDEA Sigal, 1952

Family PARRELLOIDIDAE Hofker, 1956a

Genus CIBICIDOIDES Thalmann, 1939 Cibicidoides ornatus (Cushman)

Figures 29.22-29.24

1921 Truncatulina ungeriana (d'Orbigny) var. ornata Cushman, p. 317, text-fig. 12.

1964 Cibicides ornatus (Cushman) - LeRoy, p. F45, pl. 8, figs. 19-21.

1994 Heterolepa ornatus (Cushman) - Loeblich and Tappan, p. 162, pl. 314, figs. 1-10.

Diagnosis. Moderate-sized species with nearequally biconvex, numerous chambers about 11-13 in final whorl, coarsely pitted surface.

Occurrence. Sporadic in the Onogoshi, Yonahama, and Minebari formations.

Cibicidoides pachyderma (Rzehak)

Figures 30.1-30.4

1886 Truncatulina pachyderma Rzehak, p. 87, pl. 1, fig. 5.

1964 Cibicides pseudoungerianus (Cushman) — LeRoy, p. F45, pl. 8, figs. 13-15 (?non Truncatulina pseudoungeriana Cushman, 1922c).

1986 Cibicidoides pachyderma (Rzehak) Morkhoven, Berggren, and Edwards, p. 68 , pl. 22, fig. 1.

1988 Cibicides pseudoungerianus (Cushman) - Wang, Zhang, Zhao, Min, Bian, Zheng, Cheng, and Chen, p. 162, pl. 25, figs. 15, 16.

1989 Cibicides pseudoungerianus (Cushman) — Ōki, p. 151, pl. 21, fig. 5.

1992 Cibicidoides pachyderma (Rzehak) Hatta and Ujiié (b), p. 187, pl. 35, fig. 5.

1994 Cibicidoides pachyderma (Rzehak) Jones, p. 98, pl. 94, fig. 9.

1995 Cibicidoides pachyderma (Rzehak) Ujiié, p. 67 , pl. 9, fig. 5 .

Remarks. This is one of the more familiar species in bathyal waters around southern Japanese Islands. It has bioconvex or umbilically more convex test, moderate test size for the genus, about nine to eleven chambers in final whorl, coarsely pitted spiral side, and moderately curved sutures. Hatta and Ujiié (1992b) suggested that it had been 
recorded previously as Cibicidoides pseudoungerianus (Cushman). Two aberrant forms that have inflated chambers are also illustrated here. Wang and Lutze (1986) noted several inflated varieties occasionally seen in calcareous hyaline taxa including Cibicidoides, and this is one of them.

Occurrence. Common in the Onogoshi, Yonahama, and Minebari formations.

\section{Cibicidoides sp. A \\ Figures 30.5, 30.6}

Description. Test very small for the genus, approximately $0.12-0.15 \mathrm{~mm}$ in diameter; trochospiral, biconvex but umbilical side more convex; periphery rounded; seven to eight chambers in the last whorl; sutures on dorsal side strongly curving backwards, umbilical side radiate; aperture interiomarginal extending to edge of periphery of last chamber; test thin and finely perforate.

Remarks. We could not find any taxon to assign this specimen to. This form might be a juvenile of any species of Cibicidoides, although all our specimens show the similar small test size and we regard them as adult.

Occurrence. Thirty-nine specimens from only a single sample of the Yonahama Formation.

Genus PARRELLOIDES Hofker, 1956a Parrelloides bradyi (Trauth)

Figures 30.7, 30.8

1918 Truncatulina bradyi Trauth, p. 235.

1951 Cibicides hyalina Hofker, p. 359, figs. 244, 245.

1964 Eponides hyalinus (Hofker) - LeRoy, p. F37, pl. 7, figs. 24-26.

1966 Parrelloides bradyi (Trauth) - Belford, pp. 100-102, pl. 11, figs. 10-19.

1968 Cibicidoides bradyi (Trauth) - Huang, p. 56, pl. 13, figs. 22, 23.

1988 Parrelloides bradyi (Trauth) - Marle, p. 148, pl. 3, figs. 16, 17.

1989 Cibicidoides bradyi (Trauth) - Hermelin, pp. 85,86 , pl. 17 , figs. $2-4$.

1990 Parrelloides bradyi (Trauth) - Akimoto, pp. 206, 207, pl. 20, fig. 2, pl. 23, fig. 8.

1992 Cibicidoides bradyi (Trauth) - Kaiho, pl. 6, figs. 3, 4, 6, 7 .

1994 Gyroidina bradyi (Trauth) - Jones, p. 99, pl. 95, fig. 5.

1994 Parrelloides bradyi (Trauth) - Loeblich and Tappan, p. 144, pl. 301, figs. 1-9.
1996 Parrelloides hyalinus (Hofker) - Revets (b), p. 68, pl. 3, figs. 9, 10.

2000 Cibicidoides bradyi (Trauth) —_ Ohkushi, Thomas, and Kawahata, p. 139, pl. 4, fig. 6.

Remarks. We regard Cibicides hyalina Hofker, type species of the genus Parrelloides, as a junior synonym of $P$. bradyi. Genus Parrelloides is distinguished from Cibicidoides in the smaller size and rounded periphery, and from Gyroidina in more convex spiral side, rounded periphery, and optically radial microstructure.

Occurrence. Common in the Yonahama Formation, rare in the Minebari Formation.

Family PSEUDOPARRELLIDAE Voloshinova in Voloshinova and Dain, 1952

Subfamily PSEUDOPARRELLINAE Voloshinova in Voloshinova and Dain, 1952

Genus ALABAMINOIDES Gudina and Saidova, 1967

Alabaminoides exiguus (Brady)

Figures 30.9, 30.10

1884 Pulvinulina exigua Brady, p. 696, pl. 103, figs. 13, 14.

1988 Epistominella exigua (Brady) - Marle, p. 143, pl. 3, figs. $6-8$.

1989 Epistominella exigua (Brady) - Inoue, pp. 153, 154, pl. 18, fig. 12, pl. 26, fig. 2.

1990 Pseudoparella exigua (Brady) - Akimoto, p. 208, pl. 20, fig. 7, pl. 24, fig. 3.

1990 Epistominella exigua (Brady) - Ujié, p. 32, pl. 14, fig. 1.

1992 Epistominella exigua (Brady) - Kaiho, pl. 8, fig. 6.

1994 Alabaminoides exiguus (Brady) - Jones, p. 103, pl. 103, figs. 13, 14.

1994 Pseudoparella exigua (Brady) - Loeblich and Tappan, p. 146, pl. 307, figs. 1-7; Akimoto, p. 285, pl. 3, fig. 8.

1994 Epistominella exigua (Brady) - Xu and Ujié, p. 518, figs.7-1, 7-2.

1995 Epistominella exigua (Brady) - Ujiié, pp. 67, 68, pl. 10, fig. 3.

1998 Epistominella exigua (Brady) - Hess, p. 80, pl. 14, figs. 15, 16.

2000 Epistominella exigua (Brady) - Ohkushi, Thomas, and Kawahata, p. 139, pl. 3, fig. 1. 


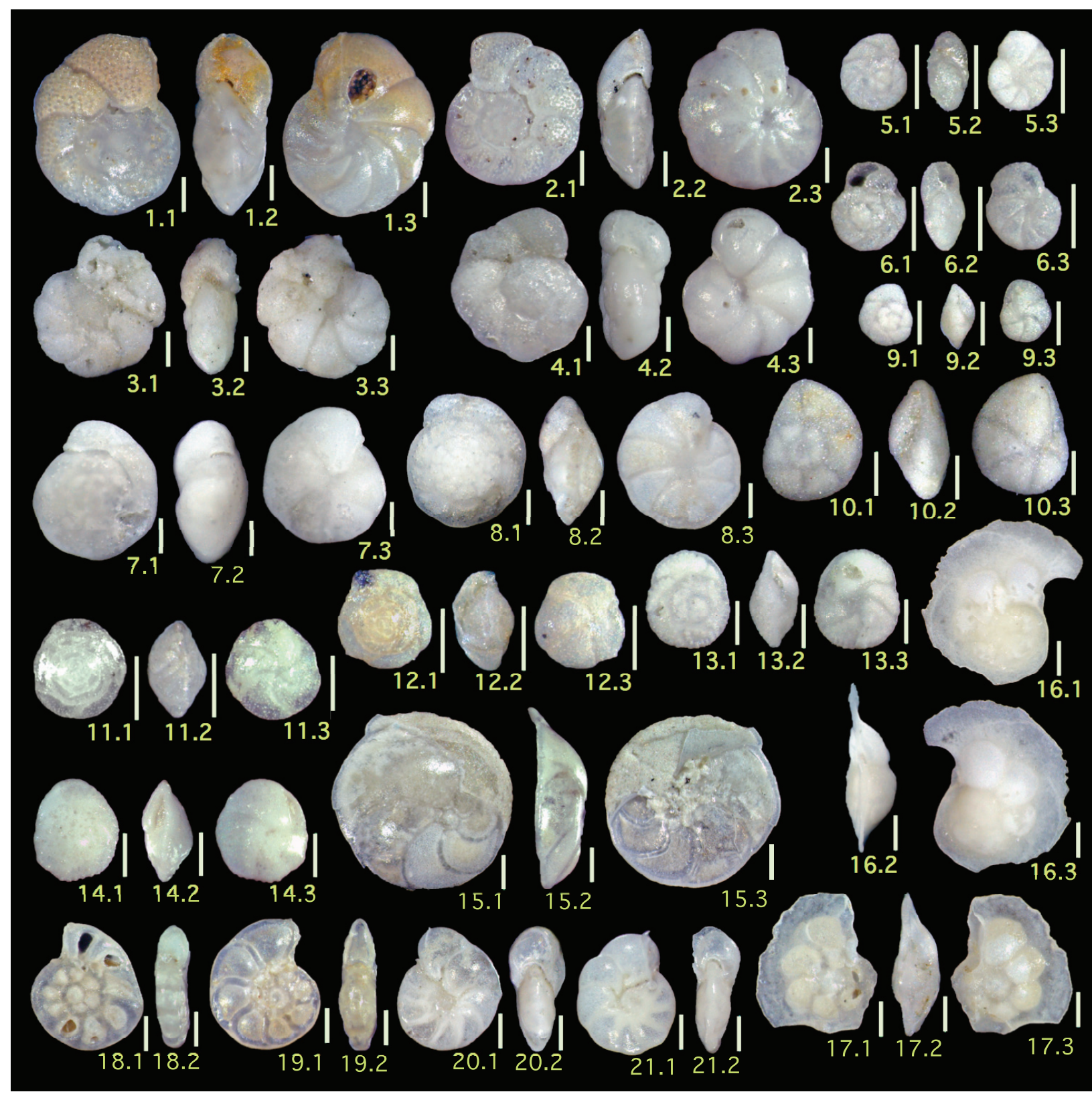

FIGURE 30. Family Parrelloididae, Pseudoparrellidae, Discorbinellidae, and Planulinidae. All scale bars $=0.1 \mathrm{~mm}$. 1-4. Cibicidoides pachyderma (Rzehak), 1: MPC-26203 from sample MK01 (Yonahama Formation, PL2); 2: MPC26204 from sample 4-2 (Minebari F., PL5); 3: MPC-26205 from sample 3-2-up-sand (Onogoshi F., PL1); 4: MPC26206 from sample 5-2 (Minebari F., PL5); 3 and 4, inflate-chamber variation. 5, 6. Cibicidoides sp.A, 5: MPC-26207, 6: MPC-26208, both from sample MK01 (Yonahama F., PL2). 7, 8. Parrelloides bradyi (Trauth), 7: MPC-26409 from sample 7 (Yonahama F., PL4); 8: MPC-26410 from sample MK01 (Yonahama F., PL2). 9, 10. Alabaminoides exiguus (Brady), 9: MPC-26108 from sample MK01 (Yonahama F., PL2); 10: MPC-26109 from sample MK02A (Minebari F., PL5). 11, 12. Eilohedra rotunda (Husezima and Maruhasi), 11: MPC-26230 from sample 1-2 (Minebari F., PL5); 12: MPC-26231 from sample MK02B (Minebari F., PL5). 13, 14. Facetocochlea pulchra (Cushman), 13: MPC-26255 from sample 3-2-low-silt (Onogoshi F., PL1); 14: MPC-26256 from sample 5-2 (Minebari F., PL5). 15. Discorbinella montereyensis Cushman and Martin, MPC-26223 from sample 12 (Minebari F., PL5). 16, 17. Laticarinina pauperata (Parker and Jones), 16: MPC-26348 from sample 7 (Yonahama F., PL4); 17: MPC-26349 from sample MK01 (Yonahama F., PL2). 18-21. Hyalinea balthica (Schröter), 18: MPC-26328 from sample MK01 (Yonahama F., PL2); 19: MPC-26329 from sample MK02B (Minebari F., PL5); 20: MPC-26330 from sample 5 (Minebari F., PL5); 21: MPC26331 from sample 5-2 (Minebari F., PL5); 20 and 21, inflated-chamber variation. 
2001 Epistominella exigua (Brady) - Hayward, Carter, Grenfell, and Hayward, figs.15-Y, $15-Z, 15-A A$.

Remarks. This species has been listed either as a member of the genus Pseudoparella or of Epistominella among Japanese workers who focused on the character of the aperture parallel to the periphery. We follow Jones's (1994) placement in the genus Alabaminoides. It is characterized by a noncarinate periphery, a small number of chambers and a slightly raised dorsal (biconvex) test, not like Epistominella.

Occurrence. Rare in the Yonahama Formation, sporadic in the Minebari Formation.

Genus EILOHEDRA Lipps, 1965

Eilohedra rotunda (Husezima and Maruhasi)

Figures $30.11,30.12$

1944 Eponides rotundus Husezima and Maruhasi, p. 399, pl. 34, fig. 12.

1958 Epistominella levicula Resig, p. 304, textfig. 16.

1967 Epistominella nipponica Kuwano, in Matoba, text-fig. 8, pl. 26, fig. 13.

1988 Epistominella naraensis (Kuwano) Wang, Zhang, Zhao, Min, Bian, Zheng, Cheng, and Chen, p. 156, pl. 22, fig. 13 (non Pseudoparrella naraensis Kuwano, 1950).

1989 Eilohedra levicula (Resig) - Ōki, pp. 126, 172, pl. 14, fig. 2.

1994 Epistominella levicula Resig - Xu and Ujiié, p. 518, fig. 7-3.

1995 Eilohedra levicula (Resig) - Ujiié, p. 67, pl. 10, fig. 2.

Remarks. This very small species has priority over $E$. levicula and E. nipponica. Type specimens of this species were lost in World War II; a neotype has not yet been designated. This is a familiar species in the Sea of Japan region, meaning that the selection of a neotype would not be difficult. It also differs from Pseudoparrella naraensis Kuwano in a strongly curved suture on the umbilical side.

Occurrence. Rare in the Yonahama Formation, common in the Minebari Formation.

Genus FACETOCOCHLEA Loeblich and Tappan, 1994

Facetocochlea pulchra (Cushman)

Figures 30.13, 30.14

1933 Pulvinulinella pulchra Cushman (b), p. 92, pl. 9, fig. 10.
1964 Neoconorbina pacifica LeRoy, p. F36, pl. 4, figs. 26-28.

1988 Epistominella pulchra (Cushman) Marle, p. 143, pl. 5, figs. 13-15.

1992 Epistominella pulchra (Cushman) - Hatta and Ujiié (b), pp. 187, 188, pl. 36, fig. 2.

1994 Facetocochlea pulchra (Cushman) - Loeblich and Tappan, p. 145, pl. 304, figs. 110.

Remarks. This species had been assigned to the genus Epistominella until Loeblich and Tappan (1994) erected Facetocochlea based on the presence of rows of large pores on the spiral side of the test.

Occurrence. Rare in the Onogoshi and Minebari formations.

Family DISCORBINELLIDAE Sigal, 1952

Subfamily DISCORBINELLINAE Sigal, 1952

Genus DISCORBINELLA Cushman and Martin, 1935

Discorbinella montereyensis Cushman and Martin Figure 30.15

1935 Discorbinella montereyensis Cushman and Martin, p. 89, pl. 14, fig. 13.

1987 Discorbinella montereyensis Cushman and Martin - Loeblich and Tappan, pl. 63, figs. $1-3$.

1994 Discorbinella montereyensis Cushman and Martin - Loeblich and Tappan, p. 148 , pl. 113 , figs. $1-6$.

Occurrence. A single specimen from the Minebari Formation.

Genus LATICARININA Galloway and Wissler, 1927b

Laticarinina pauperata (Parker and Jones)

Figures $30.16,30.17$

1865 Pulvinulina repanda (Fichtel and Moll) var. menardii subvar. pauperata Parker and Jones, p. 395, pl. 16, figs. 50, 51.

1941 Laticarinina pauperata (Parker and Jones) - LeRoy (Part 1), p. 46, pl. 2, figs. 18, 19; LeRoy (Part 2), p. 88, pl. 6, figs. 30, 31.

1964 Laticarinina pauperata (Parker and Jones) — LeRoy, p. F44, pl. 9, fig. 25.

1965 Laticarinina pauperata (Parker and Jones) - Todd, p. 51, pl. 21, fig. 6.

1966 Laticarinina pauperata (Parker and Jones) - Belford, pp. 92, 93, pl. 14, figs. 9-13.

1968 Laticarinina pauperata (Parker and Jones) — Huang, p. 57, pl. 13, figs. 34, 39. 
1987 Laticarinina pauperata (Parker and Jones)

- Loeblich and Tappan, pl. 631, figs. 14.

1988 Laticarinina pauperata (Parker and Jones)

- Marle, p. 145, pl. 3, figs. 1-3.

1990 Laticarinina pauperata (Parker and Jones) — Ujiié, p. 33, pl. 14, figs. 3, 4.

1994 Laticarinina pauperata (Parker and Jones) - Jones, p. 104, pl. 104, figs. 1-3; Loeblich and Tappan, p. 148, pl. 312, figs. 15.

1998 Laticarinina pauperata (Parker and Jones) - Hess, pp. 83, 84, pl. 9, fig. 13.

Occurrence. Sporadic in the Yonahama Formation.

Superfamily PLANOBULIMINOIDEA Schwager, 1877

Family PLANULINIDAE Bermúdez, 1952

Genus HYALINELA Hofker, 1951

Hyalinea balthica (Schröter)

Figures 30.18-30.21

1782 'Ammonshörner der Ostsee' Schröter, p. 120.

1783 'Das platte Ammonshorn aus der Ostsee' Schröter, p. 20, pl. 1, fig. 2.

1791 Nautilus balthicus Gmelin, p. 3370.

1941 Operculina ammonoides (Gronovius) LeRoy (Part 2), p. 78, pl. 6, figs. 24, 25 (non Nautilus ammonoides Gronovius, 1781).

1964 Hyalinea balthica (Schröter) - LeRoy, p. F44, pl. 9, figs. 34-36.

1966 Hyalinea balthica (Gmelin) - Belford, pp. 124,125 , pl. 14 , figs. $1-8$, text-figs. 131-13-3.

1977 Hyalinea florenceae McCulloch, p. 452, pl. 181, fig. 1.

1986 Hyalinea balthica (Schroeter) - Wang and Lutze, p. 57, pl. 3, figs. 1, 11, 12,

1987 Hyalinea balthica (Schröter) - Loeblich and Tappan, pl. 632, figs. 5-8.

1988 Hyalinea balthica (Schroeter) - Wang, Zhang, Zhao, Min, Bian, Zheng, Cheng, and Chen, p. 164, pl. 26, figs. 4, 5; Marle, p. 145, pl. 2, fig. 21.

1989 Hyalinea balthica (Schröter) - Ōki, p. 139, pl. 17, fig. 6.

1989 Hyalinea balthica (Schroeter) - Inoue, pl. 28, fig. 9, pl. 31, fig. 12.
1990 Hyalinea balthica (Schröter) - Akimoto, p. 202, pl. 20, fig. 5.

1994 Hyalinea balthica (Schroeter) - Jones, $\mathrm{p}$. 110, pl. 112, figs. 1, 2.

1994 Hyalinea florenceae McCulloch - Loeblich and Tappan, pp. 148, 149, pl. 313, figs. $1-10$.

1995 Hyalinea balthica (Schröter) - Ujiié, p. 68, pl. 10, figs. 4, 5.

2001 Hyalinea balthica (Schröter) - Kawagata, pp. 98, 99, fig. 11-8.

2002 Hyalinea balthica (Schröter) - Akimoto, Matsui, Shimokawa, and Furukawa, p. 20, pl. 52, fig. 1.

Remarks. Gmelin (1791) erected this species on the basis of a description by Schröter $(1782,1783)$ (see also Belford, 1966). Loeblich and Tappan (1994) regarded Hyalinea florenceae McCulloch as a distinct species, but we regard it here as a variety of $H$. balthica with inflated chambers, following the study by Wang and Lutze (1986). In specimens of inflated variation, limbation of sutures that characterize typical $H$. balthica is no longer visible from the outside.

Hyalinea inflata Ujiié and Kusukawa (1969, p. 767 , pl. 2, figs. 1-3) has inflated chambers similar to $H$. florenceae, but chamber inflation begins from a younger stage as based on a figure in Oki (1989, pl. 18, fig. 1); we regard it as a distinct species.

Occurrence. Common in all the formations of the present study. Specimens of inflated variation are sporadic in the Onogoshi and Minebari formations.

Genus PLANULINA d'Orbigny, 1826

Planulina retia Belford

Figures 31.1, 31.2

1966 Planulina retia Belford, p. 122, pl. 11, figs. $1-9$.

1994 Planulina retia Belford - Loeblich and Tappan, p. 149, pl. 315, figs. $1-11$, pl. 316, figs. $4-7$.

Diagnosis. Large- to moderate-sized species with compressed plano-convex test, moderately pitted surface and evolute umbilical coiling.

Occurrence. Common in the Minebari Formation. Family CIBICIDIDAE Cushman, 1927a

Subfamily CIBICIDINAE Cushman, 1927a Genus CIBICIDES Montfort, 1808 Cibicides refulgens de Montfort

Figures 31.3, 31.4

1808 Cibicides refulgens de Montfort, p. 123. 
1921 Truncatulina refulgens (de Montfort) Cushman, p. 312, pl. 63, fig. 1.

1964 Cibicides refulgens de Montfort - LeRoy, p. F44, pl. 8, figs. 22-24.

1966 Cibicides sp. cf. C. refulgens Montfort Belford, p. 133, pl. 23, figs. 1-6, text-figs. 15-3-15-10.

1987 Cibicides refulgens de Montfort — Loeblich and Tappan, pl. 634, figs. 1-3.

1989 Cibicides cf. refulgens de Montfort Inoue, pl. 31, fig. 4.

1990 Cibicides refulgens de Montfort Akimoto, p. 196, pl. 23, fig. 5.

1994 Cibicides refulgens de Montfort - Jones, p. 97, pl. 92, figs. 7-9; Loeblich and Tappan, p. 149, pl. 318, figs. 7-9.

1995 Cibicides refulgens de Montfort - Ujiié, p. 68 , pl. 11, fig. 1.

Occurrence. Rare in the Yonahama Formation, sporadic in the Minebari Formation.

\section{Cibicides tenuimargo (Brady)} Figure 31.5

1884 Truncatulina tenuimargo Brady, p. 62, pl. 93, fig. 3.

1964 Cibicides tenuimargo (Brady) - LeRoy, p. F44, pl. 8, figs. 30-32.

1966 Cibicides tenuimargo (Brady) - Belford, $p$. 135, pl. 23, figs. $7-9$, text-figs. $15-11-15-$ 13.

1990 Cibicides tenuimargo (Brady) - Akimoto, p. 195, pl. 23, fig. 6.

1994 Parbicarinina tenuimargo (Brady) Jones, p. 97, pl. 93, fig. 3.

1994 Cibicides tenuimargo (Brady) - Loeblich and Tappan, p. 149, pl. 316, figs. $1-3$, pl. 317, figs. $1-10$.

Occurrence. Two specimens from a single sample of the Minebari Formation.

Genus FONTBOTIA González-Donoso and Linares, 1970

Fontbotia wuellerstorfi (Schwager)

Figures 31.6, 31.7

1866 Anomalina wuellerstorfi Schwager, pp. 258, 259, pl. 7, fig. 105.

1921 Truncatulina wuellerstorfi (Schwager) Cushman, pp. 314, 315, pl. 64, fig. 1.

1941 Cibicides wuellerstorfi (Schwager) LeRoy (Part 1), p. 46, pl. 1, figs. 27-29; LeRoy (Part 2), p. 89, pl. 3, figs. 7-9.
1944 Cibicides wuellerstorfi (Schwager) LeRoy (Part 2), p. 93, pl. 6, figs. 13-15.

1964 Cibicides wuellerstorfi (Schwager) LeRoy, p. F45, pl. 8, figs. 25, 26.

1965 Planulina wuellerstorfi (Schwager) Todd, pp. 51, 52, pl. 23, figs. 3-5.

1966 Planulina wuellerstorfi (Schwager) - Belford, pp. 120, 121, pl. 10, figs. 1-6.

1968 Cibicidoides wuellerstorfi (Schwager) Huang, p. 56, pl. 13, fig. 26.

1987 Fontbotia wuellerstorfi (Schwager) - Loeblich and Tappan, pl. 634, figs. 10-12, pl. 635, figs. 1-3.

1988 Planulina wuellerstorfi (Schwager) Marle, p. 148, pl. 3, figs. 16, 17.

1989 Planulina wuellerstorfi (Schwager) Inoue, p. 155, pl. 27, fig. 4, pl. 28, fig. 13.

1990 Fontbotia wuellerstorfi (Schwager) - Ujiié, pp. 35,36 , pl. 17 , figs. $1-5$.

1990 Cibicides wuellerstorfi (Schwager) Akimoto, p. 195, pl. 23, fig. 7.

1994 Cibicidoides wuellerstorfi (Schwager) Jones, p. 98, pl. 93, figs. 8, 9.

1994 Fontbotia wuellerstorfi (Schwager) - Loeblich and Tappan, p. 150, pl. 319, figs. 712.

1994 Cibicides wuellerstorfi (Schwager) Akimoto, p. 284, pl. 3, fig. 10.

1994 Planulina wuellerstorfi (Schwager) - Xu and Ujié, p. 518, figs. 7-9, 7-10.

1998 Cibicidoides wuellerstorfi (Schwager) Hess, pp. 78, 79, pl. 16, figs. 5-7.

2000 Planulina wuellerstorfi (Schwager) Ohkushi, Thomas, and Kawahata, p. 144, pl. 4, fig. 1.

Remarks. This is a quite familiar bathyal species, although its generic position varies among researchers. Schweizer et al. (2009) discussed the taxonomy of Cibicides, Cibicidoides, and other genera including "Cibicidoides wuellerstorfi" based on morphology and rDNA sequence. Their results indicate that this species is not phylogenetically close to species of Cibicides but to Cibicidoides, which implies that "wuellerstorfi" should be assigned to Cibicidoides or be separated as Fontbotia following Loeblich and Tappan (1987, 1994).

Occurrence. Common in the Onogoshi, Yonahama, and Minebari formations. 


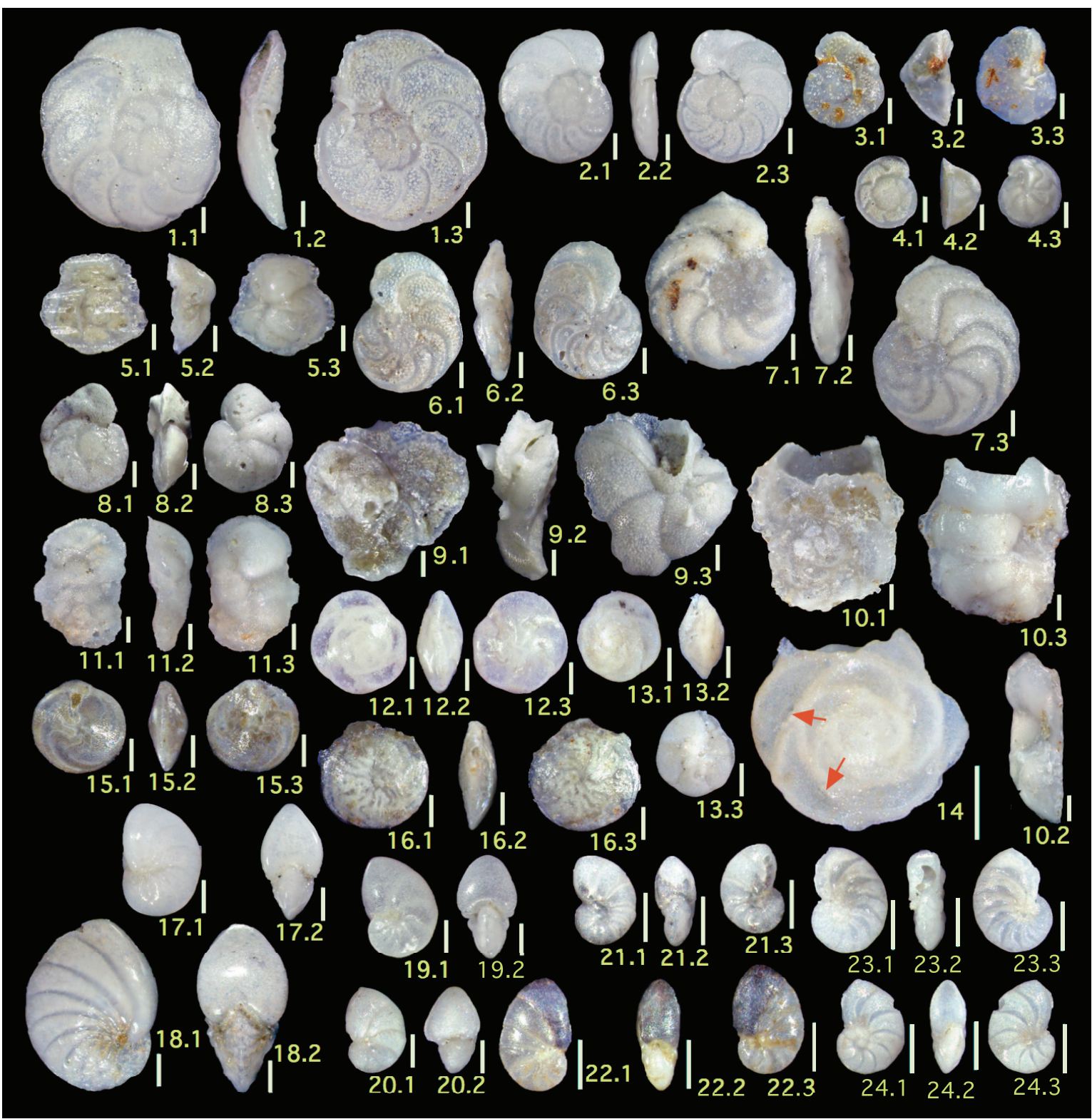

FIGURE 31. Family Planulinidae, Cibicididae, Epistomariidae, Amphisteginidae, and Nonionidae. All scale bars = $0.1 \mathrm{~mm}$. 1, 2. Planulina retia Belford, 1: MPC-26416 from sample 2 (Minebari Formation, PL5); 2: MPC-26417 from sample 5-2 (Minebari F., PL5). 3, 4. Cibicides refulgens de Montfort, 3: MPC-26197 from sample 2-2 (Minebari F., PL5); 4: MPC-26198 from sample 14-2 (Yonahama F., PL4). 5. Cibicides tenuimargo (Brady), MPC-26199 from sample 4-2 (Minebari F., PL5); stripe pattern on the dorsal side implies attached material. 6, 7. Fontbotia wuellerstorfi (Schwager), 6: MPC-26272 from sample MK01 (Yonahama F., PL2); 7: MPC-26273 from sample MK02A (Minebari F., PL5). 8, 9. Lobatula lobatula (Walker and Jacob), 8: MPC-26367 from sample 5 (Minebari F., PL5); 9: MPC-26368 from sample 5-2 (Minebari F., PL5). 10, 11. Dyocibicides biserialis Cushman and Valentine, 10: MPC-26224 from sample 2-2 (Minebari F., PL5); 11: MPC-26225 from sample 3-2-up-sand (Onogoshi F., PL1). 12-14. Pseudoeponides japonicus Uchio, 12: MPC-26430 from sample 14 (Yonahama F., PL4); 13: MPC-26431 and 14: MPC-26548 from sample MK02B (Minebari F., PL5); arrows in 14 shows slit opening along dorsal sutures. 15, 16. Amphistegina lessonii d'Orbigny, 15: MPC-26123 from sample 2 (Minebari F., PL5); 16: MPC-26124 from sample 2-2 (Minebari F., PL5); all specimens of this study are juveniles. 17, 18. Nonion japonicum Asano, 17: MPC-26392 from sample 5-2 (Minebari F., PL5); 18: MPC-26393 from sample 11 (Onogoshi F., ?PL1). 19, 20. Nonionellina labradorica (Dawson), 19: MPC-26394 from sample 1-2 (Minebari F., PL5); 20: MPC-26395 from sample MK02B (Minebari F., PL5). $21,22$. Pseudononion cuevasensis Saunder and Müller-Merz, 21: MPC-26438 from sample 10 (Onogoshi F., PL2); 22: MPC-26439 from sample MK02B (Minebari F., PL5). 23, 24. Pseudononion subcostatum Fujita and Ito, 23: MPC26440 from sample 14-2 (Yonahama F., PL4); 24: MPC-26441 from sample MK02B (Minebari F., PL5). 
Genus LOBATULA Fleming, 1828

Lobatula lobatula (Walker and Jacob)

Figures 31.8, 31.9

1798 Nautilus lobatulus Walker and Jacob in Kanmacher, p. 642, pl. 14, fig. 36.

1921 Truncatulina lobatula (Walker and Jacob) - Cushman, p. 313, pl. 63, fig. 2.

1941 Cibicides lobatulus (Walker and Jacob) LeRoy (Part 3), p. 119, pl. 1, figs. 12-14.

1959 Cibicides lobatulus (Walker and Jacob) Graham and Militante, pp. 116, 117, pl. 19, fig. 12.

1964 Cibicides lobatulus (Walker and Jacob) LeRoy, p. F45, pl. 8, figs. 10-12.

1984 Cibicides lobatulus (Walker and Jacob) Rögl and Hansen, p. 68, pl. 26, figs. 9-11.

1985 Cibicides lobatulus (Walker and Jacob) Papp and Schmid, p. 64, pl. 56, figs. 1-5, p. 65 , pl. 57 , figs. $1-3$.

1987 Lobatula lobatula (Walker and Jacob) Loeblich and Tappan, pl. 637, figs. 10-13.

1988 Cibicides lobatulus (Walker and Jacob) Wang, Zhang, Zhao, Min, Bian, Zheng, Cheng, and Chen, p. 162, pl. 25, figs. 1214; Marle, p. 141, pl. 4, figs. 18, 19.

1992 Cibicides lobatulus (Walker and Jacob) Hatta and Ujiié (b), pp. 188, 189, pl. 37, figs. 4,5 .

1994 Cibicides lobatulus (Walker and Jacob) Jones, p. 97, pl. 92, fig. 10, pl. 93, figs. 1, 4, 5, pl. 115, figs. 4, 5 .

1994 Lobatula lobatula (Walker and Jacob) Loeblich and Tappan, p. 150, pl. 316, figs. $1-7$.

2001 Cibicides lobatulus (Walker and Jacob) Kawagata, p. 99, fig. 12-1.

2002 Lobatula lobatulus (Walker and Jacob) Akimoto, Matsui, Shimokawa, and Furukawa, p. 20, pl. 54, fig. 1.

Remarks. Molecular phylogenetic study by Schweizer et al. (2009) has indicated that this species is very close to the genus Cibicidoides, not to Cibicides. Therefore, we distinguish Lobatula from Cibicidoides following Loeblich and Tappan (1987, 1994).

Occurrence. Sporadic in the Minebari Formation.

Subfamily STICHOCIBICIDINAE Saidova, 1981 Genus DYOCIBICIDES Cushman and Valentine, 1930
Dyocibicides biserialis Cushman and Valentine Figures 31.10, 31.11

1930 Dyocibicides biserialis Cushman and Valentine, p. 31, pl. 10, figs. 1, 2.

1977 Dyocibicides cf. biserialis Cushman and Valentine - McCulloch, pp. 464, 465, pl. 194, fig. 5, pl. 195, figs. 1, 3, 4-10, 14, 15 , pl. 196, figs. 1,2 .

1987 Dyocibicides biserialis Cushman and Valentine - Loeblich and Tappan, pl. 639, figs. 8-10.

1994 Dyocibicides biserialis Cushman and Valentine - Jones, p. 97, pl. 93, fig. 6.

2002 Dyocibicides biserialis Cushman and Valentine - Akimoto, Matsui, Shimokawa, and Furukawa, p. 21, pl. 54, fig. 2.

Occurrence. Sporadic in the Onogoshi and Minebari formations.

Superfamily ASTERIGERINOIDEA d'Orbigny, $1839 a$

Family EPISTOMARIIDAE Hofker, 1954

Subfamily EPISTOMARIINAE Hofker, 1954

Genus PSEUDOEPOIDES Uchio in Kawai, Uchio, Ueno, and Hozuki, 1950

Pseudoeponides japonicus Uchio

Figures 31.12-31.14

1950 Pseudoeponides japonica Uchio in Kawai, Uchio, Ueno, and Hozuki, p. 190, fig. 16.

1951 Pseudoeponides japonica Uchio - Uchio, pp. 38, 39, pl. 3, fig. 1.

1963 Pseudoeponides japonica Uchio - Matsunaga, pl. 45, fig. 7.

1950 Epistomaria miurensis Kuwano, pp. 315, 316, figs. 3, 10.

1951 Pseudoeponides japonicus Uchio Uchio, pp. 38, 39, pl. 3, fig. 1; Asano (part 14), p. 19, text-figs. $138,140$.

1964 Pseudoeponides japonicus Uchio LeRoy, p. F39, pl. 9, figs. 20-22.

1967 Pseudoeponides japonicus Uchio Matoba, pl. 26, fig. 20.

1994 Pseudoeponides japonicus Uchio - Loeblich and Tappan, p. 156, pl. 338, figs. 112.

2000 Epistomaria miurensis Kuwano - Scott, Takayanagi, Hasegawa, and Saito, p. 21, figs. 7.137-7.139.

2001 Pseudoeponides japonicus Uchio Kawagata, p. 101, fig. 12-4. 
Remarks. This is one of the commoner species in the Pliocene of Japan. Its taxonomic status once was in confusion since Kuwano (1950) described E. miurensis in the same year as Uchio erected $P$. japonicus. The date of issue of Uchio's paper is June 30th, 1950, that of Kuwano's is September 20th, 1950. These dates appear on the back cover print of each journal. However, what is confusing is that both journals indicate the month of publication to be "June 1950" on the front page. Consequently, the issue date is the single key to decide priority based on the date of publication, even though the description by Uchio (1950) is incomplete in lacking description of type horizon, decision, and repository of type specimens. Kuwano (1950) did provide a systematic description and a fine drawing of specimens. Uchio published an additional description of $P$. japonicus in 1951.

Occurrence. Common in all the formations of the present study.

Family AMPHISTEGINIDAE Cushman, 1927a

Genus AMPHISTEGINA d'Orbigny, 1826

Amphistegina lessonii d'Orbigny

Figures 31.15, 31.16

1826 Amphistegina lessonii d'Orbigny, p. 304, pl. 17, figs. 1-4.

1865 Amphistegina lessonii d'Orbigny - Parker, Jones, and Brady, p. 34, pl. 3, figs. 91, 92.

1941 Amphistegina lessonii d'Orbigny — LeRoy (Part 1), p. 41, pl. 3, figs. 18, 19.

1977 Amphistegina lessonii d'Orbigny Larsen, pp. 274, 275, pl. 1, figs. 1-9.

1987 Amphistegina lessonii d'Orbigny - Loeblich and Tappan, pl. 677, figs. 3-5.

1988 Amphistegina lessonii d'Orbigny - Marle, p. 139, pl. 1, fig. 5.

1992 Amphistegina lessonii d'Orbigny - Hatta and Ujiié (b), p. 195, pl. 42, fig. 4.

1994 Amphistegina lessonii sensu Parker, Jones, and Brady - Jones, pp. 109, 110, pl. 111, figs. 2, 4-7.

1994 Amphistegina lessonii d'Orbigny - Loeblich and Tappan, pp. 156, 157, pl. 340, figs. 1-9.

1995 Amphistegina lessonii d'Orbigny - Ujiié, pp. 68,69 , pl. 11 , fig. 3 .

Remarks. This large form is a typical shallowwater species. The present specimens are small, up to $0.34 \mathrm{~mm}$ in diameter, and have probably been transported by gravity currents from shallower areas.
Occurrence. A single specimen each from two samples of the Minebari Formation.

Superfamily NONIONOIDEA Schultze, 1854

Family NONIONIDAE Schultze, 1854

Subfamily NONIONINAE Schultze, 1854

Genus NONION Montfort, 1808

Nonion japonicum Asano

Figures 31.17, 31.18

1938 Nonion japonicum Asano (b), p. 593, pl. 15, figs. $1,2$.

1959 Nonion japonicum Asano - Graham and Militante, pp. 71, 72, pl. 11, fig. 1.

1964 Nonion japonicum Asano - LeRoy, p. F27, pl. 10, figs. 12, 13.

1989 Florilus japonicus (Asano) - Ōki, pp. 146, 174, pl. 20, fig. 1.

1994 Nonion subturgidum (Cushman) - Loeblich and Tappan, p. 158, pl. 343, figs. 19 (non Nonionina subturgida Cushman, 1924).

2002 Nonion japonicum Asano - Akimoto, Matsui, Shimokawa, and Furukawa, p. 24, pl. 59, fig. 3.

2006 Pseudononion japonicum Asano Takata, Irizuki, and Ishida, pl. 2, fig. 3 (non Pseudononion japonicum Asano, 1936a).

Remarks. This species is distinguished from $N$. subturgidum by having numerous chambers and granules in the umbilicus, as described by Asano (1938b).

Occurrence. Common in the Oura, Onogoshi, and Minebari formations.

Genus NONIONELLINA Voloshinova, 1958 Nonionellina labradorica (Dawson)

Figures 31.19, 31.20

1860 Nonionina labradorica Dawson, pp. 191, 192, text-fig. 4.

1938 Nonion scaphum Fichtel and Moll Asano (b), pp. 593, 594, pl. 15, fig. 4 (non Nautilus scapha Fichtel and Moll, 1798).

1982 Nonionellina labradorica (Dawson) Saunders and Müller-Merz, pp. 272, 273, pl. 2, figs. 14-18.

1987 Nonionellina labradorica (Dawson) - Loeblich and Tappan, pl. 689, figs. 8-17.

1988 Nonion scaphum (Fichtel and Moll) Marle, p. 147, pl. 4, fig. 8.

1989 Nonionellina labradorica (Dawson) Inoue, pp. 157, 158, pl. 24, fig. 3, pl. 32, fig. 13 , pl. 33 , fig. 3 . 
Remarks. Asano (1938b) assigned this species to Nautilus scapha Fichtel and Moll, and his identification was followed in subsequent Japanese works. Nonion scaphum is a senior synonym of Nonionina communis d'Orbigny (1846) as demonstrated by Rögl and Hansen (1984). It is distinguished from $N$. labradorica in a lesser growth rate and more compressed test.

Occurrence. Sporadic in the Minebari Formation.

Genus PSEUDONONION Asano, 1936a

Pseudononion cuevasensis Sauder and MüllerMerz

Figures 31.21, 31.22

1982

Pseudononion cuevasensis Saunders and Müller-Merz, pp. 267, 268, pl. 4, figs. 3438.

2006 Pseudononion sp. A - Takata, Irizuki, and Ishida, pl. 2, fig. 4.

Diagnosis. A small species with bilaterally compressed test, inflated chambers, and depressed sutures.

Remarks. This species resembles Nonionina grateloupi d'Orbigny, but is differentiatied in having more inflated chambers and a thicker test.

Occurrence. Sporadic in the Onogoshi, Yonahama, and Minebari formations.

Pseudononion subcostatum Fujita and Ito

Figures 31.23, 31.24

1957 Pseudononion subcostatum Fujita and Ito, p. 510, pl. 10, fig. 1.

Diagnosis. A small species with limbate and curving sutures on both sides of test.

Remarks. This species was originally described from the Miocene of Fukushima, northern Japan. Our material exhibits a slightly more depressed shape, but this is here regarded as variation.

Occurrence. Rare in the Yonahama and Minebari formations.

Subfamily ASTRONONIONINAE Saidova, 1981

Genus ASTRONONION Cushman and Edwards, 1937

Astrononion hayamaense Kurihara

Figure 32.1

1971 Astrononion hayamaense Kurihara, p. 140, pl. 15 , fig. 8.

Remarks. This species was originally described from the Miocene of central Honshu, Japan. It is characterized by a small size and oval cross section.

Occurrence. A single specimen from the Oura Formation.
Astrononion stelligerum (d'Orbigny)

Figure 32.2

1839 Nonionina stelligera d'Orbigny (c), p. 128, pl. 3, figs. 1, 2.

1987 Astrononion stelligerum (d'Orbigny) Loeblich and Tappan, pl. 694, figs. 1, 2, 20 , 21.

1989 Astrononion stelligerum (d'Orbigny) — Ōki, pp. 145, 146, pl. 19, fig. 7.

1990 Astrononion stelligerum (d'Orbigny) Akimoto, p. 192, pl. 15, fig. 8.

1991 Astrononion stelligerum (d'Orbigny) Hayward, Carter, Grenfell, and Hayward, fig. 15- H, 15-I.

1994 Astrononion stelligerum (d'Orbigny) Jones, p. 107, pl. 109, figs. 3, 4; Loeblich and Tappan, pp. 158, 159, pl. 344, figs. 11-14.

2001 Astrononion stelligerum (d'Orbigny) Hayward, Carter, Grenfell, and Hayward, figs. 15-H, 15-I.

2002 Astrononion stelligerum (d'Orbigny) Akimoto, Matsui, Shimokawa, and Furukawa, p. 24, pl. 61, fig. 4.

Occurrence. A single juvenile specimen from the Yonahama Formation.

Genus FIJINONION Hornibrook, 1964

Fijinonion fijiensis (Cushman and Edwards)

Figures 32.3, 32.4

1937 Astrononion fijiensis Cushman and Edwards, p. 35, pl. 3, figs. 15, 16.

1987 Fijinonion fijiense (Cushman and Edwards) - Loeblich and Tappan, pl. 694, figs. 1510.

1994 Fijinonion fijiense (Cushman and Edwards) - Jones, p. 107, pl. 109, figs. 1, 2; Loeblich and Tappan, p. 159, pl. 346, figs. 14.

Occurrence. Three specimens from a single sample of the Minebari Formation.

Genus LAMINONONION Hornibrook, 1964 Laminononion tumidum (Cushman and Edwards) Figure 32.5

1937 Astrononion tumidum Cushman and Edwards, p. 33, pl. 3, fig. 17.

1987 Laminononion tumidum (Cushman and Edwards) - Loeblich and Tappan, pl. 694, figs. $16-19$. 


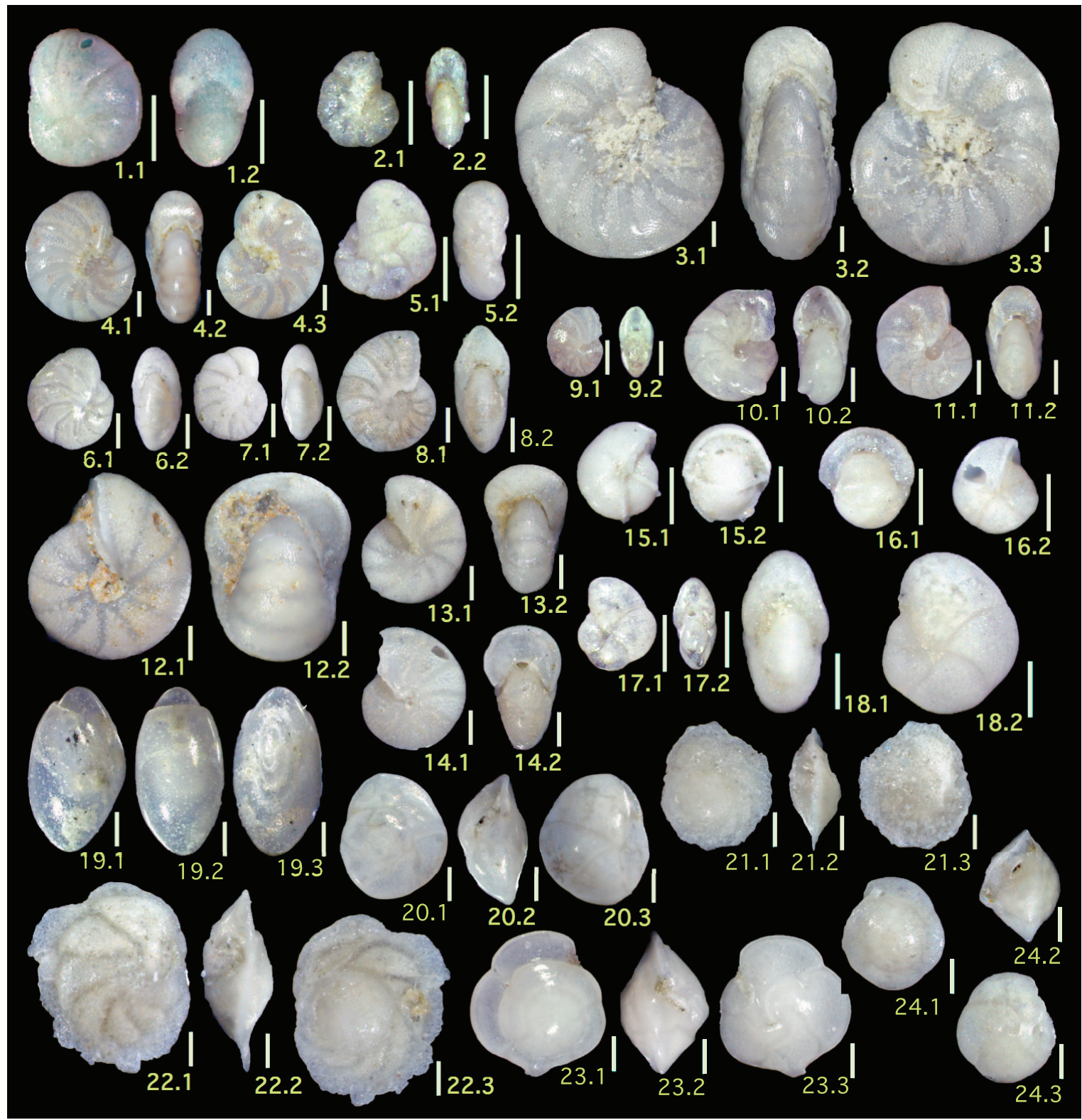

FIGURE 32. Family Nonionidae, Chilostomellidae, Alabaminidae, Osangulariidae, and Oridorsalidae. All scale bars = $0.1 \mathrm{~mm}$. 1. Astrononion hayamaense Kurihara, MPC-26136 from sample 18 (Oura Formation, ?PL1). 2. Astrononion stelligerum (d'Orbigny), MPC-26137 from sample MK01 (Yonahama F., PL2). 3, 4. Fijinonion fijiensis (Cushman and Edwards), 3: MPC-26259, 4: MPC-26260, both from sample 2-2 (Minebari F., PL5). 5. Laminononion tumidum (Cushman and Edwards), MPC-26345 from sample 15 (Yonahama F., PL3). 6, 7. Pacinonion umbilicatulum (Uchio), 6: MPC-26401 from sample 1 (Minebari F., PL5); 7: MPC-26402 from sample 12 (Minebari F., PL5). 8, 9. Melonis barleeanum (Williamson), 8: MPC-26370 from sample MK01 (Yonahama F., PL2); 9: MPC-26371 from sample MK02 (Minebari F., PL5). 10, 11. Melonis pacificum (Cushman), 10: MPC-26372 from sample 5-2 (Minebari F., PL5); 11: MPC26373 from sample MK02B (Minebari F., PL5). 12-14. Melonis pompilioides (Fichtel and Moll), 12: MPC-26374 from sample 3 (Onogoshi F., PL1); 13: MPC-26375 from sample 3-2-up-sand (Onogoshi F., PL1); 14: MPC-26376 from sample MK01 (Yonahama F., PL2). 15, 16. Pullenia bulloides (d'Orbigny), 15: MPC-26443 from sample 6 (Yonahama F., PL2); 16: MPC-26442 from sample MK01 (Yonahama F., PL2). 17, 18. Pullenia quinqueloba (Reuss), 17: MPC26444 from sample 1 (Minebari F., PL5); 18: MPC-26445 from sample 7 (Yonahama F., PL4). 19. Chilostomella cushmani Chapman, MPC-26194 from sample 5 (Minebari F., PL5). 20. Alabamina japonica (Asano), MPC-26107 from sample 12 (Minebari F., PL5). 21, 22. Osangularia culter (Parker and Jones), 21: MPC-26399 from sample 7 (Yonahama F., PL4); 22: MPC-26400 from sample 16 (Yonahama F., PL3). 23, 24. Oridorsalis umbonatus (Reuss), 23: MPC-26396 from sample 1-2 (Minebari F., PL5); 24: MPC-26397 from sample MK01 (Yonahama F., PL2). 
1994 Laminononion tumidum (Cushman and Edwards) - Loeblich and Tappan, p. 159, pl. 346, figs. 5-9.

2001 Laminononion tumidulum (Cushman and Edwards) - Kawagata, p. 103, fig. 13-5.

Occurrence. A single specimen from the Yonahama Formation.

Genus PACINONION Vella, 1962

Pacinonion umbilicatulum (Uchio)

Figures 32.6, 32.7

1952 Astrononion umbilicatulum Uchio, p. 36, pl. 32, text-fig. 1.

1963 Astrononion hanyudaense Matsunaga, pp. 108, 109, pl. 35, fig. 8.

1989 Astrononion umbilicatulum Uchio - Inoue, p. 157, pl. 32, fig. 18.

Occurrence. Rare in the Minebari Formation.

Subfamily PULLENIINAE Schwager, 1877

Genus MELONIS de Montfort, 1808

Melonis barleeanum (Williamson)

Figures 32.8, 32.9

1858 Nonionina barleeana Williamson, p. 32, pl. 3, figs. 68, 69 .

1960 Nonion parkerae Uchio, p. 60, pl. 4, figs. 9, 10.

1977 Melonis pacificaformis McCulloch, p. 438, pl. 180, fig. 3.

1987 Melonis barleeanum (Williamson) - Loeblich and Tappan, pl. 696, figs. 5, 6.

1988 Melonis barleeanum (Williamson) Wang, Zhang, Zhao, Min, Bian, Zheng, Cheng, and Chen, pp. 179, 180, pl. 32, fig. 14.

1989 Melonis parkerae (Uchio) - Inoue, pp. 158, 159, pl. 27, fig. 2, pl. 32, fig. 19.

1990 Melonis barleeanum (Williamson) - Ujiié, p. 52, pl. 29, fig. 4 .

1992 Melonis barleeanus (Williamson) - Kaiho, pl. 7, fig. 10; Kaiho and Nishimura, pl. 4, fig. 18.

1994 Melonis barleeanum (Williamson) - Xu and Ujiié, p. 519, pl. 10-6.

1994 Melonis barleeanus (Williamson) - Loeblich and Tappan, p. 159, pl. 347, figs. 15.

1998 Melonis barleeanum (Williamson) - Hess, pp. 84, 85, pl. 13, fig. 5 .

2000 Melonis barleeanum (Williamson) Ohkushi, Thomas, and Kawahata, p. 144, pl. 5 , fig. 6 .
Remarks. As mentioned by Ujiié (1990), the taxonomic relationships among species of Melonis, originally described as Nonionina barleeana Williamson, Nonion pacificum Cushman, Nonion nicobarensis Cushman, and Nonion parkerae Uchio are problematic. We regard $N$. parkerae Uchio (= Melonis uchioi, a new name to replace the junior homonym by Hasegawa, 1991), as a junior synonym of $M$. barleeanus.

Occurrence. Common in all the formations of the present study.

\section{Melonis pacificum (Cushman)}

Figures 32.10, 32.11

1924 Nonionina umbilicatula (Montagu) var. pacifica Cushman, p. 48, pl. 16, fig. 3.

1933 Nonion pacificum (Cushman) - Cushman(c), p. 44, pl. 10, fig. 9.

1950 Nonion pacificum (Cushman) - Asano (part 1), p. 3, text-figs. 13, 14.

Remarks. This species is characterized by almost non-limbate sutures and inflated chambers. As Ujiié (1990) mentioned the similarity to Melonis barleeanus, chamber inflation and lack of limbation might be a variety of that species, comparable to what has been observed for Hyalinea balthica or Cibicidoides pachyderma in the present study. We retain this nomenclature here, but stress that it should be examined further.

Occurrence. Sporadic in all the formations of the present study.

Melonis pompilioides (Fichtel and Moll)

Figures 32.12-32.14

1798 Nautilus pompilioides Fichtel and Moll, p. 31, pl. 2, figs. a-c.

1933 Nonion pompilioides (Fichtel and Moll) Cushman (c), pp. 41, 42, pl. 10, figs. 1, 2.

1941 Nonion pompilioides (Fichtel and Moll) LeRoy (Part 2), p. 78, pl. 6, figs. 28, 29.

1964 Nonion pompilioides (Fichtel and Moll) LeRoy, p. F27, pl. 10, figs. 10, 11.

1966 Melonis pompilioides (Fichtel and Moll) Belford, pp. 183, 184, pl. 30, figs. 17-20.

1968 Melonis pompilioides (Fichtel and Moll) Huang, p. 58, pl. 12, fig. 7.

1984 Melonis pompilioides (Fichtel and Moll) Rögl and Hansen, p. 30, pl. 2, figs. 1, 2, pl. 3, fig. 1.

1985 Melonis pompilioides (Fichtel and Moll) Papp and Schmid, p. 46, pl. 35, figs. 6, 7, pl. 36 , figs. $1-6$. 
1987 Melonis pompilioides (Fichtel and Moll) Loeblich and Tappan, pl. 696, figs. 7, 8.

1989 Melonis pompilioides (Fichtel and Moll) Hermelin, pp. 88, 89, pl. 17, figs. 13, 14; Inoue, p. 158, pl. 27, fig. 1.

1994 Melonis pompilioides (Fichtel and Moll) Jones, p. 108, pl. 109, figs. 10, 11; Loeblich and Tappan, p. 159, pl. 347, figs. 810.

Occurrence. Rare in the Onogoshi and Yonahama formations.

Genus PULLENIA Parker and Jones in Carpenter, Parker, and Jones, 1862

Pullenia bulloides (d'Orbigny)

Figures 32.15, 32.16

1846 Nonionina bulloides d'Orbigny, p. 107, pl. 5, figs. 9, 10.

1964 Pullenia bulloides (d'Orbigny) — LeRoy, p. F41, pl. 10, figs. 30, 31.

1965 Pullenia bulloides (d'Orbigny) - Todd, $\mathrm{p}$. 48, pl. 18, fig. 6 .

1977 Pullenia cf. bulloides (d'Orbigny) McCulloch, pp. 435, 436, pl. 171, fig. 2.

1984 Pullenia bulloides (d'Orbigny) - Rögl and Hansen, pl. 30, figs. 5, 6.

1985 Pullenia bulloides (d'Orbigny) - Papp and Schmid, p. 45, pl. 34, figs. 6-9.

1988 Pullenia bulloides (d'Orbigny) - Wang, Zhang, Zhao, Min, Bian, Zheng, Cheng, and Chen, p. 176, pl. 32, figs. 6, 7; Marle, p. 148 , pl. 3, fig. 4.

1989 Pullenia bulloides (d'Orbigny) - Hermelin, pp. 78,79 , pl. 15, figs. 4,5 .

1990 Pullenia bulloides (d'Orbigny) — Ujiié, pp. 42, 43, pl. 23, figs. $1,2$.

1994 Pullenia bulloides (d'Orbigny) - Jones, p. 92, pl. 84, figs. 11, 12.

1995 Pullenia bulloides (d'Orbigny) - Ujiié, p. 70, pl. 12, fig. 6.

1998 Pullenia bulloides (d'Orbigny) - Hess, p. 87, pl. 13, figs. 9, 10.

2000 Pullenia bulloides (d'Orbigny) - Ohkushi, Thomas, and Kawahata, p. 144, pl. 5, fig. 9.

2001 Pullenia bulloides (d'Orbigny) - Hayward, figs. 16-T, 16-U.

Remarks. Loeblich and Tappan (1994) identified spherical species of Pullenia quite similar to $P$. bulloides as Pullenia bikiniensis McCulloch (1977).
Pullenia bikiniensis is regarded a junior synonym of Pullenia quinqueloba (Reuss) in the present study. Pullenia bikiniensis of Loeblich and Tappan (1994) resembles $P$. bulloides in a broadly rounded periphery but is distinguished from the typical form in having four chambers in the ultimate whorl rather than five, and probably constitutes a different species.

Occurrence. Common in the Onogoshi, Yonahama and Minebari formations.

\section{Pullenia quinqueloba (Reuss)}

Figures 32.17, 32.18

1851 Nonionina quinqueloba Reuss, p. 71, pl. 5, fig. 31.

1944 Pullenia quinqueloba (Reuss) - LeRoy (Part 2), p. 90, pl. 4, figs. 18, 19.

1977 Pullenia bikiniensis McCulloch, p. 435, pl. 171, fig. 3.

1988 Pullenia quinqueloba (Reuss) - Marle, p. 148, pl. 3, fig. 5.

1989 Pullenia quinqueloba (Reuss) - Ōki, p. 149, pl. 20, fig. 7.

1990 Pullenia quinqueloba (Reuss) - Ujiié, p. 43, pl. 24, figs. $1-5$.

1994 Pullenia quinqueloba (Reuss) - Jones, p. 92, pl. 84, figs. 14, 15.

1994 Pullenia borealis Saidova - Loeblich and Tappan, p. 160, pl. 348, figs. $1-6$ (?non Pullenia borealis Saidova, 1975).

1998 Pullenia quinqueloba (Reuss) - Hess, p. 87, pl. 13, figs. 11, 12.

2000 Pullenia quinqueloba (Reuss) - Ohkushi, Thomas, and Kawahata, p. 144, pl. 5, fig. 4.

2002 Pullenia quinqueloba (Reuss) - Akimoto, Matsui, Shimokawa, and Furukawa, p. 24, pl. 62, fig. 1.

Occurrence. Sporadic in the Yonahama and Minebari formations.

Superfamily CHILOSTOMELLOIDEA Brady, 1881

Family CHILOSTOMELLIDAE Brady, 1881

Subfamily CHILOSTOMELLINAE Brady, 1881

Genus CHILOSTOMELLA Reuss in Cžjžek, 1849 Chilostomella cushmani Chapman

Figure 32.19

1941 Chilostomella cushmani Chapman, p. 177, pl. 8, fig. 9, pl. 9, fig. 6 .

1944 Chilostomella ovoidea Reuss - LeRoy (Part 1), p. 38, pl. 4, figs. 34, 35. 
1964 Chilostomella oolina Schwager - LeRoy, p. F41, pl. 2, fig. 39 (non Chilostomella oolina Schwager, 1878).

1978 Chilostomella cushmani Chapman Hofker, p. 45, pl. 5, fig. 6.

1988 Chilostomella ovoidea Reuss - Wang, Zhang, Zhao, Min, Bian, Zheng, Cheng, and Chen, pp. 176, 177, pl. 32, fig. 8 (non Chilostomella ovoidea Reuss, 1850).

1989 Chilostomella oolina Schwager - Inoue, pl. 32, fig. 12.

1990 Chilostomella cushmani Chapman - Ujiié, p. 41, pl. 22, figs. 3, 4 .

1992 Chilostomella oolina Schwager - Kaiho, pl. 7, figs. 7, 8; Kaiho and Nishimura, pl. 4, fig. 15.

1994 Chilostomella oolina Schwager - Jones, p. 61 , pl. 55, figs. 12-14, 17, 18.

1994 Chilostomella ovoidea Reuss - Loeblich and Tappan, pp. 160, 161, pl. 350, figs. 13.

2000 Chilostomella oolina Schwager Ohkushi, Thomas, and Kawahata, p. 139, pl. 2, fig. 15.

Remarks. Considerable confusion exists among C. cushmani, C. ovoidea, and C. oolina. Chilostomella oolina is distinguished from the other two in having a much more slender shape. Chilostomella ovoidea was originally described from the Miocene; the name has been applied by many workers for Recent specimens. Chilostomella ovoidea has a more inflated and ovoid form than C. cushmani.

Occurrence. Three specimens from a single sample of the Minebari Formation.

Family ALABAMINIDAE Hofker, 1951

Genus ALABAMINA Toulmin, 1941

Alabamina japonica (Asano)

Figure 32.20

1949 Pseudoparella japonica Asano, p. 429, text-figs. 2-4.

2004 Pseudoparella japonica Asano - Scott, Takayanagi, Hasegawa, and Saito, p. 31, figs. 11.218, 11.219.

Remarks. We follow Takayanagi and Hasegawa (1987) as far as the generic placement of this species is concerned. To the best of our knowledge, there are no other reports except of the two mentioned above, both using the original type specimens at Tohoku University. Therefore, the range of variation is unknown. Ujiié et al. (1983) regarded this species to be synonymous with Epistominella pacifica (Cushman), but that is a distinct species in a different genus.

Occurrence. A single specimen from the Minebari Formation.

Family OSANGULARIIDAE Loeblich and Tappan, 1964

Genus OSANGULARIA Brotzen, 1940

Osangularia culter (Parker and Jones)

Figures $32.21,32.22$

1865 Planorbulina farcata (Fichtel and Moll) var. ungeriana (d'Orbigny) subvar. culter Parker and Jones, pp. 382, 421, pl. 19, fig. 1.

1964 Osangularia bengalensis (Schwager) LeRoy, p. F38, pl. 9, figs. 32, 33 (non Anomalina bengalensis Schwager, 1866).

1965 Osangularia culter (Parker and Jones) Todd, pp. 25, 26, pl. 15, fig. 1.

1966 Osangularia culter (Parker and Jones) Belford, pp. 175, 176, pl. 35, figs. 1-5.

1968 Osangularia bengalensis (Schwager) Huang, p. 57, pl. 12, figs. 13, 14.

1988 Osangularia culter (Parker and Jones) Marle, p. 148, pl. 2, figs. 18-20.

1990 Osangularia bengalensis (Schwager) Ujiié, p. 49, pl. 28, fig. 7.

1992 Osangularia bengalensis (Schwager) Kaiho and Nishimura, pl. 4, fig. 17.

1994 Osangularia bengalensis (Schwager) Jones, p. 100, pl. 96, fig. 3.

1995 Osangularia bengalensis (Schwager) Ujiié, p. 71, pl. 13, fig. 1.

1998 Osangularia culter (Parker and Jones) Hess, p. 86, pl. 14, figs. 11, 12.

Remarks. This species has been listed as Osangularia bengalensis in many reports. Revets (1996b) discussed that the type "bengalensis" has supplementary trematopores on the apertural face and concluded that it belonged to the genus Cribroparella.

Occurrence. Common in the Yonahama and Minebari formations.

Family ORIDORSALIDAE Loeblich and Tappan, 1984

Genus ORIDORSALIS Andersen, 1961

Oridorsalis umbonatus (Reuss)

Figures $32.23,32.24$

1851 Rotalia umbonata Reuss, p. 75, pl. 5, fig. 35. 
1884 Truncatulina tenera Brady, p. 665, pl. 95, fig. 11.

1921 Pulvinulina umbonata (Reuss) - Cushman, pp. 339, 340, pl. 71, fig. 1.

1941 Eponides umbonatus (Reuss) - LeRoy (Part 2), p. 84, pl. 3, figs. 19-21.

1944 Eponides umbonatus (Reuss) - LeRoy (Part 1), p. 34, pl. 2, figs. 22-24.

1964 Pseudoeponides umbonatus (Reuss) LeRoy, p. F39, pl. 7, figs. 33-38.

1966 Oridorsalis umbonatus (Reuss) - Belford, pp. 172, 173, pl. 30, figs. $1-6$, textfigs. 22-4, 22-5.

1988 Oridorsalis umbonatus (Reuss) - Marle, p. 148, pl. 3, figs. 10, 15.

1989 Oridorsalis umbonatus (Reuss) - Inoue, p. 158, pl. 24, fig. 13.

1989 Oridorsalis tener (Brady) - Ōki, p. 149, pl. 20, fig. 8.

1990 Oridorsalis umbonatus (Reuss) Akimoto, p. 206, pl. 24, fig. 4; Ujiié, pp. 48, 49 , pl. 28 , figs. $1-6$, text-fig. 4 .

1994 Oridorsalis umbonatus (Reuss) - Xu and Ujiié, pp. 518, 519, figs. 10-1, 10-2; Akimoto, p. 285, pl. 3, fig. 5.

1994 Oridorsalis umbonata (Reuss) - Jones, p. 99, pl. 95, fig. 11, p. 104, pl. 105, fig. 2.

1995 Oridorsalis umbonatus (Reuss) - Ujiié, pl. 8, figs. 3, 4.

1998 Oridorsalis umbonatus (Reuss) - Hess, pp. 85, 86, pl. 14, figs. 9, 10.

2000 Oridorsalis umbonatus (Reuss) Ohkushi, Thomas, and Kawahata, p. 144, pl. 5, fig. 1.

2001 Oridorsalis umbonatus (Reuss) - Hayward, Carter, Grenfell, and Hayward, figs.16-R, 16-S.

Remarks. Ujiié (1990) distinguished Eponides tenerus (Brady) and Oridorsalis umbonatus (Reuss) based on the supplementary aperture in dorsal sutures. We regard these two as synonyms; there is variation in the development of supplementary apertures, which conforms with the description supplied by Loeblich and Tappan (1987).

Occurrence. Common in the Onogoshi, Yonahama, and Minebari formations.

Family HETEROLEPIDAE González-Dosono, 1969

Genus ANOMALINOIDES Brotzen, 1942
Anomalinoides glabratus (Cushman)

Figures 33.1, 33.2

1924 Anomalina glabrata Cushman, p. 39, pl. 12, figs. 5-7.

1941 Anomalina glabrata Cushman - LeRoy (Part 2), p. 88, pl. 3, figs. 16-18; LeRoy (Part 3), p. 119, pl. 2, figs. 19-21.

1944 Anomalina glabrata Cushman - LeRoy (Part 2), p. 92, pl. 6, figs. 16-18.

1959 Anomalina glabrata Cushman - Graham and Militante, p. 115, pl. 19, fig. 8.

1964 Anomalina glabrata Cushman - LeRoy, p. F43, pl. 6, figs. 8-10.

1966 Anomalina glabrata Cushman - Belford, p. 177 , pl. 32, figs. $1-7$.

1989 Anomalina glabrata Cushman - Ōki, pl. 21 , fig. 4.

Remarks. The genus Anomalina is problematic because d'Orbigny did not assign a holotype for its type species, A. punctulata, no specimens from the type area can be located, and d'Orbigny's original figures were not clear. Hansen and Rögl (1980) suggested that the use of this genus be suppressed, and we follow their convention here.

Occurrence. Common in the Onogoshi and Minebari formations, rare in the Yonahama Formation.

Anomalinoides globulosus (Chapman and Parr) Figures 33.3, 33.4

1937 Anomalina globulosa Chapman and Parr, p. 117, pl. 9, fig. 27.

1968 Anomalina globulosa Chapman and Parr - Huang, p. 55, pl. 12, fig. 17.

1989 Anomalinoides globulosa (Chapman and Parr) - Hermelin, pp. 84, 85, pl. 17, figs. 1, 5.

1992 Anomalinoides globulosa (Chapman and Parr) - Hatta and Ujiié (b), pp. 197, 198, pl. 43 , fig. 4.

1992 Anomalinoides globulosus (Chapman and Parr) - Kaiho, pl. 6, fig. 1.

1994 Anomalinoides globulosa (Chapman and Parr) - Akimoto, p. 283, pl. 3, fig. 13.

1994 Cibicidoides globulosus (Chapman and Parr) - Jones, p. 98, pl. 94, figs. 4, 5.

1994 Anomalinoides globulosus (Chapman and Parr) - Loeblich and Tappan, p. 162, pl. 354, figs. 1-13, pl. 355, figs. 4-13.

1998 Anomalinoides globulosus (Chapman and Parr) - Hess, p. 75, pl. 16, figs. 13, 14. 


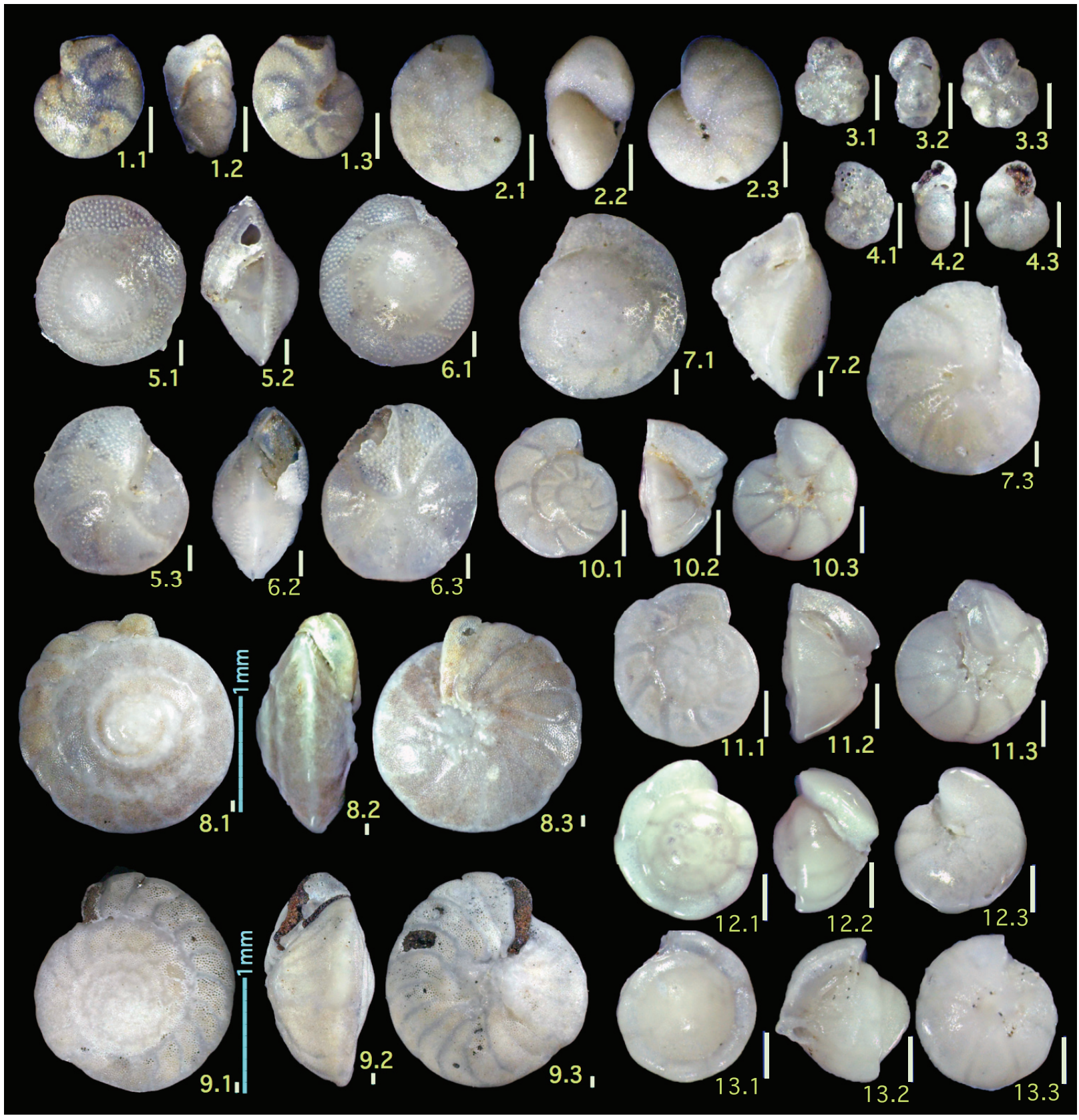

FIGURE 33. Family Heterolepidae and Gavelinellidae. All scale bars $=0.1 \mathrm{~mm}$, unless otherwise indicated. $\mathbf{1}, \mathbf{2}$. Anomalinoides glabratus (Cushman), 1: MPC-26127 from sample 3 (Onogoshi Formation, PL1); 2: MPC-26128 from sample 3-2-up-sand (Onogoshi F., PL1). 3, 4. Anomalinoides globulosus (Chapman and Parr), 3: MPC-26129, 4: MPC-26130, both from sample 10 (Onogoshi F., PL2). 5-7. Heterolepa dutemplei (d'Orbigny), 5: MPC-26317 and 6: MPC-26318, from sample MK01 (Yonahama F., PL2); 7: MPC-26319 from sample 18 (Oura F., ?PL1); 5: plano-convex, acute-periphery morphotype; 6: biconvex, round-periphery morphotype; 7: umbilico-convex, acute periphery morphotype. 8, 9. Heterolepa praecincta (Karrer), 8: MPC-26320 from sample 2-2 (Minebari F., PL5); 9: MPC-26321 from sample 6-2 (Yonahama F., PL2). 10, 11. Gyroidinoides altiformis (Stewart and Stewart), 10: MPC-26300 from sample 3 (Onogoshi F., PL1); 11: MPC-26301 from sample 18 (Oura F., ?PL1). 12, 13. Gyroidinoides broeckhiana (Karrer), 12: MPC-26302 from sample 5-2 (Minebari F., PL5); 13: MPC-26303 from sample MK01 (Yonahama F., PL2). 
Occurrence. Twenty-four specimens from a single sample of the Onogoshi Formation.

Genus HETEROLEPA Franzenau, 1884 Heterolepa dutemplei (d'Orbigny)

Figures 33.5-33.7

1846 Rotalina dutemplei d'Orbigny, p. 157, pl. 8, figs. $19-21$.

1950 Cibicides subhaidingerii Parr, p. 364, pl. 15, fig. 7.

1966 'Eponides' subhaidingeri (Parr) - Belford, pp. 129,130 , pl. 16 , figs. $7-13$.

1985 Heterolepa dutemplei (d'Orbigny) - Papp and Schmid, pp. 57, 58, pl. 46, figs. 5-9, p. 59 , pl. 50 , figs. $1-3$, p. 61 , pl. 52 , figs. $1-6$.

1988 Heterolepa dutemplei (d'Orbigny) Marle, p. 145, pl. 1, figs. 11-13.

1989 Heterolepa subhaidingerii (Parr) - Inoue, pl. 21, fig. 1, pl. 31, fig. 1.

1989 Cibicidoides? subhaidingerii (Parr) - Ōki, p. 152, pl. 22, fig. 1.

1990 Heterolepa subhaidingerii (Parr) Akimoto, p. 201, pl. 23, fig. 3.

1994 Cibicidoides subhaidingerii (Parr) Jones, p. 99, pl. 95, fig. 7.

1994 Heterolepa subhaidingerii (Parr) - Loeblich and Tappan, p. 163, pl. 359, figs. 113.

1996 Heterolepa dutemplei (d'Orbigny) Revets (b), p. 67, pl. 2, figs. 9-12.

2002 Heterolepa subhaidingerii (Parr) Akimoto, Matsui, Shimokawa, and Furukawa, pp. 19, 20, pl. 51, fig. 1.

Remarks. This is one of the more commonly found large benthic foraminifera in shallow to bathyal seas off Japanese Islands in the southwest. It shows a wide range of variation in the acuteness of the periphery and test convexity. Papp and Schmid (1985) reillustrated d'Orbigny's (1846) specimens of Rotalina Kalembergensis, Rotalina Haidingerii, and Rotalina Dutemplei, and concluded that $H$. dutemplei was a senior synonym.

Occurrence. Abundant in the Oura and Onogoshi formations, sporadic in the Yonahama and Minebari formations.

\section{Heterolepa praecincta (Karrer)}

Figures 33.8, 33.9

1868 Rotalina praecincta Karrer, p. 189, pl. 5, fig. 7.
1941 Eponides praecinctus (Karrer) - LeRoy (Part 1), p. 39, pl. 1, figs. 66-68; LeRoy (Part 2), p. 83, pl. 3, figs. 25-27.

1944 Eponides praecinctus (Karrer) - LeRoy (Part 1), p. 34, pl. 2, fig. 31-33, pl. 6, figs. 22-24, pl.7, figs. 25-27; LeRoy (Part 2), p. 89, pl. 5, figs. 4-6.

1966 'Eponides' praecinctus (Karrer) - Belford, pp. 125, 126, pl. 16, figs. 1-6.

1977 Cibicidoides (?) cf. praecinctus (Karrer) McCulloch, p. 446, pl. 152, fig. 11.

1988 Heterolepa praecincta (Karrer) - Wang, Zhang, Zhao, Min, Bian, Zheng, Cheng, and Chen, p. 179, pl. 33, fig. 13.

1994 Neoeponides praecinctus (Karrer) Jones, p. 99, pl. 95, figs. 1-3.

1994 Heterolepa praecincta (Karrer) - Loeblich and Tappan, p. 163, pl. 360, figs. 1-10.

Occurrence. Common in the Onogoshi and Minebari formations, rare in the Yonahama Formation.

Family GAVELINELLIDAE Hofker, 1956a

Subfamily GYROIDINOIDINAE Saidova, 1981

Genus GYROIDINOIDES Brotzen, 1942

Gyroidinoides altiformis (Stewart and Stewart)

Figures 33.10, 33.11

1930 Gyroidina soldanii d'Orbigny var. altiformis Stewart and Stewart, p. 67, pl. 9, fig. 2.

1959 Gyroidinoides altiformis (R.E. and K.C. Stewart) - Blow, pp. 160, 161, pl. 7, fig. 27.

1964 Gyroidina altiformis R.E. and K.C. Stewart — LeRoy, p. F37, pl. 7, figs. 7-9.

1966 Gyroidina acuta Boomgaart - Belford, pp. 165,167 , pl. 28 , figs. $1-9$, text-figs. 21-6, 21-7.

1968 Gyroidina acuta Boomgaart - Huang, p. 57, pl. 13, fig. 1 (non Gyroidina neosoldanii Brotzen var. acuta Boomgaart, 1949? MS).

1989 Gyroidina altiformis R.E. and K.C. Stewart - Hermelin, p. 81, pl. 15, figs. 13-15; Inoue, pl. 22, fig. 1.

1994 Gyroidina altiformis R.E. and K.C. Stewart - Akimoto, p. 284, pl. 5, fig. 1.

Remarks. Barbieri (1991) described two subspecies: Gyroidinoides altiformis altiformis (Stewart and Stewart) and Gyroidinoides altiformis acutus (Boomgaart). Our material matches feature of the subspecies altiformis.

Occurrence. Common in the Oura and Onogoshi formations, rare in the Minebari Formation. 


\section{Gyroidinoides broeckhiana (Karrer)}

Figures 33.12, 33.13

1878 Rotalina broeckhiana Karrer, p. 98, pl. 5, fig. 26.

1966 Gyroidina broeckhiana (Karrer) - Belford, pp. 167, 168, pl. 29, figs. $1-7$, text-figs. 21-10, 21-11.

1968 Gyroidina broeckhiana (Karrer) - Huang, p. 57 , pl. 13 , figs. $6,10$.

1998 Gyroidina broeckhiana (Karrer) - Hess, p. 82, pl. 15, figs. 10, 11.

Occurrence. Rare in the Yonahama Formation, common in the Minebari Formation.

Gyroidinoides aff. gemmus (Bandy)

Figures $34.1,34.2$

Compared with:

1953 Gyroidina gemma Bandy, p. 179, pl. 23, fig. 4.

Remarks. This species is closely related to Gyroidina gemma Bandy and Gyroidinoides kawagatai Ujiié (1995), but can be distinguished from both species by its radiate dorsal sutures and apertural slit-opening which reaches the umbilicus. We could not find a named species to refer this form to.

Occurrence. Rare in the Onogoshi, Yonahama and Minebari formations.

\section{Gyroidinoides soldanii (d'Orbigny)}

Figures 34.3, 34.4

1826 Gyroidina soldanii d'Orbigny, p. 278.

1936 Gyroidina neosoldanii Brotzen, p. 158.

1941 Gyroidina soldanii (d'Orbigny) - LeRoy (Part 1), p. 38, pl. 1, figs. 40-42; LeRoy (Part 2), p. 83, pl. 4, figs. 19-21.

1964 Gyroidina neosoldanii Brotzen - LeRoy, p. F37, pl. 7, figs. 4-6.

1977 Gyroidina cf. neosoldanii Brotzen McCulloch, pp. 371, 372, pl. 139, figs. 58.

1985 Gyroidina soldanii d'Orbigny - Papp and Schmid, p. 60, pl. 50, fig. 109.

1989 Gyroidina neosoldanii Brotzen - Hermelin, p. 81, pl. 15, figs. 16-18.

1990 Gyroidina neosoldanii Brotzen - Ujiié, p. 46, pl. 25, fig. 6, pl. 26, figs. 1, 2.

1992 Gyroidinoides neosoldanii (Brotzen) Kaiho, pl. 8, fig. 10; Kaiho and Nishimura, pl. 5 , fig. 8 .

1994 Gyroidinoides soldanii Brotzen - Jones, p. 106 , pl. 107 , figs. 6,7 .
1994 Gyroidina pulisukensis (Saidova) - Loeblich and Tappan, p. 164, pl. 363, figs. 17 (non Gyroidinus pulisukensis Saidova, 1975).

Remarks. Hansenisca soldanii (d'Orbigny) of Loeblich and Tappan $(1987,1994)$ does not appear to be conspecific with Gyroidina soldanii of Papp and Schmid (1985), who restudied the type specimens of d'Orbigny. Hansenisca soldanii of Loeblich and Tappan (1987, 1994) shows a broadly opened umbilicus and slit-like opening along the dorsal side of the ultimate whorl.

Occurrence. Common in the Onogoshi, Yonahama, and Minebari formations.

\section{Gyroidinoides sp. A}

Figures 34.5, 34.6

Description. Test small for the genus; plano-convex; umbilical side more convex; umbilicus almost closed but depressed; chambers slightly inflated; sutures slightly depressed, and those of dorsal side strongly curved backwards and those of umbilical side radiating; aperture at interio-marginal slit extending from periphery to umbilicus; surface smooth and very finely perforated.

Remarks. This species is distinguished from congeners in having strongly curved sutures on the dorsal side; however, its small size suggests that this species might represent the juvenile stage of another species of Gyroidinoides.

Occurrence. Sporadic in the Onogoshi, Yonahama, and Minebari formations.

Subfamily GAVELINELLINAE Hofker, 1956a Genus GYROIDINA d'Orbigny, 1826 Gyroidina nipponica Ishizaki

Figures 34.7, 34.8

1944 Gyroidina nipponica Ishizaki, p. 102, pl. 3, fig. 3.

1964 Gyroidina nipponica Ishizaki - LeRoy, p. F37, pl. 7, figs. 13-15.

1988 Gyroidina nipponica Ishizaki - Wang, Zhang, Zhao, Min, Bian, Zheng, Cheng, and Chen, p. 177, pl. 32, figs. 9, 10.

1989 Gyroidinoides nipponicus (Ishizaki) - Ōki, pp. 150, 151, pl. 21, fig. 3; Inoue, pl. 22, fig. 2, pl. 31, fig. 11.

1990 Gyroidinoides nipponicus (Ishizaki) Akimoto, p. 201, pl. 21, fig. 4; Ujiié, p. 47, pl. 27, fig. 1.

1994 Gyroidinoides nipponicus (Ishizaki) Akimoto, p. 284, pl. 3, fig. 4.

Remarks. This species has an involute umbilicus and is different from Gyroidinoides. 


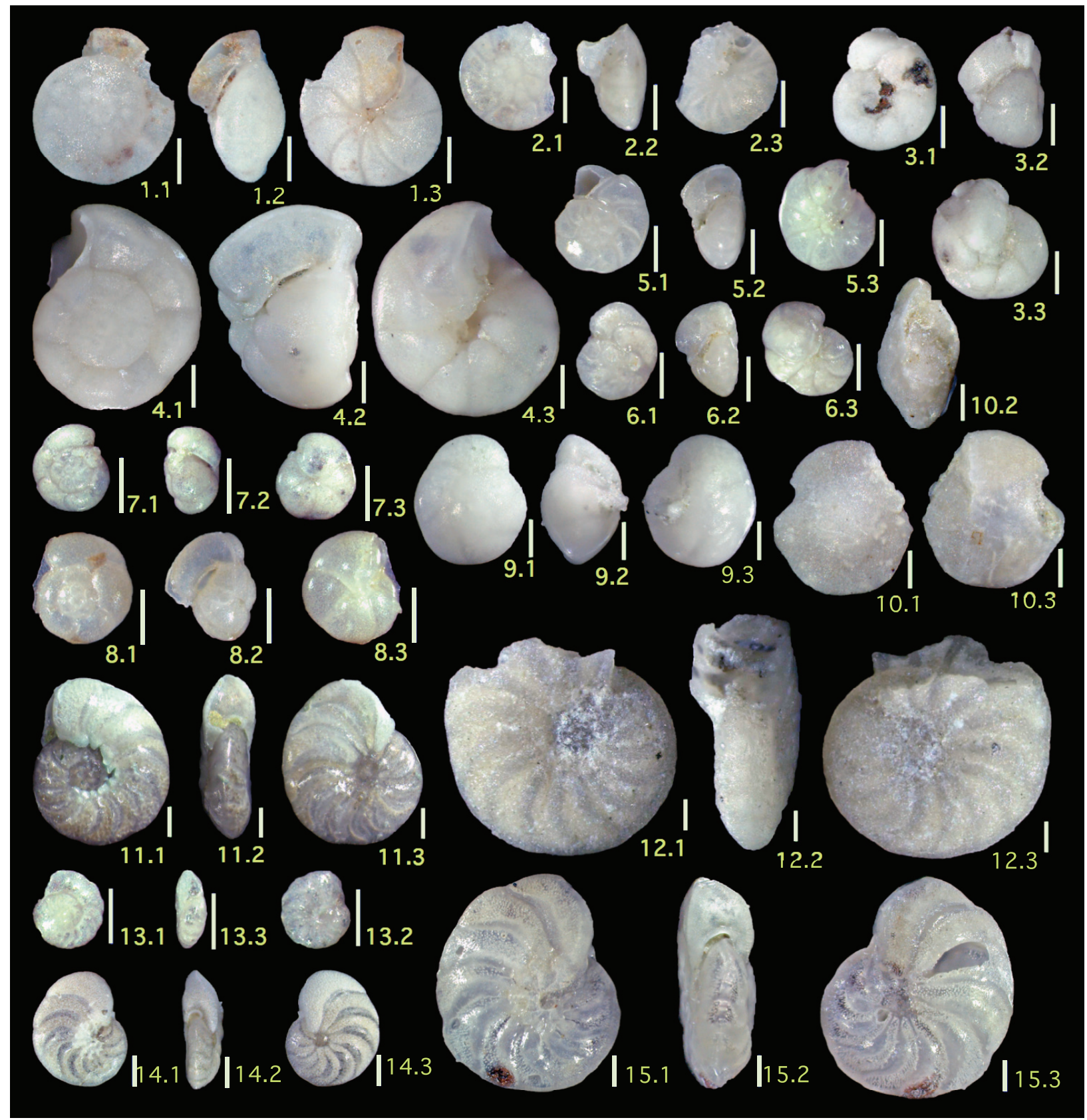

FIGURE 34. Family Gavelinellidae. All scale bars $=0.1 \mathrm{~mm} .1$, 2. Gyroidinoides aff. gemmus (Bandy), 1: MPC-26304 from sample 17 (Yonahama Formation, PL2); 2: MPC-26305 from sample MK01 (Yonahama F., PL2). 3, 4. Gyroidinoides soldanii (d'Orbigny), 3: MPC-26306 from sample 3-2-low-silt (Onogoshi F., PL1); 4: MPC-26307 from sample MK01 (Yonahama F., PL2). 5, 6. Gyroidinoides sp.A, 5: MPC-26308 from sample 3-2-low-silt (Onogoshi F., PL1); 6: MPC-26309 from sample 8 (Yonahama F., PL4). 7, 8. Gyroidina nipponica Ishizaki, 7: MPC-26296 from sample 11 (Onogoshi F., ?PL1); 8: MPC-26297 from sample MK02B (Minebari F., PL5). 9, 10. Gyroidina sp.A, 9: MPC-26298 from sample 15 (Yonahama F., PL3); 10: MPC-26299 from sample MK02A-2 (Minebari F., PL5). 11, 12. Hanzawaia asterizana (Fichtel and Moll), 11: MPC-26310 from sample 2 (Minebari F., PL5); 12: MPC-26311 from sample MK02A (Minebari F., PL5). 13. Hanzawaia cf. coronata (Heron-Allen and Earland), MPC-26312 from sample MK01 (Yonahama F., PL2). 14, 15. Hanzawaia nipponica Asano, 14: MPC-26313 from sample 4 (Minebari F., PL5); 15: MPC26314 from sample 18 (Oura F., ?PL1). 
Occurrence. Common in the Onogoshi, Yonahama, and Minebari formations.

Gyroidina sp. A

Figures $34.9,34.10$

Description. Test biconvex, umbilical side slightly more convex; six to seven chambers in final whorl; sutures on dorsal side gently curved backwards and radiating on umbilical side, not depressed; periphery round to subacute; umbilicus closed; aperture interiomarginal in the middle of apertural face; surface smooth and finely perforated.

Remarks. Due to poor preservation of specimens, we tentatively distinguish this species as $\mathrm{sp}$. A.

Occurrence. A single specimen each from the Yonahama and Minebari formations.

Genus HANZAWAIA Asano, 1944

Hanzawaia asterizana (Fichtel and Moll)

Figures $34.11,34.12$

1798 Nautilus asterizans Fichtel and Moll, p. 37, pl. 3, figs. e-h.

1949 Hanzawaia tagaensis Asano, p. 430, textfigs. 2-28-2-32.

1951 Hanzawaia tagaensis Asano - Asano (part 13), p. 16, text-figs. 27-29.

1964 Anomalina bradyi Said - LeRoy, pp. F43, F44, pl. 6, figs. 12-14 (non Anomalina brady Said, 1949).

1984 Hanzawaia asterizans (Fichtel and Moll) Rögl and Hansen, pp. 34, 35, pl. 8, figs. $1-3$, text-fig 9 .

2000 Hanzawaia asterizans (Fichtel and Moll) Scott, Takayanagi, Hasegawa, and Saito, p. 26, figs. 9.172, 9.173.

Remarks. Hanzawaia tagaensis, a junior synonym of this species, was originally described from the Miocene of Fukushima, but is also found in Recent sediments as mentioned by Asano (1951).

Occurrence. Rare in the Yonahama and Minebari formations.

Hanzawaia cf. coronata (Heron-Allen and Earland) Figure 34.13

Compared with:

1994 Hanzawaia coronata (Heron-Allen and Earland) - Loeblich and Tappan, p. 164, pl. 366, figs. 1-15.

Remarks. Our single specimen is a small juvenile; critical identification is difficult.

Occurrence. Yonahama Formation.
Hanzawaia nipponica Asano

Figures $34.14,34.15$

1944 Hanzawaia nipponica Asano, p. 99, pl. 4, figs. 1, 2.

1964 Hanzawaia nipponica Asano - LeRoy, p. F46, pl. 9, figs. 28, 29.

1988 Hanzawaia nipponica Asano - Wang, Zhang, Zhao, Min, Bian, Zheng, Cheng, and Chen, p. 178, pl. 33, figs. 1-5; Marle, p. 145, pl. 1, figs. 19, 20.

1989 Hanzawaia nipponica Asano - Ōki, p. 152, pl. 22, fig. 3; Inoue, pl. 20, fig. 10.

1990 Hanzawaia nipponica Asano - Akimoto, p. 201, pl. 20, fig. 4.

1994 Hanzawaia nipponica Asano - Loeblich and Tappan, p. 164, pl. 363, figs. 8-13.

1996 Hanzawaia nipponica Asano - Revets (b), pp. 66,67 , pl. 2, figs. $5-8$.

Occurrence. Abundant in the Oura Formation, sporadic in the Onogoshi and Minebari formations. Superfamily ROTALIOIDEA Ehrenberg, 1839

Family ROTALIIDAE Ehrenberg, 1839

Subfamily CUVILLIERININAE Loeblich and Tappan, 1964

Genus PARAROTALIA Le Calvez, 1949

Pararotalia cf. nipponica (Asano)

Figures 35.1, 35.2

Compared with:

1936 Rotalia nipponica Asano (b), p. 614, pl. 31, fig. 2.

Remarks. The present specimens are juvenile, and not suited for critical identification. The juvenile stage of Pararotalia nipponica has peripheral spines which disappear in the adult stage (e.g., Ujiié, 1966).

Occurrence. Two specimens from a single sample of Minebari Formation.

Subfamily AMMONIINAE Saidova, 1981

Genus AMMONIA Brünnich, 1772

Ammonia maruhasii (Kuwano)

Figure 35.3-35.6

1950 Rotalia maruhasii Kuwano, pp. 314, 315, text-figs. 2, 8.

1968 Ammonia ketienziensis angulata (Kuwano) - Huang, p. 55, pl. 12, figs. 8a, b (non Streblus ketienziensis Ishizaki, 1948).

1988 Ammonia maruhasii (Kuwano) - Wang, Zhang, Zhao, Min, Bian, Zheng, Cheng, 
and Chen, pp. 166, 167, pl. 26, figs. 14, 15, pl. 27, fig. 11.

Remarks. High trochospiral variation is included in this species. This species is distinguished from Rotalia ketienziensis Ishizaki in the lesser number of chambers, radial sutures, and less developed umbilical ornament. It also distinguished from Rotalia ketienziensis angulata Kuwano in having a more rounded periphery and radial sutures. It might be a Pliocene ancestral form of these modern Ammonia species.

Occurrence. Abundant in the Oura and Onogoshi formations, common in the Yonahama and Minebari formations.

\section{Ammonia parkinsoniana (d'Orbigny)}

Figures 35.7, 35.8

1839 Rosalina parkinsoniana d'Orbigny (a), p. 99, pl. 4, figs. 25-27.

1959 "Rotalia" parkinsoniana (d'Orbigny) Graham and Militante, p. 101, pl. 15, fig. 11.

1994 Ammonia parkinsoniana (d'Orbigny) Loeblich and Tappan, p. 165, pl. 368, figs. 7-16.

Remarks. We tentatively assign this small low-trochospiral Ammonia to the present species mainly based on comparison with figures of Loeblich and Tappan (1994). Ammonia is one of the most taxonomically complex groups, and we must await detailed taxonomic studies such as the one performed by Hayward et al. (2004) using a molecular approach.

Occurrence. Common in the upper part of Yonahama Formation and the Minebari Formation.

Genus ASTEROROTALIA Hofker, 1950 Asterorotalia pulchella (d'Orbigny)

Figures 35.9, 35.10

1839 Rotalina (Calcarena) pulchella d'Orbigny (a), p. 80.

1933 Rotalia trispinosa Thalmann, p. 248.

1951 Rotalia trispinosa Thalmann - Asano (part 14), p. 17, text-fig. 127

1964 Asterorotalia trispinosa (Thalmann) LeRoy, p. F39, pl. 6, figs. 18, 19.

1977 Asterorotalia cf. trispinosa (Thalmann) McCulloch, pp. 425, 426, pl. 152, figs. $1-$ 3.

1992 Asterorotalia trispinosa (Thalmann) Kadar, pp. 250, 253, fig. 6-8.

1994 Asterorotalia pulchella (d'Orbigny) Jones, p. 114, pl. 115, fig. 8.
Occurrence. Abundant in the Oura Formation, sporadic in the Onogoshi and Minebari formations.

Genus ROTALINOIDES Saidova, 1975

Rotalinoides compressiuscula (Brady)

Figures 35.11, 35.12

1884 Rotalia papillosa var. compressiuscula Brady, p. 708, pl. 107, figs. 1, 3.

1988 Ammonia compressiuscula (Brady) Wang, Zhang, Zhao, Min, Bian, Zheng, Cheng and Chen, p. 167, pl. 27, fig. 3, pl. 34, figs. 6-10.

1994 Rotalinoides compressiuscula (Brady) Jones, p. 106, pl. 107, figs. 1, 3.

2001 Pseudorotalia compressiusculus (Brady) — Kawagata, p. 112, fig. 16-6.

2006 Pseudorotalia gaimardii compressiuscula (Brady) - Takata, Irizuki, and Ishida, pl. 2, fig. 2.

Occurrence. Abundant in the Oura Formation, sporadic in the Minebari Formation.

Rotalinoides gaimardii (Fornasini)

Figures 36.1, 36.2

1826 Rotalia (Turbinulina) gaimardii d'Orbigny, p. 275 (nomen nudum).

1884 Rotalia papillosa Brady, p. 708, pl. 106, fig. 9.

1906 Turbinulina gaimardii Fornasini, pp. 67, 70.

1951 Rotalia cf. papillosa Brady - Asano (part 14), p. 15, text-figs. 118, 119.

1966 Pseudorotalia gaimardi (d'Orbigny) - Belford, pp. 115, 116, pl. 20, figs. 5-11.

1987 Rotalinoides gaimardii (d'Orbigny) - Loeblich and Tappan, pl. 773, figs. 1-8.

1988 Ammonia gaimardii (d'Orbigny) - Marle, p. 139 , pl. 4, figs. 4,5 .

1989 Pseudorotalia gaimardii (d'Orbigny) - Ōki, p. 133 , pl. 16, fig. 2; Inoue, pp. 153, 154, pl. 20, fig. 1.

1990 Pseudorotalia gaimardii (d'Orbigny) Akimoto, p. 208, pl. 21, fig. 2.

1992 Asterorotalia gaimardi (d'Orbigny) Kadar, p. 252, fig. 8-3.

1994 Rotalinoides gaimardii (Fornasini) Jones, p. 106, pl. 106, fig. 9

1994 Asterorotalia gaimardi (d'Orbigny) - Loeblich and Tappan, p. 166, pl. 372, figs. 17.

Occurrence. Sporadic in the Oura, Onogoshi, and Minebari formations. 


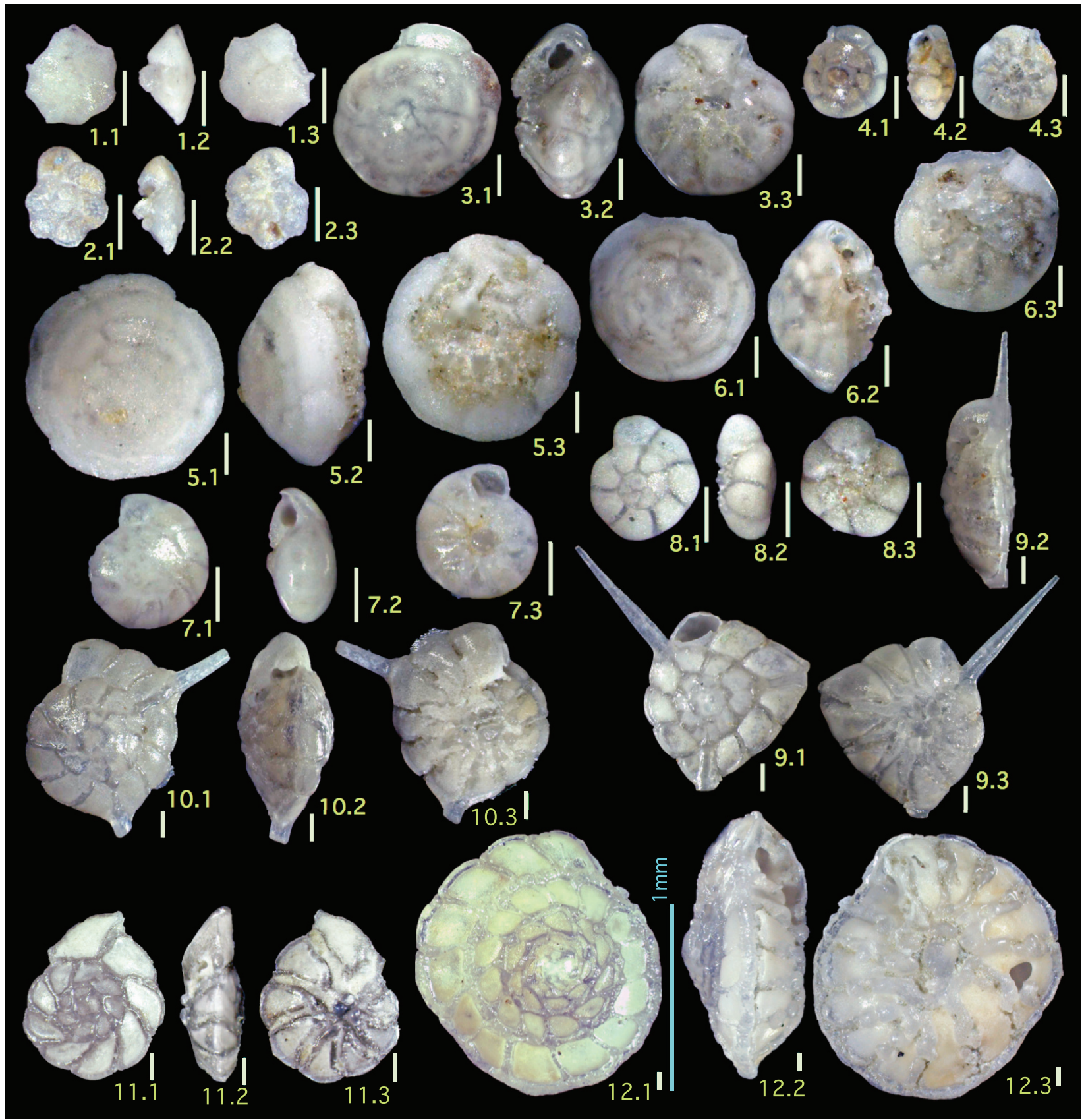

FIGURE 35. Family Rotaliidae. All scale bars $=0.1 \mathrm{~mm}$, unless otherwise indicated. 1, 2. Pararotalia cf. nipponica (Asano), 1: MPC-26407, 2: MPC-26408, both from sample MK02B (Minebari Formation, PL5). 3-6. Ammonia maruhasii (Kuwano), 3: MPC-26112 from sample 18 (Oura F., ?PL1); 4: MPC-26113 from sample MK02B (Minebari F., PL5); 5: MPC-26114 from sample 3-2-up-sand (Onogoshi F., PL1); 6: MPC-26115 from sample MK01 (Yonahama F., PL2); 5 and 6, high trochospiral variety. 7, 8. Ammonia parkinsoniana (d'Orbigny), 7: MPC-26116 from sample 14 (Yonahama F., PL4); 8: MPC-26117 from sample 14-2 (Yonahama F., PL4). 9, 10. Asterorotalia pulchella (d'Orbigny), 9: MPC-26134 from sample 10 (Onogoshi F., PL2); 10: MPC-26135 from sample 18 (Oura F., ?PL1). 11, 12. Rotalinoides compressiuscula (Brady), 11: MPC-26466 from sample 5-2 (Minebari F., PL5); 12: MPC-26467 from sample 18 (Oura F., ?PL1). 
Family ELPHIDIIDAE Galloway, 1933

Subfamily ELPHIDIINAE Galloway, 1933

Genus CRIBROELPHIDIUM Cushman and

Brönnimann, 1948

Cribroelphidium clavatum (Cushman)

Figures $36.3,36.4$

1930 Elphidium incertum (Williamson) var. clavatum Cushman, p. 20, pl. 7, fig. 10.

1992 Elphidium clavatum Cushman - Ōki and Yamamoto, p. 200, figs. 5-6.

Remarks. Genus Cribroelphidium is distinguished from Elphidium in rounded and perforate periphery. Cribrate apertural pores are based on the experience of the first author appears also in Elphidium.

Occurrence. Sporadic in the Minebari Formation.

Cribroelphidium subincertum (Asano)

Figures 36.5, 36.6

1950 Elphidium subincertum Asano (part 1), p. 10, text-figs. 56, 57.

1988 Cribroelphidium subincertum (Asano) Wang, Zhang, Zhao, Min, Bian, Zheng, Cheng, and Chen, pp. 168, 169, pl. 28, figs. 1 -4.

1989 Elphidium subincertum Asano - Ōki, pl. 16, fig. 10.

2000 Cribrononion subincertum (Asano) - Kim and Kucera, fig. 3.6.

Occurrence. Rare in the Yonahama and Minebari formations.

\section{Cribroelphidium sp. A \\ Figures 36.7, 36.8}

Description. Test small for the genus, planispiral and involute, both sides almost parallel, five chambers in final whorl; chambers inflated; sutures depressed and retral process appearing as small pores; umbilical area filled by shell material; periphery broadly rounded; aperture interiomarginal slit; surface smooth and moderately perforated.

Remarks. We could not find a named species to refer this to. It is characterized mainly by the small number of chambers in a whorl and the inflated, thick test.

Occurrence. Rare in the Yonahama and Minebari formations.

Genus ELPHIDIUM de Montfort, 1808

Elphidium advenum (Cushman)

Figures 36.9, 36.10

1922 Polystomella advena Cushman (a), p. 56, pl. 9, figs. 11, 12 .
1933 Elphidium advenum (Cushman) - Cushman (c), p. 50, pl. 12, figs. 1-3.

1937 Elphidium subcrispum Nakamura, p. 139, pl.11, fig. 8.

1959 Elphidium advena (Cushman) - Graham and Militante, p. 73, pl. 11, figs. 7, 8 .

1964 Elphidium tikutoense Nakamura - LeRoy, p. F28, pl. 10, figs. 3, 4 (non Elphidium tikutoense Nakamura, 1937).

1977 Elphidium(?) singaporense McCulloch, p. 224, pl. 97, fig. 2.

1988 Elphidium advenum (Cushman) - Wang, Zhang, Zhao, Min, Bian, Zheng, Cheng, and Chen, p. 170, pl. 29, figs. 1, 2; Marle, p. 143, pl. 1, fig. 6.

1989 Elphidium advenum (Cushman) - Ōki, pp. 133, 134, 173, pl. 16, fig. 2; Inoue, pl. 20 , fig. 5 .

1990 Elphidium advena (Cushman) - Akimoto, p. 197, pl. 7, fig. 1.

1992 Elphidium advenum (Cushman) - Hatta and Ujiié (b), p. 203, pl. 49, figs. 3, 4; Ōki and Yamamoto, p. 200, fig. 5-5.

1994 Elphidium advenum (Cushman) - Jones, p. 108, pl. 110, fig. 1; Loeblich and Tappan, p. 168 , pl. 379 , figs. $1-4$.

2000 Elphidium advenum (Cushman) - Kim and Kucera, fig. 3.7.

Occurrence. Common in the Minebari Formation. Elphidium crispum (Linnaeus)

Figures $36.11,36.12$

1758 Nautilus crispus Linnaeus, p. 709.

1933 Elphidium crispum (Linné) - Cushman (c), p. 47, pl. 11, fig. 4.

1944 Elphidium fax barbarense Nicol, p. 178, pl. 29, figs. 10-12.

1964 Elphidium fax barbarense Nicol — LeRoy, p. F28, pl. 10, figs. 1, 2.

1984 Elphidium crispum (Linné) - Rögl and Hansen, p. 37, pl. 8, fig. 7, text-fig. 10.

1985 Elphidium crispum (Linné) - Papp and Schmid, pp. 50, 51, pl. 40, figs. 5-8.

1989 Elphidium crispum (Linné) - Ōki, pp. 134, 135, pl. 16, fig. 4 ; Inoue, pl. 30 , fig. 5 .

1990 Elphidium crispum (Linné) - Ujiié, pl. 32, fig. 5.

1992 Elphidium crispum (Linné) - Hatta and Ujiié (b), p. 203, pl. 49, fig. 5. 


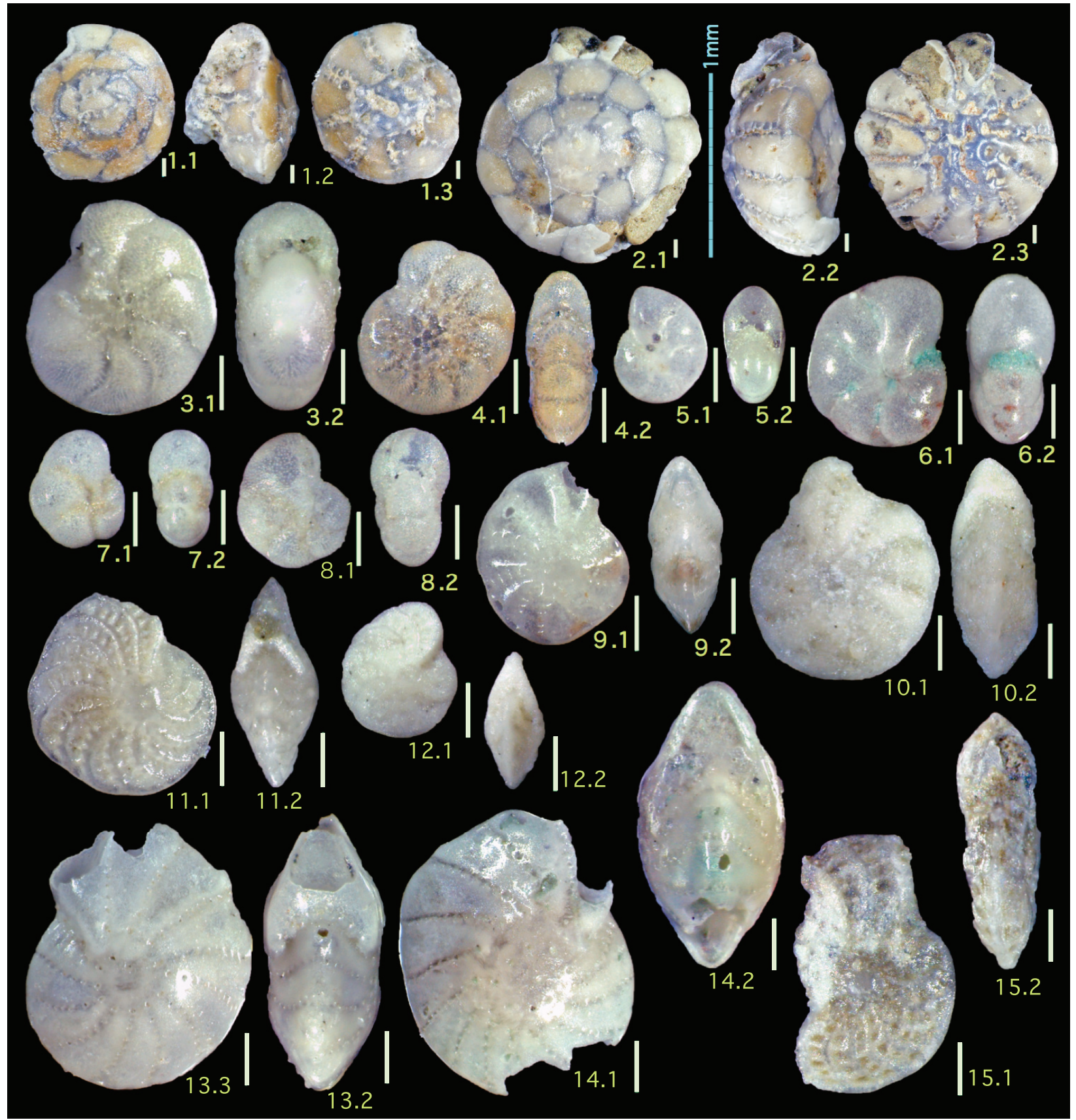

FIGURE 36. Family Rotaliidae and Elphidiidae. All scale bars $=0.1 \mathrm{~mm}$, unless otherwise indicated. 1, 2. Rotalinoides gaimardii (Fornasini), 1: MPC-26468 from sample 3-2-up-sand (Onogoshi Formation, PL1); 2: MPC-26469 from sample 18 (Oura F., ?PL1). 3, 4. Cribroelphidium clavatum (Cushman), 3: MPC-26210 from sample 2 (Minebari F., PL5); 4: MPC-26211 from sample MK02B (Minebari F., PL5). 5, 6. Cribroelphidium subincertum (Asano), 5: MPC-26212 from sample 1 (Minebari F., PL5); 6: MPC-26213 from sample 1-2 (Minebari F., PL5). 7, 8. Cribroelphidium sp.A, 7: MPC-26214 from sample 14 (Yonahama F., PL4); 8: MPC-26215 from sample 14-2 (Yonahama F., PL4). 9, 10. Elphidium advenum (Cushman), 9: MPC-26232 from sample 5 (Minebari F., PL5); 10: MPC-26233 from sample MK02A (Minebari F., PL5). 11, 12. Elphidium crispum (Linnaeus), 11: MPC-26234 from sample 2 (Minebari F., PL5); 12: MPC26235 from sample 16 (Yonahama F., PL3). 13, 14. Elphidium aff. neosimplex (McCulloch), 13: MPC-26236 from sample 11 (Onogoshi F., ?PL1); 14: MPC-26237 from sample 18 (Oura F., ?PL1). 15. Elphidium planulatum (Lamarck), MPC-26238 from sample 5-2 (Minebari F., PL5); broken specimen. 
1994 Elphidium crispum (Linné) - Jones, p. 109, pl. 110, figs. 6, 7; Loeblich and Tappan, pp. 168,169 , pl. 378 , figs. $4-6$.

Occurrence. Two specimens each from samples of the Yonahama and Minebari formations.

\section{Elphidium aff. neosimplex McCulloch}

Figures $36.13,36.14$

Compared with:

1977 Elphidium neosimplex McCulloch, p. 233, pl. 97, fig. 9.

1994 Elphidium neosimplex McCulloch - Loeblich and Tappan, p. 169, pl. 381, figs. 611.

Remarks. The present form closely resembles $E$. neosimplex in the lenticular morphology and pitted retral process, yet it is distinguished by its large test size, numerous chambers, and non-depressed umbilicus.

Occurrence. Rare in the Oura and Onogoshi formations.

\section{Elphidium planulatum (Lamarck) Figure 36.15}

1798 Nautilus strigilatus varietus $\alpha$ Fichtel and Moll, p. 68, pl. 10, figs. e-g.

1822 Polystomella planulara Lamarck, p. 625.

1941 Elphidium aff. jenseni (Cushman) LeRoy (Part 2), p. 78, pl. 6, figs. 32, 33.

1984 Elphidium planulatum (Lamarck) - Rögl and Hansen, pp. 49,50 , pl. 13, figs. 7, 8 , pl. 14, figs. 1, 3, 4, text-fig. 18A.

Remarks. This species is characterized by a strongly compressed test with numerous chambers. Material from Miyakojima Island is broken and suggests transportation from shallower seas.

Occurrence. A single specimen from the Minebari Formation.

\section{ACKNOWLEDGMENTS}

Haruna Koike prepared study materials through the Graduate and Master Course of Faculty of Education at Shizuoka University. The authors are indebted to the faculty staff for fruitful advice and encouragement. We express our special thanks to Dr. J. Jagt and Dr. E. Jagt-Yazykova for help us improving manuscript. We also thank anynymous reviewers for their valuable comments.

\section{REFERENCES}

Akimoto, K. 1990. Distribution of Recent benthic foraminiferal faunas in the Pacific off southwest Japan and around Hachijojima Island. Science Reports of the Tohoku University, Second Series (Geology), 60:139223.

Akimoto, K. 1994. Cenozoic benthic foraminiferal biostratigraphy, paleobathymetry, paleoenvironments and paleoceanography of the New Hebrides Island Arc and North d'Entrecasteaux Ridge area, p. 265291. In Green, H.G., Collot, J.-Y., Stokking, L.B., and Crawford, A.J. (eds.), Proceedings of the Ocean Drilling Program, Scientific Results, 134, College Station, TX (Ocean Drilling Program).

Akimoto, K., Matsui, C., Shimokawa, A., and Furukawa, K. 2002. Atlas for Holocene benthic foraminifers of Shimabara Bay, Kyushu, Southwest Japan. The Kagoshima University Museum Monographs, no. 2.

Albani, A.D. 1974. New benthonic Foraminiferida from Australian waters. Journal of Foraminiferal Research, 4:33-39.

Andersen, H.V. 1961. Genesis and paleontology of the Mississippi River mudlumps, Part II. Foraminifera of the mudlumps, lower Mississippi River delta. Louisiana Department of Conservation, Geological Bulletin, 35:1-208.

André, A., Weiner, A., Quillévéré, F., Aurahs, R., Morard, R., Douady, C.J., Garidel-Thoron, T. de, Escarguel, G., Vargas, C.de, and Kucera, M. 2013. The cryptic and the apparent reversed: lack of genetic differentiation within the morphologically diverse plexus of the planktonic foraminifer Globigerinoides sacculifer. Paleobiology, 39:21-39.

Aoki, N. 1968. Benthonic foraminiferal zonation of the Kazusa Group, Boso Peninsula. Transactions and Proceedings of the Palaeontological Society of Japan, New Series, no. 70:238-266.

Asano, K. 1936a. Pseudononion, a new genus of Foraminifera found in Muraoka-mura, Kamakura-gori, Kanagawa Prefecture. The Journal of the Geological Society of Japan, 43:347-348.

Asano, K. 1936b. Foraminifera from Muraoka-mura, Kamakura-gori, Kanagawa Prefecture. (Studies on the fossil Foraminifera from the Neogene of Japan, Part 1). The Journal of the Geological Society of Japan, 43:603-622.

Asano, K. 1936c. New foraminifera from the Kakegawa district, Tôtômi, Japan. (Studies on the fossil foraminifera from the Neogene of Japan, Part 4). Japanese Journal of Geology and Geography, 13:325-331.

Asano, K. 1938a. Japanese fossil Nodosariidae, with notes on the Frondiculariidae. Science Report of the Tohoku Imperial University, second series (Geology), 19:179-220.

Asano, K. 1938b. On the Japanese species of Nonion and its allied genera. The Journal of the Geological Society of Japan, 45:592-599. 
Asano, K. 1938c. On the Japanese species of Bolivina and its allied genera. The Journal of the Geological Society of Japan, 45:600-609.

Asano, K. 1938d. On the Japanese species of Uvigerina and its allied genera. The Journal of the Geological Society of Japan, 45:609-618.

Asano, K. 1944. Hanzawaia, a new genus of foraminifera, from the Pliocene of Japan. The Journal of the Geological Society of Japan, 51:97-99. (In Japanese with English abstract)

Asano, K. 1949. New Miocene foraminifera from Japan. Journal of Paleontology, 23:423-430.

Asano, K. 1950-1952. Illustrated Catalogue of Japanese Tertiary Smaller Foraminifera, Parts 1-15 and Supplement 1. Hosokawa Printing Company, Tokyo.

Asano, K. 1950. Japanese Tertiary species of Gaudryina and Gaudryinella. Short Papers from the Institute of Geology and Paleontology, Tohoku University, 1:510.

Asano, K. 1953. Miocene Foraminifera from the Noto Peninsula, Ishikawa Prefecture. Short Papers from the Institute of Geology and Paleontology, Tohoku University, 5:1-21.

Asano, K. 1956a. The Foraminifera from the adjacent seas of Japan, collected by S.S. Soyo-maru, 19221930: Part 1 Nodosariidae. Science Reports of the Tohoku University, second series (Geology), 27:1-55.

Asano, K. 1956b. The Foraminifera from the adjacent seas of Japan, collected by S.S. Soyo-maru, 19221930: Part 2. Miliolidae. Science Reports of the Tohoku University, second series (Geology), 27:5783.

Asano, K. 1957. The Foraminifera from the adjacent seas of Japan, collected by S.S. Soyo-maru, 19221930: Part 3. Planktonic Foraminifera. Science Reports of the Tohoku University, second series (Geology), 28:1-26.

Asano, K. 1958. The Foraminifera from the adjacent seas of Japan, collected by S.S. Soyo-maru, 19221930: Part 4. Buliminidae. Science Reports of the Tohoku University, second series (Geology), 29:1-41.

Asano, K. and Nakamura, M. 1937. On the Japanese species of Cassidulina. Japanese Journal of Geology and Geography, 14(2-3):143-153.

Bandy, O.L. 1953. Ecology and paleoecology of some California foraminifera: Part 1, The frequency distribution of Recent Foraminifera off California; Part 2, Foraminiferal evidence of subsidence rates in the Ventura Basin. Journal of Paleontology, 27:161-182.

Bandy, O.L. 1972. Origin and development of Globorotalia (Turborotalia) pachyderma (Ehrenberg). Micropaleontology, 18:294-318.

Bandy, O.L., Frerichs, W.E., and Vincent, E. 1967. Origin, development, and geologic significance of $\mathrm{NeO}-$ globoquadrina Bandy, Freichs, and Vincent, gen. nov. Contributions from the Cushman Foundation for Foraminiferal Research, 18:152-157.
Banner, F.T. and Blow, W.H. 1959. The classification and stratigraphical distribution of the Globigerinaceae. Palaeontology, 2:1-27.

Banner, F.T. and Blow, W.H. 1967. The origin, evolution and taxonomy of the foraminiferal genus Pulleniatina Cushman, 1927. Micropaleontology, 13:133-162.

Barbieri, R. 1991. Phenotypic variation in Gyroidinoides altiformis (Stewart \& Stewart) and Gyroidinoides subangularis (Plummer) (Foraminifera). Journal of Micropalaeontology, 9:233-238.

Barker, R.W. 1960. Taxonomic notes on the species figured by H.B. Brady in his report on the foraminifera dredged by $\mathrm{H}$. M. S. Challenger during the years 1873-1876. Society of Economic Paleontologists and Mineralogists, Special Publication, 9:1-238.

Bé, A.W.H. 1980. Gametogenic calcification in a spinose planktonic foraminifer, Globigerinoides sacculifer (Brady). Marine Micropaleontology, 5:283-310.

Belford, D.J. 1962. Miocene and Pliocene planktonic foraminifera, Papua-New Guinea. Commonwealth of Australia, Department of National Development, Bureau of Mineral Resources, Geology and Geophysics, Bulletin, 62-1.

Belford, D.J. 1966. Miocene and Pliocene smaller foraminifera from Papua and New Guinea. Commonwealth of Australia, Department of National Development, Bureau of Mineral Resources, Geology and Geophysics, Bulletin, 79.

Bergami, C., Captondi, L., Langone, L., Giglio, F., and Ravaioli, M. 2009. Distribution of living planktonic foraminifera in the Ross Sea and the Pacific sector of the Southern Ocean (Antarctica). Marine Micropaleontology, 73:37-48.

Berggren, W.A. 1972. Cenozoic biostratigraphy and paleobiogeography of the North Atlantic. p. 9651001. In Davies, T.A. (ed.), Initial Reports of the Deep Sea Drilling Project 7, Washington (U.S. Government Printing Office).

Berggren, W.A. 1973. The Pliocene time scale: Calibration of planktonic foraminiferal and calcareous nannoplankton zones. Nature, 243:391-397.

Berggren, W.A. 1977. Late Neogene planktonic foraminiferal biostratigraphy of the Rio Grande Rise (South Atlantic). Marine Micropaleontology, 2:265-313.

Berggren, W.A., Kent, D.V., Swisher, C.C.III, and Aubry, M.-P. 1995. A revised Cenozoic geochronology and chronostratigraphy, p. 129-212. In Berggren, W.A., Kent, D.V., Aubry, M.-P., and Hardenbol, J. (eds.), Geochronology Time Scales and Global Stratigraphic Correlation. SEPM Special Publication, no. 54. Tulsa, Oklahoma.

Bermúdez, P.J. 1937. Nuevas especies de foraminiferos del Eoceno de las cercanias de Guanajay, provincia Pinar del Rio, Cuba. Memorias de la Sociedad Cubana de Historia Natural, 11(4):237-248.

Bermúdez, P.J. 1952. Estudio sistematico de los foraminíferos rotaliformes. Bolétin de Geologia, Venezuela, 2(4):1-230. 
Bermúdez, P.J. 1961. Contribución al estudio de las Globigerinidea de la region Caribe-Antillana (PaleoceneReciente), Memoria del III Congreso Geológico Venezolano, Caracas, v. 3. Boletín de Geología, Publicación especial 3, 1960:1.119-1.393.

Bermúdez, P.J. and Fuenmayor, A.N. 1966. Consideraciones sobre los sedimentos del Mioceno medio al Reciente de las costas central y oriental de Venezuela. Segunda parte. Los foraminíferos bentónicos. Boletín de Geología, Venezuela, 7:413-611.

Bermúdez, P.J. and Seiglie, G.A. 1963. Estudio sistematico de los foraminiferos del Golfo de Cariaco. Boletin del Instituto Oceanográfico, Universidad de Oriente, Cumana, 2(2):1-267.

Berthelin, G. 1880. Mémoire sur les Foraminifères fossiles de l'Etage Albien de Moncley (Doubs). Mémoires de la Société géologique de France, ser.3, 1(5):1-85.

Blow, W.H. 1959. Age, correlation, and biostratigraphy of the upper Tocuyo (San Lorenzo) and Pozon formations, eastern Falcon, Venezuela. Bulletins of American Paleontology, 39:67-251.

Blow, W.H. 1965. Clavatorella, a new genus of the Globorotaliidae. Micropaleontology, 11:365-368.

Blow, W.H. 1969. Late Middle Eocene to Recent planktonic foraminiferal biostratigraphy. In Brönnimann, P., and Renz, H.H. (eds.), Proceedings of the First International Conference on Planktonic Microfossils, Geneva 1967, 1:199-422.

Blow, W.H. 1979. The Cainozoic Globigerinida. E.L. Brill, Leiden, 3 volumes.

Bolli, H.M. 1957. Planktonic foraminifera from the Oligocene-Miocene Cipero and Lengua formations of Trinidad, B.W.I. United States National Museum Bulletin, 215:97-123.

Bolli, H.M. and Bermudez, P.J. 1965. Zonation based on planktonic foraminifera of Middle Miocene to Pliocene warm-water sediments. Bulletin Informativo, Asociacion Venezolana de Geologia, Mineria y Petroleo, 8:119-149.

Bolli, H.M. and Bermudez, P.J. 1978. A neotype for Globorotalia margaritae Bolli and Bermudez. Journal of Foraminiferal Research, 8:138-142.

Bolli, H.M., Loeblich, A.R. Jr., and Tappan, H. 1957. Planktonic foraminiferal families Hantkeninidae, Orbulinidae, Globorotaliidae, and Globotruncanidae. United States National Museum Bulletin, 215:3-50.

Bolli, H.M. and Saunders, J.B. 1985. Oligocene to Holocene low latitude planktic foraminifera, p. 155-262. In Bolli, H.M., Saunders, J.B., and Perch-Nielsen, K. (eds.), Plankton stratigraphy. Cambridge University Press, Cambridge.

Boltovskoy, E. and Guissani de Kan, G. 1981. Cinco nuevos taxones en Orden Foraminiferida. Comunicaciones des Museo Argentino de Ciencias Naturales "Bernardino Rivadavia" e Instituto Nacional de Investigación de las Ciencias Naturales, Hydrobiologia, 2(5):43-51.
Boomgaart, L. 1949. Smaller Foraminifera from Bodjonegoro (Java). Smit and Dontje, Sappemeer.

Bornemann, J.G. 1855. Die mikroskopische Fauna des Septarienthones von Hermsdorf bei Berlin. Zeitschrift der deutschen geologischen Gesellschaft, 7:307371.

Bradshow, J.S. 1959. Ecology of living planktonic Foraminifera in the North and equatorial Pacific Ocean. Contributions from the Cushman Foundation for Foraminiferal Research, 10:25-64.

Brady, H.B. 1877. Supplementary note on the foraminifera of the Chalk(?) of the New Britain group. Geological Magazine, new series, 4:534-536.

Brady, H.B. 1878. On the reticularian and radiolarian Rhizopoda (Foraminifera and Polycystina) of the North-Polar Expeditions of 1875-76. The Annals and Magazine of Natural History, Fifth series, 6:425-550.

Brady, H.B. 1879. Notes on some of the reticularian Rhizopoda of the Challenger Expedition. Part I. Quarterly Journal of Microscopical Science, new series, 19:20-26.

Brady, H.B. 1881. Notes on some of the reticularian Rhizopoda of the Challenger Expedition. Part III. 1. Classification. 2. Further notes on new species. 3. Note on Biloculina mud. Quarterly Journal of Microscopical Science, new series, 21:31-71.

Brady, H.B. 1882. Report on the Foraminifera, p. 708719. In Tizard, T.H. and Murrey, J. (eds.), Exploration of the Faroe Channel, during the summer of 1880, in H.M.'s hired ship 'Knight Errant', Proceedings of the Royal Society of Edinburgh, 11.

Brady, H.B. 1884. Report on the foraminifera dredged by H.M.S. Challenger, during the years 1873-1876. Report on the Scientific Results of the Voyage of the Challenger during the years 1873-1876, 9 (Zoology):1-814.

Brady, H.B., Parker, W.K., and Jones, T. R. 1871. A monograph of the genus Polymorphina. Transactions of the Linnean Society of London, 27 (1870), part 2:197-253.

Brönnimann, P. 1951a. Globigerinita naparimaensis $\mathrm{n}$. gen., n. sp. from the Miocene of Trinidad, B.W.I. Contributions from the Cushman Foundation for Foraminiferal Research, 2:16-18.

Brönnimann, P. 1951b. The genus Orbulina d'Orbigny in the Oligo-Miocene of Trinidad, B.W.I. Contributions from the Cushman Foundation for Foraminiferal Research, 2:132-138.

Brönnimann, P. and Resig, J. 1971. A Neogene globigerinacean biochronologic time-scale of the southwestern Pacific, p. 1235-1469. In Winterer, E.L., Riedel, W.R., Brönnimann, P., Gealy, E.L., Heath, G.R., Kroenke[JENNIFER: need initials?], Martini, L.E., Moberly, R. Jr., Resig, J., and Worsley, T. (eds.), Initial Reports of the Deep Sea Drilling Project 7.

Brotzen, F. 1936. Foraminiferen aus dem Schwedischen untersten Senon von Eriksdal in Schonen: Arsbok Sveriges Geologiska Undersökning, 30:1-206. 
Brotzen, F. 1940. Flintrännans och trindelrännans Geologi. Arsbok Sveriges Geologiska Undersökning, 34(5):1-33.

Brotzen, F. 1942. Die Foraminiferengattung Gavelinella nov. gen. und die Systematik der Rotaliformes. Årsbok Sveriges Geologiska Undersökning, 36(8):1-60.

Brotzen, F. 1948. The Swedish Paleocene and its foraminiferal fauna. Årsbok Sveriges Geologiska Undersökning, 42(2):1-140.

Brünnich, M.Th. 1772. Zoologiae Fundamenta. Grunde i Dyrelaren, Hafniae et Lipsiae.

Carpenter, W.B., Parker, W.K., and Jones, T.R. 1862. Introduction to the study of foraminifera. Ray Society, London.

Chaisson, W.P. and D'Hondt, S.L. 2000. Neogene planktonic foraminifer biostratigraphy at Site 999, western Caribbean Sea, p. 19-56. In Leckie, R.M., Sigurdsson, H., Acton, G.D., and Draper, G. (eds.), Proceedings of the Ocean Drilling Program, Scientific Results, 165: College Station, TX (Ocean Drilling Program).

Chapman, F. 1907. Recent Foraminifera of Victoria: some littoral gatherings. Journal Quekett Microscopical Club, Series 2, 10:117-146.

Chapman, F. 1941. Report on foraminiferal soundings and dredgings of the F. I. S. "Endeavour" along the continental shelf of the south-east coast of Australia Transactions of the Royal Society of South Australia, 65:145-211.

Chapman, F. and Parr, W.J. 1935. Foraminifera and Ostracoda from soundings made by the trawler "Bonthorpe" in the Great Australian Bight. Journal of the Royal Society of Western Australia, 21(19341935):1-6.

Chapman, F. and Parr, W.J. 1937. Foraminifera. Australasian Antarctic Expedition, 1911-14 under the leadership of Sir Douglas Mawson. Scientific Reports, Series C, Zoology and Botany, 1(2):1-190.

Chapman, F., Parr, W.J., and Collins, A.C. 1934. Tertiary foraminifera of Victoria, Australia-The Balcombian deposits of Port Phillip, Part III. Journal of the Linnaean Society of London, Zoology, 38:553-577.

Cicha, I., Rögl, F., Pupp, C., and Streiniger, F.F. 1998. Oliogocene-Miocene foraminifera of the Central Paratethys. Senckenbergische Naturforschende Gesellschaft, 549:1-325.

Cifelli, R. 1961. Globigerina incompta, a new species of pelagic foraminifera from the North Atlantic. Contributions from Cushman Foundation for Foraminifera Research, 12:83-86.

Cifelli, R. 1973. Observations on Globigerina pachyderma (Ehrenberg) and Globigerina incompta Cifelli from the North Atlantic. Journal of Foraminiferal Research, 3:157-166.

Cifelli, R. 1982. Textural observations on some living species of planktonic foraminifera. Smithsonian Contributions to Paleobiology, 45:1-45. Smithsonian Institution Press, Washington DC.
Cifelli, R. 1990. Foraminiferal classification from d'Orbigny to Galloway. Cushman Foundation Special Publication, 27:1-88.

Cita, M.B., Premoli-Silva, F., and Rossi, R. 1965. Foraminiferi planctonici del Tortoniano-tipo. Rivista Italiana di Paleontologia e Stratigrafia, 71:217-308.

Collins, A.C. 1958. Foraminifera. British Museum (Natural History) Great Barrier Reef Expedition 1928-29, Scientific Reports, 6(6):335-437.

Costa, O.G. 1856. Paleontologia del regno di Napoli, Parte II. Atti dell' Accademia Pontaniana, Napoli, 7:113-378.

Costa, O.G. 1857. Foraminiferi fossili delle marne Terziarie di Messina. Memorie della Reale Accademia Scienza, Napoli, 2:127-147, 367-373.

Cushman, J.A. 1910. New arenaceous foraminifera from the Philippines. Proceedings of the United States National Museum, 36:437-424.

Cushman, J.A. 1911. A monograph of the foraminifera of the North Pacific Ocean. Part II. Textulariidae. United States National Museum Bulletin, 71(2):1-108.

Cushman, J.A. 1913. A monograph of the foraminifera of the North Pacific Ocean. Part III. Lageniidae. United States National Museum Bulletin, 71(3):1-125.

Cushman, J.A. 1917. New species and varieties of foraminifera from the Philippines and adjacent waters. Proceedings of the United States National Museum, 51(2172):651-662.

Cushman, J.A. 1918. Some Pliocene and Miocene foraminifera of the coastal plain of the United States. United States Geological Survey Bulletin, 676:1-100.

Cushman, J.A. 1921. Foraminifera of the Philippine and adjacent seas. United States National Museum Bulletin, 100(4):1-608.

Cushman, J.A. 1922a. Shallow-water foraminifera of the Tortugas Region. Publications of the Carnegie Institution of Washington, no. 311, Department of Marine Biology, 17:1-85.

Cushman, J.A. 1922b. The Foraminifera of the Atlantic Ocean. Part 3. Textulariidae. United States National Museum Bulletin, 104(3):1-143.

Cushman, J.A. 1922c. The foraminifera of the Byram Calcareous Marl at Byram, Mississippi. United States Geological Survey, Professional Paper, 129-E:87105.

Cushman, J.A. 1923. The foraminifera of the Atlantic Ocean. Part 4. Lagenidae. United States National Museum Bulletin, 104(4):1-228.

Cushman, J.A. 1924. Samoan foraminifera. Publications of the Carnegie Institution of Washington, no. 342, Department of Marine Biology Papers, 21:1-75.

Cushman, J.A. 1925. Notes on the genus Cassidulina. Contributions from the Cushman Laboratory for Foraminiferal Research, 1:51-60.

Cushman, J.A. 1926a. Recent foraminifera from Porto Rico. Publications of the Carnegie Institution of Washington, no. 344, Department of Marine Biology Papers, 23:73-84. 
Cushman, J.A. 1926b. Foraminifera of the typical Monterey of California. Contributions from the Cushman Laboratory for Foraminiferal Research, 2:53-69.

Cushman, J.A. 1927a. An outline of a re-classification of the foraminifera. Contributions from the Cushman Laboratory for Foraminiferal Research, 3:1-105.

Cushman, J.A. 1927b. Foraminifera of the genus Siphonina and related genera. Proceedings of the United States National Museum, 72(2716, art. 20):115.

Cushman, J.A. 1927c. Recent foraminifera from off the West coast of America. Bulletin of the Scripps Institution of Oceanography, Technical series, 1 (10):119188.

Cushman, J.A. 1930. The foraminifera of the Atlantic Ocean, Part 7. Nonionidae, Camerinidae, Peneroplidae and Alveolinellidae. United States National Museum Bulletin, 104(7):1-79.

Cushman, J.A. 1932. The foraminifera of the tropical Pacific collections of the "Albatross," 1899-1900. Part 1. Astrorhizidae and Trochamminidae. United States National Museum Bulletin, 161:1-88.

Cushman, J.A. 1933a. Some new foraminiferal genera. Contributions from the Cushman Laboratory of Foraminiferal Research, 9:32-38.

Cushman, J.A. 1933b. Some new Recent foraminifera from the tropical Pacific. Contributions from the Cushman Laboratory of Foraminiferal Research, 9:77-95.

Cushman, J.A. 1933c. The foraminifera of the tropical Pacific collections of the "Albatross," 1899-1900. Part 2. Lagenidae and Alveolinellidae. United States National Museum Bulletin, 161:1-79.

Cushman, J.A. 1934. Smaller foraminifera from Vitilevu, Fiji. Geology of Vitilevu, Fuji, Bernice P. Bishop Museum Bulletin, 119:102-141.

Cushman, J.A. 1935. Fourteen new species of Foraminifera. Smithsonian Miscellaneous Collections, 91(21):1-9.

Cushman, J.A. 1936. New genera and species of the families Verneuilinidae and Valvulinidae and of the subfamily Virgulininae. Cushman Laboratory for Foraminiferal Research Special Publications, 6:1-71.

Cushman, J.A. 1937a. A monograph of foraminiferal family Verneuilinidae. Cushman Laboratory for Foraminiferal Research Special Publications, 7:1-157.

Cushman, J.A. 1937b. A monograph of foraminiferal family Valvulinidae. Cushman Laboratory for Foraminiferal Research Special Publications, 8:1-210.

Cushman, J.A. 1942. The foraminifera of the tropical Pacific collections of the "Albatross," 1899-1900. Part 3. Heterohelicidae and Buliminidae. United States National Museum Bulletin, 161:1-67.

Cushman, J.A. 1945. The species of the subfamily Reussellinae of the foraminiferal family Buliminidae. Contributions from the Cushman Laboratory for Foraminiferal Research, 21:23-54.
Cushman, J.A. and Adams, B.C. 1935. New late Tertiary Bolivinas from California. Contributions from the Cushman Laboratory for Foraminiferal Research, 11:16-20.

Cushman, J.A. and Bermúdez, P.J. 1949. Some Cuban species of Globorotalia. Contributions from the Cushman Laboratory for Foraminiferal Research, 25:2645.

Cushman, J.A. and Brönnimann, P. 1948. Some new genera and species of foraminifera from brackish water of Trinidad. Contributions from the Cushman Laboratory for Foraminiferal Research, 24:15-21.

Cushman, J.A. and Edwards, P.G. 1937. Astrononion a new genus of the foraminifera, and its species. Contributions from the Cushman Laboratory for Foraminiferal Research, 13:29-36.

Cushman, J.A. and Edwards, P.G. 1938. Notes on the Oligocene species of Uvigerina and Angulogerina. Contributions from the Cushman Laboratory for Foraminiferal Research, 14:74-94.

Cushman, J.A. and Jarvis, P.W. 1929. New foraminifera from Trinidad. Contributions from the Cushman Laboratory for Foraminiferal Research, 5:6-17.

Cushman, J.A. and Jarvis, P.W. 1930. Miocene foraminifera from Buff Bay, Jamaica. Journal of Paleontology, 4(4):353-368.

Cushman, J.A. and Jarvis, P.W. 1936. Three new Foraminifera from the Miocene Bowden Marl, of Jamaica. Contributions from the Cushman Laboratory for Foraminiferal Research, 15:1-14.

Cushman, J.A. and Martin, L.T. 1935. A new genus of foraminifera, Discorbinella, from Monterey Bay, California. Contributions from the Cushman Laboratory for Foraminiferal Research, 11:89-90.

Cushman, J.A. and McCulloch, I. 1942. Some Virgulininae in the collections of the Allan Hancock Foundation. Allan Hancock Pacific Expeditions, 6(4):179230.

Cushman, J.A. and McCulloch, I. 1948. The species of Bulimina and related genera in the collections of the Allan Hancock Foundation. Allan Hancock Pacific Expeditions, 6(5):211-294.

Cushman, J.A. and Ozawa, Y. 1928. An outline of a revision of the Polymorphinidae. Contributions from the Cushman Laboratory for Foraminiferal Research, 4:13-21.

Cushman, J.A. and Parker, F.L. 1947. Bulimina and related foraminiferal genera. United States Geological Survey Professional Paper, 210-D:55-176.

Cushman, J.A., Todd, R., and Post, R.J. 1954. Recent foraminifera of the Marshall Islands, Bikini and nearby atolls, Part 2, oceanography (biologic). United States Geological Survey, Professional Paper, 260$\mathrm{H}: 319-384$.

Cushman, J.A. and Valentine, W.W. 1930. Shallow-water foraminifera from the Channel Islands of southern California. Contributions from the Department of Geology of Stanford University, 1(1):5-51. 
Cushman, J.A. and Wichenden, R.T.D. 1929. Recent foraminifera from off Juan Fernandez Islands. Proceedings of the United States National Museum, 75(2780):1-16.

Cžjžek, J. 1848. Beitrag zur Kenntniss der fossilen Foraminiferen des Wiener Beckens. Haidinger's Naturwissenschaftliche Abhandlungen, Wien, 2(1):137150.

Cžjžek, J. 1849. Über zwei neue Arten von Foraminiferen aus dem Tegel von Baden und Möllersdorf. Bericht über die Mittheilungen der Freunde der Naturwissenschaften in Wien, 5:50-56.

Darling, K.F., Kucera, M., and Wade, C.M. 2007. Global molecular phylogeography reveals persistent Arctic circumpolar isolation in a marine planktonic protist. Proceedings of the National Academy of Sciences of the United States of America, 104:5002-5007.

Darling, K.F., Kucera, M., Kroon, D., and Wade, C.M. 2006. A resolution for the coiling direction paradox in Neogloboquadrina pachyderma. Paleoceanography, 21, PA2011, doi:10.1029/2005PA001189.

Darling, K.F., Wade, C.M., Kroon, D., and Leigh Brown, A.J. 1997. Planktic foraminiferal molecular evolution and their polyphyletic origins from benthic taxa. Marine Micropaleontology, 30:251-266.

Dawson, J.W. 1860. Notice of Tertiary fossils from Labrador, Maine, etc., and remarks on the climate of Canada, in the newer Pliocene or Pleistocene period. Canadian Naturalist and Geologist, and Proceedings of the Natural History Society of Montreal, 5:188-200.

de Blainville, H.M. Ducrotay 1826. Dictionnaire des Sciences Naturelles, pin-plo, vol. 41, F.G. Levrault, Paris.

Defrance, J.L.M. 1824. Dictionnaire des Sciences Naturelles, vol. 32, F. G. Levrault, Strasbourg.

de Klasz, I., Kroon, D., and van Hinte, J.E. 1989. Notes on the Foraminiferal Genera Laterostomella De Klasz and Rérat and Streptochilus Brönnimann and Resig. Journal of Micropalaeontology, 8:215-225.

Delage, Y. and Hérouard, E. 1896. Traité de Zoologie Concrète, vol. 1, La Cellule et les Protozoaires. Schleicher Frères, Paris.

de Montfort, P.D. 1808. Conchyliologie Systématique, et Classification Méthodique des Coquilles 1. F. Schoell, Paris.

Dervieux, E. 1894. Le Nodosarie terziarie del Piemonte. Bolletino della Societá Geologica Italiana, 12:591626.

Doan, D.B., Paseur, J.E., and Fosberg, F.R. 1960. Military Geology of the Miyako Archipelago, RyūkyūRetto. Intelligence Division Office of the Engineer Headquarters, Unites States Army Pacific with personnel of the United States Geological Survey.

d'Orbigny, A. 1826. Tableau méthodique de la classe des Céphalopodes. Annales des Sciences Naturelles, 7:96-314.
d'Orbigny, A. 1839a. Foraminifères. In Ramon de la Sagra. Histoire physique, politique et naturelle de l'île de Cuba. p. 1-224. Arthus Bertrand, Paris.

d'Orbigny, A. 1839b. Foraminifères. p. 119-146. In P. Barker-Webb and Berthelot, S., Historie Naturelle des Îles Canaries, vol. 2, pt. 2, Zoologie. Bethune, Paris.

d'Orbigny, A. 1839c. Voyage dans l'Amérique méridionale, Foraminifères, V(5):1-86. C.P. Bertrand, Paris and Strasbourg.

d'Orbigny, A. 1846. Foraminifères fossiles du Bassin Tertiaire de Vienne (Autriche). Gide et Compe , Paris.

Domitsu, H. and Oda, M. 2005. Japan Sea planktic foraminifera in surface sediments: geographical distribution and relationships to surface water mass. Paleontological Research, 9:255-270.

Domitsu, H. and Oda, M. 2006. Linkages between surface and deep circulations in the southern Japan Sea during the last 27,000 years: Evidence from planktic foraminiferal assemblages and stable isotope records. Marine Micropaleontology, 61:155-170.

Domitsu, H. and Oda, M. 2008. Pleistocene planktic foraminiferal events in the Northwest Pacific near Japan. The Open Paleontology Journal, 1:1-6.

Drooger, C.W. 1953. Miocene and Pliocene foraminifera from Orangestad, Aruba (Netherland Antilles). Contributions from the Cushman Foundation for Foraminiferal Research, 4:115-147.

Eade, J.V. 1967. New Zealand Recent foraminifera of the families Islandiellidae and Cassidulinidae. New Zealand Journal of Marine and Freshwater Research, 1:421-454.

Eames, F.E., Banner, F.T., Blow, W.H., and Clarke, W.J. 1962. Fundamentals of Mid-Tertiary Stratigraphical Correlation. Cambridge University Press, Cambridge.

Earland, A. 1934. Foraminifera, part III. The Falklands sector of the Antarctic (excluding South Georgia). Discovery Reports, 10:1-208.

Egger, J.G. 1893. Foraminiferen aus Meeresgrundproben gelothet von 1874 bis 1876 von S. M. Sch. Gazelle. Abhandlungen der Bayerischen Akademie der Wissenschaften München, Mathematisch-Physikalische Klasse, 18(2):193-458.

Eguchi, N.O., Ujiié, H., and Kawahata, H. 2003. Seasonal variations in planktonic foraminifera at three sediment traps in the Subarctic, Transition and Subtropical zones of the central North Pacific Ocean. Marine Micropaleontology, 48:149-163.

Ehrenberg, C.G. 1838. Über dem blossen Auge unsichtbare Kalkthierchen und Kieselthierchen als Hauptbestandtheile der Kreidegebirge. Bericht über die $z u$ Bekanntmachung geeigneten Verhandlungen der Königlichen Preussischen Alademie der Wissenschaften zu Berlin, 1838:192-200. 
Ehrenberg, C.G. 1839. Über die Bildung der Kreidefelsen und des Kreidemergels durch unsichtbare Organismen. Physikalische Abhandlungen der Königlichen Akademie der Wissenschaften zu Berlin, 1838 [1840: separate 1839]:59-147.

Ehrenberg, C.G. 1861. Elemente des tiefen Meeresgrundes im Mexikanischen Golfstrome bei Florida; über die Tiefgrund-Verhältnisse des Oceans am Eingange der Davisstrasse und bei Island. Monatsbericht der Königlichen Preussischen Akademie der Wissenschaften zu Berlin 1861:275-315.

Ehrenberg, C.G. 1873. Mikrogeologische Studien über das kleinste Leben der Meeres-Tiefgründe aller Zonen und dessen geologischen Einfuss. Physikalische Abhandrlungen der Königlichen Akademie der Wissenschaften zu Berlin, 1872:131-397.

Eimer, G.H.T. and Fickert, C., 1899. Die Artbildung und Verwandtschaft bei den Foraminiferen. Entwurf einer natürlichen Eimtheilung derselben. Zeitschrift für Wissenschaftliche Zoologie, 65:599-708.

El-Naggar, Z.R. 1971. On the classification, evolution and stratigraphical distribution of the Globigerinacea. Farinacci, A. (ed.), Proceedings of the II Planktonic Conference, Roma, 1970, vol. 1: 421-476. Edizioni Tecnoscienza, Rome.

Eynaud, F., Cronin, T.M., Smith, S.A., Zaragosi, S., Mavel, J., Mary, Y., Mas, V., and Pujol, C. 2009. Morphological variability of the planktonic foraminifer Neogloboquadrina pachyderma from ACEX cores: Implications for Late Pleistocene circulation in Arctic Ocean. Micropaleontology, 55:101-116.

Farías, J.R. 1977. Murrayinella: taxa nuevo para la ciencia de foraminíferos del Reciente de Agua Somera. Revista Española de Micropaleontología, 9:343-345.

Finlay, H.J. 1939. New Zealand Foraminifera: Key species in stratigraphy-No. 1. Transactions of the Royal Society of New Zealand, 68:504-543.

Finlay, H.J. 1947. New Zealand Foraminifera: Key species in stratigraphy-No. 5. New Zealand Journal of Science and Technology, 28(5):259-292.

Fleming, J. 1828. A History of British Animals, Exhibiting the Descriptive Characters and Systematic Arrangement of the Genera and Species of Quadrupeds, Birds, Fishes, Mollusca and Radiata of the United Kingdom. Bell \& Bradfute, Edinburgh.

Fornasini, C. 1883. Nota preliminare sui foraminiferi della marna pliocenica del Ponticello di Savena nel Bolognese. Bollettino della Società Geologica Italiana, 2:176-190.

Fornasini, C. 1898. Le Globigerine fossili d'Italia. Palaeontographia Italica, 4:203-218.

Fornasini, C. 1906. Illustrazione di specie orgignyane di "Rotalidi" institute nel 1826. Memorie della R. Accademie della Scienze dell'lstituto di Bologna, Scienze Naturali, serie 6, 3:61-70.

Franzenau, A. 1884. Heterolepa egy uj genus a Foraminiferák rendjében. Természetrajzi Füzetek, Budapest, 8:181-184, 214-217.
Fujita, Y. and Ito, S. 1957. A study of foraminiferal assemblages from the Miocene formation, Date district, Fukushima Prefecture, Japan. The Journal of the Geological Society of Japan. 63:497-513. (In Japanese with abstract and taxonomic notes in English)

Fujita, K., Nishi, H., and Saito, T. 1999. Seasonality of living epiphytic foraminifera from seagrass beds in near shore zones of the Ryukyu Islands, Japan. Fossils (The Palaeontological Society of Japan), 66:16-33. (In Japanese with English abstract)

Furukawa, H. 1985. Miyakojima. p. 145-153. In Kizaki, K. (ed.), Geology of the Ryukyu Island Arc. Okinawa Times Ltd., Naha. (In Japanese)

Galloway, J.J. 1933. A Manual of Foraminifera. Principia Press, Bloomington.

Galloway, J.J. and Wissler, S.G. 1927a. Pleistocene foraminifera from the Lomita Quarry, Palos Verdes Hills, California. Journal of Paleontology, 1:35-87.

Galloway, J.J. and Wissler, S.G. 1927b. Correction of names of foraminifera. Journal of Paleontology, $1: 193$.

Giannelli, L. and Salvatorini, G. 1976. Due nuove specie di foraminiferi planctonici del Miocene. Bullettino della Società Paleontologica Italiana, 15:167-173.

Gibbard, P.L., Head, M.J., and Walker, M.J.C. 2010. Formal ratification of the Quaternary System/Period and the Pleistocene Series/Epoch with a base at 2.58 Ma. Journal of Quaternary Science, 25:96-102.

Glaessner, M.F. 1937. Die Entfaltung der Foraminiferen familie Buliminidae. Problemy Paleontologii, Paleontologischeskaya Laboratoriya Moskovskogo Gosudarstvennogo Universiteta, 2-3:411-422.

Gmelin, J.F. 1791. Caroli a Linné, systema naturae, 1:3021-3910. Lipsiae.

Goës, A., 1896. The Foraminifera. Reports on the dredging operations off the West Coast of Central America to the Galapagos, to the West coast of Mexico, and in the Gulf of California, in charge of Alexander Agassiz, carried on by the U.S. Fish Commission Steamer "Albatross," during 1891. Bulletin of the Museum of Comparative Zoölogy at Harbard, 29:1-103.

Gonzáles-Donoso, J.M. 1969. Données nouvelles sur la texture et la structure du test de quelques foraminifères du Bassin de Grenade (Espagne). Revue de Micropaléontologie, 12:3-8.

Gonzáles-Donoso, J.M. and Linares, D. 1970. Datos sobre los foraminíferos del Tortonense de Alcalá la Real (Jaén). Revista Española de Micropaleontología, 2:235-242.

Gradstein, F.M., Ogg, J.G., and Smith, A.G. (eds.), 2004. Geologic Time Scale 2004. Cambridge University Press, Cambridge, U.K.

Gradstein, F. M., Ogg, J.G., Schmitz, M.D., and Ogg, G.M. (eds.), 2012. The Geologic Time Scale 2012. 2 volumes, Elsevier.

Graham, J.J. and Militante, P.J. 1959. Recent foraminifera from the Puerto Galera area, northern Mindoro, Philippines. Stanford University Publications, Geological Sciences, 6(2), Stanford, California. 
Griffith, J.W. and Henfrey, 1875. The Micrographic Dictionary, vol. 1, 3rd ed. John Van Voorst, London.

Gronovius, L.T. 1781. Zoophylacii Gronoviani, 3:241380, Theodorus Haak et Soc., Leyden.

Gudina, V.I. and Saidova, Kh M. 1967. Novyy rod Alabamminoides (Foraminifera) I ego vidy. In Fursenko, A.V. (ed.), Foraminifery Mezozoya I Kaynozoya zapadnoy Sibiri, Taymyra I dal'nego Vostoka. Instituta Geologii I Geofiziki, Akademiya Nauk SSSR, Sibirskoe Otdelenie. Nauka, Moscow, p. 97-102. (In Russian)

Gümbel, C.W. 1868. Beiträge zur Foraminiferenfauna der nordalpinen, älteren Eocängebilde oder der Kressenberger Nummulitenschichten. Bayerische Akademie der Wissenschaften zur Berlin, Abhandlungen, II (Mathematische-Physikalische Klasse), 10:579-730.

Hada, Y. 1931. Report of the biological survey of Mutsu Bay. 19, Notes on the Recent foraminifera from Mutsu Bay. Science Reports of the Tohoku University, Fourth Series (Biology), 6(1):45-148.

Haeckel, E. 1894. Systematische Phylogenie. Entwurf eines Natürlichen Systems der Organismen auf Grund ihrer Stammesgeschichte. Theil 1, Systematische Phylogenie der Protisten und Pflanzen. Georg Reimer, Berlin.

Haig, D.W. 1993. Buliminid foraminifera from inner neritic sand and mud facies of the Papuan Lagoon, New Guinea. Journal of Foraminiferal Research, 23:162179.

Haman, D. 1984. Saidovina, new name for Loxostomina Saidova, 1975 (non Sellier de Civrieux, 1968) and the status of Loxostomella Saidova, 1975 (Foraminiferida). Proceedings of the Biological Society of Washington. 97:419.

Hanagata, S. 2004. Pliocene-Pleistocene planktonic foraminiferal biostratigraphy in the Miyagijima and adjacent islands, off Katsuren Peninsula, east of Okinawa-jima, Japan. Bulletin of the Mizunami Fossil Museum, 31:37-48. (In Japanese with English abstract)

Hansen, H.J. and Rögl, F. 1980. On Anomalina punctulata d'Orbigny. Journal of Foraminiferal Research, 10:153-155.

Hanzawa, S. 1935. Topography and geology of the Riukiu Islands. Science reports of the Tohoku Imperial University, second series (Geology), 17:1-61.

Hasegawa, S. 1991. Melonis uchioi, a new name for a homonym in the foraminiferal genus Melonis from the Pacific. Transactions and Proceedings of the Palaeontological Society of Japan, New Series, 164:1003.

Hasegawa, S. and Nomura, R. 1995. Redescription of Asano and Nakamura's (1937) cassidulinid species based on their primary types. Transactions and Proceedings of the Palaeontological Society of Japan, New Series, 178:89-104.
Hatta, A. and Ujiié, H. 1992a. Benthic foraminifera from coral seas between Ishigaki and Iriomote Islands, southern Ryukyu Island Arc, Northwestern Pacific. Part 1. Systematic descriptions of Textulariina and Miliolina. Bulletin of the College of Science, University of Ryukyus, 53:49-119.

Hatta, A. and Ujiié, H. 1992b. Benthic foraminifera from coral seas between Ishigaki and Iriomote Islands, southern Ryukyu Island Arc, Northwestern Pacific. Part 2. Systematic descriptions of Rotaliina. Bulletin of the College of Science, University of Ryukyus, 54:163-287.

Hayward, B.W. 2002. Late Pliocene to middle Pleistocene extinctions of deep-sea benthic foraminifera ("Stilostomella extinction") in the Southwest Pacific. Journal of Foraminiferal Research, 32:274-307.

Hayward, B.W., Carter, R., Grenfell, H.R., and Hayward, J.J. 2001. Depth distribution of Recent deep-sea benthic foraminifera east of New Zealand, and their potential for improving paleobathymetric assessments of Neogene microfaunas. New Zealand Journal of Geology and Geophysics, 44:555-587.

Hayward, B.W., Holzmann, M., Grenfell, H.R., Pawlowski, J., and Triggs, C.M. 2004. Morphological distinction of molecular types in Ammonia-towards a taxonomic revision of the world's most commonly misidentified foraminifera. Marine Micropaleontology, 50:237-271.

Hayward, B.W., Kawagata, S., Sabaa, A., Grenfell, H., van Kerckhoven, L., Johnson, K., and Thomas, E. 2012. The last global extinction (Mid-Pleistocene) of deep-sea benthic foraminifera (Chrysalogoniidae, Ellipsoidinidae, Glandulonodosariidae, Plectofrondiculariidae, Pleurostomellidae, Stilostomellidae), their Late Cretaceous-Cenozoic history and taxonomy. Cushman Foundation for Foraminiferal Research, Special Publication, no. 43.

Hermelin, J.O.R. 1989. Pliocene Benthic foraminifera from the Ontong-Java Plateau (western equatorial Pacific Ocean): Faunal response to changing paleoenvironments. Cushman Foundation for Foraminiferal Research, Special Publication, 26:1-143.

Heron-Allen, E. and Earland, A. 1913. Clare Island survey, Part 64, Foraminifera. Proceedings of the Royal Irish Academy, 31:1-188.

Hess, S. 1998. Distribution patterns of recent benthic foraminifera in the South China Sea. GeologischPaläontologisches Institut und Museum, ChristianAlbrechts-Universität Kiel, Deutschland, Berichte Reports, Nr. 91.

Hessland, I.R. 1943. Marine Schalenablagerungen Nord Bohusläns. Bulletin of the Geological Institutions of the University of Uppsala, 31:1-348.

Hofker, J. 1950. Wonderful animals of the sea: Foraminifera. Amsterdam Naturalist, 1(3):60-79.

Hofker, J. 1951. The Foraminifera of the Siboga Expedition. Part III. Siboga-Expeditie, Monographie IVa:1513. E.J. Brill, Leiden. 
Hofker, J. 1954. Über die Familie Epistomariidae (Foram.). Palaeontographica, 105A:166-206.

Hofker, J. 1956a. Tertiary foraminifera of coastal Ecuador: Part II, Additional notes on the Eocene species. Journal of Paleontology, 30:891-958.

Hofker, J. 1956b. Foraminifera dentata: Foraminifera of Santa Cruz and Thatch Island, Virginia Archipelago, West Indies. Spolia Zoologica Musei Hauniensis, 15:1-237.

Hofker, J. 1976. La famille Turborotalitidae. Revue de Micropaléontologie, 19:47-53.

Hofker, J. 1978. Biological results of the Snellius Expedition, XXX. The foraminifera collected in 1929 and 1930 in the eastern part of the Indonesian Archipelago. Zoologische Verhandelingen, Rijksmuseum van Natuurlijke Historie te Leiden, 161:1-69.

Hohenegger, J., Yordanova, E., Nakano, Y., and Tatzreiter F. 1999. Habitats of larger foraminifera on the upper reef slope of Sesoko Island, Okinawa, Japan. Marine Micropaleontology, 36:109-168.

Holbourn, A.E. and Hederson, A.S. 2002. Re-illustration and revised taxonomy for selected deep-sea benthic foraminifers. Palaeontologia Electrica 4(2):34pp., 628KB; http://palaeo-electronica.org/paleo/2001_2/ foram/issue2_01.htm

Hornibrook, N. de B. 1964. The foraminiferal genus Astrononion Cushman and Edwards. Micropaleontology, 10:333-338.

Huang B., Jian Z., and Wang P. 2007. Benthic foraminiferal fauna turnover at 2.1 Ma in the northern South China Sea. Chinese Science Bulletin, 52:839-843.

Huang, T. 1967. Late Tertiary planktonic foraminifera from southern Taiwan. Science Reports of the Tohoku University, second series (Geology), 38:165192.

Huang, T. 1968. Smaller foraminifera from Miyako-jima, Ryukyu. Science Reports of the Tohoku University, second series (Geology), 40:47-63.

Huang, T. 1986. Alloglobigerinoides, a new planktic foraminiferal genus. Petroleum Geology of Taiwan, 22:93-102.

Husezima, R. and Maruhasi, M. 1944. A new genus and thirteen new species of foraminifera from the coresample of Kashiwazaki oil field, Niigata-ken. Journal Sigenkagaku Kenkyusho, 1:391-400.

laccarino, S. 1985. Mediterranean Miocene and Pliocene planktic foraminifera, p. 283-314. In Bolli, H.M., Saunders, J.B., and Perch-Nielsen, K. (eds.), Plankton stratigraphy. Cambridge University Press, Cambridge.

Ibaraki, M. 1985. Neogene planktonic foraminiferal biostratigraphy of the Kakegawa area on the Pacific coast of central Japan. Reports of Faculty of Science, Shizuoka University, 20:39-173.

Ibaraki, M. and Tsuchi, R. 1975. Planktonic foraminifera from the upper part of the Neogene Shimajiri Group and the Chinen Sand, the Okinawa Island. Reports of Faculty of Science, Shizuoka University, 10:129-143.
Inoue, Y. 1989. Northwest Pacific foraminifera as paleoenvironmental indicators. Science Reports of the Institute of Geoscience, University of Tsukuba, Section B (Geological Sciences), 10:57-162.

Ishizaki, K. 1943. On the species of Ellipsonodosaria from Japan. Transactions of the Natural History Society of Taiwan, 33:678-689.

Ishizaki, K. 1944. New species of Neogene, Pleistocene and Recent foraminifera of Japanese Empire (2). Transactions of the Natural History Society of Taiwan, 34:98-104.

Ishizaki, K. 1948. Six new fossil species of Streblus from Eastern Asia. Acta Geologia Taiwanica, Science Report of National Taiwan University, First series, 2(1):55-66.

Iwasa, S. 1955. Biostratigraphy of the Isizawagawa Group in Honjô and its environs, Akita Prefecture. The Journal of the Geological Society of Japan, 61:118. (In Japanese with English abstract)

Iwasa, S. and Kikuchi, Y. 1954. Foraminifera from the Sugota Formation, Akita Prefecture, Japan. Transactions and Proceedings of the Palaeontological Society of Japan, New Series, 16:183-194.

Jenkins, D.G. 1985. Southern mid-latitude Paleocene to Holocene planktic foraminifera, p. 263-282. In Bolli, H.M., Saunders, J.B., and Perch-Nielsen, K. (eds.), Plankton stratigraphy, Cambridge University Press, Cambridge.

Jones, R.W. 1984. A revised classification of the unilocular Nodosariida and Buliminida (Foraminifera). Revista Española de Micropaleontologia, 16:91-160.

Jones, R.W. 1994. The Challenger Foraminifera. Oxford University Press and The Natural History Museum, London.

Kadar, D. 1975. Planktonic foraminifera from the lower part of the Sentolo Formation, central Java, Indonesia. Journal of Foraminiferal Research, 5:1-20.

Kadar, D. 1977. Upper Miocene planktonic foraminifera from Bali. Jourbach Geologische Bundesanster Sonderbund, 19(5):58-70.

Kadar, D. 1992. Rotaliid foraminifera from the Rembang zone area, north Central Java, Indonesia, p. 245256. In Ishizaki, K. and Saito, T. (eds.), Centenary of Japanese Micropaleontology. Terra Scientific Publishing Company, Tokyo.

Kaiho, K. 1992. Eocene to Quaternary benthic foraminifers and paleobathymetry of the Izu-Bonin Arc, Legs 125 and 126, p. 285-310. In Taylor, B., Fujioka, K., Janecek, T.R., and Langmuir, C. (eds.), Proceedings of the Ocean Drilling Program, Scientific Results, 126: College Station, TX (Ocean Drilling Program).

Kaiho, K. and Nishimura, A. 1992. Distribution of Holocene benthic foraminifers in the Izu-Bonin Arc. p. 311-320. In Taylor, B., Fujioka, K., Janecek, T.R., and Langmuir, C. (eds.), Proceedings of the Ocean Drilling Program, Scientific Results, 126: College Station, TX (Ocean Drilling Program). 
Kameyama, T. 1975. Smaller foraminiferas from Ogamijima, Ryukyu Islands. Earth Science; Journal of the Association for the Geological Collaboration in Japan, 29:275-279.

Kaminski, M.A., Aksu, A., Box, M., Hiscott, R.N., Filipescu, S., and Al-Salameen, M. 2002. Late Glacial to Holocene benthic foraminifera in the Marmara Sea: implications for Black Sea-Mediterranean Sea connections following the last deglaciation. Marine Geology, 190:165-202.

Kanmacher, F. 1798. Adam's Essays on the Microscope; the Second Edition, with Considerable Additions and Improvements. Dillon and Keating, London.

Karasawa, H. and Nobuhara, T. 2008. Decapoda and Isopoda (Crustacea) from the Pliocene Shimajiri Group in the Miyako-jima island, Ryukyus, Japan. Bulletin of the Mizunami Fossil Museum, 34:23-30.

Karrer, F. 1877. Geologie der Kaiser Franz-Josefs Hochquellen-Wasserleitung. Eine Studie in den Tertiär-Bildungen am Westrande des Alpinen Theil der Niederung von Wien. K. K. Geologischen Reichsanstalt, 9.

Karrer, F. 1868. Die Miocene Foraminiferenfauna von Kostej im Banat. Sitzungsberichte der Kaiserlichen Akademie der Wissenschaften Wien, Mathematischnaturwissenschaftliche Klasse, 58:121-193.

Karrer, F. 1878. Die Foraminiferen der Tertiären Thone von Luzon, p. 77-99. In Drasche, R. von (ed.), Fragmente zu einer Geologie der Insel Luzon (Philippinen). Verlag von Karl Gerold's Sohn, Wien.

Kawagata, S. 2001. Late Neogene benthic foraminifera from Kume-jima Island, central Ryukyu Islands, southwestern Japan. Science Reports of the Institute of Geoscience, University of Tsukuba, Section B (Geological Sciences), 22:61-123.

Kawagata, S. and Hatta, A. 2002. Internal test morphology of the genus Rectobolivina (Cushman, 1927) from the Late Cenozoic Miyazaki Group, southwestern Japan. Paleontological Research, 6:219-236.

Kawagata, S., Hayward, B.W., Grenfell, H.R., and Sabaa, A. 2005. Mid-Pleistocene extinction of deepsea foraminifera in the North Atlantic Gateway (ODP sites 980 and 982). Palaeogeography, Palaeoclimatology, Palaeoecology, 221:267-291.

Kawai, K., Uchio, T., Ueno, M., and Hozuki, M. 1950. Natural gas in the vicinity of Otaki, Chiba-ken. Journal of the Japanese Association for Petroleum Technology, 15:151-219. (In Japanese with English abstract)

Keller, G. 1978a. Late Neogene biostratigraphy and paleoceanography of DSDP Site 310 Central North Pacific and correlation with the Southwest Pacific. Marine Micropaleontology, 3:97-119.

Keller, G. 1978b. Morphologic variation of Neogloboquadrina pachyderma (Ehrenberg) in sediments of the marginal and Central Northeast Pacific Ocean and paleoclimatic interpretation. Journal of Foraminiferal Research, 8:208-224.
Keller, G. 1978c. Late Neogene planktonic foraminiferal biostratigraphy and paleoceanography of the northeastern Pacific: evidence from DSDP Sites 173 and 310 at the North Pacific Front. Journal of Foraminiferal Research, 8:332-349.

Kennett, J.P. 1966. The Globorotalia crassaformis bioseries in North Westland and Marlborough, New Zealand. Micropaleontology, 12:235-245.

Kennett, J.P. 1968. Latitudinal variation in Globigerina pachyderma (Ehrenberg) in surface sediments of the southwest Pacific Ocean. Micropaleontology, 14:305318.

Kennett, J.P. and Srinivasan, M.S. 1983. Neogene Planktonic Foraminifera -A Phylogenetic Atlas-. Hutchinson Ross Publishing Company, Stroudsburg.

Kim, J. 1999. Early Neogene biochemostratigraphy of Pohang Basin: a paleoceanographic response to the early opening of the Sea of Japan (East Sea). Marine Micropaleontology, 36:269-290.

Kim, J. and Kucera, M. 2000. Benthic foraminiferal record of environmental changes in the Yellow Sea (Hwanghae) during last 15,000 years. Quaternary Science Reviews 19:1067-1085.

Kisel'man, E.N. 1972. Verkhnemelovye I Paleotsenovye foraminifery novogo roda Spiroplectinella. Trudy Sibirskogo Nauchno-Issledovatel'skogo Instituta Geologii Geofiziki I Mineral'nogo Syr'ya (SIIGGIMS) Ministerstva Geologii I Okhrany Nedr SSSR, Novosibirsk, 146:134-140. (In Russian)

Koch, R. 1923. Die jungtertiäre Foraminiferenfauna von Kabu (Res. Surabaja, Java). Eclogae geologicae Helvetiae, 18(2):342-361.

Kučera, M. 1998. Biochronology of the mid-Pliocene Sphaeroidinella event. Marine Micropaleontology, 35:1-16.

Kučera, M. and Kennett, J.P. 2000. Biochronology and evolutionary implications of Late Neogene California margin planktonic foraminiferal events. Marine Micropaleontology, 40:67-81.

Kurihara, K. 1971. Foraminifera from the Hayama Group, Miura Peninsula. Transactions and Proceedings of the Palaeontological Society of Japan, New Series, 83:131-142.

Kuwano, Y. 1950. New species of foraminifera from the Pliocene formations of Tama Hills in the vicinity of Tokyo. The Journal of the Geological Society of Japan, 56:311-321.

Lalicker, C.G. and McCulloch, I. 1940. Some Textulariidae of the Pacific Ocean. Allan Hancock Pacific Expeditions, 6(2): 115-143.

Lamarck, J.B. 1804. Suite des mémoires sur les fossiles des environs de Paris. Annales Muséum National d'Histoire Naturelle, 5:179-188.

Lamarck, J.B. 1812. Extrait du cours de Zoologie du Muséum d'Historie Naturelle sur les animaux invertèbres. d'Hautel, Paris.

Lamarck, J.B. 1822. Histoire naturelle des animaux sans vertèbres, vol. 7. L'auteur, Paris. 
Larsen, A.R. 1977. A neotype of Amphistegina lessonii d'Orbigny, 1826. Journal of Foraminiferal Research, 7:273-277.

Le, J. and Shackleton, N.J. 1992. Carbonate dissolution fluctuations in the western equatorial Pacific during the late Quaternary. Paleoceanography, 7:21-42.

Le Calvez, Y. 1949. Révision des foraminifères Lutétiens du Bassin de Paris. II. Rotaliidae et familles affines. Mémoires du Service de la Carte Géologique Détaillée de la France:1-54.

Le Calvez, Y. 1974. Révision des foraminiféres de la collection d'Orbigny I. Foraminifères des Îles Canaries. Cahiers de Micropaléontologie, 1974(2):1-108.

LeRoy, L.W. 1939. Some small foraminifera, ostracoda, and otoliths from the Neogene ("Miocene") of the Rokan-Tapanoeli area, central Sumatora. Natuurkundig Tijdschrift voor Nederlandsch-Indië, 99:215-296.

LeRoy, L.W. 1941. Small foraminifera from the Late Tertiary of the Nederlands East Indies, Parts 1-3. Colorado School of Mines Quarterly, 36(1):1-132.

LeRoy, L.W. 1944. Miocene foraminifera from Sumatora and Java, Netherlands East Indies, Parts 1-2. Colorado School of Mines Quarterly, 39(3):1-113.

LeRoy, L.W. 1964. Smaller foraminifera from the Late Tertiary of southern Okinawa. United States Geological Survey Professional Paper, 454-F.

Li, Q., Zhong, G., and Tian, J. 2009. Stratigraphy and sea level changes. p. 75-170. In Wang, P. and Li, Q. (eds.), The South China Sea, Developments and Paleoenvironmental Research 13, Springer Science+Business Media B.V.

Li, B., Jian, Z., Li, Q., Tian, J., and Wang, P. 2005. Paleoceanography of the South China Sea since the middle Miocene: evidence from planktonic foraminifera. Marine Micropaleontology, 54:49-62.

Linnaeus, C. 1758. Systema Naturae. 10th ed., vol. 1, Holmiae (Stockholm): L. Salvii.

Lipps, J.H. 1965. Revision of the foraminiferal family Pseudoparrellidae Voloshinova. Tulane Studies in Geology, 3:117-147.

Loeblich, A.R., Jr. and Tappan, H. 1953. Studies of Arctic Foraminifera. Smithsonian Miscellaneous Collections, 121(7): 1-150.

Loeblich, A.R., Jr. and Tappan, H. 1957. The new planktonic foraminiferal genus Tinophodella, and an emendation of Globigerinita Brönnimann. Journal of the Washington Academy of Sciences, 47:112-116.

Loeblich, A.R., Jr. and Tappan, H. 1961. Suprageneric classification of the Rhizopodea. Journal of Paleontology, 35:245-330.

Loeblich, A.R., Jr. and Tappan, H. 1964. Sarcodina chiefly "Thecamoebians" and Foraminiferida. In Moore, R.C. (ed.), Treatise on Invertebrate Paleontology, Part C, Protista 2. Geological Society of America, Boulder and University of Kansas Press, Lawrence.

Loeblich, A.R., Jr. and Tappan, H. 1984. Suprageneric classification of the Foraminiferida (Protozoa). Micropaleontology, 30:1-70.
Loeblich, A.R., Jr. and Tappan, H. 1985. Some new and redefined genera and families of agglutinated foraminifera II. Journal of Foraminiferal Research, 15:175-217.

Loeblich, A.R., Jr. and Tappan, H. 1986. Some new and revised genera and families of hyaline calcareous Foraminiferida (Protozoa). Transactions of the American Microscopical Society, 105;239-265.

Loeblich, A.R., Jr. and Tappan, H. 1987. Foraminiferal Genera and their Classification. Van Nostrand Reinhod Company, New York, 2 volumes.

Loeblich, A.R., Jr. and Tappan, H. 1994. Foraminifera of the Sahul Shelf and Timor Sea. Cushman Foundation Special Publication, 31:1-661.

Lomnicki, J.R. 1901. Einige Bemerkungen zum Aufsatze: Die miocänen Foraminiferen in der Umgebung von Kolomea. Verhandlungen des naturforschenden Vereines Brünn, 39:15-18.

Łuczkowska, E. 1972. Miliolidae (Foraminiferida) from Miocene of Poland Part I. Revision of the classification. Acta Palaeontologica Polonica, 17:341-377.

Łuczkowska, E. 1974. Miliolidae (Foraminiferida) from Miocene of Poland Part II. Biostratigraphy, palaeoecology and systematics. Acta Palaeontologica Polonica, 19:3-176.

Makiyama, J. 1931. Stratigraphy of the Kakegawa Pliocene in Totomi. Kyoto Imperial University Collections Science Memoirs, Kyoto, Japan, series B, 7(1):1-54.

Malumián, N., Náñez, C., and Caramés, A. 1991. Unilocular foraminifera of reticular surface from Argentina. Micropaleontology, 37:393-406.

Martini, E. 1971. Standard Tertiary and Quaternary Calcareous Nannoplankton Zonation. p. 739-785. In Farinacci, A. (ed.), Proceedings of the II Planktonic Conference Roma , 1970, Tecnoscienza, Rome.

Mathews, R.D. 1945. Rectuvigerina, a new genus of foraminifera from a restudy of Siphogenerina. Journal of Paleontology, 19:588-606.

Matoba, Y. 1967. Younger Cenozoic foraminiferal assemblages from the Choshi district, Chiba Prefecture. Science Reports of the Tohoku University, second series (Geology), 38:221-263.

Matoba, Y. 1984. Paleoenvironment of the Sea of Japan, p. 409-414. In Benthos'83; Second International Symposium of Benthic Foraminifera (Pau April 1983).

Matsunaga, T. 1963, Benthonic smaller foraminifera from the oil fields of northern Japan. Science Reports of the Tohoku University, second series (Geology), 35:67-122.

McCulloch, I. 1977. Qualitative Observations on Recent Foraminiferal Tests with Emphasis on the Eastern Pacific: Parts I-III. University of Southern California, Los Angeles.

McCulloch, I. 1981. Qualitative Observations on Recent Foraminiferal Tests with Emphasis on the Eastern Pacific: Part IV. University of Southern California, Los Angeles. 
Mikhalevich, V.I. 1981. Parallelizm I konvergentsiya v evolyutsii skeketov foraminifer. Trudy Zoologischeskogo Instituta, Akademiya Nauk SSSR, 107:19-41. (In Russian)

Milker, Y. and Schmiedl, G. 2012. A taxonomic guide to modern benthic shelf foraminifera of the western Mediterranean Sea. Palaeontologia Electronica, 15:16A,134 pp.; palaeo-electronica.org/content/ 2012-issue-2-articles/223-taxonomy-foraminifera

Millett, F.W. 1898-1904. Report on the Recent Foraminifera of the Malay Archipelago contained in anchormud collected by Mr. A. Durrand, F. R. M. S. Parts IXVII. Journal of Royal Microscopical Society, 1898:258-269 (Part I), 499-513 (Part II), 607-614 (Part III); 1899:249-255 (Part IV), 357-365 (Part V), 557-564 (Part VI); 1900:6-13 (Part VII), 273-281 (Part VIII), 539-549 (Part IX); 1901:1-11 (Part X), 485-497 (Part XI), 619-628 (Part XII); 1902:509-528 (Part XIII); 1903:253-275 (Part XIV), 685-704 (Part XV); 1904, 489-506 (Part XVI), 597-609 (Part XVII).

Milne-Edwards, A. 1881. Compte rendu sommaire d'une exploration zoologique, faite dans le Méditerranée, à bord du navire de l'Etat "Le Travailleur." Compte Rendu Hebdomadaire des Séances de l'Académie des Sciences, Paris, 93:876-882.

Moncharmont Zei, M. and Sgarrella, F. 1978. Pytine parthenopeia n. gen. et n. sp. (Nodosariidae, Foraminiferida) del Golfo di Napoli. Bullettino della Società dei Naturalisti in Napoli, 86:1-7.

Montagu, G. 1803. Testacea Britannica, or Natural History of British Shells, Marine, Land and Fresh Water, Including the Most Minute. J. S. Hollis, Romsey, England.

Morkhoven, F.P.C.M., van, Berggren, W.A., and Edwards, A.S. 1986. Cenozoic cosmopolitan deepwater benthic foraminifera. Bulletin des Centres de Recherches Exploration-Production Elf-Aquitaine, 11.

Murata, S. 1951. On the paleo-ecological investigation of the fossil foraminiferal fauna in the Miyazaki Group, with description of new species. Bulletin of the Kyushu Institute of Technology, 1:91-104.

Murray, J. 2006. Ecology and Applications of Benthic Foraminifera. Cambridge University Press, Cambridge.

Nakagawa, H., Kitamura, N., Takayanagi, Y., Sakai, T., Oda, M., Asano, K., Niitsuma, N., Takayama, T., Matoba, Y., and Kitazato, H. 1976. Magnetostratigraphic correlation of Neogene and Pleistocene between the Japanese Island, Central Pacific, and Mediterranean Regions. Proceedings of the First International Congress of Pacific Neogene Stratigraphy:185-310.

Nakamori, T. 1982. Geology of Miyako Gunto, Ryukyu Islands, Japan. Contributions from the Institute of Geology and Paleontology, Tohoku University, 84:2339. (In Japanese with English abstract)
Nakamura, M. 1937. New species of fossil Foraminifera from the Byôritu Beds of the oil fields of northern Taiwan (Formosa), Japan. Japanese Journal of Geology and Geography, 14:133-142.

Nathan, S.A. and Leckie, R.M. 2009. Early history of the Western Pacific Warm Pool during the middle to late Miocene ( 13.2-5.8 Ma): Role of sea-level change and implications for equatorial circulation. Palaeogeography, Palaeoclimatology, Palaeoecology, 274: 140-159.

Natland, M.L. 1938. New species of foraminifera from off the West Coast of North America and from the later Tertiary of the Los Angeles Basin. University of California, Scripps Institution of Oceanography Bulletin, Technical Series, 4(5):137-164.

Natori, H. 1976. Planktonic foraminiferal biostratigraphy and datum planes in the Late Cenozoic sedimentary sequence in Okinawa-jima, Japan, p. 214-243. In Takayanagi, Y. and Saito, T. (eds.), Progress in micropaleontology, Micropaleontology Press, New York.

Neugeboren, J.L. 1856. Die Foraminiferen aus der Ordnung der Stichostegier von Ober-Lapugy in Siebenbürgen. Denkschriften der Kaiserlichen Akademie der Wissenschaften, Mathematisch-Naturwissenschaftliche Classe, 12:65-108.

Nicol, D. 1944. New West American species of the Foraminiferal genus Elphidium. Journal of Paleontology, 18:172-185.

Nomura, R. 1983a. Cassidulinidae (Foraminiferida) from the Uppermost Cenozoic of Japan (Part 1). Science Reports of the Tohoku University, second series (Geology), 53:1-101.

Nomura, R. 1983b. Cassidulinidae (Foraminiferida) from the Uppermost Cenozoic of Japan (Part 2). Science Reports of the Tohoku University, second series (Geology), 54:1-93.

Nomura, R. 1999. Miocene Cassidulinid Foraminifera from Japan. Special Papers, Palaeontological Society of Japan, no. 38.

Nomura, R. and Takayanagi, Y. 2000. The suprageneric classification of the foraminiferal genus Murrayinella ad a new species from Japan. Paleontological Research, 4:171-181.

Norris, R.D., Corfield, R.M., and Cartlidge, J.E. 1994. Evolutionary ecology of Globorotalia (Globoconella) (planktic foraminifera). Marine Micropaleontology, 23:121-145.

Nørvang, A. 1945. The Zoology of Iceland. Vol. II, Part 2: Foraminifera. Ejnar Munksgaard, København and Reykjavik.

Oda, M. 1978. Planktonic foraminiferal biostratigraphy of the Late Cenozoic sedimentary sequence, central Honshu, Japan. Science Reports of the Tohoku University, second series (Geology), 48:1-76. 
Oda, M. and Domitsu, H. 2009. Paleoceanographic significance of Neogloboquadrina pachyderma and Neogloboquadrina incompta. Fossils (The Palaeontological Society of Japan), 86: 6-11. (In Japanese with English abstract)

Ogasawara, K. and Masuda, K. 1983. Notes on the paleoenvironments based upon the Cenozoic molluscs in the Ryukyu Islands. The Memoirs of the Geological Society of Japan, 22:95-105.

Ohkushi, K., Thomas, E., and Kawahata. H. 2000. Abyssal benthic foraminifera from the northwestern Pacific (Shatsky Rise) during the last 298 kyr. Marine Micropaleontology, 38:119-147.

Ōki, K. 1989. Ecological analysis of benthonic foraminifera in Kagoshima Bay, South Kyūshū, Japan. South Pacific Study, 10:1-191.

Ōki, K. and Yamamoto, H. 1992. Notes on marine Quaternary sediments newly found in the west coastal area of the Satsuma Peninsula, Kyushu, Japan, with special references to the benthic foraminiferal assemblages, p. 189-205. In Ishizaki, K. and Saito, T. (eds.), Centenary of Japanese Micropaleontology. Terra Scientific Publishing Company, Tokyo.

Olsson, R.K. 1974. Pleistocene paleoceanography and Globigerina pachyderma (Ehrenberg) in site 36, DSDP, northeastern Pacific. Journal of Foraminiferal Research, 4:47-60.

Papp, A. and Schmid, M.E. 1985. The fossil foraminifera of the Tertiary basin of Vienna. Revision of the monograph by ALCIDE d'ORBIGNY (1846). Abhandlungen der Geologischen Bundesanstalt, 37.

Parker, F.L. 1962. Planktonic foraminiferal species in Pacific sediments. Micropaleontology, 8:219-254.

Parker, F.L. 1967. Late Tertiary biostratigraphy (planktonic foraminifera) of tropical Indo-Pacific deep-sea cores. Bulletins of American Paleontology, 52:115208.

Parker, W.K. and Jones, T.R. 1863. On the nomenclature of the foraminifera: Part VIII-Textularia. Annals and Magazine of Natural History, Third Series, 11:91-98.

Parker, W.K. and Jones, T.R. 1865. On some Foraminifera from the North Atlantic and Arctic Oceans, including Davis Straits and Baffin's Bay. Philosophical Transactions of the Royal Society of London, 155:325-441.

Parker, W.K., Jones, T.R., and Brady, H.B. 1865. On the nomenclature of the foraminifera, Part 12: the species enumerated by d'Orbigny in the "Annales des Sciences Naturelles," vol. 7, 1826. Annals and Magazine of Natural History, Third Series, 16:15-41.

Parr, W.J. 1932a. Victorian and South Australian shallow-water foraminifera. Part I. Proceedings of the Royal Society of Victoria, new series, 44:1-14.

Parr, W.J. 1932b. Victorian and South Australian shallow-water foraminifera. Part II. Proceedings of the Royal Society of Victoria, new series, 44:218-234.

Parr, W.J. 1942. New genera of foraminifera from the Tertiary of Victoria. Mining and Geological Journal, 2:361-363.
Parr, W.J. 1947. The lagenid foraminifera and their relationships. Proceedings of the Royal Society of Victoria, new series, 58:116-130.

Parr, W.J. 1950. Foraminifera. Reports of British and New Zealand Antarctic Research Expedition 19291931, Series B (Zoology and Botany), 5(6):233-392.

Patterson, R.T. 1985. Abditodentrix, a new foraminiferal genus in family Bolivinitidae. Journal of Foraminiferal Research, 15:138-140.

Patterson, R.T. 1987. Four new foraminiferal (Protozoa) genera from the Rio Grande Rise, southwest Atlantic Ocean. Transactions of the American Microscopical Society, 106:139-148.

Patterson, R.T. 2010. Moncharmontzeiana: New name for Pytine Moncharmont Zei and Sgarella, 1978 Non Fortey, 1975. Palaeontologia Electronica, 13, 10A; 2 pp.; http://palaeo-electronica.org/2010_2/223/index/ html.

Patterson, R.T. and Pettis, R.H. 1986. Galwayella, a new foraminiferal genus and new names for two foraminiferal homonyms. Journal of Foraminiferal Research, 16:74-75.

Patterson, R.T. and Richardson, R.P. 1987. A taxonomic revision of unilocular foraminifera. Journal of Foraminiferal Research, 17:212-216.

Patterson, R.T. and Richardson, R.P. 1988. Eight new genera of unilocular Foraminiferida, family Lagenidae. Transactions of the American Microscopical Society, 107:240-258.

Pawlowski, J., Holzmann, M., and Tyszka, J. 2013. New supraordinal classification of Foraminifera: Molecules meet morphology. Marine Micropaleontology, 100:110.

Phleger, F.B. 1952. Foraminifera distribution in some sediment samples from the Canadian and Greenland Arctic. Cushman Foundation for Foraminiferal Research, 3:80-89.

Phleger, F.B. and Parker, F.L. 1951. Ecology of foraminifera, northwest Gulf of Mexico. Part II. Foraminifera species. Memoirs of the Geological Society of America, 46(2):1-64.

Plummer, H.J. 1931. Gaudryinella, a new foraminiferal genus. American Midland Naturalist, 12:341-342.

Polski, W. 1959. Foraminiferal biofacies off the north Asiatic Coast. Journal of Paleontology, 33:569-587.

Poore, R.Z. and Berggren, W.A. 1975. The morphology and classification of Neogloboquadrina atlantica (Berggren). Journal of Foraminiferal Research, 5:7584.

Puri, H.S. 1954. Contribution to the study of the Miocene of the Florida panhandle. Bulletin of the Florida State Geological Survey, 36:1-345.

Ravelo, A.C. and Fairbanks, R.G. 1992. Oxygen isotopic composition of multiple species of planktonic foraminifera: Recorders of the modern photic zone temperature gradients. Paleoceanography, 7:815-832.

Reiss, Z. 1960. Structure of so-called Eponides and some other rotaliiform foraminifera. Bulletin of the Geological Survey of Israel, 29:1-28. 
Reiss, Z. 1963. Reclassification of perforate foraminifera. Bulletin of the Geological Survey of Israel, 35:1-111.

Resig, J.M. 1958. Ecology of foraminifera of the Santa Cruz Basin, California. Micropaleontology, 4:287308.

Reuss, A.E. 1850. Neues Foraminiferen aus den Schichten des österreichischen Tertiärbeckens. Denkschriften der Kaiserlichen Akademie der Wissenschaften, Mathematisch-Naturwissenschaftliche Classe, 1:365-390.

Reuss, A.E. 1851. Ueber die fossilen Foraminiferen und Entomostraceen der Septarienthone der Umgegend von Berlin. Zeitschrift der deutschen geologischen Gesellschaft, 3:49-92.

Reuss, A.E. 1860. Die Foraminiferen der Westphälischen Kreideformation. Sitzungsberichte der K. Akademie der Wissenschaften in Wien, Mathematisch-naturwissenschaftliche Classe, 40:147-238.

Reuss, A.E. 1862a. Paläontologische Beiträge. Sizungsberichte der Kaiserlichen Akademie der Wissenschaften in Wien, Mathematischnaturwissenschaftliche Classe (1861), 44(1):301342.

Reuss, A.E. 1862b. Entwurf einer systematischen Zusammenstellung der Foraminiferen. Sizungsberichte der Kaiserlichen Akademie der Wissenschaften in Wien, MathematischNaturwissenschaftliche Classe (1861), 44(1):355396.

Reuss, A.E. 1867. Die fossile Fauna der Steinsalzablagerung von Wieliczka in Galizien. Sitzungsberichte der K. Akademie der Wissenschaften in Wien, Mathematisch-Naturwissenschaftliche Classe, 55:17-182.

Revets, S.A. 1996a. The generic revision of the Bolivinitidae Cushman, 1927. Cushman Foundation Special Publication, 34:1-55.

Revets, S.A. 1996b. The generic revision of the Anomalinidae, Alabaminidae, Cancrisidae \& Gavelinellidae. Cushman Foundation Special Publication, 34:57113.

Reynolds, L.A. and Thunell, R.C. 1986. Seasonal production and morphologic variation of Neogloboquadrina pachyderma (Ehrenberg) in the northeast Pacific. Micropaleontology, 32:1-18.

Risso, A. 1826. Historie Naturelle des Principales Productions de l'Europe Méridionale et Particulièrment de Celles des Environs de Nice et des Alpes Maritimes, vol. 5, F.G. Levrault, Paris.

Rögl, F. 1985. Late Oligocene and Miocene planktic foraminifera of the Central Paratethys. p. 315-328. In Bolli, H.M., Saunders, J.B., and Perch-Nielsen, K. (eds.), Plankton stratigraphy: Volume 1. Cambridge University Press, Cambridge.

Rögl, F. and Bolli, H.M. 1973. Holocene to Pleistocene planktonic foraminifera of Leg 15, Site 147 (Cariaco Basin (Trench), Caribbean Sea) and their climatic interpretation. p. 553-616. In Edgar, N.T. (ed.), Initial Reports of the Deep Sea Drilling Project,15.
Rögl, F. and Hansen, H.J. 1984. Foraminifera described by Fichtel \& Moll in 1798. A revision of Testacea Microscopia. Appendix Testacea Microscopia alique minuta ex Generibus Argonauta et Nautilus. Reprint of original plates. Neue Denkschriften des Naturhistorischen Museums in Wien, 3:1-143.

Rzehak, A. 1886. Die Foraminiferenfauna der Neogenformation der Umgebung von Mahr-Ostrau: Verhandlungen des Naturforschen- den Vereins in Brünn, 24:77-126.

Said, R. 1949. Foraminifera of the northern Red Sea. Special Publications Cushman Laboratory for Foraminiferal Research 26:1-44.

Saidova, Kh.M. 1975. Bentosnye Foraminifery Tikhogo Okeana. 3 vols., Institut Okeanologii P.P. Shirshova, Akademiya Nauk SSSR, Moscow. (In Russian)

Saidova, Kh.M. 1981. O sovremennom sostoyanii sistemy nadvidovykh taksonov Kaynozoyskikh bentosnykh foraminifer. Institut Okeanologii P.P. Shirshova, Akademiya Nauk SSSR, Moscow. (In Russian)

Saito, T. 1963. Miocene Planktonic Foraminifera from Honshu, Japan. Science Reports of the Tohoku University, second series (Geology), 35:123-209.

Saito, T. 1999. Revision of Cenozoic magnetostratigraphy and the calibration of planktonic microfossil biostratigraphy of Japan against this new time scale. Journal of the Japanese Association for Petroleum Technology, 64:2-15. (In Japanese with English abstract)

Saito, T., Thompson, P.R., and Breger, D. 1981. Systematic Index of Recent and Pleistocene Planktonic Foraminifera. University of Tokyo Press, Tokyo.

Salvatorini, G. 1966. Alcuni nuova specie di foraminiferi del Miocene superiori della Toscana Marittima. Atti della Societa Toscana di Scienze Naturali Residente in Pisa Memorie Serie A, 73.

Sato, T., Saito, T., Yuguchi, S., Nakagawa, H., Kameo, K., and Takayama, T. 2002. Late Pliocene calcareous nannofossil paleobiogeography of the Pacific Ocean: evidence for glaciation at $2.75 \mathrm{Ma}$. Revista Mexicana de Ciencias Geológica, 19:175-189.

Saunders, J.B., and Müller-Merz, E. 1982. The genus Pseudononion in relationship with Nonion, Nonionella, and Nonionellina. Journal of Foraminiferal Research, 12:261-275.

Schlumberger, C. 1887. Note sur le genre Planispirina. Bulletin de la Société Zoologique de France, 12:105118.

Schlumberger, C. 1893. Monographie des Miliolidées du Golfe de Marseille. Mémoires de la Société Zoologique de France, 6:57-80.

Schröter, J.S. 1782. Neue Bemerkungen über kleine natürliche Ammonshörner. Der Naturforscher, 17:117-125.

Schröter, J.S. 1783. Einleitung in die Conchylienkenntniss nach Linné, vol. 1, J.J. Gebauer, Halle. 
Schubert, R.J. 1908. Beiträge zu einer natürlichen systematic der Foraminiferen. Neues Jahrbuch für Mineralogie, Beilage-Band, 23:232-260.

Schubert, R.J. 1910. Über Foraminiferen und einen Fischotolithen aus dem fossilen Globigerinenschlamm von Neu-Guinea. Verhandlungen der Geologischen Reichsanstalt, 1910:318-328.

Schultze, M.S. 1854. Über ded Organismus der Polythalamien (Foraminiferen), nebst Bermerkungen über die Rhizopoden im Allgemeinen. Wilhelm Engelmann, Leipzig.

Schwager, C. 1866. Fossile Foraminiferen von Kar Nikobar. Reise der Österreichischen Fregatte Novara um die Erde in den Jahren 1857, 1858, 1859 unter den Befehren des Commodore B. Von Wüllerstorf-Urbair, Geologischer Theil, 2(2: Paläontologische Mittheilungen):187-268.

Schwager, C. 1876. Saggio di una classificazione dei foraminiferi avuto riguardo alle loro famiglie naturali. Bolletino R. Comitato Geologico d'Italia, 7:475-485.

Schwager, C. 1877. Quadro del proposto sistema dei classificazione dei foraminiferi con guscio. Bolletino R. Comitato Geologico d'Italia, 8:18-27.

Schwager, C. 1878. Nota su alcuni Foraminiferi nuovi del Tubo di Stretto presso Girgenti. Bolletino R. Comitato Geologico d'Italia, 9:519-529.

Schweizer, M., Pawlowski, J., Kouwenhoven, T., and van der Zwaan, B. 2009. Molecular phylogeny of common Cibicides and related Rotaliida (Foraminifera) based on small subunit rDNA sequences. Journal of Foraminiferal Research, 39:300-315.

Schweizer, M., Pawlowski, J., Kouwenhoven, T.J., Guiard, J., and van der Zwaan, B. 2008. Molecular phylogeny of Rotaliida (Foraminifera) based on complete small subunit rDNA sequences. Marine Micropaleontology, 66:233-246.

Scott, D.B., Takayanagi, Y., Hasegawa, S., and Saito, T. 2000. Illustration and reevaluation of affinities of Neogene foraminifera described from Japan. Palaeontologia Electronica 3: 41 pp., 1.06MB; http://palaeoelectronica.org/2000_2/foram/issue2_00.htm

Seguenza, G. 1862. Prime ricerche intorno ai Rhizopodi fossili delle argille Pleistoceniche dei dintorni di Catania. Atti Accademia Gioenia Scienze Naturali, serie 2, 18:85-126.

Seguenza, G. 1880. Le formazioni Terziarie nella provincial di Reggio (Calabria). Atti Reale Accademie dei Lincei, Roma, Classe di Scienze Fisiche, Matematiche e Naturali, serie 3, 6:1-446.

Seiglie, G.A. 1965. Some observations on Recent foraminifers from Venezuela, Part I. Contributions from the Cushman Foundation for Foraminiferal Research, 16:70-73.

Seiglie, G.A. and Barker, M.B. 1987. Duquepsamminidae, a new family, and Duquepsammia, a new genus of agglutinated foraminifers. Micropaleontology, 33:263-266.
Seiglie, G.A. and Bermúdez, P.J. 1965. Monografia de la familia de foraminíferos Glabratellidae. Geos, 1965(12):15-65.

Sellier de Civrieux, J.M. 1977. Las Discorbidae del Mar Caribe, frente a Venezuela. Cuadernos Oceanográficos, Universidad de Oriente, Cumana, 6:1-44.

Shchedrina, Z.G. 1969. O nekotorykh izmeneniyakh v sisteme semeystv Astrorhizidae I Reophacidae (Foraminifera). Voprosy Mikropaleontologii, 11:157-170.

Sidebottom, H. 1910. Two new species of Cassidulina. Journal of the Quekett Microscopical Club, Second Series, 11(67):105-108.

Sigal, J. 1952. Aperçu stratigraphique sur la micropaléontologie du Crétacé. XIX Congrès Géologique International, Monographies Régionales, série 1, Algérie, 26:1-47.

Silvestri, A. 1896. Foraminiferi Pliocenici della Provincia di Siena. Parte I. Memorie della Accademia Pontificia dei Nuovi Lincei, Roma, 12:1-204.

Silvestri, A. 1902. Lageninae del mari Tirreno. Memorie della Pontificia Accademia dei Nuovi Lincei, Roma, 19:133-172.

Silvestri, A. 1903. Dimorfismo e nomenclatura d'una Spiroplecta. Altre notizie sulla struttura della Siphogenerina columellaris. Atti della Pontifiscia Accademia Romana dei Nuovi Lincei, Roma, 12:1-204.

Silvestri, A. 1904. Ricerche strutturali su alcune forme dei Trubi di Bonfornello (Palermo). Memorie della Pontificia Accademia dei Nuovi Lincei, Roma, 22:235-276.

Silvestri, A. 1923. Lo stipite deella Elissoforme e le sue affinità. Memorie della Pontificia Accademia della Scienze, Nuovi Lincei, ser. 2, 6:231-270.

Silvestri, A. 1924. Fauna Paleogenica di Vasciano presso Todi. Bollettino della Società Geologica Italiana (1923), 42:7-29.

Soldani, A. 1791. Testaceographiae ac Zoophytographiae parvae et microscopiae, vol. 1., Rossi, Senis.

Srinivasan, M.S. 1966. Descriptions of new species and notes on taxonomy of foraminifera from the upper Eocene and lower Oliogocene of New Zealand. Transactions of the Royal Society of New Zealand, Geology, 3:231-256.

Srinivasan, M.S. and Kennett, J.P. 1974. Secondary calcification of the planktonic foraminifer Neogloboquadrina pachyderma as a climatic index. Science, 186(4146):630-632.

Srinivasan, M.S. and Kennett, J.P. 1981. A review of Neogene planktonic foraminiferal biostratigraphy:applications in the Equatorial and South Pacific, p. 395-432. In Warme, J.E., Douglas, R.G., and Winterer, E.L. (eds.), The Deep Sea Drilling Project: $A$ Decade of Progress. SEPM Special Publication no. 32. Tulsa, Oklahoma.

Stache, G. 1864. Die Foraminiferen der tertiären Mergel des Whaingaroa-Hafens (Prov. Auckland), NovaraExpedition, 1857-1859, vol. 1, Geologische Theil, 2:159-304. 
Stainforth, R.M. 1952. Classification of uniserial calcareous Foraminifera. Contributions from the Cushman Foundation for Foraminiferal Research, 3:6-14.

Stewart, R.E. and Stewart, K.C. 1930. Post Miocene foraminifera from the Ventura Quadrangle, Ventura County, California. Journal of Paleontology, 4:60-72.

Tai, Y. 1954. Miocene smaller foraminifera from the Tsuyama Basin, Okayama Prefecture, Japan. Journal of Science of the Hiroshima University, Series C, 1:1-25.

Takata, H., Irizuki, T., and Ishida, K. 2006. Living benthic foraminifera from Urauchi Bay, Kamikoshiki-jima Island, Kagoshima Prefecture, southern Japan. LEGUNA 13: 99-107. (In Japanese with English abstract)

Takayanagi, Y. 1953. New genus and species of foraminifera found in the Tonohama Group, Kochi Prefecture, Shikoku, Japan. Short Papers from the Institute of Geology and Paleontology, Tohoku University, 5:25-36.

Takayanagi, Y. and Hasegawa, S. 1987. Checklist and Bibliography of Post-Paleozoic Foraminifera Established by Japanese Workers, 1890-1986. Institute of Geology and Paleontology, Tohoku University, Sendai, Japan.

Takayanagi, Y. and Saito, T. 1962. Planktonic foraminifera from the Nobori Formation, Shikoku, Japan. Science Reports of the Tohoku University, Sendai, second series (Geology), Special Volume 5 (Kon'no Memorial Volume):67-106.

Thalmann, H.E. 1933. Zwei neue Vertreter der Foraminiferen-Guttung Rotalia Lamarck, 1804: R. cubana nom. nov. und $R$. trispinosa nom. nov. Eclogae geologicae Helvetiae, 26:248-251.

Thalmann, H.E. 1937. Mitteilungen über Foraminiferen III. Weitere Nomina Mutata in Brady's Werk über die Foraminiferen der 'Challenger'-Expedition (1884). Eclogae geologicae Helvetiae, 30:340-342.

Thalmann, H.E. 1939. Bibliography and index to new genera, species and varieties of foraminifera for the year 1936. Journal of Paleontology, 13:425-465.

Thalmann, H.E. 1952. Bibliography and index to new genera, species and varieties of foraminifera for the year 1951. Journal of Paleontology, 26:953-992.

Todd, R. 1957. Smaller Foraminifera, p. 265-320. In Geology of Saipan Mariana Islands, Part 3. Paleontology, United States Geological Survey, Professional Paper, 280-H.

Todd, R. 1965. The Foraminifera of the Tropical Pacific Collections of the "Albatross," 1899-1900. Part 4. Rotaliform families and Planktonic families. United States National Museum Bulletin, 161.

Toulmin, L.D. 1941. Eocene smaller foraminifera from the Salt Mountain Limestone of Alabama. Journal of Paleontology, 15:567-611.
Trauth, F. 1918. Das Eozänvorkommen bei Radstadt im Pongau und seine Beziehungen zu den gleichalterigen Ablagerungen bei Kirchberg am Wechsel und Wimpassing am Leithagebirge. Denkschriften der Kaiserlichen Akademie der Wissenschaften Wien, Mathematisch-naturwissenschaftliche Classe, 95:171-278.

Tsuburaya, H. and Sato, T. 1985. Kisoshisui "Miyakojimaoki". Journal of the Japanese Association for Petroleum Technology, 50:25-33. (In Japanese)

Uchio, T. 1951. New species and genus of the foraminifera of the Cenozoic formations in the middle part of the Boso Peninsula, Chiba-ken, Japan. Transaction and Proceedings of the Palaeontological Society of Japan, New Series, 2:33-42.

Uchio, T. 1952. Geology of natural gas in the western part of Mobara-machi, Chiba Prefecture. Journal of the Japanese Association for Petroleum Technologists, 17:22-37 (In Japanese with abstract and description of new species in English)

Uchio, T. 1953. On some foraminiferal genera in Japan. Japanese Journal of Geology and Geography, 23:151-162.

Uchio, T. 1960. Ecology of living benthonic foraminifera from the San Diego, California area. Cushman Foundation for Foraminiferal Research, Special Publication, 5:1-72.

Uchio, T. 1962. Influence of the River Shinano on foraminifera and sediment grain size distributions. Publications of the Seto Marine Biological Laboratory, 10:363-392.

Ujiié, H. 1966. Shell structure of Japanese smaller foraminifera, Part 2. Pararotalia nipponica (Asano, 1936). Transaction and Proceedings of the Japanese Palaeontological Society of Japan, New Series, 61:191200.

Ujiié, H. 1985. A standard late Cenozoic microbiostratigraphy in southern Okinawa-jima, Japan. Part 2. Details on the occurrence of planktonic foraminifera with some taxonomic annotations. Bulletin of the National Science Museum, Series C (Geology and Paleontology), 11:103-136.

Ujiié, H. 1990. Bathyal benthic foraminifera in a piston core from east off the Miyako Islands, Ryukyu Island Arc. Bulletin of the College of Science, University of the Ryukyus, 49:1-60.

Ujiié, H. 1994. Early Pleistocene birth of the Okinawa Trough and Ryukyu Island Arc at the northwestern margin of the Pacific: evidence from Late Cenozoic planktonic foraminiferal zonation. Palaeogeography, Palaeoclimatology, Palaeoecology, 108:457-474.

Ujiié, H. 1995. Benthic foraminifera common in the bathyal surface sediments of the Ryukyu Island Arc region, Northwest Pacific. Bulletin of the College of Science, University of the Ryukyus, 60:51-111.

Ujiié, H. 2003. A 370-ka paleoceanographic record from the Hess Rise, central North Pacific Ocean, and an indistinct 'Kuroshio Extension'. Marine Micropaleontology, 49:21-47. 
Ujiié, H. and Hatta, A. 1995. Quantitative analyses of benthic foraminiferal assemblages from southern Ryukyu Island Arc, subtropical northwestern Pacific Ocean. Journal of Foraminiferal Research, 25:334349.

Ujiié, H. and Kusukawa, T. 1969. Analysis of foraminiferal assemblages from Miyako and Yamada Bays, Northeastern Japan. Bulletin of the National Science Museum, Series C (Geology and Paleontology), 12:735-772.

Ujiié, H. and Ōki, K. 1974. Uppermost Miocene-Lower Pleistocene planktonic foraminifera from the Shimajiri Group of Miyako-jima, Ryukyu Islands. Memoirs of National Science Museum, Tokyo, 7:31-58.

Ujiié, H., Ichikura, M., and Kurihara, K. 1983. Quaternary benthonic foraminiferal changes observed in the Sea of Japan piston cores. Bulletin of the National Science Museum, Series C (Geology and Paleontology), 9:41-78.

Van den Broeck, E., 1876. Étude sur les foraminifères de la Barbade (Antilles). Annales Société Belge de Microscopie, Bruxelles 2 (1875-1876):55-152.

Van Marle, L.J. 1988. Bathymetric distribution of benthic foraminifera on the Australian-Irian Jaya continental margin, eastern Indonesia. Marine Micropaleontology, 13:97-152.

Vella, P. 1957. Studies in New Zealand foraminifera: Part I-Foraminifera from Cook Strait. Part II-Upper Miocene to Recent species of the genus Notorotalia. New Zealand Geological Survey Paleontological Bulletin, 28:1-64.

Vella. P. 1962. Late Tertiary nonionic foraminifera from Wairarapa, New Zealand. Micropaleontology, 7:467483.

Vella, P. 1963. Some foraminifera from the upper Miocene and Pliocene of Wairarapa, New Zealand. Transactions of the Royal Society of New Zealand, Geology, 2(1):1-14.

Voloshinova, N.A. 1958. O novoy sistematike Nonionid. Trudy Vsesoyuznogo Neftyanogo Nauchno-issledovatel'skogo Geologo-razvedochnogo Instituta (VNIGRI), 115:117-191 (Mikrofauna SSSSR Sbrnik 9). (In Russian)

Voloshinova, N.A. 1960. Uspekhi mikropaleontologii v dele izucheniya vnutrennego stroeniya foraminifer. $p$. 48-87. In Trudy Pervogo Seminara po Milrofaune. Vsesoyuznyy Neftyanoy Nauchno-issledovatel'skii Geologo-razvedochnyy Institut (VNIGRI), Leningrad. (In Russian)

Voloshinova, N.A. and Dain, L.G. 1952. Iskopaemye Foraminifery SSSR. Nonionidy, Kassidulinidy I Khilostomellidy. Trudy, Vsesoyuznogo Neftyanogo Nauchno-issledovatel'skogo Geologo-razvedochnogo Instituta (VNIGRI), new series, 63:1-151. (In Russian)

von Eichwald, C.E. 1830. Zoologia specialis vol. 2, D.E. Eichwaldus, Vilnae. von Fichtel, L., and von Moll, J.P.C. 1798. Testacea microscopica, aliaque minuta ex generibus Argonauta et Nautilus, ad naturam picta et descripta (Microscopische und andere klein Schalthiere aus den geschlechtern Argonaute und Schiffer). Camesina, Vienna.

Walters, R. 1965. The Globorotalia zealandica and G. miozea lineages. New Zealand Journal of Geology and Geophysics, 8:109-127.

Waller, H.O. and Polski, W. 1959. Planktonic foraminifera of the Asiatic shelf. Contributions from the Cushman Foundation for Foraminiferal Research, 10:123-126.

Wang, P. and Lutze, G.F. 1986. Inflated later chambers: ontogenetic changes of some recent hyaline benthic foraminifera. Journal of Foraminiferal Research, 16:48-62.

Wang, P., Zhang, J., Zhao, Q., Min, Q., Bian, Y., Zheng, L., Cheng, X., and Chen, R. 1988. Foraminifera and Ostracoda in bottom sediments of the East China Sea. The Ocean Press, Peking. (In Chinese with English summary)

Wedekind, P.R. 1937. Einführung in die Grundlagen der historischen Geologie. Band II. Mikrobiostratigraphie die Korallen- und Foraminiferenzeit. Ferdinand Enke, Stuttgart.

Weinholz, P. and Lutze, G.F. 1989. The Stilostomella extinction. p. 113-117. In Ruddiman, W. F. et al. (eds.), Proceedings of the Ocean Drilling Program Scientific Results, 108, College Station, TX (Ocean Drilling Program).

Wiesner, H. 1920. Zur Systematik der Miliolideen. Zoologisches Anzeiger, 51:13-20.

Wiesner, H. 1931. Die Foraminiferen der deutschen Südpolar Expedition 1901-1903. Deutsche SüdpolarExpedition, 20 (Zoologie, 12):53-165.

Williamson, W.C. 1848. On the Recent British species of the genus Lagena. Annals and Magazine of Natural History, Second Series, 1:1-20.

Williamson, W.C. 1858. On the Recent Foraminifera of Great Britain. The Ray Society, London.

Wright, J. 1880. The post-Tertiary Foraminifera of the North East of Ireland. Proceedings Belfast Naturalists' Field Club, 1879-1880, Appendix:149-163.

Xu, X. and Ujiié, H. 1994. Bathyal benthic foraminiferal changes during the past 210,000 years: evidence from piston cores taken from seas south of Ishigaki Island, southern Ryukyu Island Arc. Transactions and Proceedings of Palaeontological Society of Japan, New Series, 175:497-520.

Xu, J., Wang, P., Huang, B., Li, Q., and Jian, Z. 2005. Response of planktonic foraminifera to glacial cycles: Mid-Pleistocene change in the southern South China Sea. Marine Micropaleontology, 5:89-105.

Yamazato, K. 1960. On the limestone of Miyako Island. Bulletin of Arts and Science Division, University of the Ryukyus. Mathematics and Sciences, 4:88-92. 
Yazaki, K. and Oyama, K. 1979. Geology of the Miyakojima-Hokubu district. Quadrangle Series, Scale 1:50,000, Miyako-jima (19) No. 3, Geological Survey of Japan. (In Japanese with English abstract)

Yazaki, K. and Oyama, K. 1980. Geology of the Miyakojima district. Quadrangle Series, Scale 1:50,000, Miyako-jima (19), No. 4, Geological Survey of Japan. (In Japanese with English abstract)
Zheng, Shou-Yi, 1979. The Recent foraminifera of the Xisha Islands, Guangdong Province, China. II. Studia Marina Sinica, 15:101-232.

Zheng, Shou-Yi, 1988. The agglutinated and porcellaneous Foraminifera of the East China Sea. China Ocean Press, Beijing. 